\begin{tabular}{|l|c|}
\hline JAN 1998 & Page 1 of $\frac{1}{1}$ \\
Qta. .37 & i. edr 622746 \\
\hline
\end{tabular}

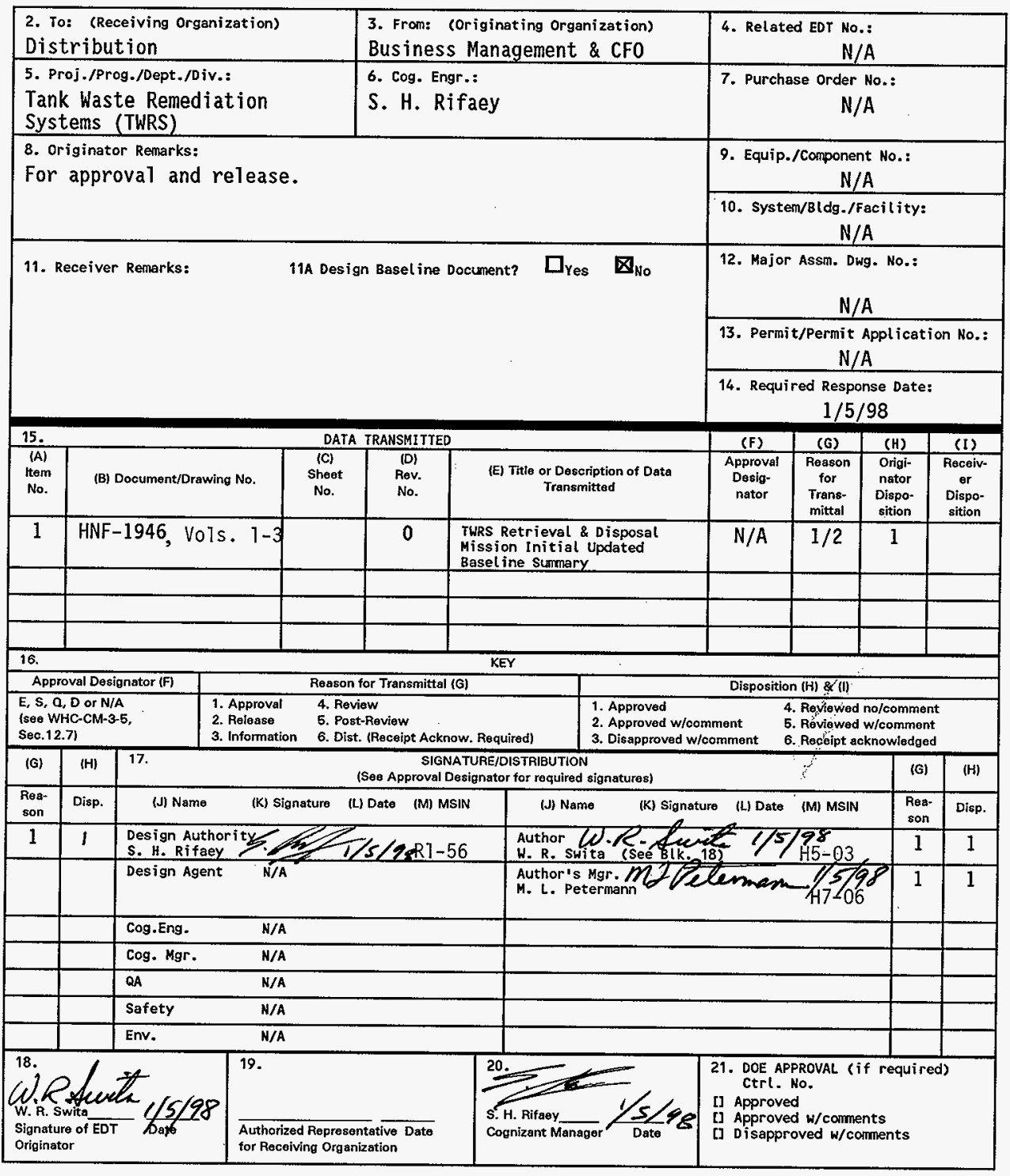




\title{
Tank Waste Remediation System Retrieval and Disposal Mission Initial Updated Baseline Summary
}

\author{
W.R. Swita \\ Lockheed Martin Hanford Company, Richland, WA 99352 \\ U.S. Department of Energy Contract DE-AC06-96RL13200
}

$\begin{array}{ll}\text { EDT/ECN: } 622746 & \text { UC: } 2030 \\ \text { Org Code: } 72220 & \text { Charge Code: D215P } \\ \text { B\&R Code: EW3130010 } & \text { Total Pages: }-793 \text { Pa }\end{array}$

Key Words: TWRS, Retrieval and Disposal Mission, Baseline Summary

Abstract: This document provides a summary of the proposed Tank Waste Remediation System Retrieval and Disposal Mission Initial Updated Baseline (scope, scheduTe, and cost) developed to demonstrate the Tank Waste Remediation System contractor's Readiness-to-Proceed in support of the Phase 1B mission.

TRADEMARK DISCLAIMER. Reference herein to any specific commercial product, process, or service by trade name, trademark, manufacturer, or otherwise, does not necessarily constitute or imply its endorsement, recommendation, or favoring by the United States Government or any agency thereof or its contractors or subcontractors.

Printed in the United States of America. To obtain copies of this document, contact: Document Control Services, P.0. Box 950, Mailstop H6-08, Richland WA 99352, Phone (509) 372-2420; Fax (509) 376-4989.
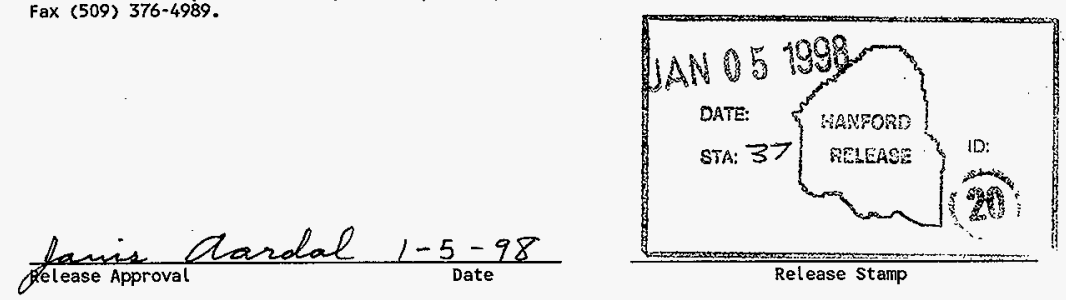


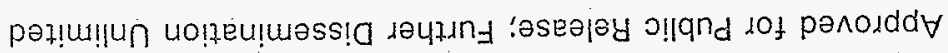

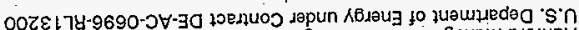

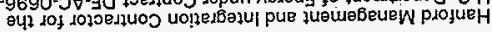
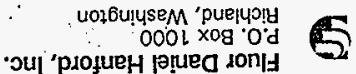

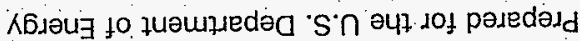
uofbuịse $M$ 'pue|yจ!y uonesodioj plojueH u!

8661 Nuenuer

paysugrid әzeg

\author{
$3 \exists \perp O \forall W$ \\ $\| ! B N, O \cdot W$
}

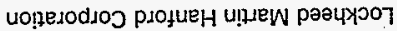

Sima7 $\mathrm{d} \cdot \mathrm{W}$

ef!MS $y \cdot M$

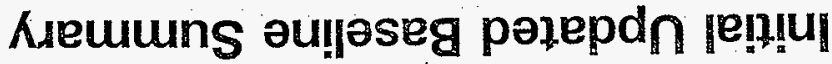 uolss!n jesods!a pue jenolnoy

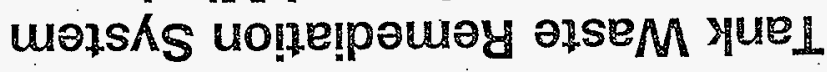


HNF-1946 Rev. 0

Document Title: Tank Waste Remediation System Retrieval and Disposal Mission Initial Updated Baseline Summary

Approved by:

\section{H. L. Boston, Vice President}

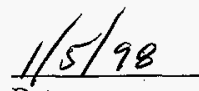

Tank Waste Retrieval

Lockheed Martin Hanford Corporation

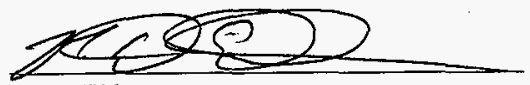

M. D. Ebben

Date

Business Management and $\mathrm{CFO}$

Lockheed Martin Hanford Corporation

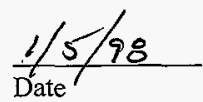


HNF-1946 Rev. 0

This page intentionally left blank. 
HNF-1946 Rev. 0

\section{EXECUTIVE SUMMARY}

This document provides a summary of the proposed Tank Waste Remediation System. Retrieval and Disposal Mission Initial Updated Baseline (scope, schedule, and cost) developed to demonstrate the Tank Waste Remediation System contractor's Readiness-to-Proceed in support of the Phase $1 B$ mission.

The document is comprised of the following sections:

- An introduction, including a summary of the document and a description of the Integrated Baseline planning process used to produce the scope, schedule, and cost exhibits

- $\quad$ Summary scope descriptions and exhibits, including Level I Logics which are key documents for scope definition

- Summary schedule descriptions and exhibits, including an updated Project Master Baseline Schedule depicting the Level 1 Logic activities

- Summary cost descriptions and exhibits, including a summary spreadsheet and cost-loading report depicting costs resulting from the logic-driven, resourceloaded schedule 
HNF-1946 Rev. 0

- Summary program-level description of changes from the Fiscal Year 1998 Tank

Waste Remediation System Multi-Year Work Plan ${ }^{1}$ to the proposed Readiness-toProceed Initial Updated Baseline.

This proposed Baseline has been integrated with the Mission Definition and Direction, Management Plans and Procedures, and the Technical Baseline. The scope, schedules, and associated costs demonstrate that the proposed baseline fully supports the private contractors' facilities for Phase $1 B$ waste treatment. Necessary support activities include the following.

- $\quad$ Provide Waste Feed Delivery.

- $\quad$ Provide interim storage of immobilized high-level waste products.

- $\quad$ Provide disposal of immobilized low-activity waste products.

- Provide disposal of byproduct wastes generated by the private contractors.

- Provide the infrastructure to support construction and operations of the private. contractors' facilities.

The data contained in this proposed Baseline have been incorporated in the Tank Waste Remediation System Retrieval and Disposal Mission Phase 1 Financial Analysis ${ }^{2}$ and will serve

${ }^{1}$ Lenseigne, D. L., 1997, Tank Waste Remediation System Fiscal Year 1998 Multi-Year Work Plan WBS 1.1, HNF-SP-1230, Rev. 0, prepared by Lockheed Martin Hanford Corporation for Fluor Daniel Hanford, Inc., Richland, Washington.

${ }^{2}$ Wells, M. W., 1998, Tank Waste Remediation System Retrieval and Disposal Mission Phase 1 Financial Analysis, HNF-2017, Rev. 0, DRAFT, prepared by Lockheed Martin Hanford Corporation for Fluor Daniel Hanford, Inc., Richland, Washington. 
HNF-1946 Rev. 0

as the basis for a subsequent formal change to the Tank Waste Remediation System Fiscal Year 1998 Multi-Year Work Plan Baseline.

vii 
HNF-1946 Rev. 0

This page intentionally left blank.

viii 
HNF-1946 Rev. 0

\section{CONTENTS}

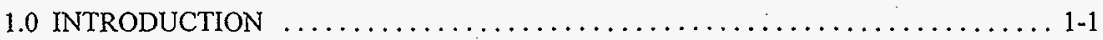

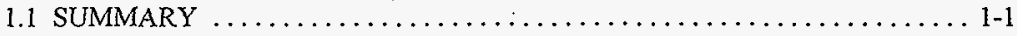

1.2 BASELINE PLANNING PROCESS (SCOPE, SCHEDULE, AND COST) ... 1-3

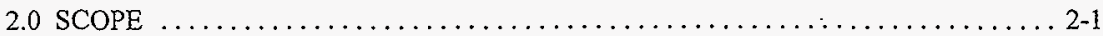

2.1 TANK WASTE REMEDIATION SYSTEM PROGRAM LOGIC ........ 2-1

2.2 TWRS RETRIEVAL AND DISPOSAL WORK BREAKDOWN

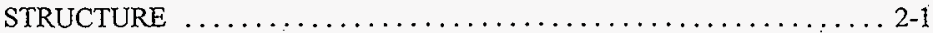

2.3 FEED DELIVERY, STORAGE AND DISPOSAL MISSION SUMMARY $\ldots .2$ 2-1

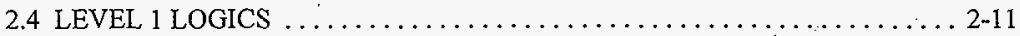

2.5 CROSSWALK FROM THE LEVEL 1 LOGIC TO THE WORK

BREAKDOWN STRUCTURE ...................... 2-11

2.6 TWRS SCOPE/COST NOT INCLUDED IN THE INITIAL

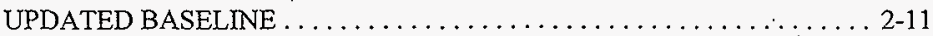

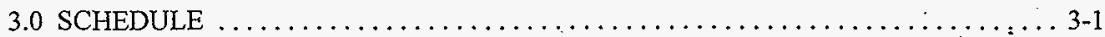

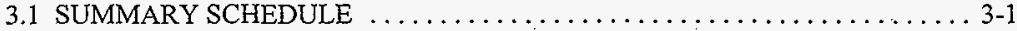

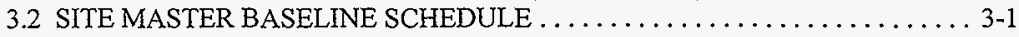

3.3 PROJECT MASTER BASELINE SCHEDULE $\ldots \ldots \ldots \ldots \ldots \ldots \ldots \ldots . .1$

3.4 MLESTONE COMPARISON $\ldots \ldots \ldots \ldots \ldots \ldots \ldots \ldots \ldots \ldots \ldots \ldots, 3-1$

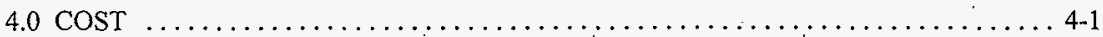

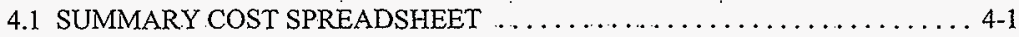

4.2 DESCRIPTION OF THE TYPES OF COSTS $\ldots \ldots \ldots \ldots \ldots \ldots \ldots \ldots \ldots, 4-1$

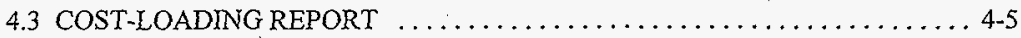

5.0 SIGNIFICANT PROGRAM-LEVEL CHANGES (SCOPE, SCHEDULE, COST) ... . . 5-1

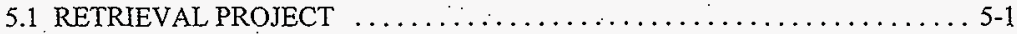

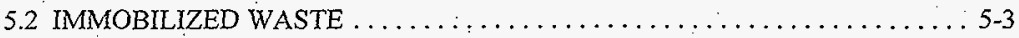

5.3 PRIVATIZATION INFRASTRUCTURE $\ldots \ldots \ldots \ldots \ldots \ldots \ldots \ldots \ldots .4 .4$

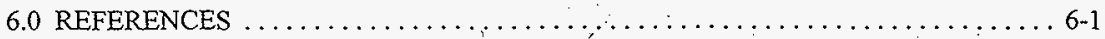

APPENDICES

A. LEVEL 1 LOGICS $\ldots \ldots \ldots \ldots \ldots \ldots \ldots \ldots \ldots \ldots \ldots \ldots \ldots \ldots \ldots \ldots \ldots \ldots \ldots \ldots \ldots$

B GUIDANCE AND REQUIREMENTS TO DELIVERABLES CROSSWALK TWRS RETRIEVAL AND DISPOSAL MISSION INITIAL UPDATED

BASELINE SUMMARY $\ldots \ldots \ldots \ldots \ldots \ldots \ldots \ldots \ldots \ldots \ldots \ldots \ldots \ldots, \ldots \ldots \ldots$ 
HNF-1946 Rev. 0

C GUIDANCE AND REQUUREMENTS TO DELIVERABLES

CROSSWALK - LEVEL 0 AND LEVEL 1 LOGICS $\ldots \ldots \ldots \ldots \ldots \ldots \ldots$ C-i

VOLUME 2 LEVEL 1 LOGICS

LOW-ACTIVITY WASTE FEED LEVEL 1 LOGICS

Figure A-1 LAW 1st Feed Batches, Tank 241-AN-105

Figure A-2 LAW 2nd Feed Batches, Tank 241-AN-104

Figure A-3 LAW 3rd Feed Batches, Tank 241-AW-101

Figure A-4 LAW 4th Feed Batches, Tank 241-AN-103

Figure A-5 LAW 5th Feed Batches, Tanks 241-AP-101 \& 241-AW-104

Figure A-6 LAW 6th Feed Batches, Tank 241-AY-101

Figure A-7 LAW 7th \& 8th Feed Batches, Tank 241-AN-107

Figure A-8 LAW 9th Feed Batches, Tank 241-AN-102

Figure A-9 LAW 10th Feed Batches, Tank 241-AN-106

VOLUME 3 LEVEL 1 LOGICS

\section{LOW-ACTIVITY WASTE FEED LEVEL 1 LOGICS}

Figure A-10 LAW 11th Feed Batches, Tank 241-SY-101

Figure A-11 LAW 12th Feed Batches, Tank 241-SY-103

HIGH-LEVEL WASTE FEED LEVEL I LOGICS

Figure A-12 HLW 1st \& 2nd Feed Batches, First Tank 241-AZ-101

Figure A-13 HLW 3rd \& 4th Feed Batches, First Tank 241-AZ-102

Figure A-14 HLW 5th - 9th Feed Batches, 241-AY-102

Figure A-15 HLW 10th - 12th Feed Batches, Tank 241-C-104

IMMOBILIZED LOW-ACTIVITY WASTE LEVEL 1 LOGICS

Figure A-16 Immobilized Waste ILAW

IMMOBILIZED HIGH-LEVEL WASTE LEVEL 1 LOGICS

Figure A-17 Immobilized Waste IHLW

INFRASTRUCTURE PHASE 1 LEVEL 1 LOGICS

Figure A-18 Phase 1 Privatization Support 
HNF-1946 Rev. 0

\section{LIST OF FIGURES}

1. Readiness-to-Proceed Document Hierarchy.

2. Tank Waste Remediation System Retrieval and Disposal Mission Initial Updated Baseline Summary Documents. $1-4$

3. Tank Waste Remediation System Program Logic.

4. Tank Waste Retrieval and Disposal Work Breakdown Structure. $\ldots \ldots \ldots \ldots \ldots \ldots .2-5$

5. Feed Delivery, Storage and Disposal Mission Summary.

6. Summary Schedule.

7. Site Master Baseline Schedule

8. Project Master Baseline Schedule.

\section{LIST OF TABLES}

1. Crosswalk from the Level 1 Logic to the Work Breakdown Structure Level 6 $2-13$

2. Milestone Comparison.

3. Summary Cost Spreadsheet. 4-3

4. Cost-Loading Report. 4-7 
HNF-1946 Rev. 0

\section{LIST OF TERMS}

CEIS

DST

FY

HLW

LAW

MYWP

PHMC

$\mathrm{RL}$

RTP

SST

Tri-Party Agreement

TBR

TWRS

WBS cost estimating input sheet

double-shell tank

fiscal year

high-level waste

low-activity waste

Multi-Year Work Plan

Project Hanford Management Contract

U.S. Department of Energy, Richland Operations Office

Readiness-to-Proceed

single-shell tank

Hanford Federal Facility Agreement and Consent Order

Technical Basis Review

Tank Waste Remediation System

Work Breakdown Structure 
HNF-1946 Rev. 0

\section{TANK WASTE REMEDIATION SYSTEM RETRIEVAL AND DISPOSAL MISSION INITIAL UPDATED BASELINE SUMMARY}

\subsection{INTRODUCTION}

\subsection{SUMMARY}

This document provides a summary of the Tank Waste Remediation System (TWRS) Retrieval and Disposal Mission Initial Updated Baseline (scope, schedule, and cost), developed to demonstrate Readiness-to-Proceed (RTP) in support of the TWRS Phase $1 \mathrm{~B}$ mission. This Updated Baseline is the proposed TWRS plan to execute and measure the mission work scope.

This document and other supporting data demonstrate that the TWRS Project Hanford Management Contract (PHMC) team is prepared to fully support Phase $1 \mathrm{~B}$ by executing the following scope, schedule, and cost baseline activities.

- Deliver the specified initial low-activity waste (LAW) and high-level waste (HLW) feed batches in a safe manner to support private contractors' operations in June 2002.

- Deliver specified subsequent LAW and HLW feed batches during Phase $1 \mathrm{~B}$ in a consistent, safe, and reliable manner.

- $\quad$ Provide for the interim storage of immobilized HLW products and the disposal of immobilized LAW products generated by the private contractors.

- Provide for disposal of byproduct wastes generated by the private contractors and returned to the TWRS.

- Provide the infrastructure to support construction and operations of the private contractors' facilities.

As shown on Figure 1, Readiness-to-Proceed Document Hierarchy, and as discussed in the Tank Waste Remediation System Retrieval and Disposal Mission Readiness-to Proceed Memorandum (Boston and Jordan 1998) and the Management Assessment of Tank Waste Remediation System Contractor Readiness-to-Proceed with Phase $1 B$ Privatization (Payne et al. 1998), this Initial Updated Baseline (HNF-1946) or the Programmatic Baseline is a key element of the Integrated Baseline. The Integrated Baseline also includes the Technical 
HNF-1946 Rev. 0

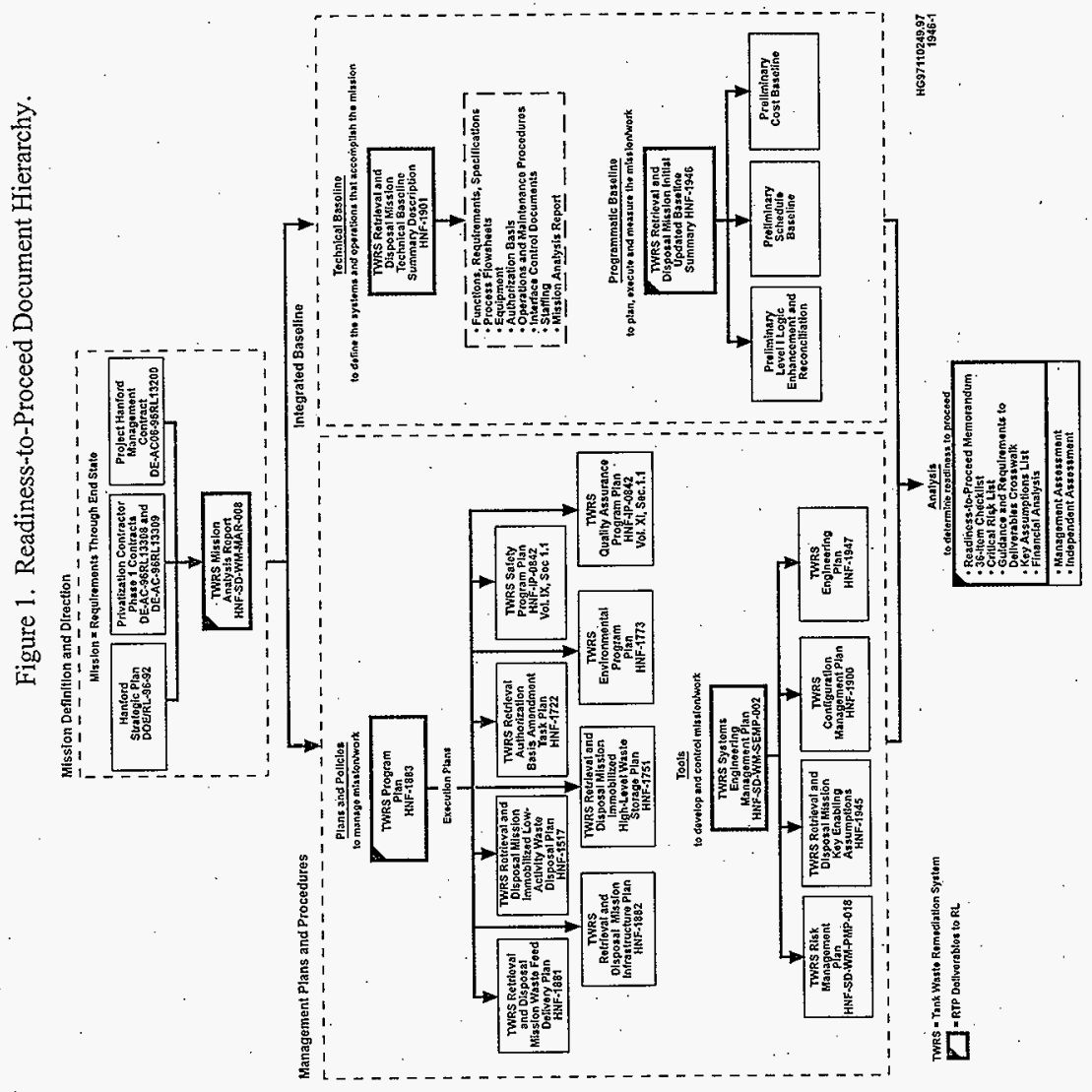


Baseline as documented in HNF-1901, Tank Waste Remediation System Retrieval and Disposal Mission Technical Baseline Summary Description (Treat et al. 1998).

The Programmatic Baseline is consistent with and reflects the scope of work defined in HNF-1901 (Treat et al. 1998). Similarly, this Programmatic Baseline is consistent with and reflects the scope, schedule, and cost necessary to execute the Management Plans and Procedures for the mission/work including the Plans and Policies documented in the Tank Waste Remediation System Program Plan (Freeman 1998) and sub-tier plans as well as the Tools to develop and control the mission/work as documented in the Tank Waste Remediation System Systems Engineering Management Plan (Peck 1998). The Programmatic Baseline is similarly consistent with the top-level Mission Definition and Direction documented in the Tank Waste Remediation System Mission Analysis Report (Acree 1998).

Figure 2 is a graphical depiction of the summary-level exhibits contained in this document. This document includes a short narrative that describes each of the exhibits for scope, schedule, and cost. Additional supporting documentation is available, as requested.

This Initial Updated Baseline represents a more thorough analysis of the scope, schedule, and cost than is contained in the current Tank Waste Remediation System Fiscal Year 1998 Multi-Year Work Plan (MYWP) (Lenseigne 1997) Baseline and will serve as the basis for a change to the TWRS Baseline. A discussion of the systematic and controlled planning process used during RTP to develop the Initial Updated Baseline follows.

\subsection{BASELINE PLANNING PROCESS (SCOPE, SCHEDULE, AND COST)}

To conduct the RTP planning process, senior level multi-functional planning teams were formed with technically knowledgeable representatives from Operations; Characterization; . Retrieval; Projects; Nuclear Safety; Environmental, Safety, and Health and Quality Assurance; Engineering/Maintenance; U.S. Department of Energy, Richland Operations Office (RL)/Waste Integration Team; Business Management (scheduling and cost estimating); and the Chief Financial Officer. Team leads were assigned to the planning effort commensurate with the type of work being addressed. Use of these multi-functional planning teams ensures that interfaces are identified among performing organizations for each activity and series of activities and that work is integrated between programs, projects, and operations. Using technical product descriptions prepared by the RTP team and integrated planning teams, the Initial Updated Baseline, depicted in Figures 1 and 2, was prepared.

The work scope for the Initial Updated Baseline is defined by Level 1 Logics prepared during RTP for LAW and HLW feed delivery, Privatization Infrastructure, and Immobilized Waste. The logics are included in Appendix A. These logics portray the required activities to complete the Phase 1B TWRS Retrieval and Disposal mission. Scope driven by logic development ensures consistency with the technical requirements and mission and validates the 
Figure 2. Tank Waste Remediation System Retrieval and Disposal Mission Initial Updated Baseline Summary Documents.

\section{Summary Documents}

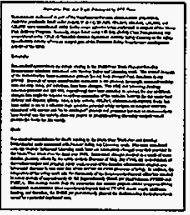

Introduction

Description of the

updated baseline and

baseline development process
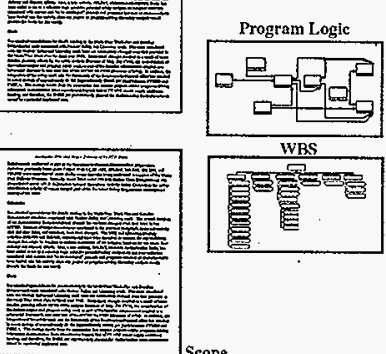

Scope

Description of

scope contert

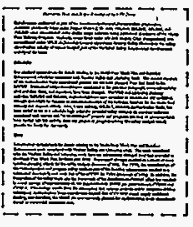

Summary Schedule

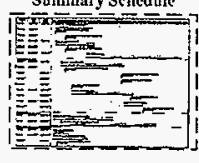

Feed Delivery, Storag and Dispisal
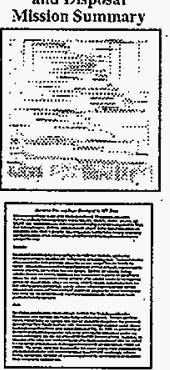

TYWRS scope/cost not included in the initial updated baseline

Schedule

Description of schedule content

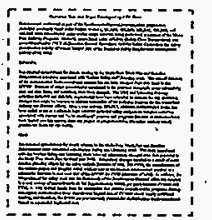

Summary Cost Spreadshcet

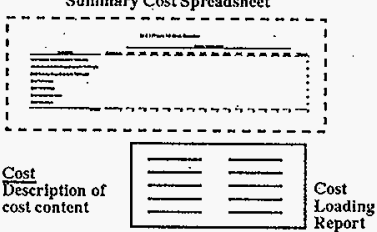

Level 1 Logics

Waste Feed Delivery

(LAbV and HLWV)

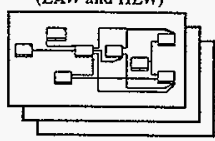

Yunmobilized Waste
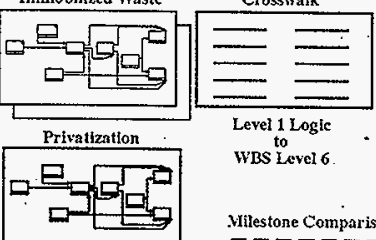

Site Master Baseline Schedule

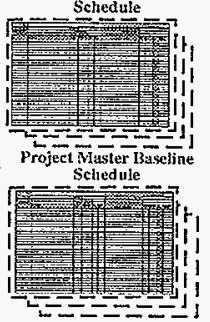

Milestone Comparison

厂

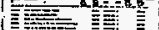

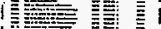

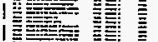

I

| 产量证

春㘳
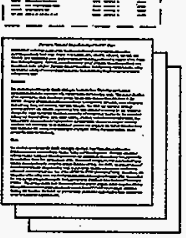

Program level

deveription of

significant

changes

- Scope

- Schedule

- Cost

Description

of the types of cost
$\underline{\mathrm{KEY}}$

Scope
TWRS = Tank Waste Kemediation System

WhS = Work Breakdown Siructure.

LAW $=$ Low-Activity Wast
HitW $=$ Hligh-Level Liaste 
HNF-1946 Rev. 0

need for the work. These Level 1 Logics replace the preliminary Feed Delivery Mid-Level Logics developed in fiscal year (FY) 1997 for the current MYWP. Each activity on the Level 1 Logic correlates to Level 7 of the TWRS Retrieval and Disposal work breakdown structure (WBS). Upon completion and validation of the Level 1 Logics by the planning team, a Technical Basis Review (TBR) sheet was prepared to fully document and define the activity. The TBR sheets include the following information:

- Header information for activity identification, title, responsible organization, responsible manager, technical content, and duration

- Technical basis

- Reference documents and reports

- Enabling assumptions

- Functional requirements and deliverables

- Connection with other activities and/or organizations .

- Trade studies

- Decisions made/required

- $\quad$ Risk issues

- Plan to resolve risk

- Contacts

- Further action recommended.

These TBR sheets were written in accordance with a desk instruction for their preparation and acceptance.

Using the scope and activity definition provided by the Level 1 Logic and TBR sheet, the planning teams prepared draft TBR packages consisting of TBR sheets, Level 2 task or subactivity logic networks, Level 2 Cost Estimating Input Sheets (CEIS), and cost loading reports generated by the Primavera Systems, Inc. scheduling software. This level of detail was necessary to adequately define and document the basis for the scope, schedule, and resource estimate at an executable task level. The Level 2 subactivity logics provide titles for the tasks, predecessor and successor activities, durations, and logic ties. The Level 2 CEIS defines the 
scope, resources, and basis of estimate for each task. The following data are documented on each CEIS:

- Header information including task title, TBR number, activity owner, prepared by, date prepared, date updated, schedule activity identification number, financial data system work package/task package number, and duration

- Resource description

- Job category

- Organization code

- Cost element

- $\quad$ Fund type

- Note reference

- Total amount (of resource units)

- Units duration/tag

- Sub-task number

- Sub-task description

- Scope description

- Estimating assumptions/exclusions/risks

- Basis of estimate

- Estimate stage and method

- Reference documents

- Notes.

The CEIS's were prepared by the planning teams with the assistance of professional schedulers and cost estimators using detailed desk instructions and guidelines. These completed estimates are activity based and represent a well-documented, traceable scope and basis for estimate at the executable task level commensurate with the stage of the work and the level of scope definition available. For Immobilized Waste, updated Activity Planning Forms and 
Estimating Work Sheets were prepared instead of the CEIS to document the required information.

Utilizing the Level 1 Logics and data from the TBR packages, a detailed integrated schedule was developed in the Primavera Systems, Inc. scheduling software. This schedule is a task-oriented, logically driven, resource-loaded critical path schedule that was summarized to produce the Site Master Baseline Schedule (refer to Section 3.2, Figure 7) and the Project Master Baseline Schedule (refer to Section 3.3, Figure 8). This schedule is traceable to the logic, WBS, activity owner (performing organization), and CEIS. The schedule was reviewed and revised to refine logic, float, and critical path. Resources from the CEIS were loaded and priced in the Primavera Systems, Inc. scheduling software to produce the cost profiles for work defined in the Level 1 Logics. Fiscal year 1998 Fluor Daniel Hanford, Inc. approved labor rate tables were used in the Primavera Systems Inc. scheduling software for pricing: The final TBR packages (Rev. 0 ) were prepared from the approved schedule consisting of and supported by the TBR sheets, subactivity logics, Primavera cost loading reports, and CEIS's. These packages were signed by the activity owners and the TWRS Retrieval and Disposal Program and placed under configuration control.

In addition to the schedules and costs developed from the Level 1 Logics and TBR : packages, additional TWRS scope and costs have been included in the Initial Updated Baseline. These costs are summarized in Section 4.0, Cost. Additional detail and analysis are contained in the Financial Analysis (Wells 1998). Data developed in the TBR packages provided bottoms-up enabling assumptions and risk management information used in the Financial Analysis to assess risk. 
HNF-1946 Rev. 0

This page intentionally left blank. 
HNF-1946 Rev. 0

\subsection{SCOPE}

Scope has been defined by specific products developed/updated during the RTP development phase. These products are discussed in the following sections and are included as figures or tables in this section.

\section{- 2.1 TANK WASTE REMEDIATION SYSTEM PROGRAM LOGIC}

The TWRS Program (Level 0) Logic (Figure 3) is the top-level TWRS logic used to identify the structure of activities necessary to achieve the entire integrated TWRS mission (life cycle). The TWRS Program Logic is the top-level basis for the RTP planning and is traceable to the TWRS Retrieval and Disposal Mission Level 1 Logics. The Program Logic has been annotated to identify the major program activities for Safe Storage, Phase 1, Phase 2, and Closure.

\subsection{TWRS RETRIEVAL AND DISPOSAI WORK BREAKDOWN STRUCTURE}

The TWRS Retrieval and Disposal WBS (Figure 4) depicts all TWRS Retrieval and Disposal mission work scope to WBS Level 6 . It has been shaded to differentiate the WBS elements applicable to Phase 1 and Phase 2. The Waste Feed Delivery Level 6 WBS elements were revised as a result of RTP planning to reflect the following: (1) authorized line item project work broken out by fund type and phase; (2) the addition of Project W-314 Phase 1; and (3) additional elements to segregate Retrieval Operations into Single-Shell Tanks, Double-Shell Tanks, Characterization, and Safety Basis. This WBS is used as a framework for scope definition, scheduling, estimating, and managing the work.

\subsection{FEED DELIVERY, STORAGE AND DISPOSAL MISSION SUMMARY}

The Feed Delivery, Storage and Disposal Mission Summary (Figure 5) provides a graphical summary of the mission for Retrieval, Projects, and Operations related to feed delivery and return of products from the private contractors. This summary uses the information.from the Tank Waste Remediation System Operation and Utilization Plan (Kirkbride et al. 1997) and is consistent with the RTP schedules. 
HNF-1946 Rev. 0

This page intentionally left blank. 


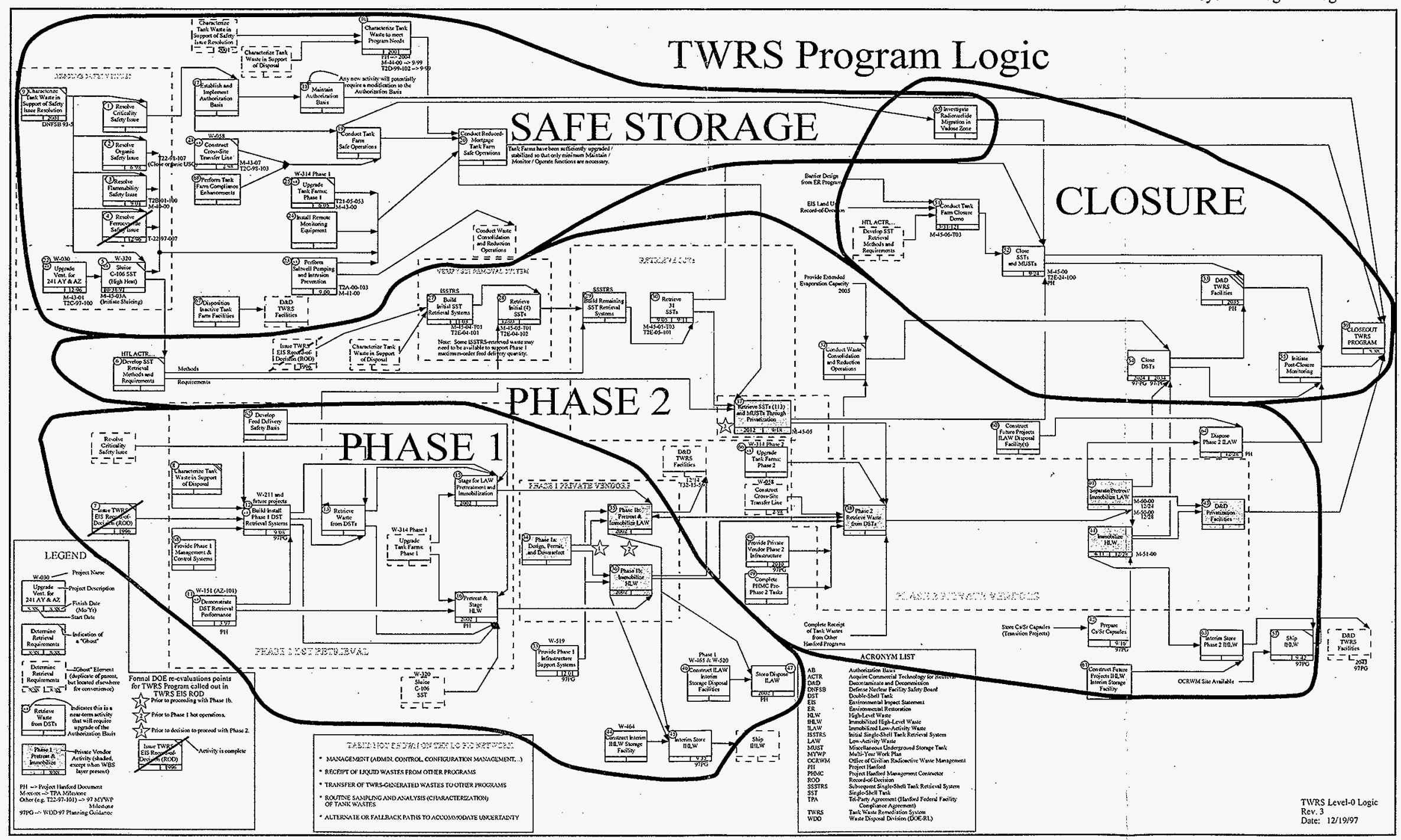




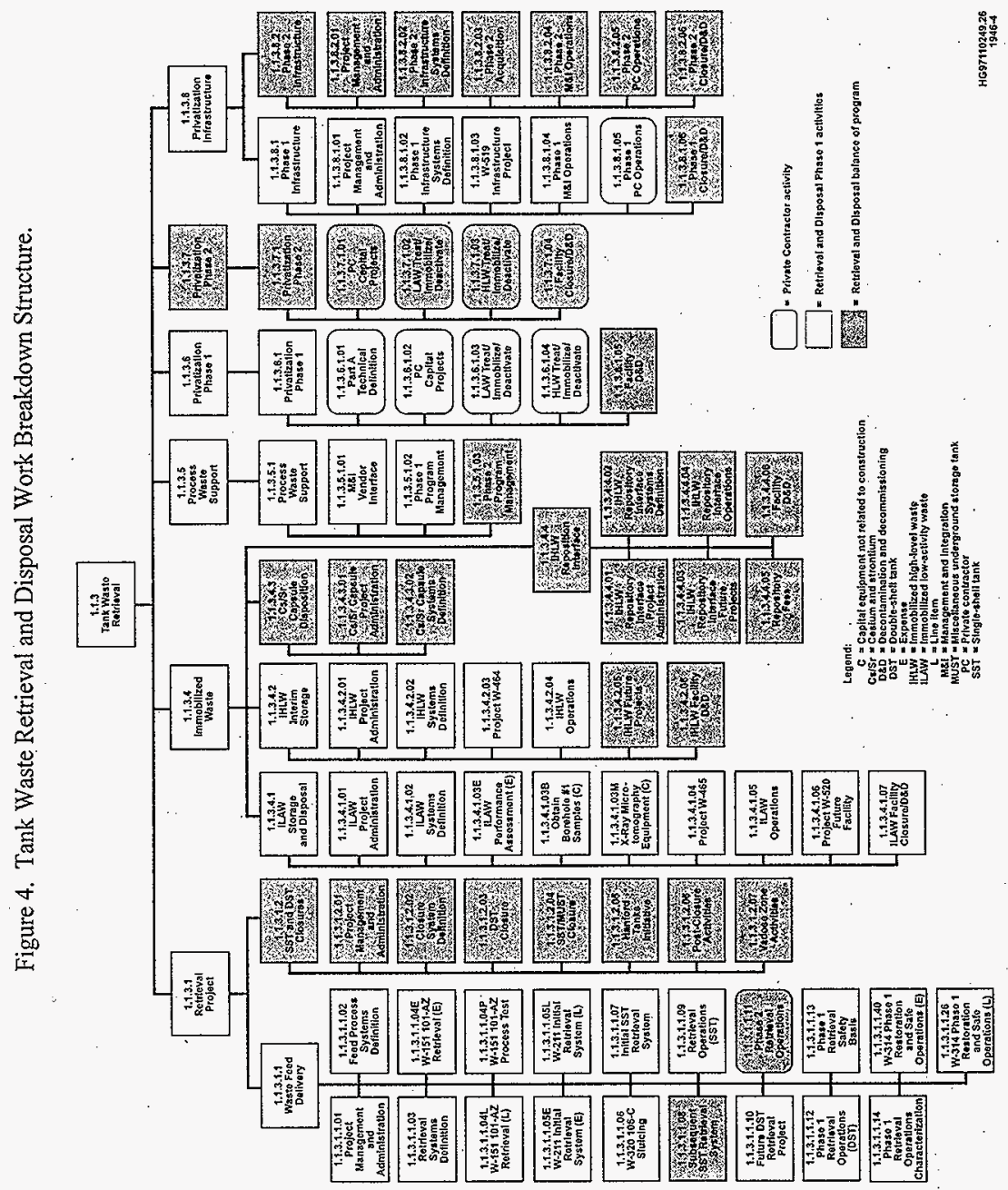


HNF-1946 Rev. 0

This page intentionally left blank. 


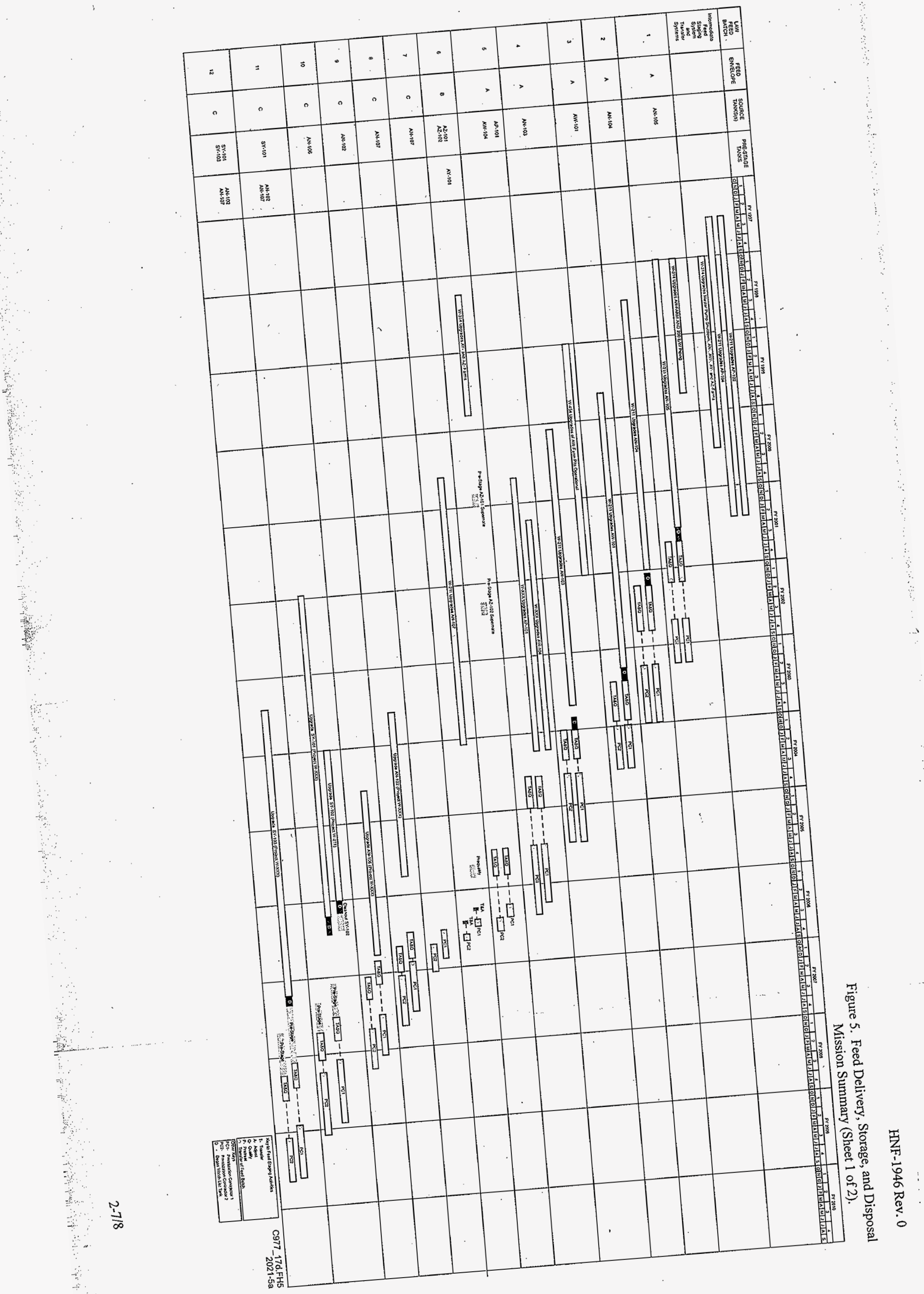




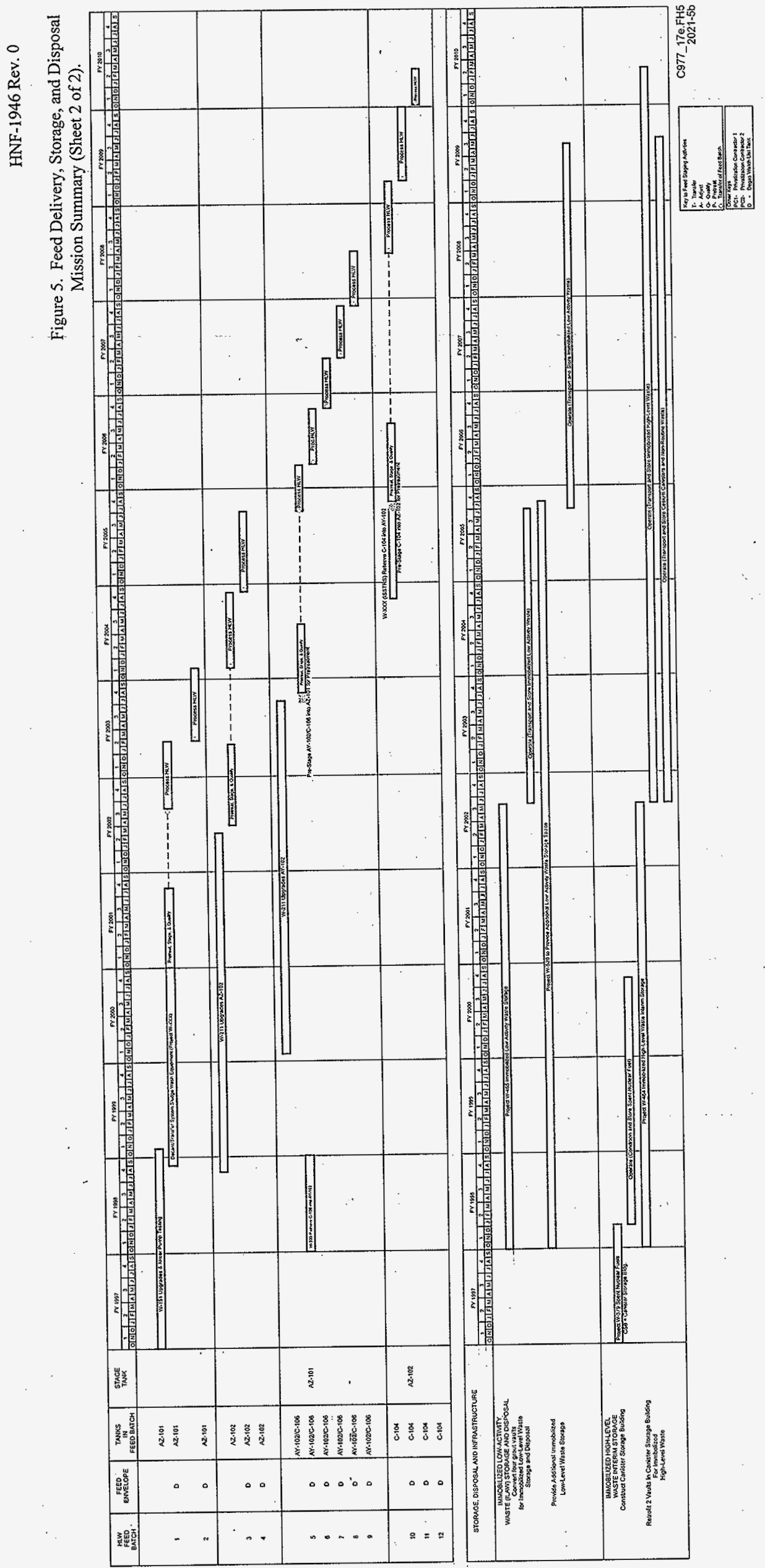




\subsection{LEVEL 1 LOGICS}

The Level 1 Logics were developed to define and detail the Phase 1 PHMC team scope of work and sequence of activities required to complete the TWRS Retrieval and Disposal Mission as reflected in the TWRS Retrieval and Disposal WBS. Level 1 Logics were prepared for LowActivity Waste Feed (11 sheets), High-Level Waste Feed (4 sheets), Privatization Infrastructure (1 sheet), and the Immobilized Waste (2 sheets). These Logics are located in Appendix A.

These logics were used as the basis for preparation of the TBR packages, which define and document the scope, schedule, and cost estimates, and are under configuration control. The Level 1 Logics identify primary activity owners including Environmental, Nuclear Safety and Licensing, Characterization, Retrieval, TWRS private contractors, Operations, Projects, and RL. A critical path for each feed batch is shown. Only selected logic activities for the TWRS private contractors and $\mathrm{RL}$ are shown in the Level 1 Logics to depict necessary interfaces. As discussed in Section 4.0, a limited number of Phase 1 TWRS Retrieval and Disposal Mission activities are not included in the Level 1 Logics.

\subsection{CROSSWALK FROM THE LEVEL 1 LOGIC TO THE WORK BREAKDOWN STRUCTURE}

This crosswalk (Table 1) shows the TWRS Retrieval and Disposal Level 1 Logic (activity number and title) crosswalked to the WBS Level 6.

\subsection{TWRS SCOPE/COST NOT INCLUDED IN THE INITIAL UPDATED BASELINE}

This section describes the TWRS scope/cost not included in the TWRS Retrieval and Disposal Mission Initial Updated Baseline. For a discussion of what costs are included, see Sections 4.1, Summary Cost Spreadsheet, and Section 4.2, Description of the Types of Cost. follows:

The TWRS scope/cost not included in the Initial Updated Baseline is broken down as

- Required Waste Management facilities base operations and interfaces are funded by the Waste Management Program (outside of TWRS):

- $\quad$ 222-S Laboratory support to tank characterization through FY 2034

- 242-A Evaporator support to AY/AZ Farm Sludge Washing and Dilute Wastes through FY 2013 
HNF-1946 Rev. 0

This page intentionally left blank. 
HNF-1946 Rev. 0

Table 1 Crosswalk from the Level 1 Logic to the Work Breakdown Structure

Level 1 Logic Level 6 WBS

Description

\begin{tabular}{|c|c|c|}
\hline 050.010 & 11310106 & W-320 Project Management \\
\hline 050.020 & 11310106 & Design New Eqpmnt - W-320 Includes C-106/AY-10 \\
\hline 050.030 & 11310106 & Construct New Eqpmnt - W-320 Includes C-106 \\
\hline 050.040 & 11310106 & Construct/ATP Original Equipment for W-320 \\
\hline 050.045 & 11310106 & Design/Equipment Support for W-320 \\
\hline 050.050 & 11310106 & Perform OTP for W-320 \\
\hline 050.060 & 11310106 & Prepare Safety Doc Basis for Interim Ops - W-320 \\
\hline 050.070 & 11310106 & Prep/Conduct ORR/Receive RL Approval - W-320 \\
\hline $050.079 \mathrm{~A}$ & 11310106 & M-45-03A Initiate Sluicing Retrieval of C-106 \\
\hline $050.079 \mathrm{~B}$ & 11310106 & Action for DNFSB 93-5, 5.4.3.6.C \\
\hline $050.079 \mathrm{C}$ & 11310106 & Start Retrieval Secretary Sfty Initiative W-320 \\
\hline 050.080 & 11310112 & Interface (xfr Part of AY-102 Supernatant Out) \\
\hline 050.085 & 11310106 & DOE-RL ORR for W-320 \\
\hline 050.090 & 11310106 & Sluic Sludge Into AY-102 - W-320. \\
\hline 050.100 & 11310106 & Design Additional Equipment W-320 \\
\hline 050.110 & 11310106 & Construct Additional Equipment W-320 \\
\hline 050.120 & 11310106 & Test Additional Equipment W-320 \\
\hline 050.130 & 11310106 & Process Control Document - W-320 \\
\hline 050.150 & 11310106 & Turnover Tank C-106 to HTI \\
\hline 110.010 & $1131014 \mathrm{E}$ & W-151 Project Management \\
\hline 110.010 & $1131014 \mathrm{P}$ & W-151 Project Management \\
\hline 110.030 & 11310106 & Apprd Safety Basis for Mixer Pump Perform Test \\
\hline 110.045 & $1131014 \mathrm{P}$ & Revise/Iss Mixer Pump Test Plans/Procedures \\
\hline 110.047 & 11310102 & Perf Mixer Pump Test Sampling \& Analysis \\
\hline 110.050 & $1131014 \mathrm{E}$ & Conduct LMHC Readiness Assessment \\
\hline 110.060 & $1131014 \mathrm{E}$ & DOE Approval to Operate \\
\hline 110.070 & $1131014 \mathrm{E}$ & Perform Mixer Pump Test (W-151) \\
\hline 110.070 & $1131014 \mathrm{P}$ & Perform Mixer Pump Test (W-151) \\
\hline 110.090 & $1131014 \mathrm{P}$ & Prepare Test Report (W-151) \\
\hline $120 . \mathrm{A} 00$ & $1131015 \mathrm{E}$ & W-211 Project Management \\
\hline 120.A00 & $1131015 \mathrm{~L}$ & W-211 Project Management \\
\hline 120.A88 & 11310110 & Prepare for $O R R A Z-101$ \\
\hline 120.B10 & 11310110 & Dsn/Install Central Control Room/Shift Ofc Facl \\
\hline $120 . \mathrm{B} 35$ & $1131015 \mathrm{~L}$ & W-211 Design AP-102/AP-104 Retrieval Systems \\
\hline $120 . \mathrm{B} 40$ & 11310112 & Prepare AP-102/AP-104 for Turnover to Project \\
\hline 120.B42 & $1131015 \mathrm{~L}$ & W-211 Equip Procure AP-102/AP-104 Retrieval Syst \\
\hline $120 . \mathrm{B} 45$ & $1131015 \mathrm{~L}$ & W-211 Construct AP-102/AP-104 Retrieval Systems \\
\hline $120 . \mathrm{B} 50$ & $1131015 \mathrm{E}$ & W-211 Startup AP-102/AP-104 Retrieval Systems \\
\hline 120.B58 & $1131015 \mathrm{E}$ & Prep for ORR on AP-102 \& 104 \\
\hline
\end{tabular}


HNF-1946 Rev. 0

Table 1 Crosswalk from the Level 1 Logic to the Work Breakdown Structure

Level 1 Logic Level 6 WBS

Description

\begin{tabular}{|c|c|c|}
\hline 120.B60 & $1131015 \mathrm{E}$ & Contract Independent ORR on AP-102 \& 104 \\
\hline 120.B65 & $1131015 \mathrm{E}$ & DOE ORR on AP-102 \& 104 \\
\hline $120 . \mathrm{C} 05$ & 11310102 & Obtain AY-101 Samples \\
\hline 120.C10 & 11310102 & Characterize AY-101 \\
\hline $120 . \mathrm{C} 12$ & 11310102 & Review AY-101 Historical Data \\
\hline $120 . \mathrm{Cl} 4$ & 11310102 & Establish Dilution Rqmts for AY-101 Process Test \\
\hline $120 . \mathrm{C} 15$ & 11310102 & Confirm Plans and Requirements for AY-101 \\
\hline 120.C20 & 11310102 & Update BBI \& TCR For Tank AY-101 \\
\hline $120 . \mathrm{C} 25$ & 11310103 & Evaluate Equipment Rqmts in AY-101 \\
\hline $120 . \mathrm{C} 30$ & 11310110 & Upgrade Waste Tank AY-101 as Required \\
\hline 120.007 & 11310102 & Develop LAW Confirm Tank T Meets Batch X DQO \\
\hline 120.D08 & 11310102 & Develop Waste Transfer to PC DQO \\
\hline 120.D09 & 11310102 & Develop In Tank Processing Control DQO \\
\hline 120.D10 & 11310102 & Evaluate AN-105 Samples for Adequate Matls \\
\hline 120.D11 & 11310102 & Develop DQO: PH 1 Tank Waste Transfer Control \\
\hline 120.D12 & 11310102 & Develop HLW Waste Delivery toPC DQO \\
\hline 120.015 & 11310102 & Rvw AN-105 Historical Data/Conf if Add'l Info Rq \\
\hline 120.D20 & 11310102 & Estab Dilution Reqmnts/Diss Behav for AN-105 \\
\hline 120.D25 & 11310102 & Characterize AN-105 \\
\hline 120.D30 & 11310102 & Update Best Basis Inventory and TCR for AN-105 \\
\hline $120 . \mathrm{D} 35$ & 11310102 & Perf Max Implied Radionuclide Concentrate Study \\
\hline 120.D40 & 11310102 & Confirm Plans and Requirements for AN-105 \\
\hline 120.D45 & 11310102 & Establish New Envelope Limits (A/B Versus C) \\
\hline 120.D50 & 11310102 & Do Envelopes Apply to Bulk Comp or Liquid Phase? \\
\hline 120.D55 & 11310102 & Establish Methods/Proc to Estab Feed Composition \\
\hline 120.D65 & $1131015 \mathrm{~L}$ & W-211 Design AN-105 Retrieval System \\
\hline $120 . D 72$ & $1131015 \mathrm{~L}$ & W-211 Equip Procurement AN-105 Retrieval System \\
\hline $120 . D 75$ & $1131015 \mathrm{~L}$ & W-211 Construction AN-105 Retrieval System \\
\hline 120.D80 & $1131015 \mathrm{E}$ & W-211 Startup AN-105 Retrieval System \\
\hline 120.D88 & $1131015 \mathrm{E}$ & Prep ORR AN-105 \\
\hline 120.D90 & $1131015 \mathrm{E}$ & Contract Independent ORR AN-105 \\
\hline 120.D95 & $1131015 \mathrm{E}$ & DOE ORR AN-105. \\
\hline 120.E05 & 11310102 & Evaluate AN-104 Samples for Adequate Matls \\
\hline 120.E10 & 11310102 & Estab Dilution Reqmnts/Diss Behav for AN-104 \\
\hline 120.E15 & 11310102 & Review AN-104 Historical Data \\
\hline 120.E20 & 11310102 & Characterize AN-104 \\
\hline 120.E25 & 11310102 & Update Best Basis Inventory and TCR for AN-104 \\
\hline 120.E35 & 11310102 & Confirm Plans and Requirements for AN-104 \\
\hline $120 . \mathrm{E} 40$ & 11310103 & Revise W-211 FDC for AN-104 \\
\hline
\end{tabular}


HNF-1946 Rev. 0

Table 1 Crosswalk from the Level 1 Logic to the Work Breakdown Structure

Description

\begin{tabular}{|c|c|c|}
\hline 120.E50 & $1131015 \mathrm{~L}$ & W-211 Design AN-104 Retrieval System \\
\hline $120 . \mathrm{E} 52$ & $1131015 \mathrm{~L}$ & W-211:Equip Procurement AN-104 Retrieval System \\
\hline 120.E55 & $1131015 \mathrm{~L}$ & W-211 Construction AN-104 Retrieval System \\
\hline 120.E60 & $1131015 \mathrm{E}$ & W-211 Startup AN-104 Mixing \& Retrieval System \\
\hline 120.E68 & $1131015 \mathrm{E}$ & Prep for Readiness Rvw on 241-AN-104 \\
\hline 120.E70 & $1131015 \mathrm{E}$ & Contractor Independent ORR for 241-AN-104 \\
\hline 120.E75 & $1131015 \mathrm{E}$ & DOE ORR for 241-AN-104 \\
\hline 120.F40 & $1131015 \mathrm{~L}$ & W-211 Design AZ-102 Retrieval System \\
\hline 120.F50 & $1131015 \mathrm{~L}$ & W-211 Construction AZ-102 Retrieval System \\
\hline 120.F52 & $1131015 \mathrm{~L}$ & W-211 Equip Procurement AZ-102 Retrieval System \\
\hline 120.F60 & $1131015 \mathrm{E}$ & W-211 Startup AZ-102 Retrieval System \\
\hline 120.F68 & $1131015 \mathrm{E}$ & Prepare for ORR AZ-102 \\
\hline 120.F80 & $1131015 \mathrm{E}$ & Contractor Independent ORR for AZ-102 \\
\hline 120.F90 & $1131015 \mathrm{E}$ & DOE ORR for AZ-102 \\
\hline 120.G05 & 11310102 & Evaluate AW-101 Samples Adequate Matls \\
\hline $120 . \mathrm{G} 10$ & 11310102 & Estab Dilution Reqmnts/Diss Behav for AW-101 \\
\hline 120.G15 & 11310102 & Review AW-101 Historical Data \\
\hline $120 . \mathrm{G} 20$ & 11310102 & Characterize AW-101 \\
\hline $120 . \mathrm{G} 25$ & 11310102 & Update Best Basis Inventory and TCR for AW-101 \\
\hline $120 . G 35$ & 11310102 & Confirm Plans and Requirements for AW-101 \\
\hline $120 . \mathrm{G} 37$ & 11310102 & Eval Waste Retrieyal Equip Req'd for Tanks 5 On \\
\hline $120 . \mathrm{G} 38$ & 11310103 & Decn Board/Acqn Strategy PHI Ret After AN-104. \\
\hline $120 . \mathrm{G} 39$ & 11310103 & Adjust Acquistion Strategy WFD System \\
\hline $120 . \mathrm{G} 40$ & 11310103 & Revise W-211 FDC for AW-101 \\
\hline $120 . \mathrm{G} 50$ & $1131015 \mathrm{~L}$ & W-211 Design AW-101 Retrieval System \\
\hline $120 . \mathrm{G} 52$ & $1131015 \mathrm{~L}$ & W-211 Equip Procurement AW-101 Retrieval System \\
\hline 120.G55 & $1131015 \mathrm{~L}$ & W-211 Construction AW-101 Retrieval System \\
\hline $120 . \mathrm{G} 60$ & $1131015 \mathrm{E}$ & W-211 Startup AW-101 Retrieval System \\
\hline 120.668 & $1131015 \mathrm{E}$ & Prep for ORR on AW-101 \\
\hline $120 . G 70$ & $1131015 \mathrm{E}$ & Contract Independent ORR on AW-101 \\
\hline $120 . \mathrm{G} 75$ & $1131015 \mathrm{E}$ & DOE ORR on AW-101 \\
\hline 120:H10 & $1131015 \mathrm{~L}$ & W-211 Design AY-102 Retrieval System \\
\hline 120.H15 & $1131015 \mathrm{~L}$ & W-211 Equip Procurement AY-102 Retrieval System \\
\hline $120 . \mathrm{H} 20$ & $1131015 \mathrm{~L}$ & W-211 Construction AY-102 Retrieval System \\
\hline $120 . \mathrm{H} 30$ & $1131015 \mathrm{E}$ & W-211 Startup AY-102 Retrieval System \\
\hline 120.H50 & $1131015 \mathrm{E}$ & Contractor Independent ORR AY-102 \\
\hline 120.H60 & $1131015 \mathrm{E}$ & DOE ORR AY-102 \\
\hline $120 . \mathrm{H} 68$ & $1131015 \mathrm{E}$ & Prepare for ORR AY-102 \\
\hline 120.J05 & 11310102 & Evaluate AN-103 Samples for Adequate Matls \\
\hline
\end{tabular}


HNF-1946 Rev. 0

Table 1 Crosswalk from the Level 1 Logic to the Work Breakdown Structure

Description

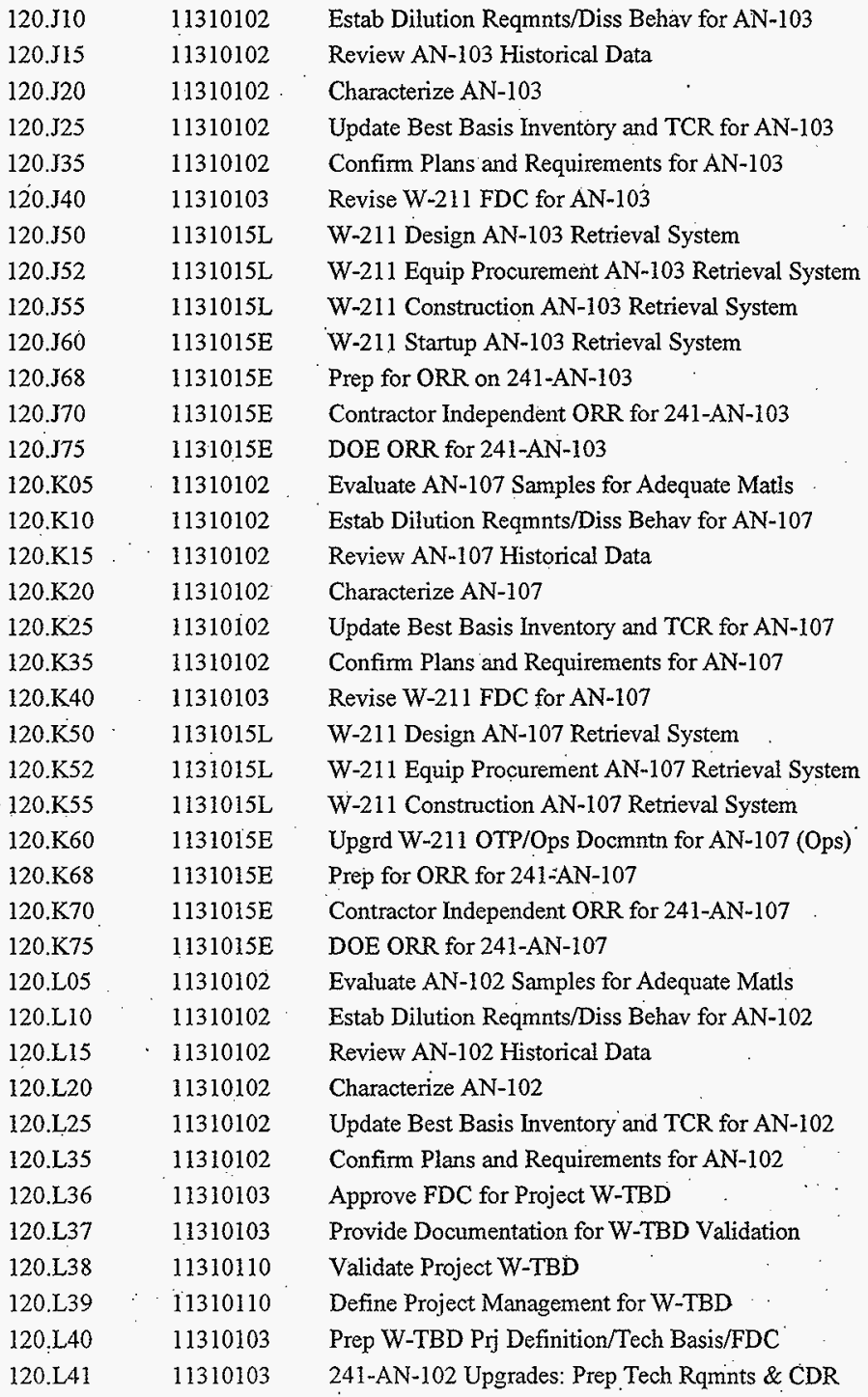


HNF-1946 Rev. 0

Table 1 Crosswalk from the Level 1 Logic to the Work Breakdown Structure Level 1 Logic Level 6 WBS

Description

\begin{tabular}{|c|c|c|}
\hline $120 . L 50$ & 11310110 & 241-AN-102 Upgrades: Design \& Enviro. Permits \\
\hline 120.L55 & 11310110 & 241-AN-102 Upgrades: Const/ATP/Tumover/H14 Dwgs \\
\hline 120.L60 & 11310110 & 241-AN-102 Upgrades: Startup/OTP/Ops Documents \\
\hline $120 . \mathrm{L} 68$ & 11310110 & Prep for ORR for $241-A N-102$ \\
\hline 120.270 & 11310110 & Contractor Independent ORR for $241-\mathrm{AN}-102$ \\
\hline 120.L75 & 11310110 & DOE ORR for 241-AN-102 \\
\hline 120.M05 & 11310102 & Evaluate AN-106 Samples for Adequate Matls \\
\hline 120.M10 & 11310102 & Estab Dilution Reqmnts/Diss Behav for AN-106 \\
\hline 0.M15 & 11310102 & Review AN-106 Historical Data \\
\hline $0 . \mathrm{M} 20$ & 11310102 & Characterize AN-106 \\
\hline $20 . \mathrm{M} 25$ & 11310102 & Update Best Basis Inventory and TCR for AN-106 \\
\hline 20.M35 & 11310102 & Confirm Plans and Requirements for AN-106 \\
\hline 20.M40 & 11310103 & 241-AN-106 Upgrades: Prep Tech Rqmnts \& CDR \\
\hline 120.M50 & 11310110 & 241-AN-106 Upgrades: Design \& Enviro. Permits \\
\hline 120.M55 & 11310110 & 241-AN-106 Upgrades: Const/ATP/Tumover/H14 Dwgs \\
\hline 120.M60 & 11310110 & 241-AN-106 Upgrades: Startup/OTP/Ops Documents \\
\hline 120.M68 & 11310110 & Prep for ORR on Tank 241-AN-106 \\
\hline 120.M70 & 11310110 & Contractor Independent ORR for 241-AN-106 \\
\hline 120.M75 & 11310110 & DOE ORR for 241-AN-106 \\
\hline 120.P22 & $1131015 \mathrm{E}$ & Contractor Independent ORR for SY-102 \\
\hline 120.P25 & $1131015 \mathrm{E}$ & DOE Operational Readiness Review for SY-102 \\
\hline $120 . \mathrm{P} 32$ & 11310103 & Validate SY-102 Design to DST Specification \\
\hline 120.P60 & $1131015 \mathrm{~L}$ & W-211 Construction SY-102 Retrieval System \\
\hline 120.P70 & $1131015 \mathrm{E}$ & W-211 Startup SY-102 Retrieval System \\
\hline 120.P90 & $1131015 \mathrm{E}$ & Prep for ORR on 241-SY-102 Project W-211 \\
\hline 120.R05 & 11310102 & Evaluate SY-101 Samples for Adequate Matls \\
\hline 120.R10 & 11310102 & Estab Dilution Reqmints/Diss Behav for SY-101 \\
\hline 120.R15. & 11310102 & Review SY-101 Historical Data \\
\hline 120.R20 & 11310102 & Characterize SY-101 \\
\hline 120.R25 & 11310102 & Update Best Basis Inventory and TCR for SY-101 \\
\hline $120 . \mathrm{R} 35$ & 11310102 & Confirm Plans and Requirements for SY-101 \\
\hline 120.R40 & 11310103 & Revise W-TBD Design Specifications for SY-101 \\
\hline 120.R50 & 11310110 & Design Upgrade W-TBD for SY-101 \\
\hline 120.R52 & 11310110 & Equipment Procurement for SY-101 \\
\hline 120.R55 & 11310110 & Construct/Test/Project Tumover of SY-101 \\
\hline $120 . R 60$ & 11310110 & Startup for SY-101 (W-TBD) Mixing \& Retr Sys \\
\hline 120.R68 & 11310110 & Prep for ORR on $241-S Y-101$ \\
\hline 120.R70 & 11310110 & Contract Independent ORR on 241-SY-10I \\
\hline $120 . R 75$ & 11310110 & DOE ORR on $241-S Y-101$ \\
\hline
\end{tabular}


HNF-1946 Rev. 0

Table 1 Crosswalk from the Level 1 Logic to the Work Breakdown Structure Level 1 Logic Level 6 WBS

Description

\begin{tabular}{|c|c|c|}
\hline 120.T05 & 11310102 & Evaluate SY-103 Samples for Adequate Matls \\
\hline $120 . T 10$ & 11310102 & Estab Dilution Reqmnts/Diss Behav for SY-103 \\
\hline $20 . \mathrm{T} 15$ & 11310102 & Review SY-103 Historical Data \\
\hline $0 . T 20$ & 11310102 & Characterize SY-103 \\
\hline $0 . T 25$ & 11310102 & Update Best Basis Inventory and TCR for SY-103 \\
\hline 0.735 & 11310102 & Confirm Plans and Requirements for SY -103 \\
\hline $0 . T 40$ & 11310103 & Revise W-TBD Design Specifications for SY-103 \\
\hline 20.750 & 11310110 & Design Upgrade W-TBD for SY-103. \\
\hline 20.752 & 11310110 & Equipment Procurement W-TBD for SY-103 \\
\hline 20.T55 & 11310110 & Construct/Test/Project Turnover of SY-103 \\
\hline 20.560 & 11310110 & Startup for SY-103 (W-TBD) Mixing \& Retr Sys \\
\hline 20.568 & 11310110 & Prep for ORR on SY -103 \\
\hline $20 . T 70$ & 11310110 & Contract Independent ORR on SY-103 \\
\hline 20.775 & 11310110 & DOE ORR on SY-103 \\
\hline $20 . \mathrm{V} 05$ & 11310102 & Obtain AP-101 Samples \\
\hline 20.V10 & 11310102 & Characterize AP-101 \\
\hline $20 . \mathrm{V} 12$ & 11310102 & Review AP-101 Historical Data \\
\hline 20.V14 & 11310102 & Establish Dilution Rqmts for AP-101 Process Test \\
\hline $120 . V 15$ & 11310102 & Confirm Plans and Requirements for AP-101 \\
\hline 120.V20 & 11310102 & Update BBI \& TCR For Tank AP-I01 \\
\hline $20 . V 25$ & 11310103 & Evaluate Equipment Rqmts in AP-101 \\
\hline 20.V30 & 11310110 & Upgrade Waste Tank AP-101 as Required \\
\hline 20.W05 & 11310102 & Obtain AW-I04 Samples \\
\hline 20.W10 & 11310102 & Characterize AW-104 \\
\hline $20 . \mathrm{W} 12$ & 11310102 & Review AW-104 Historical Data \\
\hline $0 . W 14$ & 11310102 & Establish Dilution Rqmts for AW-104 Process Test \\
\hline 20.W15 & 11310102 & Confirm Plans and Requirements for AW-104 \\
\hline 20.W20 & 11310102 & Update BBI \& TCR For Tank AW-104 \\
\hline 20.W25 & 11310103 & Evaluate Equipment Rqmts in AW-104 \\
\hline $120 . W 30$ & 11310110 & Upgrade Waste Tank AW-104 as Required \\
\hline $120 . \mathrm{X01}$ & 11350101 & DOE-RL Private Contr Samp \& Data Requiments \\
\hline 130.010 & 11310102 & Phase I Tank Waste Transfer Control \\
\hline 130.015 & 11310112 & Manage Waste Transfer System During Xfer Sys Upg \\
\hline $130 . \mathrm{B} 05$ & 11310102 & ID Reqd Spares WFD 1st Batches LAW \& LAW \\
\hline 130.B10 & 11310112 & Procure WFD Spare Equip (Operations) \\
\hline $130 . \mathrm{B} 15$ & 11310112 & Prep to Maintain the WFD Sys Operable \\
\hline $130 . \mathrm{B} 16$ & 11310112 & Maintain WFD Sys Operable \\
\hline $130 . \mathrm{B} 17$ & 11310113 & Devel Tech Basis: Rem Waste F/Watchlist Tanks \\
\hline $130 . \mathrm{B} 20$ & 11310113 & Obtain Permission to Add Diluent to FGWL DSTs \\
\hline
\end{tabular}


HNF-1946 Rev. 0

Table 1 Crosswalk from the Level 1 Logic to the Work Breakdown Structure Level 1 Logic Level 6 WBS

Description

\begin{tabular}{|c|c|c|}
\hline $130 . \mathrm{B} 25$ & 11310112 & Degas AN-105 \\
\hline 130.830 & 11310112 & $\mathrm{Mob} /$ Retr Wst in AN-105/Rec Feed in AP-102/AP-104 \\
\hline $130 . \mathrm{B} 40$ & 11310112 & Perform B்ootstrap Transfers. \\
\hline $130 . \mathrm{B} 45$ & 11310102 & Prepare for Sampling During LAW Feed Staging \\
\hline $30 . \mathrm{B} 55$ & 11310112 & Prepare Batch 1 Process Control Plan AN-105 \\
\hline 130.B62 & 11310110 & Úpgrd Valve Pits AW-A\&B - W-454-Replace Jumpers \\
\hline 30.865 & 11310101 & TWRS Management Assessment (LAW) \\
\hline 0.B70 & 11310101 & Prepare Plan/Document for Mgmt Assessment (LAW) \\
\hline 0.875 & 11310102 & Compare RAM/O\&M Concept w/ OTP Results \\
\hline $30 . \mathrm{B} 80$ & 11310102 & Assess Sec. Waste Util \& Samp Anly Agnst Plans \\
\hline $30 . \mathrm{C} 10$ & 11310112 & Retrieve Waste in AY-101/Receive Feed in AP-102 \\
\hline $30 . \mathrm{C} 20$ & 11310112 & Retrieve Waste in AY-101/Receive Feed in AP-104 \\
\hline $130 . C 25$ & 11310112 & Prepare AY-101 Process Control Plan \\
\hline 130.E10 & 11310112 & Degas AN-104 \\
\hline $130 . \mathrm{E} 25$ & 11310112 & Prepar AN-104 Process Control Plan \\
\hline 30.E30 & 11310112 & Mob/Retr Wst in AN-104/Rec Feed in AP-102/104 \\
\hline 130.F10 & 11310112 & B.S. Xfer AZ-102 Supernate into AY-101/AN-104 \\
\hline 130.F20 & 11310112 & Prepare AZ-102 PRocess Control Plan \\
\hline $30 . G 10$ & 11310112 & Degas AW-101 \\
\hline $130 . \mathrm{G} 25$ & 11310112 & Prepare AW-101 Procoess Control Plan \\
\hline $130 . \mathrm{G} 30$ & 11310112 & Mob/Retr Wst in AW-101/Rec Feed in AP-102 \\
\hline $130 . \mathrm{G} 40$ & 11310112 & Transfer 2nd Vendor Waste From AW-101 to AP-104 \\
\hline 130.H10 & 11310112 & Bootstrap Trans AY-102 Supernate into AN-105 \\
\hline $130 . \mathrm{H} 20$ & 11310112 & Prepare Tank 241-AY-102 Process Control Plan \\
\hline 130.J10 & 11310112 & Degas AN-103 \\
\hline 130.J20 & 11310112 & Prepare C-104/AZ-101 Process Control Plan \\
\hline $30 . \mathrm{J} 25$ & 11310112 & Prepare AN-103 Process Control Plan \\
\hline $130 . \mathrm{J} 30$ & 11310112 & Transfer Wst in AN-103/Rec Feed in AP-102 \\
\hline $130 . J 40$ & 11310112 & Transfer Wst in AN-103/Rec Feed in AP-104 \\
\hline $130 . \mathrm{K} 20$ & 11310112 & Prepare AN-107 Process Control Plan \\
\hline $130 . \mathrm{K} 30$ & 113101.12 & Transfer Wst in AN-107/Rec Feed in AP-102 \\
\hline 130.K40 & 11310112 & Transfer Wst in AN-107/Rec Feed in AP-104 \\
\hline 130.L20 & 11310112 & Prepare AN-102 Process Control Plan \\
\hline $130 . \mathrm{L} 30$ & 11310112 & Transfer Wst in AN-102/Rec Feed in AP-102 \\
\hline 130.L40 & 11310112 & Transfer Wst in AN-102/Rec Feed in AP-104 \\
\hline 130.M20 & 11310112 & Prepare AN-106 Process Control Plan \\
\hline 130:M30 & 11310112 & Transfer Wst in AN-106/Rec Feed in AP-102 \\
\hline 30.M40 & 11310112 & Transfer Wst in AN-106/Rec Feed in AP-104 \\
\hline $30 . \mathrm{N} 10$ & 11310102 & Prep for Sampling During HLW Feed Staging AZ-101 \\
\hline
\end{tabular}


HNF-1946 Rev, 0

Table 1 Crosswalk from the Level 1 Logic to the Work Breakdown Structure

Level 1 Logic Level 6 WBS

Description

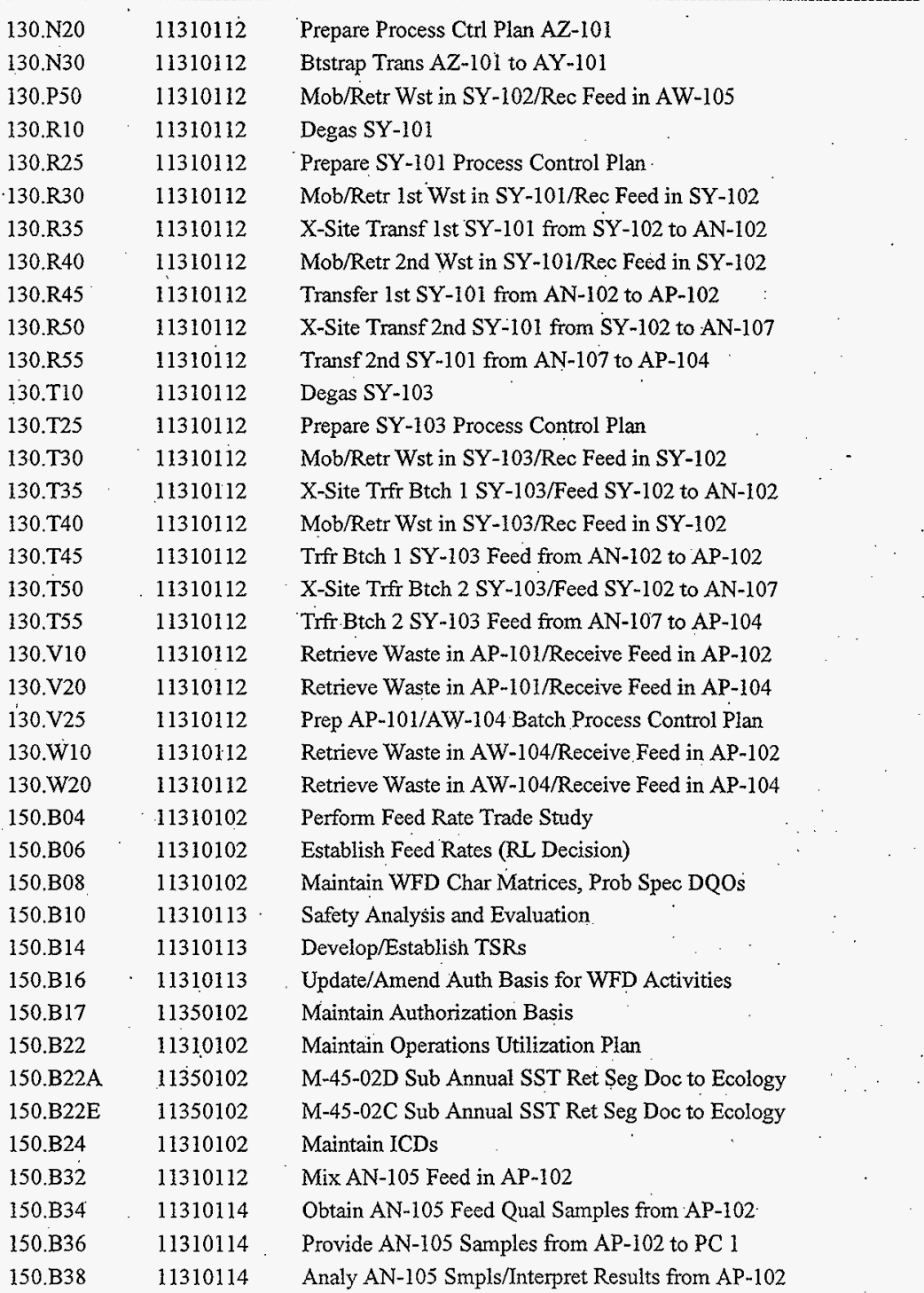


HNF-1946 Rev. 0

Table 1 Crosswalk from the Level 1 Logic to the Work Breakdown Structure

Level 1 Logic Level 6 WBS

Description

\begin{tabular}{|c|c|c|}
\hline $150 . \mathrm{B} 40$ & 11310114 & Prov AN-105 Feed Batch Info from AP-102 to PC 1 \\
\hline $150 . \mathrm{B} 42$ & 11310112 & Adjust AN-105 Feed in AP-102 \\
\hline 150.B44 & 11310112 & Deliver AN-105 Feed to AP-106 \\
\hline $150 . \mathrm{B} 46 \mathrm{H}$ & 11350112 & TWRS EIS ROD Hold Point 2 \\
\hline 150.848 & 11310112 & Mix AN-105 Feed in AP-104 \\
\hline $150 . \mathrm{B} 50$ & 11310114 & Obtain AN-105. Feed Qual Samples from AP-104 \\
\hline $150 . \mathrm{B} 52$ & 11310114 & Provide AN-105 Samples from AP-104 to PC 2 \\
\hline 150.B54 & 11310114 & Analy AN-105 Smpls/Interpret Results from AP-104 \\
\hline 150.B56 & 11310114 & Provide AN-105 Feed Batch Info to PC 2 \\
\hline $150 . \mathrm{B} 58$ & 11310112 & Adjust AN-105 Feed in AP-104 \\
\hline $150 . \mathrm{B} 60$ & 11310112 & Deliver AN-105 Feed to AP-108 \\
\hline $150 . \mathrm{B} 63 \mathrm{H}$ & 11350112 & TWRS EIS ROD Hold Point 1 \\
\hline $150 . \mathrm{B} 66$ & 11380102 & Ready PHMC Oper/Maint Doc-AP-108 Turnover \\
\hline $150 . \mathrm{B} 68$ & 11350112 & Execute Tank Turnover Procedure - PC 2 \\
\hline 150.870 & 11350112 & RL Declares AP-108 Ready - Contractor 2 \\
\hline 150.B76 & 11380102 & Ready PHMC Oper/Maint Doc-AP-106 Tumover \\
\hline $150 . \mathrm{B} 78$ & 11350112 & Execute Tank Turnover Procedure - PC 1 \\
\hline $150 . \mathrm{B} 80$ & 11350112 & RL Declares AP-106 Ready - Contractor 1 \\
\hline $150 . \mathrm{B} 82$ & 11310102 & Perform Feed Tank Fill Status Trade Study \\
\hline $150, \mathrm{~B} 84$ & 11310102 & Establish Feed Tank Fill Status (RL Decision) \\
\hline $150 . \mathrm{B} 86$ & 11350112 & Final ICD for Waste Feed Tank AP-106 - PC 1 \\
\hline $150.888^{\circ}$ & 11380102 & Prep AP-106 for Tumover to Private Contractor \\
\hline 150.B90 & 11350112 & Final ICD for LAW Feed - PC 2 \\
\hline $150 . \mathrm{B} 92$ & 11350112 & Final ICD for LAW Feed - PC 1 \\
\hline $150 . \mathrm{B} 94$ & 11380102 & Establish Interface Reqmnts for Tank Turnover \\
\hline 150.896 & 11380102 & Final ICD for Waste Feed Tank AP-108 - PC 2 \\
\hline 150.B98 & 11380102 & Prep AP-108 for Tumover to Private Contractor \\
\hline $150 . \mathrm{CO} 5$ & 11310112 & Mix AY-101 Feed in AP-102 \\
\hline $150 . \mathrm{C} 10$ & 11310114 & Obtain AY-101 Feed Qual Samples from AP-102 \\
\hline $150 . \mathrm{C} 15$ & 11310114 & Provide AY-101 Samples from AP-102 to PC 1 \\
\hline $150 . \mathrm{C} 20$ & 11310114 & Analy AY-101 Smpls/Interpret Results from AP-102 \\
\hline $150 . \mathrm{C} 25$ & 11310114 & Provide AY-101 Feed Batch Info to PC 1 \\
\hline $150 . C 30$ & 11310112 & Adjust AY-101 Feed in AP-102 \\
\hline $150 . \mathrm{C} 35$ & 11310112 & Deliver AY-101 Feed from AP-102 to AP-106 \\
\hline $150 . C 40$ & 11310112 & Mix AY-101 Feed in AP-104 \\
\hline $150 . \mathrm{C} 45$ & 11310114 & Obtain AY-101 Feed Qual Samples from AP-104 \\
\hline $150 . \mathrm{C} 50$ & 11310114 & Provide AY-101 Samples from AP-104 to PC 2 \\
\hline $150 . C 55$ & 11310114 & Analy AY-101 Smpls/lnterpret Results from AP-104 \\
\hline $150 . \mathrm{C} 60$ & 11310114 & Prov AY-101 Feed Batch Info from AP-104 to PC 2 \\
\hline
\end{tabular}


HNF-1946 Rev: 0

Table 1 Crosswalk from the Level 1 Logic to the Work Breakdown Structure Level I Logic Level 6 WBS Description

\begin{tabular}{|c|c|c|c|}
\hline $150 . \mathrm{C} 65$ & & 11310112 & Adjust AY-101 Feed in AP-104 \\
\hline 150.C70 & & 11310112 & Deliver AY-101 Feed from AP-104 to AP-108 \\
\hline $150 . \mathrm{E} 05$ & & 11310112 & Mix AN-104 Feed in AP-102 \\
\hline 50.E10 & & 11310114 & Obtain AN-104 Feed Qual Samples from AP-102 \\
\hline 150.E15 & & 11310114 & Provide AN-104 Samples from AP-102 to PC 1 \\
\hline 150.E20 & & 11310114 & Analy AN-104 Smpls/Interpret Results from AP-102 \\
\hline $150 . \mathrm{E} 25$ & & 11310114 & Provide AN-104 Feed Batch Info to PC 1 \\
\hline 150.E30 & & 11310112 & Adjust AN-104 Feed in AP-102 \\
\hline 150.E35 & & 11310112 & Deliver AN-104 Feed from AP-102 to AP-106 \\
\hline 50.E40 & & 11310112 & Mix AN-104 Feed in AP-104 \\
\hline 50.E45 & & 11310114 & Obtain AN-104 Feed Qual Samples from AP-104 \\
\hline 150.E50 & & 11310114 & Provide AN-104 Samples from AP-104 to PC 2 \\
\hline 150.E55 & & 11310114 & Analy AN-104 Smpls/Interpret Results from AP-104 \\
\hline 150.E60 & & 11310114 & Prov AN-104 Feed Batch Info from AP-104 to PC 2 \\
\hline 150.E64 & & 11310112 & Empty AP-107 Into AN-104 for Vendor Returns \\
\hline 150.E65 & & 11310112 & Adjust AN-104 Feed in AP-104 \\
\hline 150.E70 & & 11310112 & Deliver AN-104 Feed from AP-104 to AP-108 \\
\hline $150 . \mathrm{G} 05$ & & .11310112 & Mix AW-101 Feed in AP-102 \\
\hline $150 . G 10$ & & 11310114 & Obtain AW-101 Feed Qual Samples from AP-102 \\
\hline $150 . \mathrm{G} 15$ & & 11310114 & Provide AW-101 Samples from AP-102 to PC 1 \\
\hline $150 . \mathrm{G} 20$ & $\cdot$ & 11310114 & Analy AW-101 Smpls/Interpret Results from AP-102 \\
\hline $150 . G 25$ & $\cdot$ & 11310114 & Prov AW-101 Feed Batch Info from AP-102 to PC 1 \\
\hline $150 . \mathrm{G} 30$ & & 11310112 & Adjust AW-101 Feed in AP-102 \\
\hline $150 . \mathrm{G} 35^{\circ}$ & & 11310112 & Deliver AW-101 Feed from AP-102 to AP-106 \\
\hline $150 . \mathrm{G} 40$ & & 11310112 & Mix AW-101 Feed in AP-104 \\
\hline $150 . \mathrm{G} 45$ & & 11310114 & Obtain AW-101 Feed Qual Samples from AP-104 \\
\hline $150 . \mathrm{G} 50$ & & 11310114 & Provide AW-101 Samples from AP-104 to PC 2 \\
\hline $150 . \mathrm{G} 55$ & & 11310114 & Analy AW-101 Smpls/Interpret Results from AP-104 \\
\hline $150 . \mathrm{G} 60$ & & 11310114 & Prov AW-101 Feed Batch Info from AP-104 to PC 2 \\
\hline $150 . \mathrm{G} 65$ & & 11310112 & Adjust AW-101 Feed in AP-104 \\
\hline $150 . G 70$ & & 11310112 & Deliver AW-101 Feed from AP-104 to AP-108 \\
\hline 150.J05 & & 11310112 & Mix AN-103 Feed in AP-102 \\
\hline $150 . J 10$ & & 11310114 & Obtain AN-103 Feed Qual Samples from AP-102 \\
\hline $150 . J 15$ & & 11310114 & Provide AN-103 Samples from AP-102 to PC 1 \\
\hline $150 . \mathrm{J} 20$ & & 11310114 & Analy AN-103 Smpls/Interpret Results from AP-102 \\
\hline $150 . J 25$ & & 11310114 & Provide AN-103 Feed Batch Info to PC 1 \\
\hline 150.J30 & & 11310112 & Adjust AN-103 Feed in AP-102 \\
\hline $150 . \mathrm{J} 35$ & & 11310112 & Deliver AN-103 Feed from AP-102 to AP-106 \\
\hline $150 . \mathrm{J} 40$ & & 11310112 & Mix AN-103 Feed in AP-104 \\
\hline
\end{tabular}


HNF-1946 Rev. 0

Table 1 Crosswalk from the Level 1 Logic to the Work Breakdown Structure Level 1 Logic Level 6 WBS

Description

\begin{tabular}{|c|c|c|}
\hline $150 . J 45$ & 11310114 & Obtain AN-103 Feed Qual Samples from AP-104 \\
\hline $150 . J 50$ & 11310114 & Provide AN-103 Samples from AP-104 to PC 2 \\
\hline 150.355 & 11310114 & Analy AN-103 Smpls/Interpret Results from AP-104 \\
\hline 0.360 & 11310114 & Prov AN-103 Feed Batch Info from AP-104 to PC 2 \\
\hline $50 . \mathrm{J} 65$ & 11310112 & Adjust AN-103 Feed in AP-104 \\
\hline ;0.J70 & $11310112^{\circ}$ & Deliver AN-103 Feed from AP-104 to AP-108 \\
\hline $0 . \mathrm{K} 05$ & 1.1310112 & Mix AN-107 Feed in AP-102 \\
\hline $50 . K 10$ & 11310114 & Obtain AN-107 Feed Qual Samples from AP-102 \\
\hline $50 . \mathrm{K} 15$ & 11310114 & Provide AN-107 Samples from AP-102 to PC 1 \\
\hline $150 . \mathrm{K} 20$ & 11310114 & Analy AN-107 Smpls/Interpret Results from AP-102 \\
\hline $50 . \mathrm{K} 25$ & 11310114 & Prov AN-107 Feed Batch Info from AP-102 to PC 1 \\
\hline 50.K30 & 11310112 & Adjust AN-107 Feed \\
\hline $0 . \mathrm{K} 35$ & 11310112 & Deliver AN-107 Feed from AP-102 to AP-106 \\
\hline $50 . \mathrm{K} 37$ & 11310112 & Deliver AN-107 Feed from AP-102 to AP-106 \\
\hline 50.K70 & 11310112 & Deliver AN-107 Feed from AP-104 to AP-108 \\
\hline $50 . \mathrm{K} 72$ & 11310112 & Deliver AN-107 Feed from AP-104 to AP-108 \\
\hline 50.L05 & 11310112 & Mix AN-102 Feed in AP-102 \\
\hline 150.L10 & 11310114 & Obtain AN-102 Feed Qual Samples from AP-102 \\
\hline 150.L15 & 11310114 & Provide AN-102 Samples from AP-102 to PC 1 \\
\hline 150.L20 & 11310114 & Analy AN-102 Smpls/Interpret Results from AP-102 \\
\hline 150.L25 & 11310114 & Prov AN-102 Feed Batch Info from AP-102 to PC 1 \\
\hline $150 . L 30$ & 11310112 & Adjust AN-102 Feed in AP-102 \\
\hline $150 . \mathrm{L35}$ & 11310112 & Deliver AN-102 Feed from AP-102 to AP-106 \\
\hline 150.L40 & 11310112 & Mix AN-102 Feed in AP-104 \\
\hline 150.L45 & 11310114 & Obtain AN-102 Feed Qual Samples from AP-104. \\
\hline 150.L50 & 11310114 & Provide AN-102 Samples from AP-104 to PC 2 \\
\hline 150.L55 & 11310114 & Analy AN-102 Smpls/Interpret Results from AP-104 \\
\hline 150.L60 & 11310114 & Provi AN-102 Feed Batch Info from AP-104 to PC 2 \\
\hline 150.L65 & 11310112 & Adjust AN-102 Feed in AP-104 \\
\hline $150 . \mathrm{L} 70$ & 11310112 & Deliver AN-102 Feed from AP-104 to AP-108 \\
\hline 150.M05 & 11310112 & Mix AN-106 Feed in AP-102 \\
\hline $150 . \mathrm{M} 10$ & 11310114 & Obtain AN-106 Feed Qual Samples from AP-102 \\
\hline 150.M15 & 11310114 & Provide AN-106 Samples from AP-102 to PC 1 . \\
\hline 150.M20 & 11310114 & Analy AN-106 Smpls/Interpret Results from AP-102 \\
\hline $150 . \mathrm{M} 25$ & 11310114 & Prov AN-106 Feed Batch Info from AP-102 to PC 1 \\
\hline $150 . \mathrm{M} 30$ & 11310112 & Adjust AN-106 Feed in AP-102 \\
\hline $150 . M 35$ & 11310112 & Deliver AN-106 Feed from AP-102 to AP-106 \\
\hline $150 . \mathrm{M} 40$ & 11310112 & Mix AN-106 Feed in AP-104 \\
\hline 150.M45 & 11310114 & Obtain AN-106 Feed Qual Samples from AP-104 \\
\hline
\end{tabular}


HNF-1946 Rev. 0

Table 1 Crosswalk from the Level 1 Logic to the Work Breakdown Structure Level 1 Logic Level 6 WBS

Description

\begin{tabular}{|c|c|c|}
\hline 150.M50 & 11310114 & Provide AN-106 Samples from AP-104 to PC 2 \\
\hline 150.M55 & 11310114 & Analy AN-106 Smpls/Interpret Results from AP-104 \\
\hline 150.M60 & 11310114 & Prov AN-106 Feed Batch Info from AP-104 to PC 2 \\
\hline 50.M65 & 11310112 & Adjust AN-106 Feed in AP-104 \\
\hline $0 . \mathrm{M} 70$ & 11310112 & Deliver AN-106 Feed from AP-104 to AP-108 \\
\hline $0 . \mathrm{R} 05$ & 11310112 & Mix SY-101 Feed in AP-102 \\
\hline 150.R10 & 11310114 & Obtain SY-101 Feed Qual Samples from AP-102 \\
\hline $50 . \mathrm{R} 15$ & 11310114 & Provide SY-101 Samples from AP-102 to PC 1 \\
\hline $50 . R 20$ & 11310114 & Analy SY-101 Smpls/Interpret Results from AP-102 \\
\hline $50 . R 25$ & 11310114 & Prov SY-101 Feed Batch Info from AP-102 to PC 1 \\
\hline $50 . \mathrm{R} 30$ & 11310112 & Adjust SY-101 Feed in AP-102 \\
\hline 0.835 & 11310112 & Deliver SY-101 Feed from AP-102 to AP-106 \\
\hline 50.R40 & 11310112 & Mix SY-101 Feed in AP-104 \\
\hline 50.R45 & 11310114 & Obtain SY-101 Feed Qual Samples from AP-104 \\
\hline $50 . \mathrm{R} 50$ & 11310114 & Provide SY-101 Samples from AP-104 to PC 2 \\
\hline 150.R55 & 11310114 & Analy SY-101 Smpls/Interpret Results from AP-104 \\
\hline $0 . R 60$ & 11310114 & Prov SY-101 Feed Batch Info from AP-104 to PC 2 \\
\hline 50.R65 & 11310112 & Adjust SY-101 Feed in AP=104 \\
\hline 150.R70 & 11310112 & Deliver SY-101 Feed from AP-104 to AP-108 \\
\hline 50.705 & 11310112 & Mix SY-103 Feed in AP-102 \\
\hline $150 . T 10$ & 11310114 & Obtain SY-I03 Feed Qual Samples from AP-102 \\
\hline $150 . \mathrm{T} 15$ & 11310114 & Provide SY-103 Samples from AP-102 to PC 1 \\
\hline 150.T20 & 11310114 & Analy SY-103 Smpls/Interpret Results from AP-102 \\
\hline $150 . \mathrm{T} 25$ & 11310114 & Prov SY-103 Feed Batch Info from AP-102 to PC I \\
\hline $150 . T 30$ & 11310112 & Adjust SY-103 Feed in AP-102 \\
\hline $150 . \mathrm{T} 35$ & 11310112 & Deliver SY-103 Feed from AP-102 to AP-106 \\
\hline $150 . \mathrm{T} 40$ & 11310112 & Mix SY-103 Feed in AP-104. \\
\hline $150 . \mathrm{T} 45$ & 11310114 & Obtain SY-103 Feed Qual Samples from AP-104 \\
\hline $150 . T 50$ & 11310114 & Provide SY-103 Samples from AP-104 to PC 2 \\
\hline $150 . \mathrm{T} 55$ & 11310114 & Analy SYY-103 Smpls/Interpret Results from AP-104 \\
\hline $150 . \mathrm{T} 60$ & 11310114 & Prov SY-103 Feed Batch Info from AP-104 to PC 2 \\
\hline $150 . T 65$ & 11310112 & Adjust SY-103 Feed in AP-104 \\
\hline 150.T70 & 11310112 & Deliver SY-103 Feed from AP-104 to AP-108 \\
\hline 150.V05 & 11310112 & Mix AP-101 Feed in AP-102 \\
\hline $150 . \mathrm{V} 10$ & 11310114 & Obtain AP-101 Feed Qual Samples from AP-102 \\
\hline 150.V15 & 11310114 & Provide AP-101 Samples from AP-102 to PC 1 \\
\hline $150 . \mathrm{V} 20$ & 11310114 & Analy AP-101 Smpls/nterpret Results from AP-102 \\
\hline $150 . \mathrm{V} 25$ & 11310114 & Provide AP-101 Feed Batch Info to PC 1 \\
\hline $150 . V 30$ & 11310112 & Adjust AP-101 Feed in AP-102 \\
\hline
\end{tabular}


HNF-1946 Rev. 0

Table 1 Crosswalk from the Level 1 Logic to the Work Breakdown Structure Level 1 Logic Level 6 WBS Description

\begin{tabular}{|c|c|c|}
\hline $150 . V 35$ & 11310112 & Deliver AP-101 Feed from AP-102 to AP-106 \\
\hline $150 . V 40$ & 11310112 & Mix AP-101 Feed in AP-104 \\
\hline $150 . V 45$ & 11310114 & Obtain AP-101 Feed Qual Samples from AP-104 \\
\hline $150 . V 50$ & 11310114 & Provide AP-101 Samples from Ap-104 to PC 2 \\
\hline $50 . \mathrm{V} 55$ & 11310114 & Analy AP-101 Smpls/Interpret Results from AP-104 \\
\hline 50.V60 & 11310114 & Prov AP-101 Feed Batch Info From Ap-104 to PC 2 \\
\hline 50:V65 & 11310112 & Adjust AP-101 Feed in AP-104. \\
\hline $150 . V 70$ & 11310112 & Deliver AP-101 Feed from AP-104 to AP-108 \\
\hline 160.A00 & 11350102 & Final ICD for HLW Feed (ID 20) \\
\hline 160.A02 & 11350101 & Contract and ICD \\
\hline 160.A12 & 11310102 & Estab Tech (Process) Reqmnts-Phase I SIdg Pret \\
\hline 160.A13 & 11310101 & Prepare Plan/Document for Mgmt Assessment (HLW) \\
\hline 160.A15 & 11310102 & Assess Sec Waste, Util Samp Anly Agst Plan (HLW) \\
\hline 160.A16 & 11310102 & Prep DQO for Privatization Char of Pot HLW Feed \\
\hline 60.A17 & 11310102 & Compare RAM/O\&M Concept w/ OTP Results (HLW \\
\hline 160.A 18 & 11310101 & TWRS Management Assessment (HLW) \\
\hline 160.A19 & 11310102 & Retr. Revise Pretreat Rqmts (AZ-101/2 \& AY-102) \\
\hline $160 . \mathrm{A} 20$ & 11310102 & Finalize HLW Pretreat Rqmts $(A Z-101 / 2 \& A Y-102)$ \\
\hline 160.A22 & 11310102 & Determine Appropriate Alternative Tanks \\
\hline 160.A25 & 11310114 & Update BBI \& TCR to Reflect Process Test AZ-101 \\
\hline 160.A26 & 11310103 & Devel Tech Rqmts \& CDR For Addl Eqpt in AZ-101 \\
\hline 160.A32 & 11310110 & Redesign Addl Eqpt Req'd for Sludge Wash-AZ-101 \\
\hline 160.A36 & 11310110 & Construct Equip Req'd for Sludge Washing-AZ-101 \\
\hline 160.A38 & 11310110 & Upgrade AZ-101 W-xxx \\
\hline 160.A40 & 11310110 & Contractor Independent ORR for AZ-10 1 \\
\hline 160.A41. & 11310110 & Develop Draft Sludge Wash Temp Control Rqmts \\
\hline $160 . \mathrm{A} 42$ & 11310110 & DOE ORR for AZ-101 \\
\hline 160.A43 & 11310102 & Optimize PH1 Feed Sludge Wash by Proc Simulation \\
\hline 160.A44 & 11310102 & Deter if Existing Data Meets DQO Needs - AZ-101 \\
\hline 160.A46 & 11310102 & Take Additional Grab Samples from AZ-101 . . \\
\hline $160 . \mathrm{A} 48$ & 11310102 & Perf/Doc Smpl Analysis/Sldg Wash Test-AZ-101 \\
\hline 160.A50 & 11310102 & Verify that AZ-101 is Acceptable for Feed to PC \\
\hline 160.A52 & 11310112 & Pretreat AZ-101 (In-Tank Sludge Washing) \\
\hline $160 . A 62$ & 11310112 & Perf Final Decant/Add Transfer Solution - AZ-101 \\
\hline 160.A64 & 11310112 & Mix Waste to Resuspend Solids for AZ-101 \\
\hline 160.A66 & 11310114 & Obtain HLW Feed Qual Grab Samples AZ-101 Solids \\
\hline 160.A68 & 11310114 & Eval Samples/Establish MT for AZ-101 \\
\hline 160.A70 & 11360104 & PC Conduct Waste Form Qualification - AZ-101 \\
\hline 160.A72 & 11310112 & Deliver Second Batch of Feed from AZ-101 \\
\hline
\end{tabular}


HNF-1946 Rev. 0

Table 1 Crosswalk from the Level 1 Logic to the Work Breakdown Structure Level 1 Logic Level 6 WBS

Description

\begin{tabular}{|c|c|c|}
\hline 160.A74 & 11310114 & Provide Separate Samples to the PC - AZ-101 \\
\hline 160.A76 & 11310114 & Prov MT Non-Volatile Oxides Exclude Na/Si-AZ-101 \\
\hline 160.A78 & 11310112 & Deliver First Batch of Feed from AZ-101 \\
\hline 160.F00 & 11310102 & Deter if Existing Data Meets DQO Needs - AZ-102 \\
\hline 160.F05 & 11310102 & Obtain Core Smpls/Prep Subsmpls/Comp for AZ-102 \\
\hline 160.F10 & 11310102 & Perf/Doc Smpl Análysis/SldgWash Testing - AZ-102 \\
\hline 160.F12 & 11310102 & Update BBI and TCR for AZ-102 \\
\hline 160.F15 & 11310102 & Confirm that AZ-102 is Acceptable for Feed to PC \\
\hline 160.F30 & 11310103 & Revise W-211 FDC for $\mathrm{AZ}-102$ \\
\hline 160.F50 & 11310114 & Obtain HLW Feed Qual Grab Samples AZ-102 Solids \\
\hline 160.F55 & 11310114 & Eval Smpls/Estab MTof Non-Volatile for AZ-102 \\
\hline 160.F65 & 11310112 & Pretreat AZ-102 (In-Tank Sludge Washing) \\
\hline $160 . F 70$ & 11310112 & Perf Final Decant/Add Transfer Solution - AZ-102 \\
\hline 160.F75 & 11310112 & Mix Waste to Resuspend Solids for AZ-102 \\
\hline 160.F80 & 11360102 & PC Conduct Waste Form Qual Confirmation-AZ-102 \\
\hline 160.F85 & 11310114 & Provide Separate Samples to Private Contractor \\
\hline 160.F90 & 11310114 & Provide MT Non-Volatile Oxides Excluding $\mathrm{Na} / \mathrm{Si}$ \\
\hline 160.F95 & 11310112 & Deliver First Batch of Feed from AZ-102 \\
\hline 160.F99 & 11310112 & Deliver Second Batch of Feed from AZ-102 \\
\hline 160.HOO & 11310102 & Deter if Existing Data Meets DQO Needs - AY-102 \\
\hline 160.H05 & 11310102 & Obtn Core Smpls/Prep Subsmpls/Comp for AY-102 \\
\hline 160.H17 & 11310103 & Revise W-211 FDC for AY-102 \\
\hline $160 . \mathrm{H} 20$ & 11310102 & Pexf/Doc Smpl Analyșis/Sldg Wașh Testing-AY-102 \\
\hline $160 . \mathrm{H} 22$ & 11310102 & Update BBI and TCR for $\mathrm{AY}-102$ \\
\hline $160 . \mathrm{H} 25$ & 11310114 & Confirm that $\mathrm{AY}-102$ is Acceptable for Feed to PC \\
\hline $160 . \mathrm{H} 27$ & 11310112 & Pretreat AY-102 (In-Tank Sludge Washing). \\
\hline 160.H45 & 11310112 & Perf Final Decant/Add Transfer Solution-AY-102 \\
\hline $160 . \mathrm{H} 47$ & 11310112 . & Mix .Waste to Resuspend Solids for AY-102 \\
\hline $160 . \mathrm{H} 50$ & 11310114 & Obtain HLW Feed Qual Grab Samples AY-102 Solids \\
\hline 160.1455 & 11310114 & Eval Smpls/Estab MT of Non-Volatile for AY-102 \\
\hline $160 . \mathrm{H} 60$ & 113601.14 & PC Conduct Waste Form Qual/Confirmation-AY-102 \\
\hline 160.H65 & 11310114 & Provide MT Non-Volatile Oxides Excluding $\mathrm{Na} / \mathrm{Si}$ \\
\hline 160.H70 & 11310114 & Provide Separate Samples to PC - AY-102 \\
\hline $160 . \mathrm{H} 75$ & 11310112 & Deliver First Batch of Feed from AY-102 \\
\hline $160 . \mathrm{H} 80$ & 11310112 & Deliver Second Batch of Feed from AY-102 \\
\hline 160.H85 & 11310112 & Deliver Third Batch of Feed from AY -102 \\
\hline 160.H் 90 & 11310112 & Deliver Fourth Batch of Feed from AY-102 \\
\hline $160 . \mathrm{H} 95$ & 11310112 & Deliver Fifth Batch of Feed from AY-102 \\
\hline 160.300 & 11310102 & Deter if Existing Data Meets DQO Needs - C-104 \\
\hline
\end{tabular}


HNF-1946 Rev. 0

Table 1 Crosswalk from the Level 1 Logic to the Work Breakdown Structure

Description

\begin{tabular}{|c|c|c|}
\hline 160.J05 & 11310102 & Obtain Core Smpls/Prep Subsmpls/Comp for C-104 \\
\hline $160 . \mathrm{J} 10$ & 11310102 & Perf/Doc Smpl Analysis/SldgWash Testing C-104 \\
\hline $160 . J 12$ & 11310102 & Update BBI and TCR for C-104 \\
\hline 160.J15 & 11310102 & Confirm that C-104 is Acceptable for Feed to PC \\
\hline $160 . \mathrm{J} 50$ & 11310114 & Obtain HLW Feed Qual Grab Samp C-104/AZ-101 \\
\hline $160 . J 55$ & 11310114 & Eval Smpls/Estab MT of Non-Volatile for C-104 \\
\hline 160.365 & 11310112 & Pretreat C-104/AZ-101 (In-Tank Sludge Washing) \\
\hline 160.J70 & 11310112 & Perf Decant/Add Transfer Solution - C-104/AZ-101 \\
\hline 160.375 & 11310112 & Mix Waste to Resuspend Solids for C-104/AZ-101 \\
\hline $160 . J 80$ & 11310102 & PC Conduct Waste Form Qual Confirm -C-104/AZ-101 \\
\hline $160 . J 85$ & 11310114 & Provide Separate Samples to Private Contractor \\
\hline 160.590 & 11310114 & Provide MT Non-Volatile Oxides Excluding Na/Si \\
\hline 160.J95 & 11310112 & Deliver lst Batch of Feed from C-104/AZ-101 \\
\hline 160.197 & 11310112 & Deliver 2nd Batch of Feed from C-104/AZ-101 \\
\hline 160.399 & 11310112 & Deliver 3rd Batch of Feed from C-104/AZ-101 \\
\hline 250.110 & 11310126 & Project W-314 (Phase I) Management \\
\hline 250.120 & 11310126 & Project W-314 (Phase I) Permits/Safety Analysis \\
\hline 250.130 & 11310140 & Project W-314 (Phase I) Implementation \\
\hline $250.4 \mathrm{~A} 0$ & 11310126 & W-314 Master Pump Shut Down Upgrades \\
\hline $250.4 \mathrm{C} 0$ & 11310126 & W-314 Upgrades to AN Farm \\
\hline $250.4 \mathrm{D} 0$ & 11310126 & W-314 AZ Farm Upgrades \\
\hline $250.4 \mathrm{E} 0$ & 11310126 & W-314 200 E/W Piping Upgrades \\
\hline $250.4 \mathrm{E} 5$ & 11310126 & Prepare AW Farm for Construction Actitivies \\
\hline $250.4 \mathrm{FO}$ & 11310126 & W-314 AW Farm Upgrades \\
\hline $250.4 \mathrm{G} 0$ & 11310126 & W-314 Upgrades to AY Farm \\
\hline $250 . \mathrm{Col}$ & 11310126 & Prepare AN Tank Farm for W-314 Construction \\
\hline $250 . \mathrm{T} 01$ & 11360126 & M-43-12 Start Const for Upgrade in 1st Farm \\
\hline 250.T011 & 11360126 & M-43-11 Provide W-314 Const Sched to Ecology \\
\hline $250 . \mathrm{T} 013$ & 11360126 & M-43-13 Start Const for Upgrade in 2nd Farm \\
\hline $250 . \mathrm{T} 014$ & 11360126 & M-43-14 Start Const for Upgrade in 3rd Farm \\
\hline $250 . \mathrm{T} 015$ & 11360126 & M-43-15 Start Const for Upgrade in 4th Farm \\
\hline 250.TA & 11360126 & W-314 Complete Phase 1 \\
\hline 270.599 & 11310103 & ISSTRS Prj 1st Tank C-104 \& Retrieve to AN-105 \\
\hline 270.J99 & 11310107 & ISSTRS Prj 1st Tank C-104 \& Retrieve to AN-105 \\
\hline 270.399 & 11310109 & ISSTRS Prj 1st Tank C-104 \& Retrieve to AN-105 \\
\hline 330.010 & 11380101 & Project Administration/Management/Engineering \\
\hline 330.010 & 11380103 & Project Administration/Management/Engineering \\
\hline 330.010 & 1138,0104 & Project Administration/Management/Engineering \\
\hline 330.020 & 11380102 & Systems Definition \\
\hline
\end{tabular}


HNF-1946 Rev. 0

Table 1 Crosswalk from the Level 1 Logic to the Work Breakdown Structure Level 1 Logic Level 6 WBS

Description

\begin{tabular}{|c|c|c|}
\hline 330.020 & 11380103 & Systems Definition \\
\hline 330.030 & 11380103 & IC Document Revisions/IC Drawings \\
\hline 330.040 & 11380103 & Compl Infrastructure Electrical Sys Contract Pkg \\
\hline 330.050 & 11380103 & Design/Construction/Startup Electrical System \\
\hline 330.060 & 11380103 & Dsn/Construction Site Sys for PC Construction \\
\hline 330.070 & 11380103 & Dsn/Construct/S/up Water, Site, Liq Eff Systems \\
\hline 330.080 & 11380104 & Maint/Op/Provide Vendor Utils for Constr PC PhI \\
\hline 330.080 & 11380105 & Maint/Op/Provide Vendor Utils for Constr $\mathrm{PC} \mathrm{PhI}$ \\
\hline 330.090 & 11380104 & Maint/Op/Prov Vendor Utils/Wst Disp S/up PC PhI \\
\hline 330.090 & 11380105 & Maint/Op/Prov Vendor Utils/Wst Disp S/up PC PhI \\
\hline $330: 100$ & 11380104 & Maint/Op/Prov Vendor/Wst Disp Prodctn PC PhI \\
\hline 330.100 & 11380105 & Maint/Op/Prov Vendor/Wst Disp Prodctn PC PhI \\
\hline 330.120 & 11380104 & Provide Vendor Staff Training \\
\hline 350.015 & 11360101 & PC Complete Phase 1A Deliverables \\
\hline 350.040 & 11360102 & Modify AP-106 As Needed/Tie-In - PC 1 \\
\hline 350.090 & 11360103 & PC 1 Processes AN-105 Feed from AP-106 \\
\hline 350.100 & 11360103 . & PC 1 Processes AN-104 Feed from AP-106 \\
\hline 350.110 & 11360103 & PC 1 Processes AW-101 Feed from AP-106 \\
\hline 350.120 & 11360103 & PC 1 Processes AN-103 Feed from AP-106 \\
\hline 350.123 & 11360103 & PC 1 Processes AP-101 Feed from AP-106 \\
\hline 350.125 & 11360103 & PC 1 Processes AY-101 Feed from AP-106 \\
\hline 350.130 & 11360103 & PC 1 Processes AN-107 Feed from AP-106 \\
\hline 350.135 & 11360103 & Contract \#1 Process 7th Feed Batch \\
\hline 350.140 & 11360103 & PC 1 Processes AN-102 Feed from AP-106 \\
\hline 350.150 & 11360103 & PC 1 Processes AN-106 Feed from AP-106 \\
\hline 350.160 & 11360103 & PC 1 Processes SY-101 Feed from AP-106 \\
\hline 350.170 & 11360103 & PC 1 Processes SY-103 Feed from AP-106 \\
\hline 350.230 & 11360102 & Modify AP-108 As Needed/Tie-In - PC 2 \\
\hline 350.239 & 11360102 & M-60-12 Start Hot Ops of LLW Pretreat Facility \\
\hline 350.240 & 11360103 & PC 2 Processes AN-105 Feed from AP-108 \\
\hline 350.250 & 11360103 & PC 2 Processes AN-104 Feed from AP-108 \\
\hline 350.260 & 11360103 & PC 2 Processes AW-101 Feed from AP-108 \\
\hline 350.270 & 11360103 & PC 2 Processes AN-103 Feed from AP-108 \\
\hline 350.273 & 11360103 & PC 2 Processes AW-104 Feed from AP-108 \\
\hline 350.275 & 11360103 & PC 2 Processes AY-101 Feed from AP-108 \\
\hline 350.280 & 11360103 & PC 2 Processes AN-107 Feed from AP-108 \\
\hline 350.285 & 11360103 & Contractor \#1 Process 7th Feed Batch \\
\hline 350.290 & 11360103 & PC 2 Processes AN-102 Feed from AP-108 \\
\hline 350.300 & 11360103 & PC 2 Processes AN-106 Feed from AP-108 . \\
\hline
\end{tabular}


HNF-1946 Rev. 0

Table 1 Crosswalk from the Level 1 Logic to the Work Breakdown Structure

Level 1 Logic Level 6 WBS

Description

\begin{tabular}{|c|c|c|}
\hline 350.310 & 11360103 & PC 2 Processes SY-101 Feed from AP-108 \\
\hline 350.320 & 11360103 & PC 2 Processes SY-103 Feed from AP-108 \\
\hline $350 . .05$ & 11310112 & Transfer PC\#1 \& PC\#2 Solids to AP-107 \\
\hline 360.020 & 11360102 & Final Design HLW/LAW Immobilization Facility \\
\hline 360.040 & 11360102 & Tie-In Feed Line to Valve Pit Provided by W-211 . \\
\hline 360.080 & $113601 \dot{0} 4$ & Phase 1B Proc/Immb First Batch HLW Feed-AZ-10i \\
\hline 360.090 & 11360104 & Phase 1B Proc/Immb Second Batch HLW Feed-AZ-101 \\
\hline 360.100 & 11360104 & Phase 1B Proc/Immob Third Batch HLW Feed-AZ-102 \\
\hline 360.110 & 11360104 & Phase IB Proc/Immob Fourth Batch HLW Feed-AZ-102 \\
\hline 360.120 & 11360104 & Phase 1B Proc/Immb Fifth Batch HLW Feed-AZ-102 \\
\hline 360.130 & 11360104 & Phase 1B Proc//mmb Sixth Batch HLW Feed-AZ-102 \\
\hline 360.140 & 11360104 & Phase 1B Proc/Immb Seventh Batch HLW Feed-AY-102 \\
\hline 360.150 & 11360104 & Phase 1B Proc/Immb Eighth Batch HLW Feed-AY-102 \\
\hline 360.160 & 11360104 & Phase IB Proc/Immb Ninth Batch HLW Feed-AY-102 \\
\hline 360.170 & 11360104 & Phase 1B Proc/Immob Tenth Btch of HLW Feed C-104 \\
\hline 360.180 & 11360104 & Phase 1B Proc/Immob Eleventh Batch of HLW C-104 \\
\hline 360.190 & 11360104 & Phase 1B Proc/Immob Twelveth Batch of HLW C-104 \\
\hline 440.010 & 11340201 & IHLW Interim Storage Administration/Management \\
\hline 440.010 & 11340202 & IHLW Interim Storage Administration/Managẹment \\
\hline 440.020 & 11340202 & Prepare/Maintain ICDs (ID 13,14,17) \\
\hline 440.030 & 11340202 & Project Management Plans \\
\hline 440.040 & 11340202 & Maintain Technical Requirements (DRD) \\
\hline 440.050 & 11340203 & IHLW Storage Conceptual Design (W-464) \\
\hline 440.060 & 11340203 & Advanced Conceptual Design (W-464) \\
\hline 440.070 & 11340203 & Project Validation (W-464) \\
\hline 440.090 & 11340203 & Procure Equipment (W-464) \\
\hline 440.100 & 11340203 & Design IHLW Storage Facility (W-464) \\
\hline 440.110 & 11340104 & Prepare NEPA Documentation (W-464) \\
\hline 440.110 & 11340203 & Prepare NEPA Documentation (W-464) \\
\hline 440.115 & 11340203 & Prepare RCRA Permits (W-464) \\
\hline $440.115 \mathrm{~A}$ & 11340203 & M-20-56 Submit Canister Fac Part B Permit App \\
\hline $440.115 \mathrm{C}$ & 11340203 & M-90-12: Sub Revised CS Facility Part A Permit \\
\hline 440.120 & 11340104 & Authorization Basis Development \& Appr (W-464) \\
\hline 440.120 & 11340203 & Authorization Basis Development \& Appr (W-464) \\
\hline 440.140 & 11340203 & Modify/Construct System (W-464) \\
\hline 450.010 & 11340203 & Prep OTPs/Conduct Readiness Assessmnt (Phase I) \\
\hline $450.010 \mathrm{D}$ & 11340203 & M-90-11: Complete Startup-Phase I-HLW ISF (CSB) \\
\hline 450.030 & 11340204 & Transpoit/Receive/Interim Store IHLW (Phase I) \\
\hline 460.005 & 11340101 & ILAW Disposal Administration/Management \\
\hline
\end{tabular}


HNF-1946 Rev. 0

Table 1 Crosswalk from the Level 1 Logic to the Work Breakdown Structure Level 1 Logic Level 6 WBS

Description

\begin{tabular}{|c|c|c|}
\hline 460.005 & 11340102 & ILAW Disposal Administration/Management \\
\hline $460.005 \mathrm{~A}$ & 11340203 & Isss Ltr Rpt on SE Eval Processes DNFSB 92-4 \#2 \\
\hline 460.015 & 11340102 & Maintain ICDs \\
\hline 460.015 & 11340104 & Maintain ICDs \\
\hline 460.030 & 11340102 & Maintain Technical Requirements (DRD) \\
\hline 460.035 & 11340102 & Project Management Plan \\
\hline $460.035 \mathrm{~A}$ & 11340102 & M-90-01 Submit Project Management Plans to Ecol \\
\hline 460.040 & 11340104 & ILAW Storage Conceptual Design (W-465) \\
\hline $460.040 \mathrm{E}$ & 11340104 & M-90-02-T01: Comp Conceptual Design-ILAW ISF \\
\hline 460.045 & 11340104 & ILAW Storage Adv Conceptual Design (W-465) \\
\hline 460.060 & 11340104 & Project Validation (W-465) \\
\hline 460.065 & 11340104 & ILAW Storage Detailed Design (W-465) \\
\hline $460.065 \mathrm{~K}$ & 11340104 & M-90-02-T01: Complete Detailed Design-ILAW ISF \\
\hline 460.070 & 11340104 & Modify Vaults (W-465) \\
\hline $460.070 \mathrm{H}$ & 11340104 & M-90-03: CD 3-Initiate Construction - ILAW ISF \\
\hline 460.075 & 11340102 & Prepare NEPA Documentation (W-465) \\
\hline 460.075 & 11340104 & Prepare NEPA Documentation (W-465) \\
\hline 460.080 & 11340104 & Prepare RCRA Permits (W-465) \\
\hline $460.080 \mathrm{D}$ & 11340104 & M-20-57: Sub ILAW Part B Permit App to Ecology \\
\hline 460.085 & 11340104 & Authorization Basis Development \& Appr (W-465) \\
\hline 460.095 & 11340102 & Prepare/Maintain DRD - Disposal Facilities \\
\hline 460.105 & 11340106 & Conceptual Design - Disposal Facilities (W-520) \\
\hline $460.105 \mathrm{H}$ & 11340106 & M-90-07-T01: Comp Conceptual Dsn-ILAW Add'1 S\&D \\
\hline 460.110 & 11340106 & Advanced Conceptual Dsn - Disposal Fac (W-520) \\
\hline 460.112 & 11340106 & Validate Project $(W-520)$ \\
\hline 460.115 & 11340106 & Design Disposal Facilities (W-520) \\
\hline $460.115 \mathrm{I}$ & 11340106 & M-90-09-T01: Comp Detailed Dsn-ILAW Add'1 S\&D \\
\hline $460: 120$ & 11340106 & Construct Disposal Facilities (W-520) \\
\hline $460.120 \mathrm{C}$ & 11340106 & M-90-08: CD 3-Init Construction-ILAW Add'l S\&D \\
\hline 460.125 & 11340106 & Regulatory Requirements for Disposal Facilities \\
\hline $460.125 \mathrm{~A}$ & 11340106 & M-20-58 Submit LAW Facility Part B Permit \\
\hline 460.130 & 11340106 & Authorization Basis Development and Approval \\
\hline 460.140 & $1134013 \mathrm{E}$ & 1998 Performance Assessment \\
\hline 460.145 & $1134013 B$ & Data Collection for 2001 Performance Assessment \\
\hline $460.145^{\circ}$ & $1134013 \mathrm{E}$ & Data Collection for 2001 Performance Assessment \\
\hline 460.145 & $1134013 \mathrm{M}$ & Data Collection for 2001 Performance Assessment \\
\hline 460.155 & $1134013 \mathrm{E}$ & 2001 Performance Assessment \\
\hline $460.155 \mathrm{~A}$ & 11340103 & Interface to $\mathrm{H} 101020$ (PMBS 400.020) \\
\hline 460.160 & 11340105 & Data Collection for Performance Assessment \\
\hline
\end{tabular}


HNF-1946 Rev. 0

Table 1 Crosswalk from the Level I Logic to the Work Breakdown Structure Level 1 Logic Level 6 WBS Description

$\begin{array}{lll}460.170 & 11340105 & \text { Maintain ILAW Phase I Performance Assessment } \\ 460.170 \mathrm{~F} & 11340105 & \begin{array}{l}\text { M-90-05-T01: Submit ILAW Disp Fac PA To Ecology } \\ 470.010\end{array} \\ 47340104 & \text { Procedures/Training/OTP/ORR (Initial Phase I) } \\ 470.010 & 11340105 & \text { Procedures/Training/OTP/ORR (Initial Phase I) } \\ 470.040 & 11340105 & \text { Interim Store ILAW Operations (Initial Phase I) } \\ 470.040 \mathrm{~A} & 11340105 & \text { M-90-06: Initiate Hot Ops - ILAW Grout Vaults } \\ 470.060 & 11340105 & \text { Procedures/Training/OTP/ORR (Balance Phase I) } \\ 470.069 \mathrm{~A} & 11340105 & \text { M-90-10 Initiate Hot Ops of ILAW Disposal Fac } \\ 470.075 & 11340105 & \text { Dispose ILAW Operations (Balance Phase I) } \\ 470.080 & 11340107 & \text { Close ILAW Disposal Facilities } \\ 490.090 & 11340202 & \text { Negotiate RW Requirements } \\ 620.015 & 11310102 & \text { Develop Waste Feed Delivery Safety Basis DQO } \\ 620.020 & 11310102 & \text { Maintain Waste Feed Delivery System Summary } \\ 620.030 & 11310113 & \text { Perform Feed Delivery USQ Process for HLW/LLW } \\ 620.050 & 11310113 & \text { Develop Waste Feed Delivery Licensing Strategy } \\ 620.055 & 11310102 & \text { Develop Waste Feed Delivery Permitting DQO } \\ 620.060 & 11310102 & \text { Eval Envmmntl Baseline HLW/LLW Feed Delivery } \\ 620.070 & 11310102 & \text { Update Environmental Baseline } \\ 620.090 & 11310102 & \text { Maintain Environmtl Baseline for Phase I Ext Ops } \\ 620.095 & 11310101 & \text { Dev/Iss Draft WFD Tech Baseline Summary } \\ 620.097 & 11310101 & \text { Update WFD Technical Baseline Summary } \\ & & \end{array}$


Table 1 Crosswalk from the Level 1 Logic to the Work Breakdown Structure Level 6 List of Terms

$\begin{array}{ll}\text { CDR } & \text { Conceptual Design Report } \\ \text { CSB } & \text { Canister Storage Building } \\ \text { DNFSB } & \text { Defense Nuclear Facilities Safety Board } \\ \text { DOE-RC } & \text { U. S. Department of Energy, Richland Operations Office } \\ \text { DQO } & \text { Data Quality Objective } \\ \text { DRD } & \text { design requirement document } \\ \text { DST } & \text { double-shell tank } \\ \text { EIS } & \text { Environmental Impact Statement } \\ \text { FDC } & \text { Functional Design Criteria } \\ \text { FGWL } & \text { flammable gas watch list } \\ \text { ICD } & \text { interface control document } \\ \text { IHLW } & \text { immobilized high-level waste } \\ \text { ILAW } & \text { immobilized low-activity waste } \\ \text { ISF } & \text { interim storage facility } \\ \text { ISSTRS } & \text { Initial Single-Shell Tank Retrieval System } \\ \text { LAW } & \text { low-activity waste } \\ \text { LLW } & \text { low-level waste } \\ \text { LMHC } & \text { Lockheed Martin Hanford Corporation } \\ \text { NEPA } & \text { National Environmental Policy Act of 1969, 42 USC 4321, et seq. } \\ \text { ORR } & \text { operational readiness review } \\ \text { OTP } & \text { operational test plan } \\ \text { PA } & \text { performance assessment } \\ \text { PC } & \text { private contractor } \\ \text { PHMC } & \text { Project Hanford Management Contract } \\ \text { RCRA } & \text { Resource Conservation and Recovery Act of 1976, 42 USC 6901, et seq. } \\ \text { ROD } & \text { Record of Decision } \\ \text { S\&D } & \text { storage and disposal } \\ \text { SST } & \text { single-shell tank } \\ \text { TCR } & \text { Tank Characterization Report } \\ \text { USQ } & \text { unreviewed safety question } \\ \text { WFD } & \text { waste feed delivery } \\ & \text {. }\end{array}$


- Effluent Treatment Facility treatment of 242-A Evaporator condensate through FY 2032

- . Liquid Effluent Retention Facility treatment of radioactive or dangerous liquid effluents generated by private contractors through FY 2032

Treated Effluent Disposal Facility treatment of non-radioactive, nondangerous liquid effluents generated by private contractors through FY 2032.

- $\quad$ Ongoing TWRS Safe Storage work scope for Tank Farm Operations (TW03), Tank Waste Characterization (TW04), and Tank Safety Issue Resolution (TW02). The following work is funded elsewhere in the TWRS and is documented in the TWRS FY 1998 MYWP (Lenseigne 1997):

- Operation and maintenance of the single-shell tank farms through FY 2004 and the double-shell tank farms through FY 2005 including Environmental, Safety and Health training, maintenance, operations, surveillance and engineering

- Tank Farm Upgrade Project W-314 after FY 2000 (Phase 2 of W-314)

- Characterization work scope responding to Defense Nuclear Facilities Safety Board (DNFSB) 93-5, DNFSB Recommendation 93-5 to the Secretary of Energy Regarding Hanford Tank Sampling and Characterization (DNFSB 1993) and Hanford Federal Facility Agreement and Consent Order (Tri-Party Agreement) (Ecology et al. 1996) M-44 commitments; includes Characterization work scope through FY 2001

- Waste Tank Safety work scope planned for resolution of unreviewed safety questions (USQ) surrounding flammable gas and organics through FY 2001.

- Management Support (TW10) to TWRS (excluding the allocated TWRS Retrieval and Disposal business volume share) for Environmental oversight, Systems Engineering, reengineering, program integration, RL support, Quality Assurance, Safety and Health oversight, life-cycle cost estimating, Project Hanford Management Contract fee, etc. This work is funded elsewhere in the TWRS and is documented in the TWRS FY 1998 MYWP (Lenseigne 1997):

- Costs for operation of the private contractors' facilities and payment for immobilized product produced. (Refer to the Privatization Phase 1 boxes in Figure 4, TWRS Retrieval and Disposal WBS.) This work is funded elsewhere in the TWRS and is documented in the TWRS FY 1998 MYWP (Lenseigne 1997). 
HNF-1946 Rev. 0

- The Privatization Regulatory Unit, which is separately funded by the U.S. Department of Energy outside of TWRS.

- $\quad$ Phase 2 costs for the TWRS Retrieval and Disposal Program and private contractors' work. Refer to the gray shaded boxes in Figure 4, TWRS Retrieval and Disposal Work Breakdown Structure. This work is funded elsewhere in the TWRS and is documented in the TWRS FY 1998 MYWP (Lenseigne 1997). 


\subsection{SCHEDULE}

The TWRS schedule has been updated to reflect changes that resulted from the RTP development phase. The schedule products are discussed in the following sections and are included as figures or tables in this section.

\subsection{SUMMARY SCHEDULE}

The TWRS Retrieval and Disposal Summary Schedule for the Phase $1 \mathrm{~B}$ mission is illustrated in Figure 6 . The major programmatic start and end points to support the private contractors are depicted.

\subsection{SITE MASTER BASELINE SCHEDULE}

The Site Master Baseline Schedule is illustrated in Figure 7. This schedule is an initial update to reflect the integrated RTP planning effort for Phase 1B of the TWRS Retrieval and Disposal Program. This schedule has been summarized from the lower level detail, includes critical path, and is traceable to the Program Logic (Figure 3).

\subsection{PROJECT MASTER BASELINE SCHEDULE}

The Project Master Baseline Schedule is illustrated in Figure 8. This schedule is an initial update to reflect the integrated RTP planning effort for Phase 1B of TWRS Retrieval and Disposal Program. This schedule has been summarized from the lower level detail, includes each Level 1 Logic activity grouped by Level 6 WBS element, and shows critical path. This schedule is consistent with the Level 1 Logics and TBR packages and is supported by the lower level logic network.

\subsection{MILESTONE COMPARISON}

Table 2 provides a comparison of RL, Headquarters, and Tri-Party Agreement milestones between the baseline contained in the TWRS FY 1998 MYWP (Lenseigne 1997), the Tri-Party Agreement, and the revised RTP planning for Phase 1B of the TWRS Retrieval and Disposal Program. 
HNF-1946 Rev. 0

This page intentionally left blank. 
Figure 6. Summary Schedule.

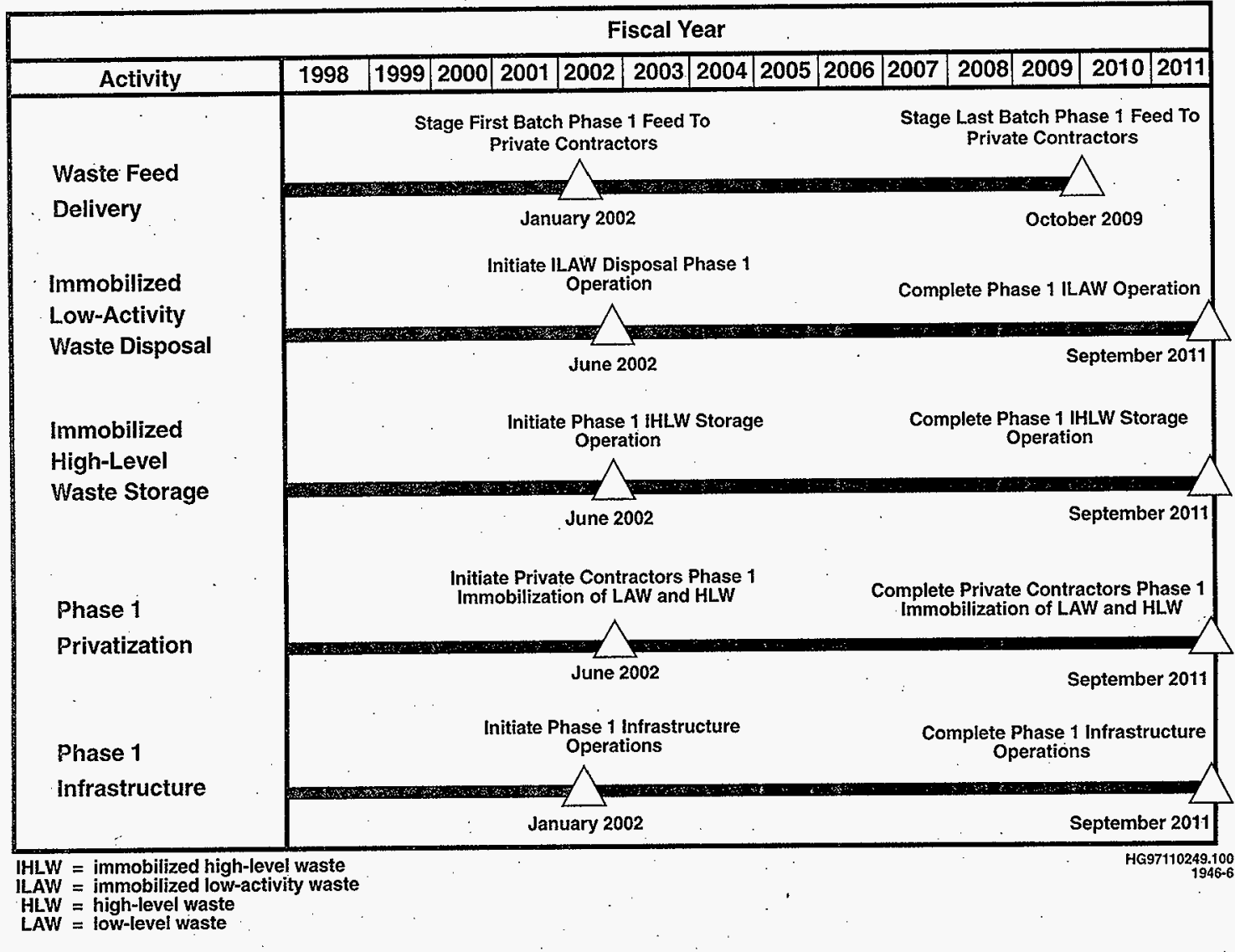


HNF-1946 Rev. 0

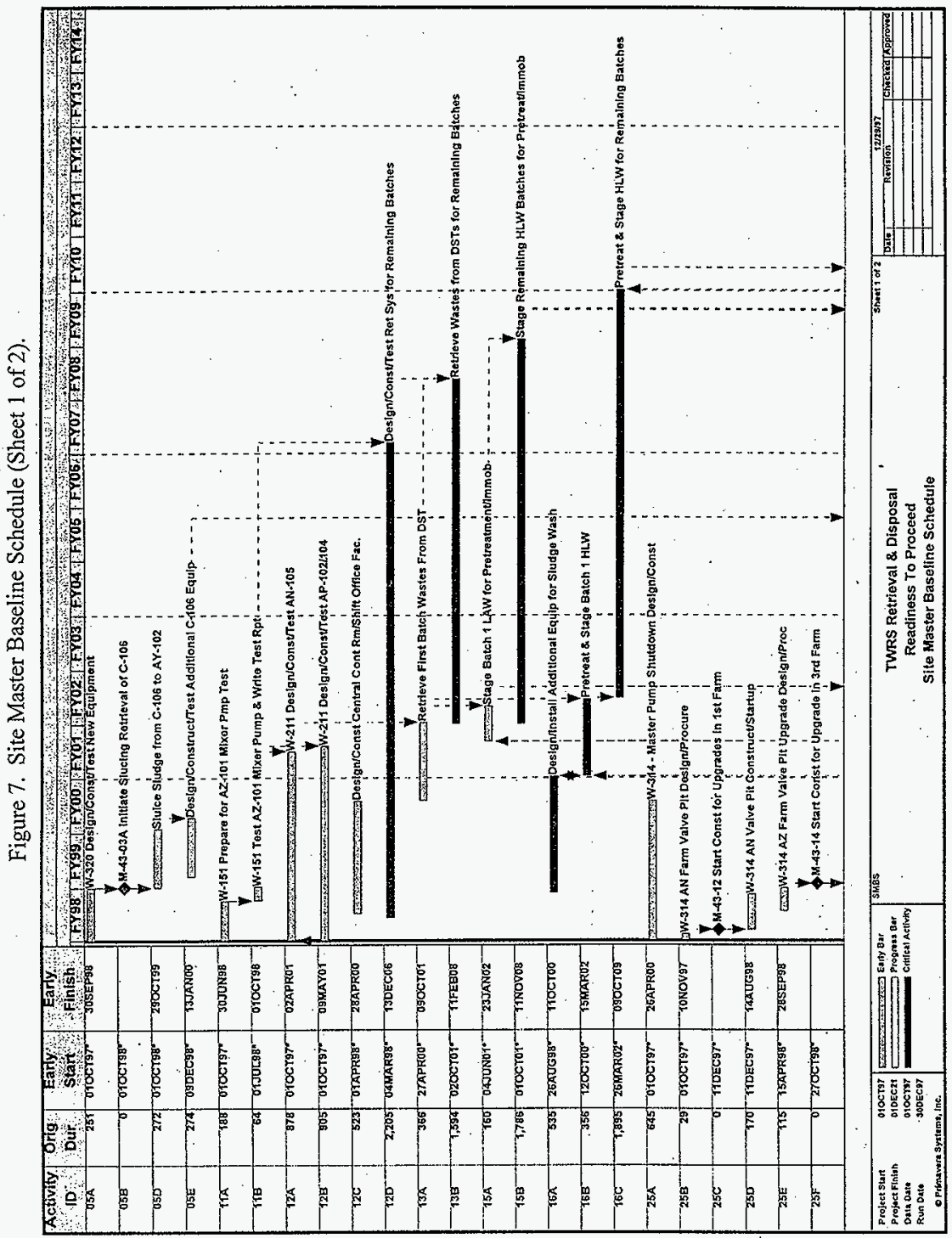


HNF-1946 Rev. 0

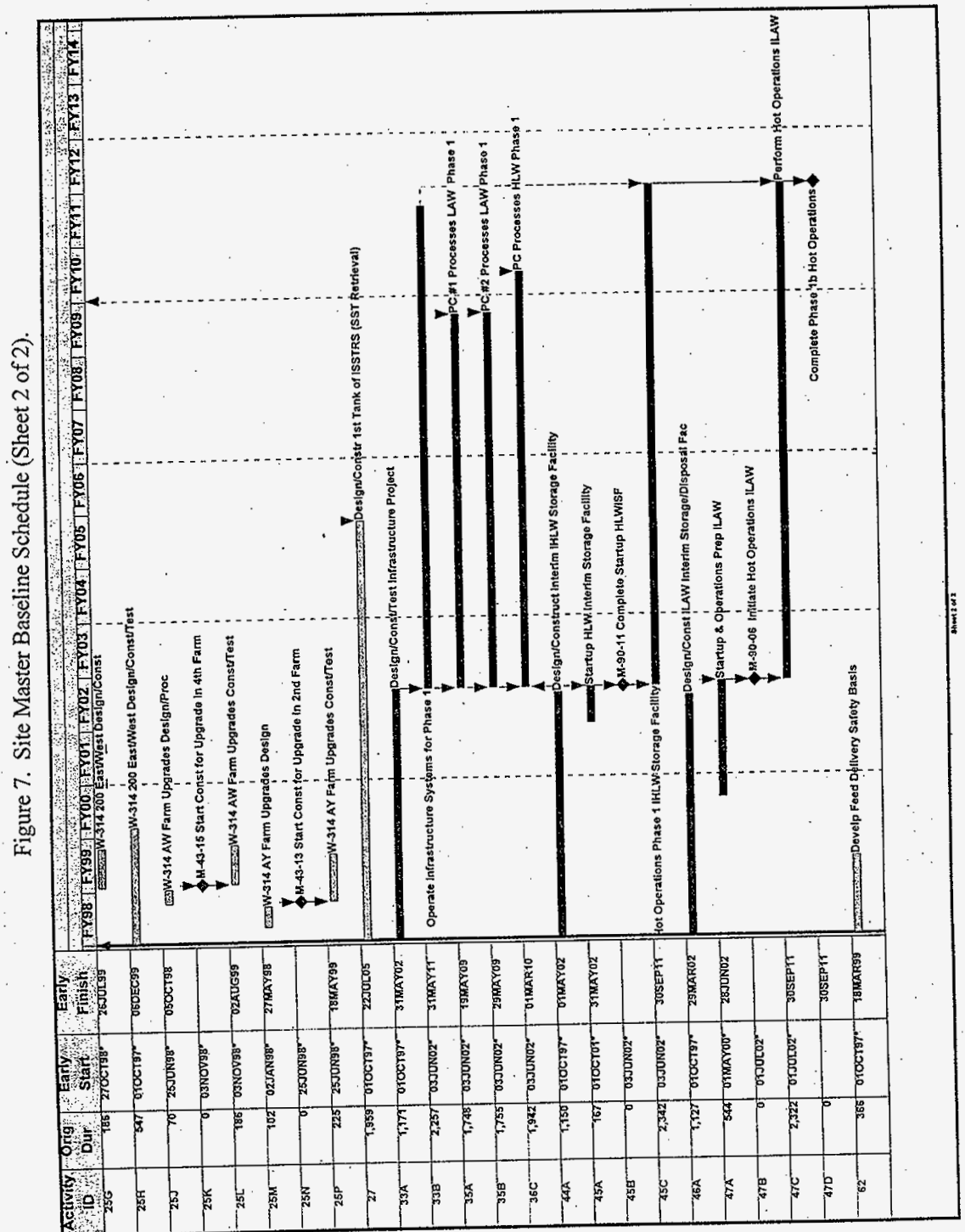




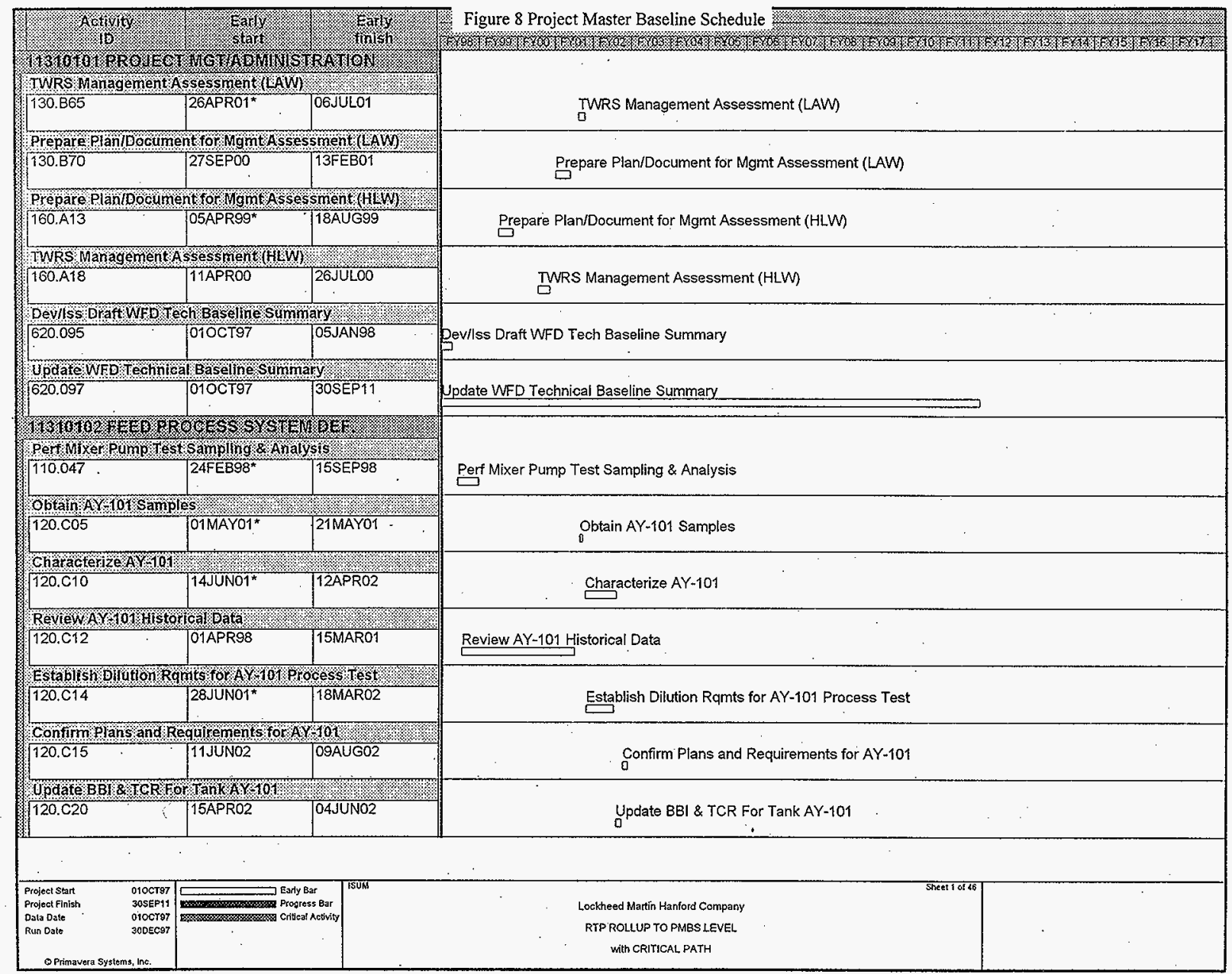




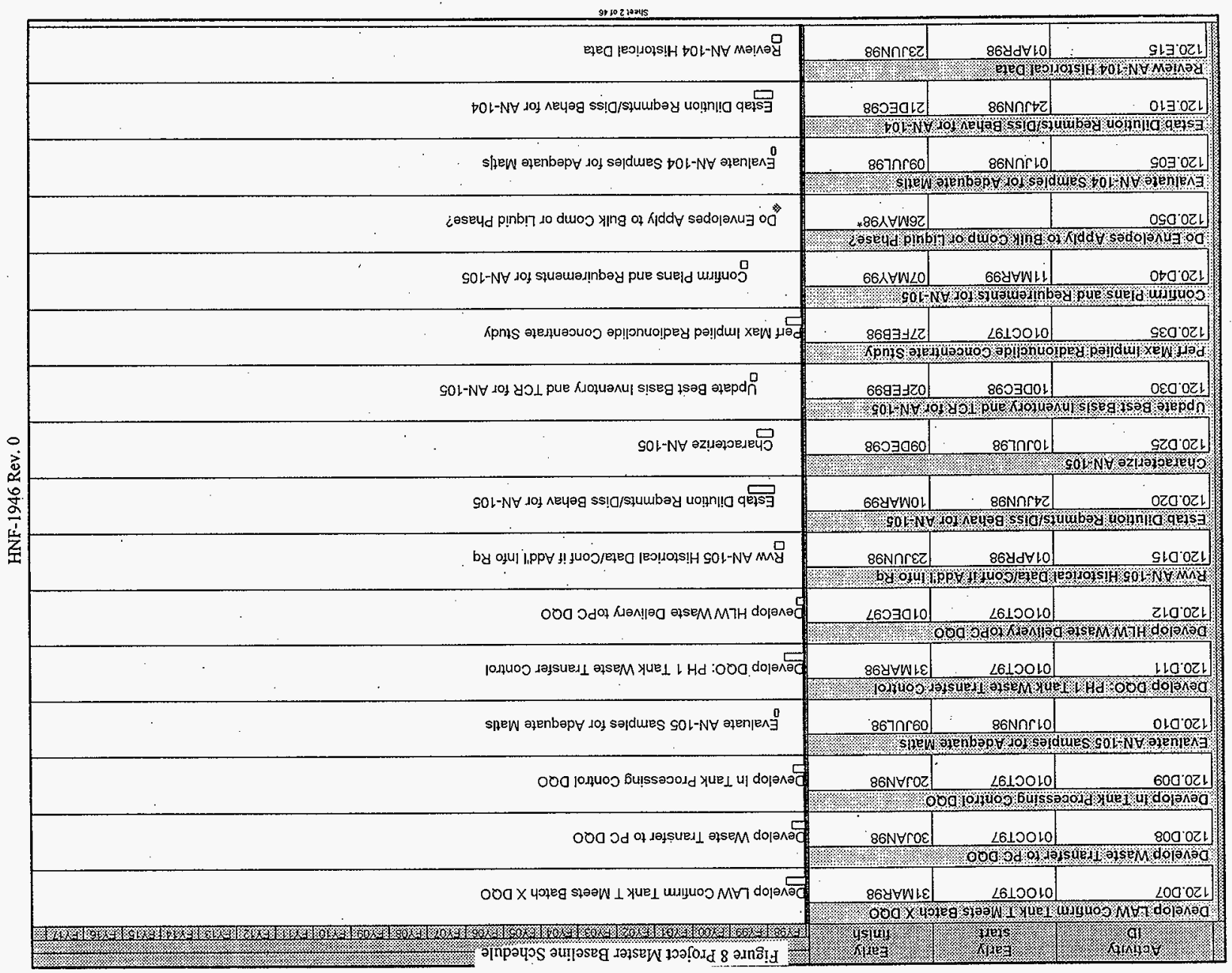




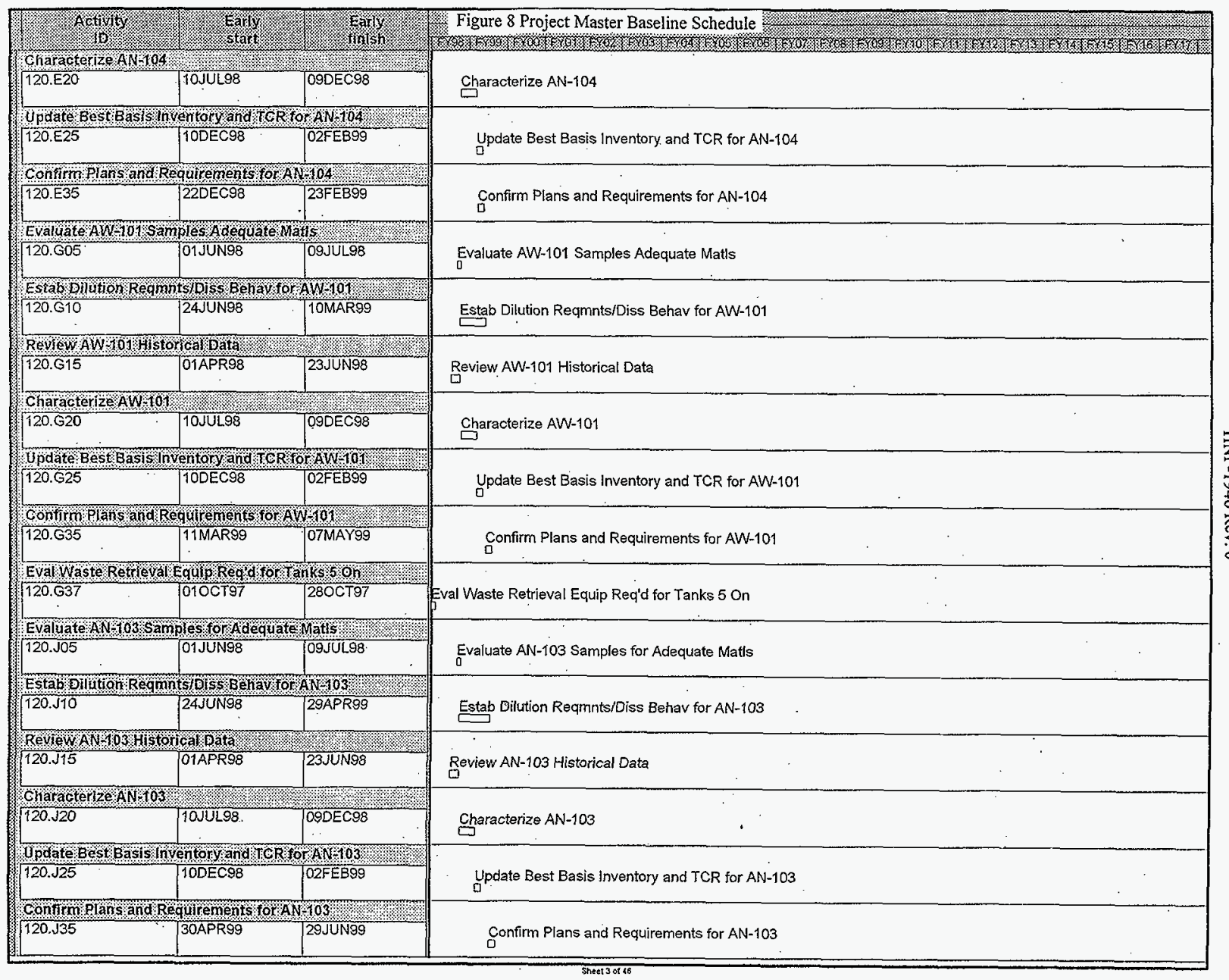




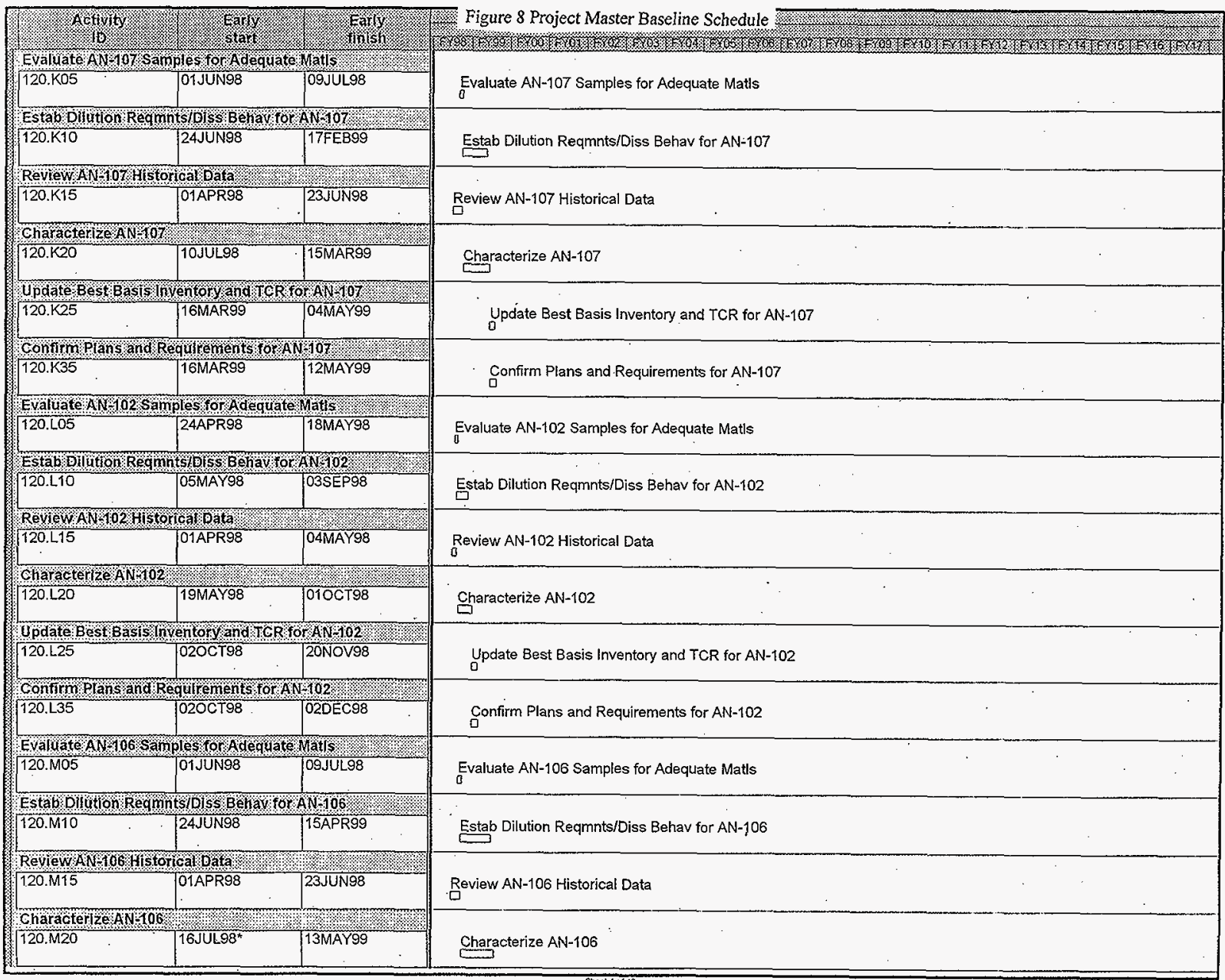




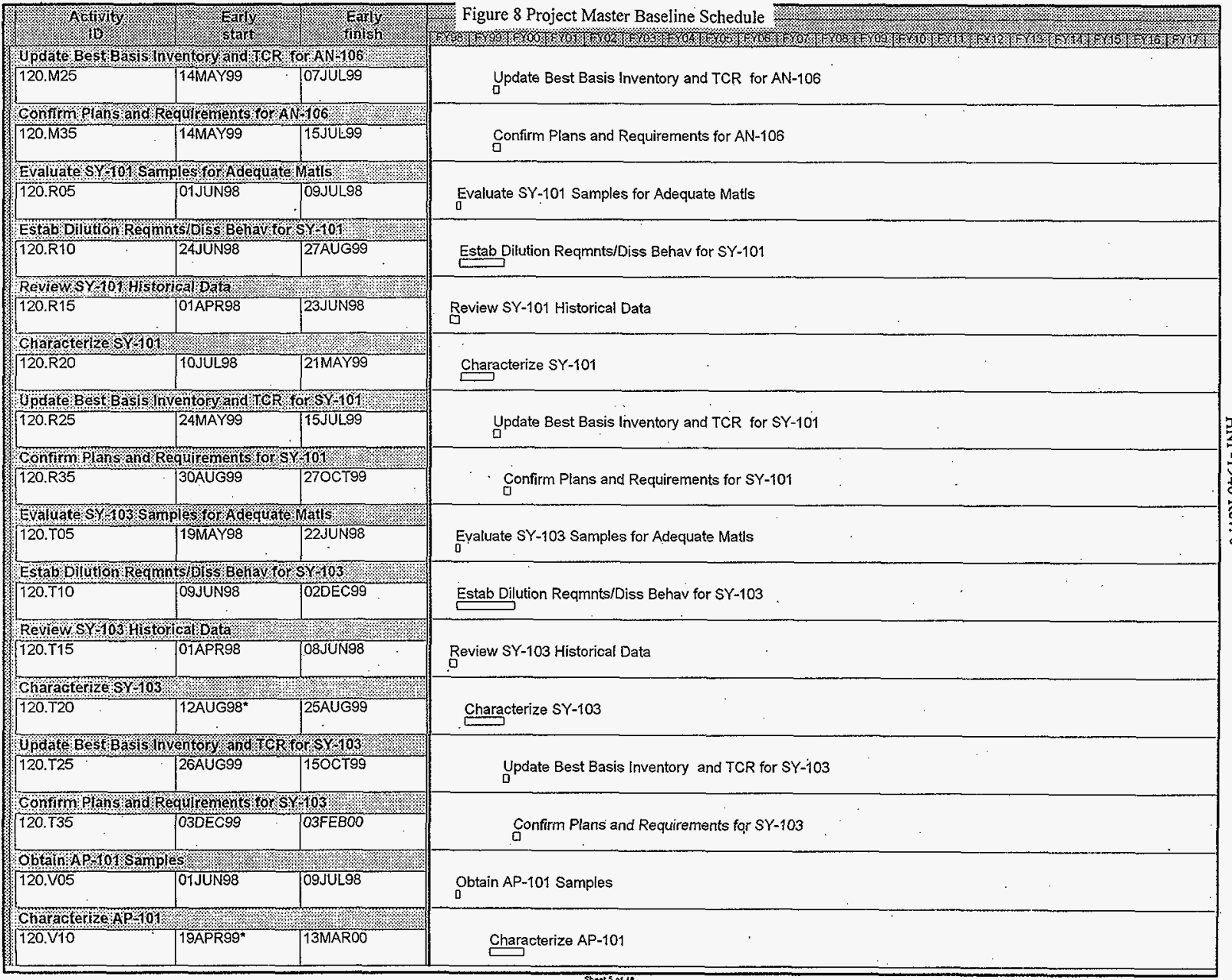




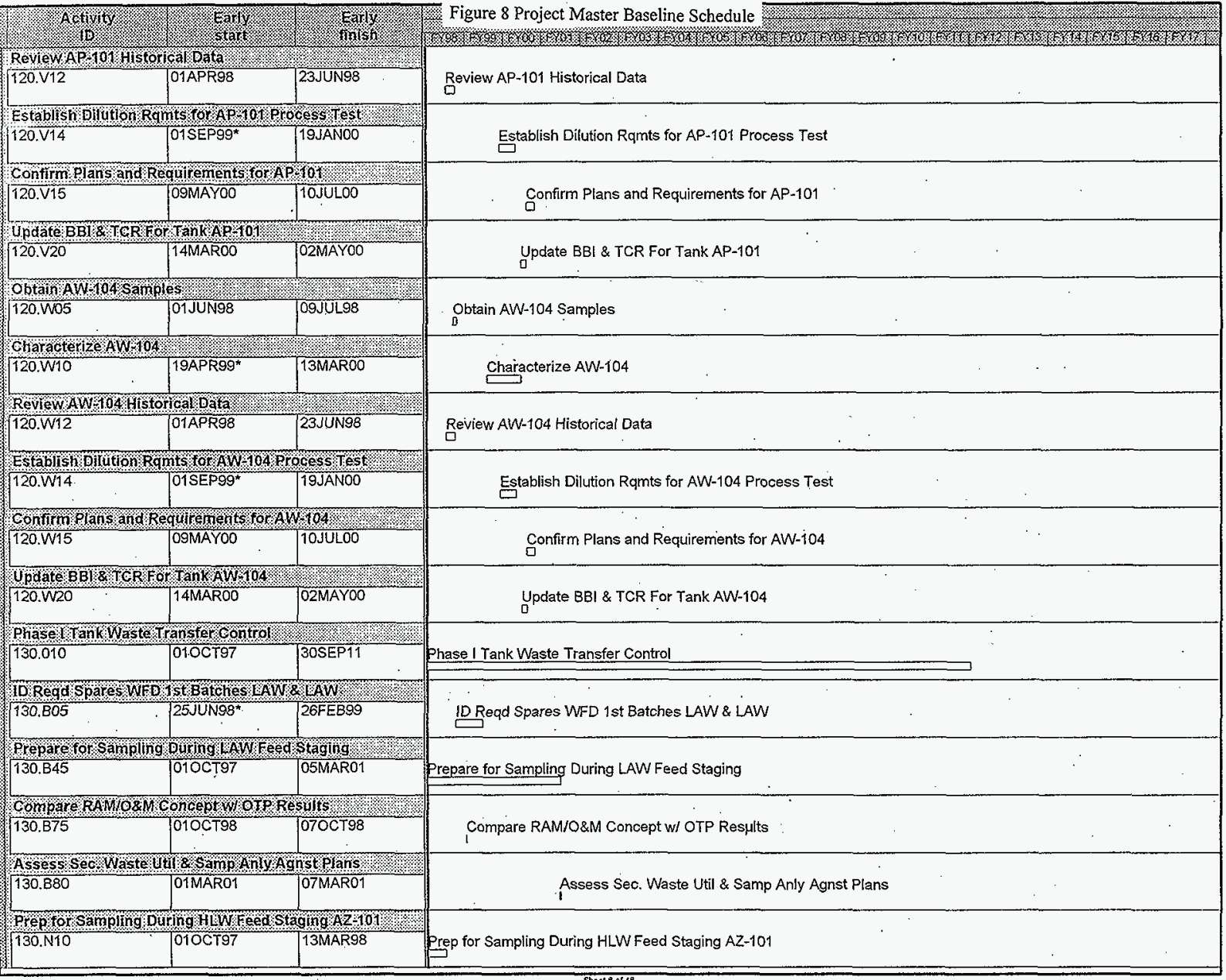




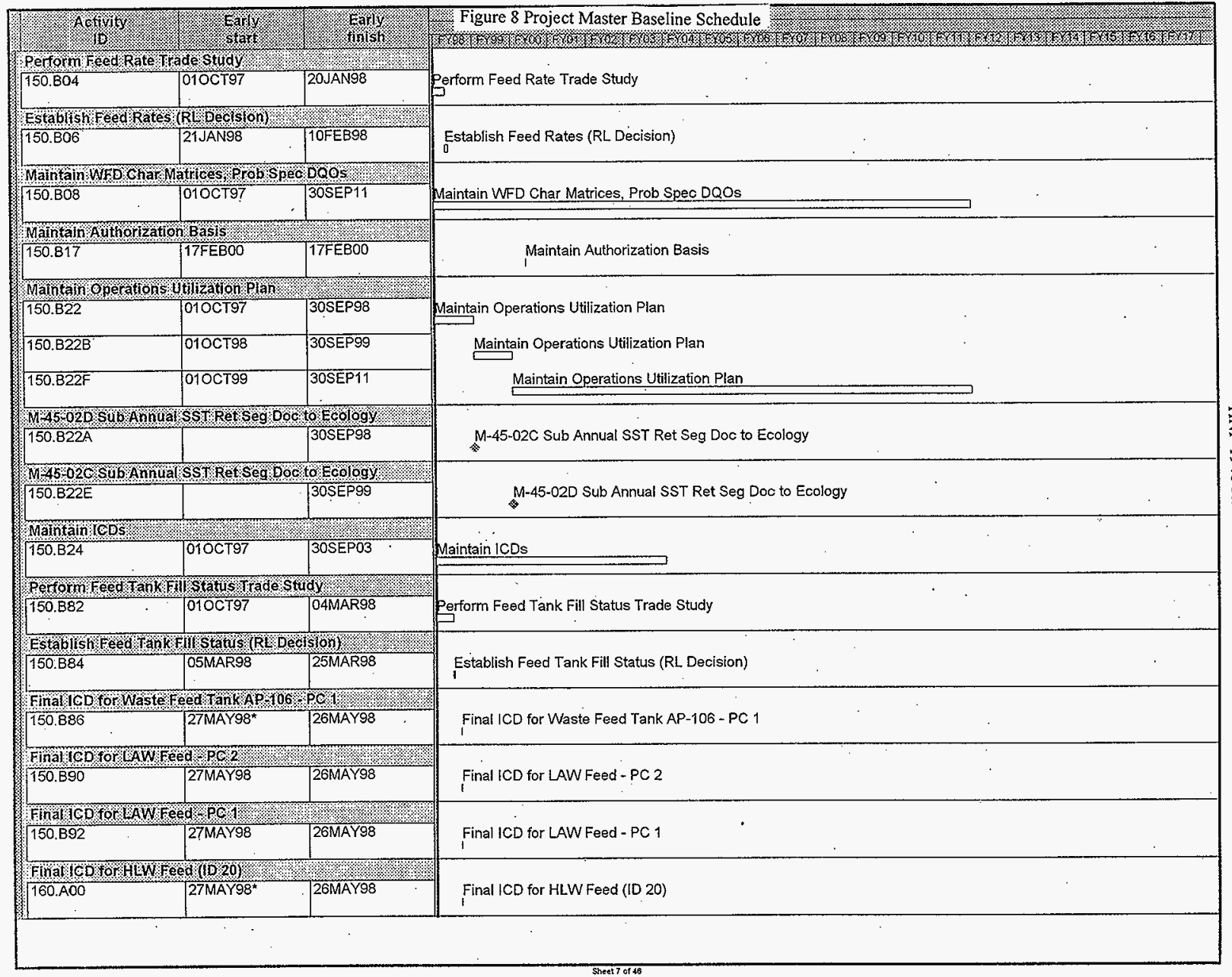




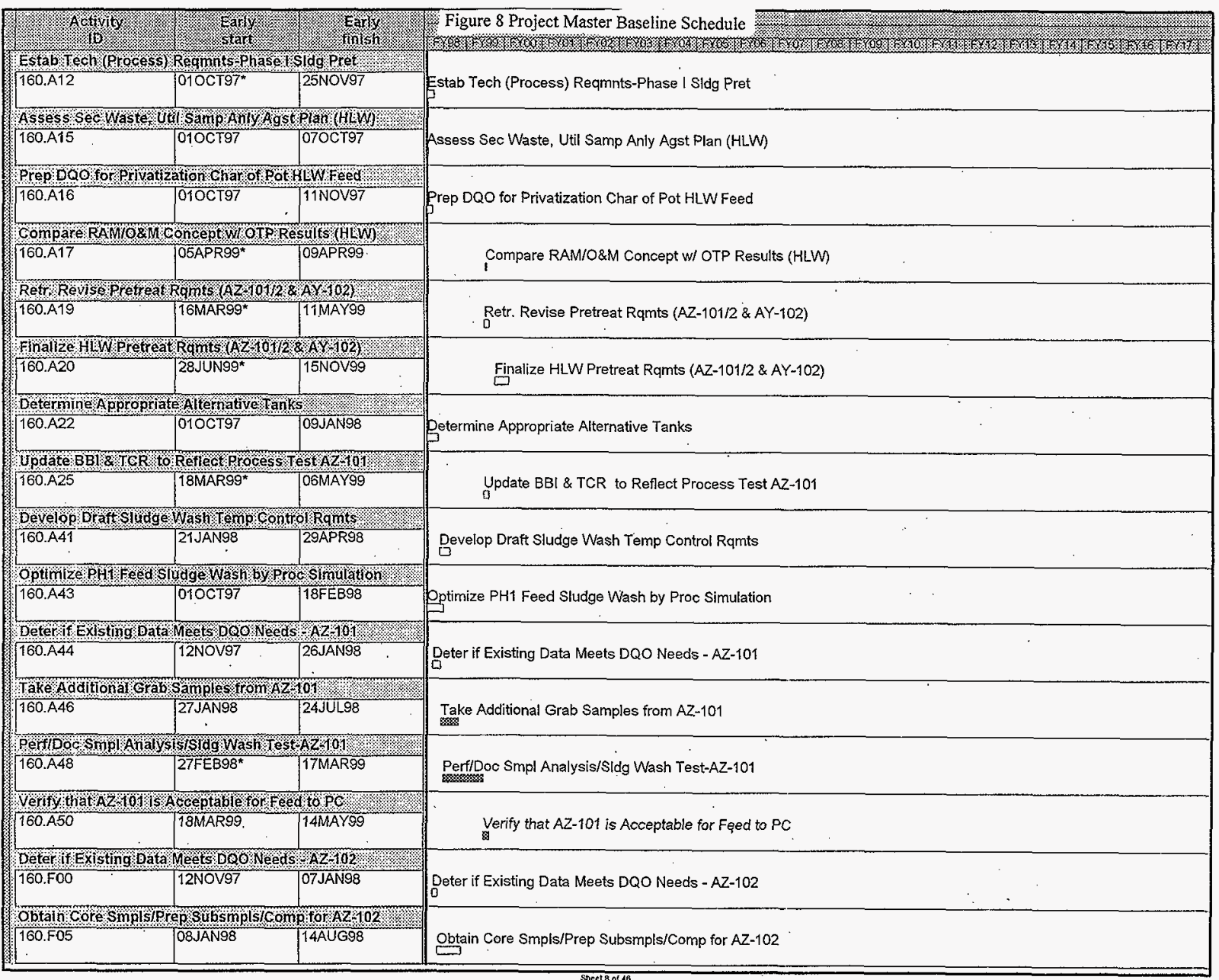




\begin{tabular}{|c|c|c|c|}
\hline 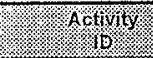 & 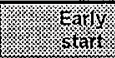 & 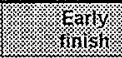 & Figure 8 Project Master Baseline Schedule \\
\hline \multicolumn{3}{|c|}{ Periooc Snpl Analys sistagWas 6 Testing $\mathrm{Az} 102$. } & \multirow{2}{*}{ Perf/Doc Smpl Analysis/SIdgWash Testing - AZ-102 } \\
\hline $160 . \mathrm{F} 10$ & $040 E C 97^{*}$ & 17 MAR99 & \\
\hline \multicolumn{3}{|c|}{ Update B Bl and LCR Io AZ 102 \% } & \multirow[b]{2}{*}{ Update BBI and TCR for $A Z-102$} \\
\hline $160 . F 12$ & 18MAR99* & $06 M A Y 99$ & \\
\hline \multicolumn{3}{|c|}{ 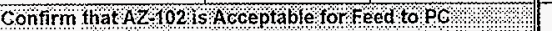 } & \multirow[b]{2}{*}{ Confirm that $A Z-102$ is Acceptable for Feed to PC } \\
\hline $160 . \mathrm{F15}$ & 18 MAR99 & 14MAY99 & \\
\hline \multicolumn{3}{|c|}{ PCConduct Waste Eorm $\alpha$ ol $/$ onnfirmation $A z-102$} & \multirow[b]{2}{*}{ PC Conduct Waste Form Qual Confirmation-AZ-102 } \\
\hline $160 . \mathrm{F} 80$ & $170 \mathrm{DCO} 2$ & 27AUGO3 & \\
\hline \multicolumn{3}{|c|}{ Deter If Existing DataMeets DQO Needs $/ \mathrm{Y}-102$} & \multirow[b]{2}{*}{ Deter if Existing Data Meets DQO Needs - AY-102 } \\
\hline $160 . \mathrm{HOO}$ & 27JAN98* & 19MAR98 & \\
\hline \multicolumn{3}{|c|}{ obto ooresmplsprep subsuplsicomp or AY 02 \% } & \multirow[b]{2}{*}{ Obtn Core Smpls/Prep Subsmpls/Comp for AY-102 } \\
\hline $160 . \mathrm{HOS}$ & 20MAR98 & TI2JANOO & \\
\hline \multicolumn{3}{|c|}{ PeffDocsmpl Analystsisidg Wash Testing $\mathrm{AY} / 02$ \% } & \multirow[b]{2}{*}{ Perf/Doo Smpl Analysis/Sldg Wash Testing-AY-102 } \\
\hline $160 . \mathrm{H} 20$ & 13JANOO & 27 SEPOO & \\
\hline \multicolumn{3}{|c|}{ UpdatesBland $T C R$ fo $\mathrm{A} Y 102$} & \multirow[b]{2}{*}{ Update BBI and TCR for AY-102 } \\
\hline $160 . \mathrm{H}_{22}$ & $020 \mathrm{CTOO}^{*}$ & 20NOVOO & \\
\hline \multicolumn{3}{|c|}{ 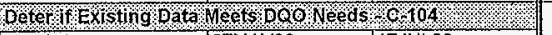 } & \multirow{2}{*}{ Deter if Existing Data Meets DQO Needs - C-104 } \\
\hline 160.J00 & 27 MAY98 & $17 \mathrm{JUL} 98$ & \\
\hline \multicolumn{3}{|c|}{ 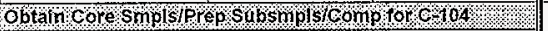 } & \multirow{2}{*}{ Obtain Core Smpls/Prep Subsmpls/Comp for C-104 } \\
\hline 160.305 & $20 J U L 98$ & 26FEB99 & \\
\hline \multicolumn{3}{|c|}{ 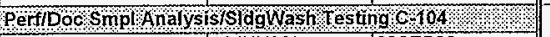 } & \multirow[b]{2}{*}{ Perf/Doc Smpl Analysis/SIdgWash Testing C-104 } \\
\hline $160 . \sqrt{10}$ & 18JUN98* & 29SEP99 & \\
\hline \multicolumn{3}{|c|}{ UpdateB BI And $T C B$ for $C 104 \%$} & \multirow[b]{2}{*}{ Update $\mathrm{BBI}$ and TCR for $\mathrm{C}-104$} \\
\hline $160 . \sqrt{12}$ & T010CT99 & 19NOV99 & \\
\hline \multicolumn{3}{|c|}{ Confirm that $c$ - $104 \mathrm{~s} A \mathrm{cceptab}$ e fot $\mathrm{e}$ ed to $\mathrm{PC}$} & \multirow[b]{2}{*}{ Confirm that C-104 is Acceptable for Feed to PC } \\
\hline $160 . \sqrt{15}$ & 010 CT99 & O1DEC99 & \\
\hline \multicolumn{3}{|c|}{ 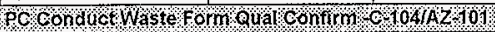 } & \multirow{2}{*}{ PC Conduct Waste Form Qual Confirm -C-104/AZ-101 } \\
\hline $160 . \sqrt{80}$ & 04APR06 & $12 \mathrm{DEC06}$ & \\
\hline \multicolumn{3}{|c|}{ Develoowastefed Delivery safer Basispo } & \multirow{2}{*}{ Pevelop Waste Feed Delivery Safety Basis DQO } \\
\hline 620.015 & $010 \mathrm{CT97}$ & 3TMAR98 & \\
\hline \multicolumn{3}{|c|}{ Mantain Waste Fead Definery system summary } & \\
\hline 620.020 & $0100 T 97$ & 30SEP11 & Maintain Waste Feed Delivery System Summary \\
\hline
\end{tabular}




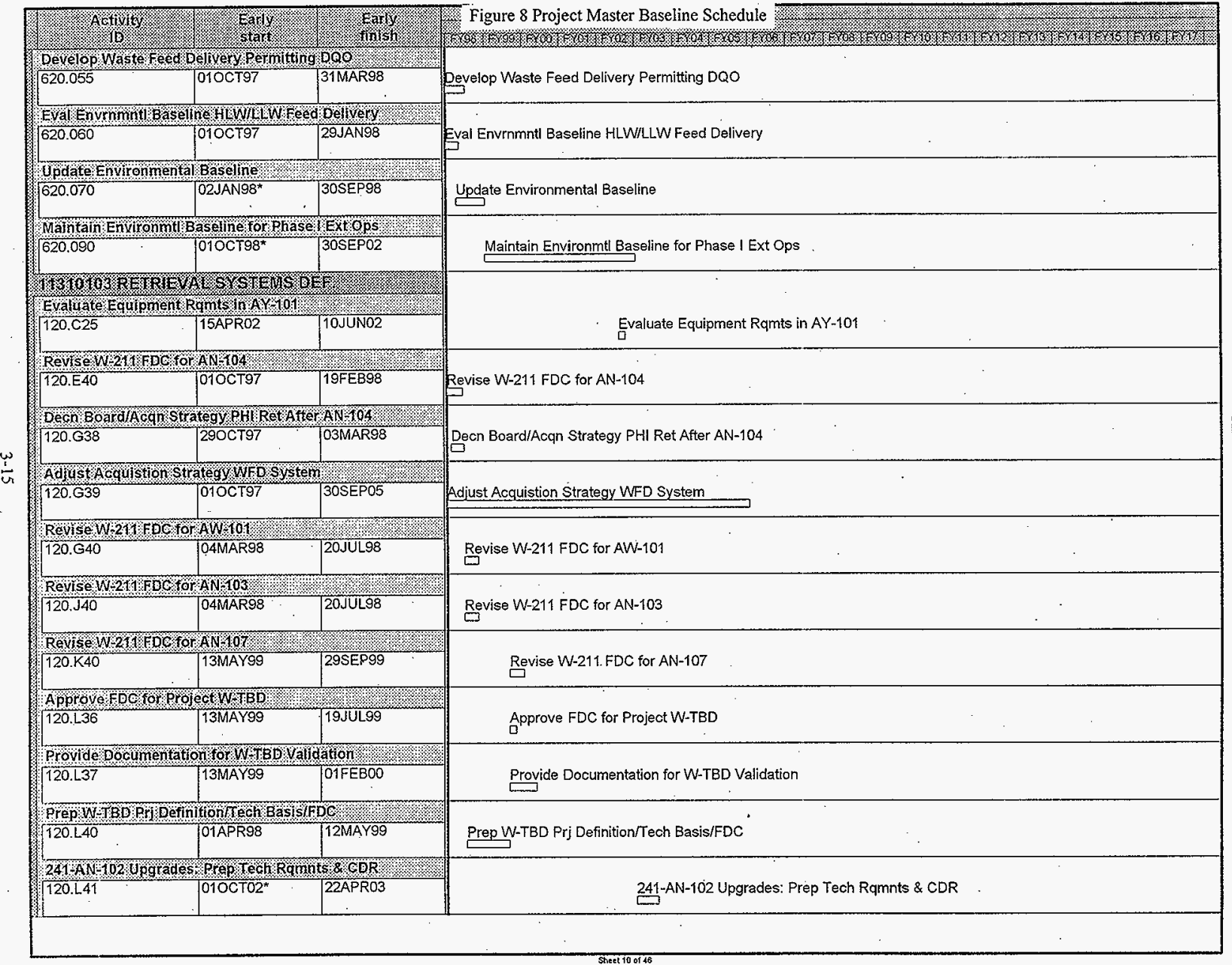




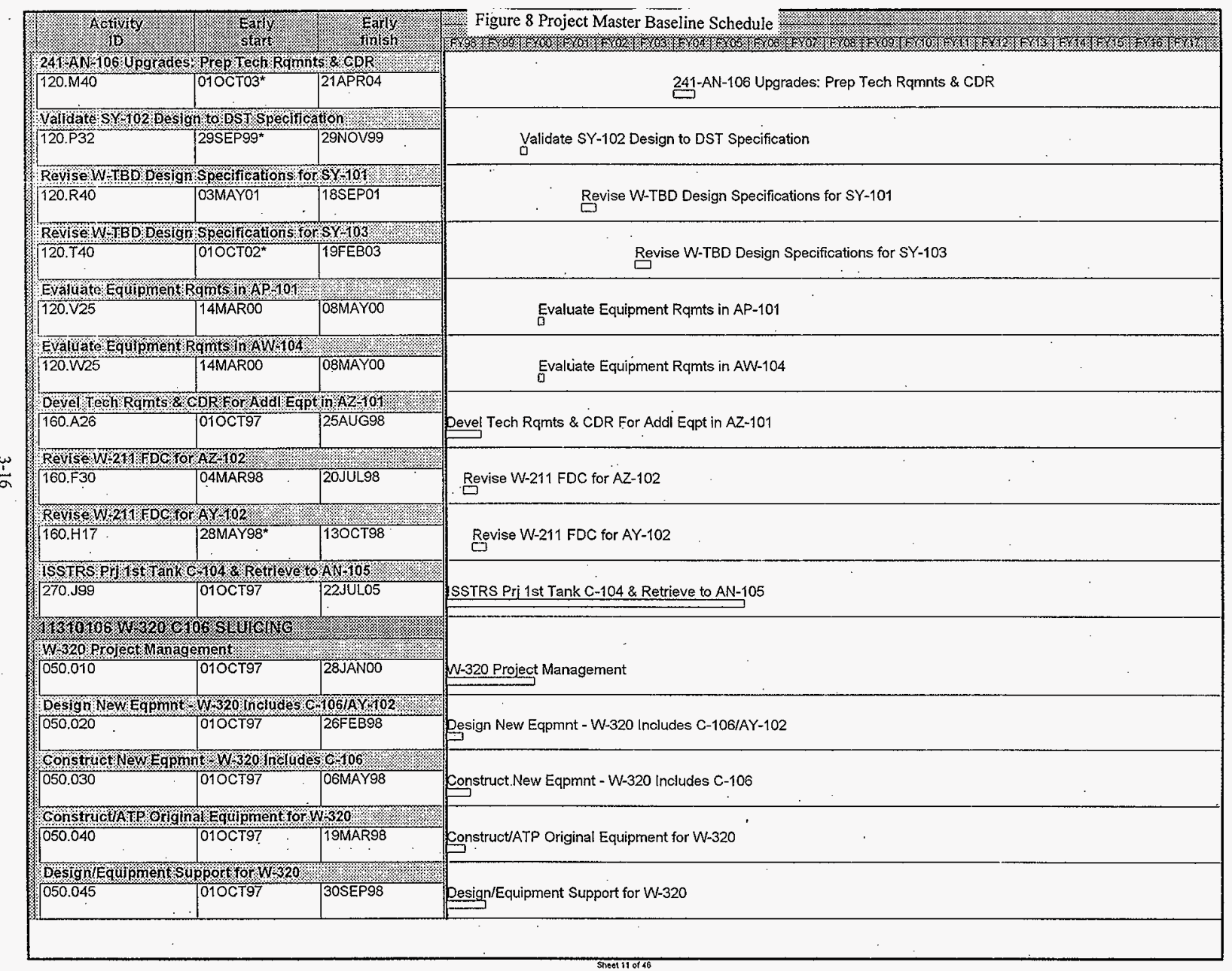




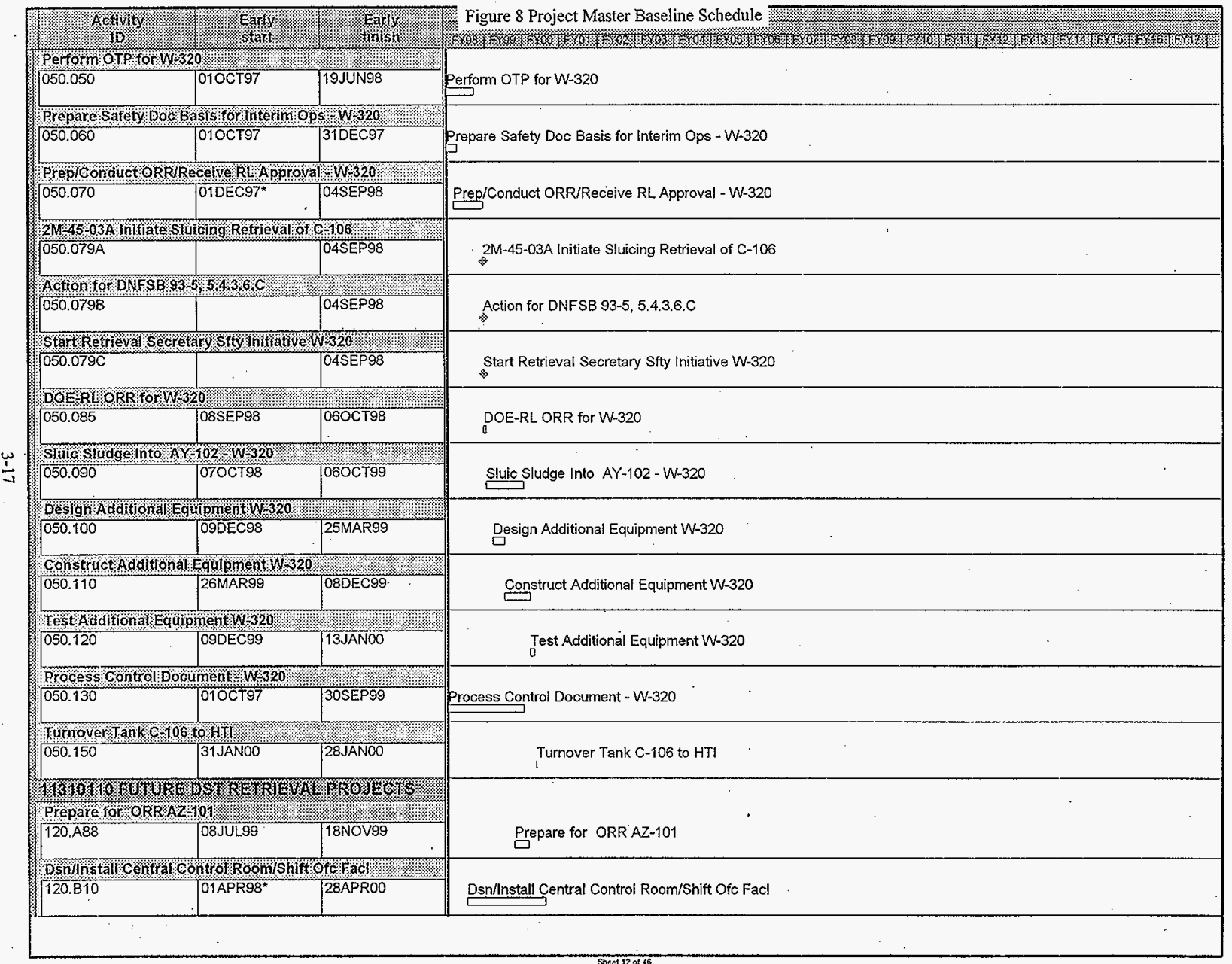




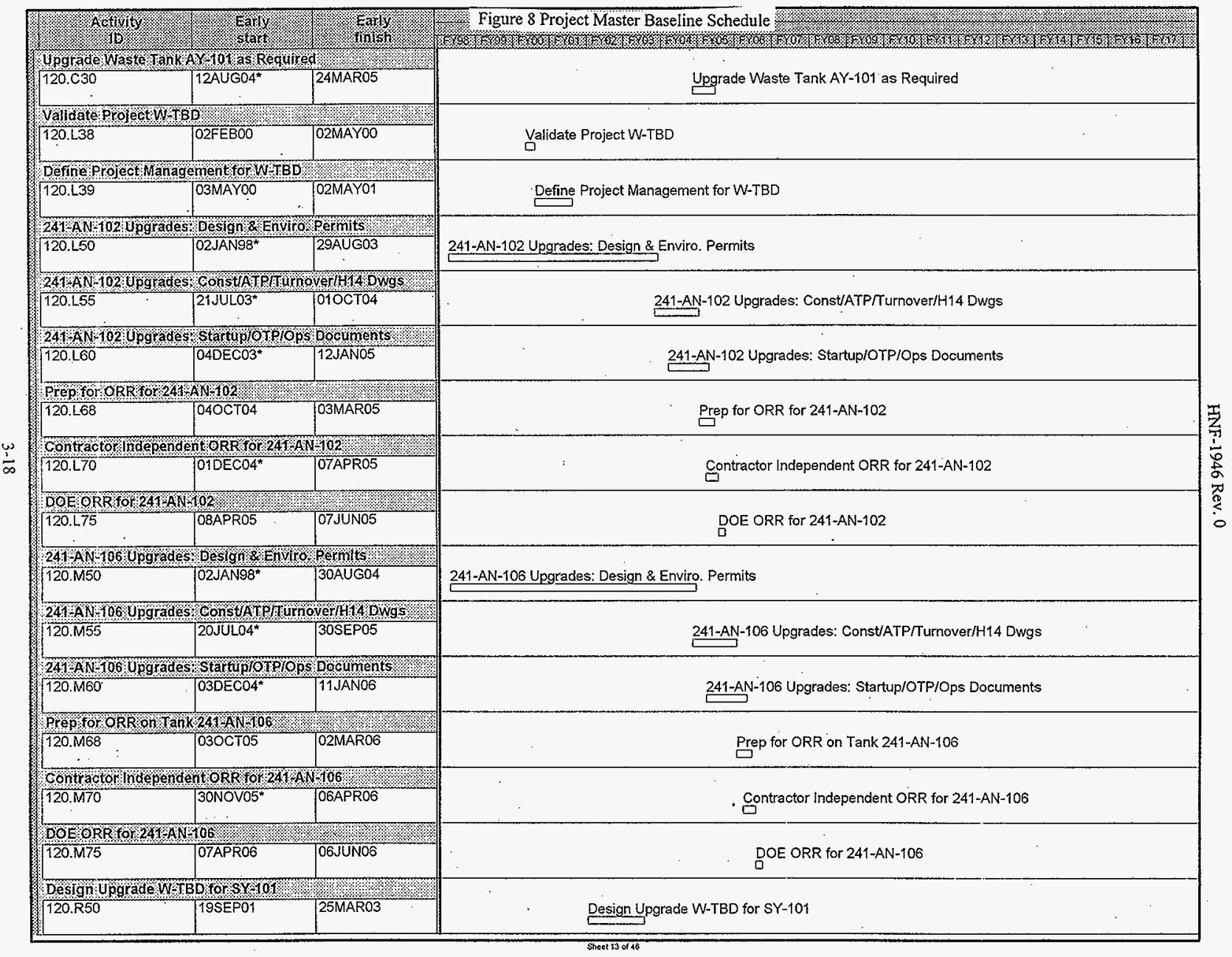




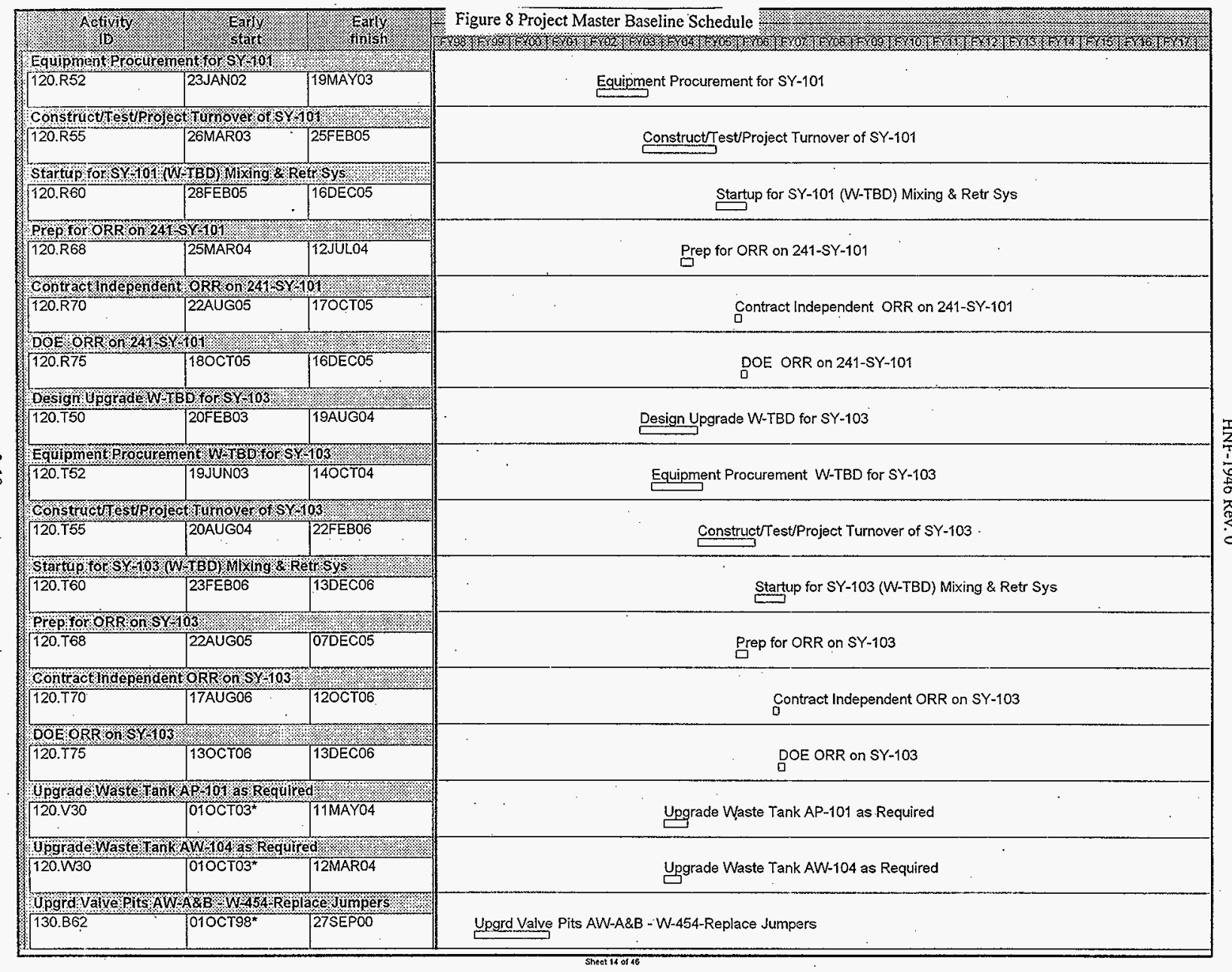




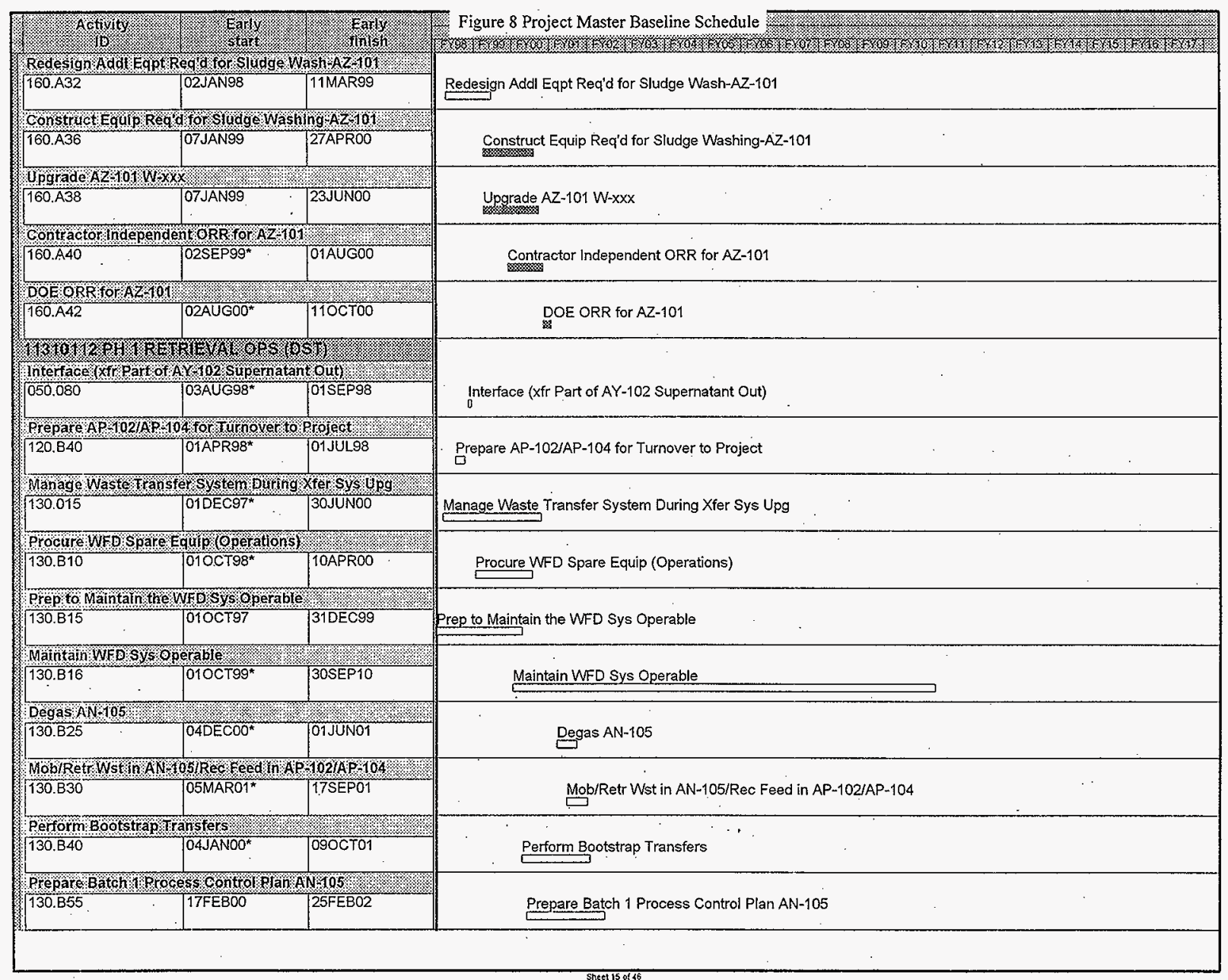




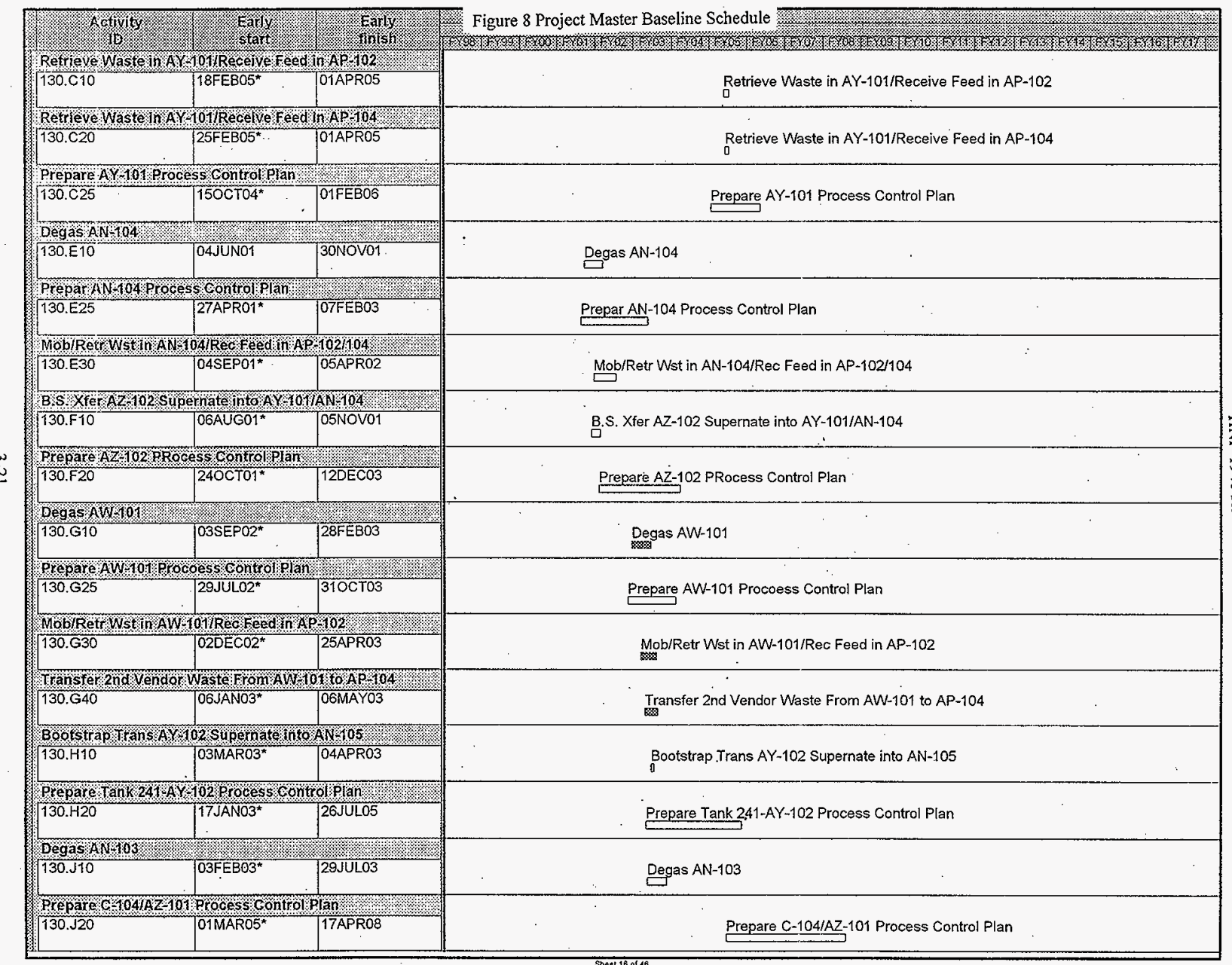




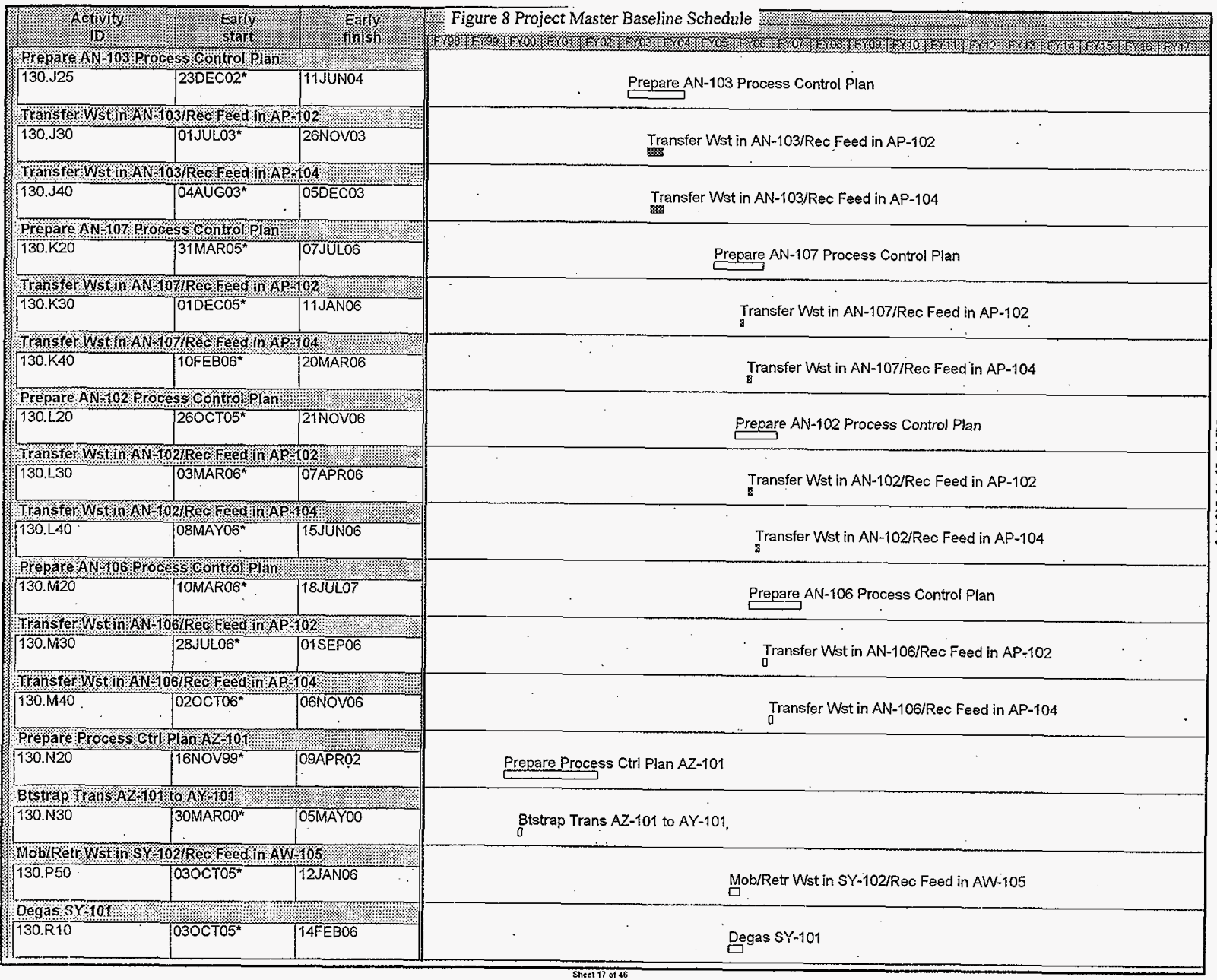




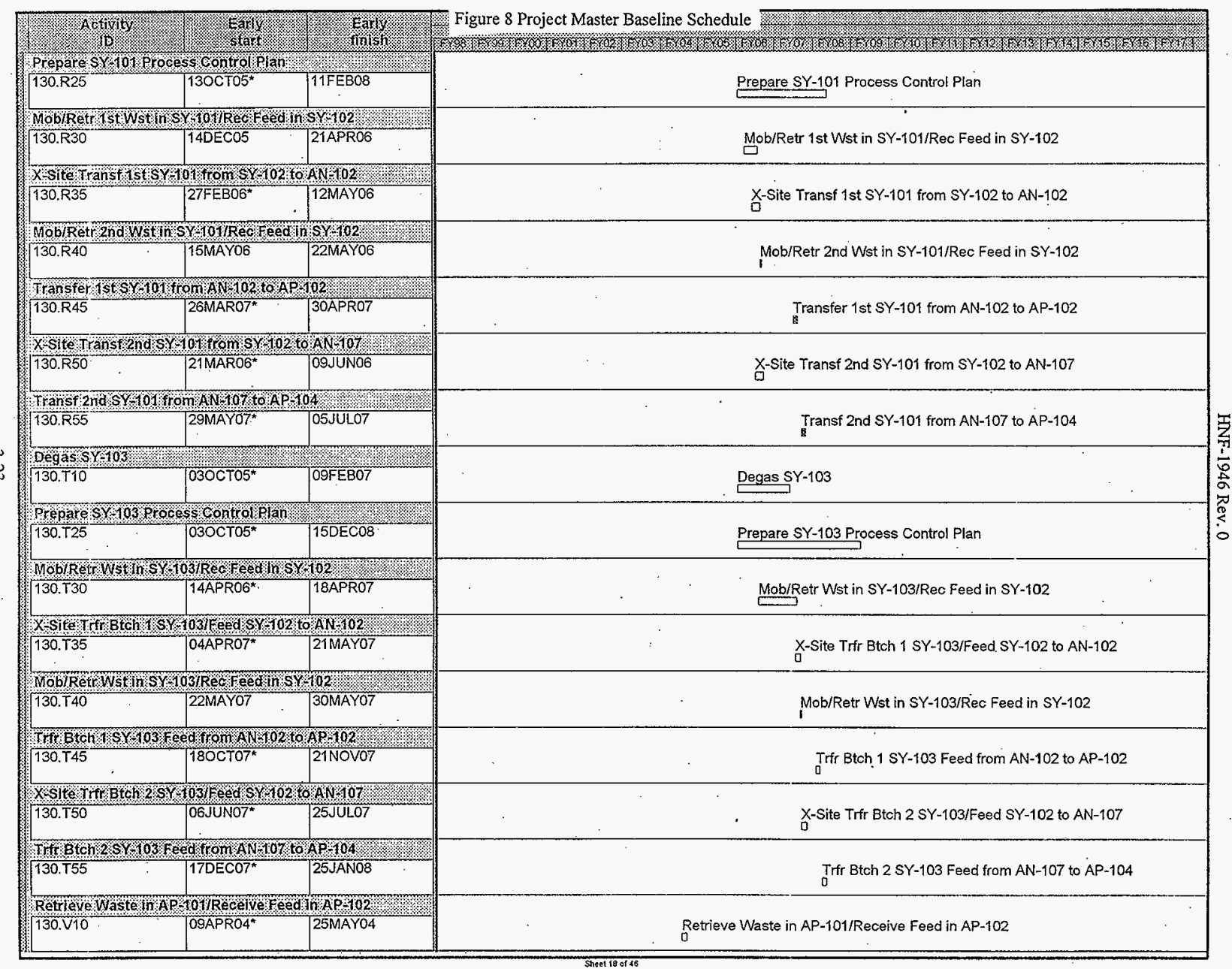




\begin{tabular}{|c|c|c|c|}
\hline 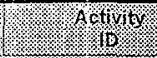 & W. & then & 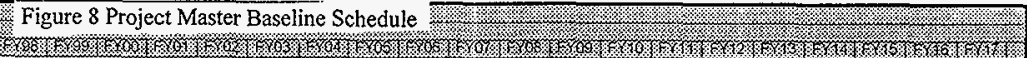 \\
\hline \multicolumn{3}{|c|}{ 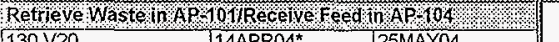 } & \multirow[b]{2}{*}{$\begin{array}{l}\text { Retrieve Waste in AP-101/Receive Feed in AP-104 } \\
0\end{array}$} \\
\hline $130 . \sqrt{ } 20$ & 14APRO4* & 25MAYO4 & \\
\hline \multicolumn{3}{|c|}{ PrepAP-101/AW 104 Batoh Process oontrolplan } & \multirow[b]{2}{*}{ Prep AP-101/AW-104 Batch Process Control Plan } \\
\hline $130 . \vee 25$ & $02 \mathrm{FEBO} 4^{\star}$ & 19APRO5 & \\
\hline \multicolumn{3}{|c|}{ Retrieve Waste in AW to4/Recelve Feed In AP 102} & \multirow[b]{2}{*}{$\begin{array}{l}\text { Retrieve Waste in AW-104/Receive Feed in AP-102 } \\
0\end{array}$} \\
\hline $130 . \mathrm{W} 10$ & 23APRO4* & O9JUNO4 & \\
\hline \multicolumn{3}{|c|}{ 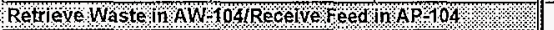 } & \multirow[b]{2}{*}{$\begin{array}{l}\text { Retrieve Waste in AW-104/Receive Feed in AP-104 } \\
\text { D }\end{array}$} \\
\hline $130 . \mathrm{W} 20$ & 28 APRO4* & D9JUN04 & \\
\hline \multicolumn{3}{|c|}{ 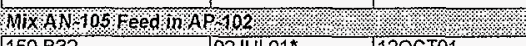 } & \multirow[b]{2}{*}{ Mix AN-105 Feed in AP-102 } \\
\hline 150.832 & O2JULO1* & $120 \mathrm{CT} 01$ & \\
\hline \multicolumn{3}{|c|}{ AdjustaN $105 \mathrm{Fed}$ In AP 102 , } & \multirow[b]{2}{*}{ Adjust AN-105 Feed in AP-102 } \\
\hline 150.842 & O4JUNO1^ & $090 \mathrm{CTO1}$ & \\
\hline \multirow{2}{*}{\multicolumn{3}{|c|}{ Deliver AN $105 \mathrm{Fed}$ to AP 106, }} & \multirow[b]{2}{*}{ Deliver AN-105 Feed to AP-106 } \\
\hline 150.844 & & 11JANO2 & \\
\hline \multicolumn{3}{|c|}{ WWRS EISRODHOLOP ont 2 2 } & \multirow[b]{2}{*}{ TWRS EIS ROD Hold Point 2} \\
\hline $150 . \mathrm{B} 46 \mathrm{H}$ & O3JUNO2* & $31 \mathrm{MAYO} 2$ & \\
\hline \multicolumn{3}{|c|}{ MiXAN-105 Feed foAP 104 } & \multirow[b]{2}{*}{$\mathrm{Mix}$ AN-105 Feed in AP-104 } \\
\hline $150 . \mathrm{B} 48$ & 02JULO1* & $2200 T 01$ & \\
\hline \multicolumn{3}{|c|}{ 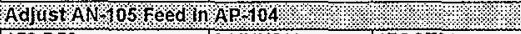 } & \multirow[b]{2}{*}{$\begin{array}{l}\text { Adjust AN-105 Feed in AP-104 } \\
\text { 口. }\end{array}$} \\
\hline $150 . \mathrm{B} 58$ & O4JUNO1* & $170 \mathrm{CTO1}$ & \\
\hline \multicolumn{3}{|c|}{ Deliver AN 105 FeedfoAP 108 , } & \multirow[b]{2}{*}{ Deliver AN-105 Feed to AP-108. } \\
\hline 150.860 & 15JANO2* & 23JANO2 & \\
\hline \multicolumn{3}{|c|}{ WRS EIS RODHOIARONT $\%$, } & \multirow[b]{2}{*}{ TWRS EIS ROD Hold Point 1} \\
\hline $150 . \mathrm{B} 63 \mathrm{H}$ & $16{\text { NOV } 98^{*}}^{\prime}$ & 13 NOV98 & \\
\hline \multicolumn{3}{|c|}{ 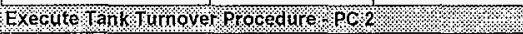 } & \multirow[b]{2}{*}{ Execute Tank Turnover Procedure - PC 2} \\
\hline 150.868 & 16 NOV98 & 16NOV98 & \\
\hline \multicolumn{3}{|c|}{ RL Declates A P ro \& Ready Contractó ? } & \multirow[b]{2}{*}{ RL Declares AP-108 Ready, - Contractor 2} \\
\hline 150.870 & O4JANO1* & $02 \mathrm{JULO1}$ & \\
\hline \multicolumn{3}{|c|}{$\begin{array}{l}\text { Execute Tank Turnover Procedure POC } \\
150878\end{array}$} & \multirow[b]{2}{*}{ Execute Tank Turnover Procedure - PC 1} \\
\hline 150.878 & 16 NOV98 & 16 NOV98 & \\
\hline \multicolumn{3}{|c|}{ RIDeclares AP-16 6 Ready Coritractor 11} & \\
\hline 150.880 & O4.JANO ${ }^{*}$ & O2JUL01 & RL Délares AP-106 Ready - Contractor 1 \\
\hline
\end{tabular}




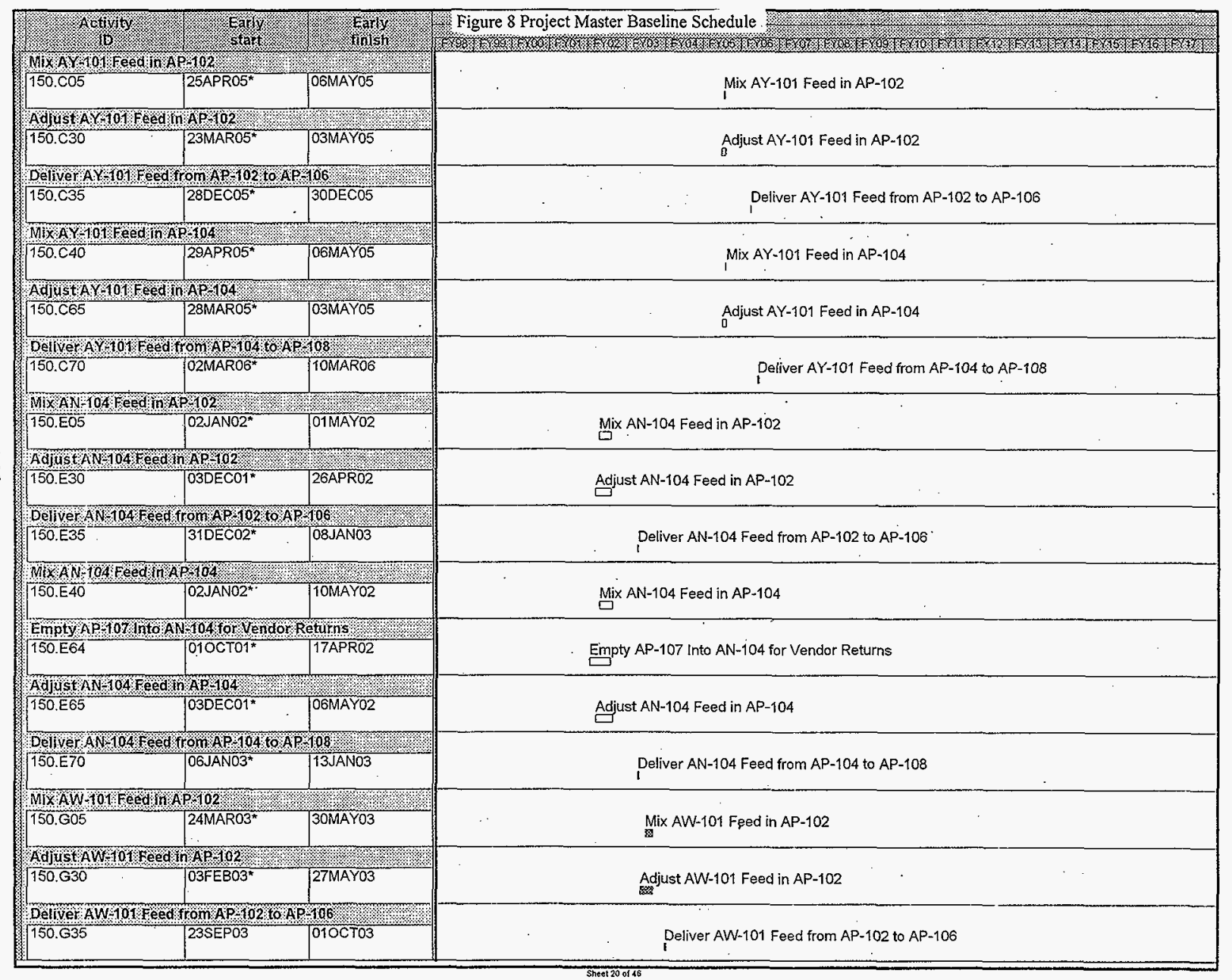




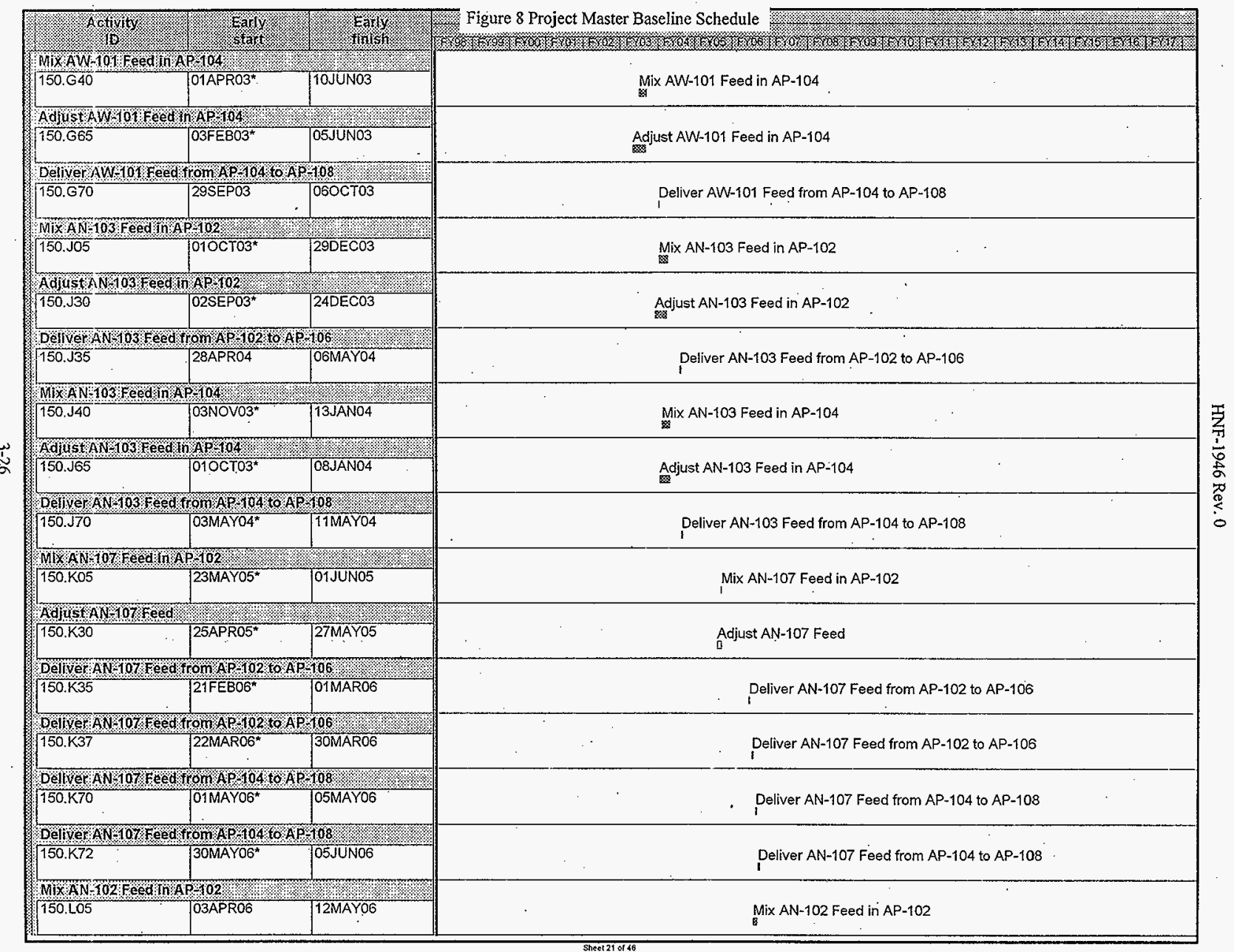




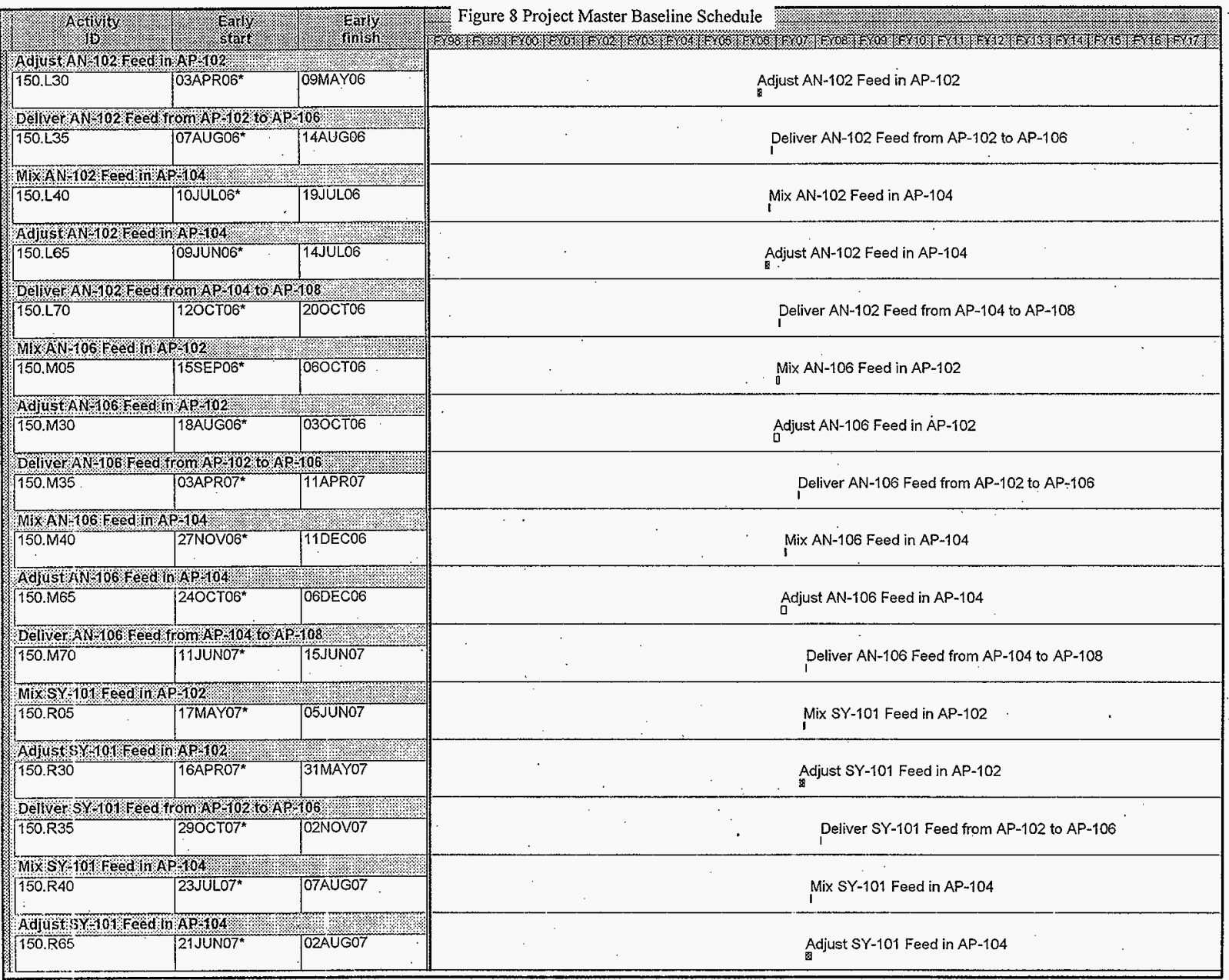




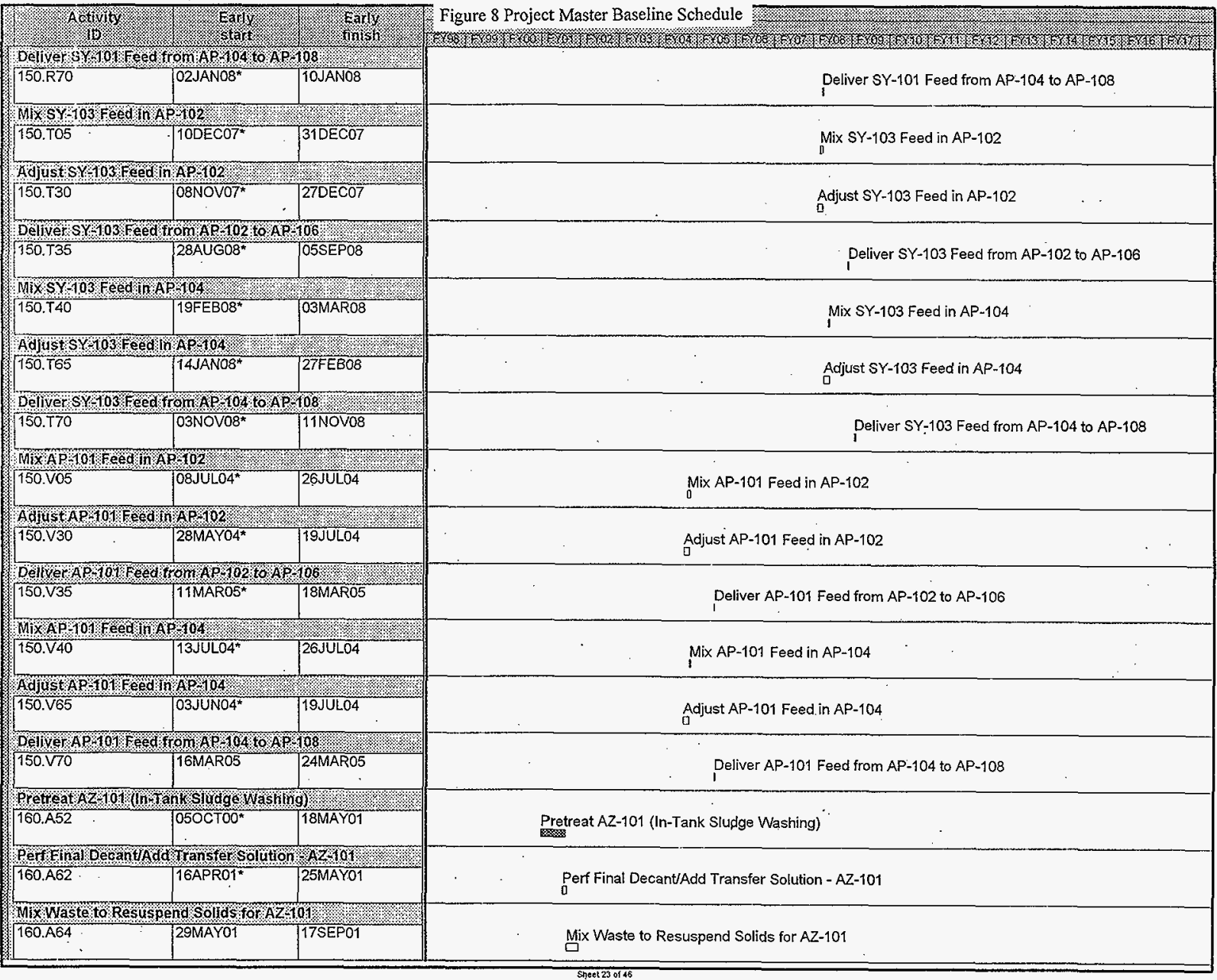




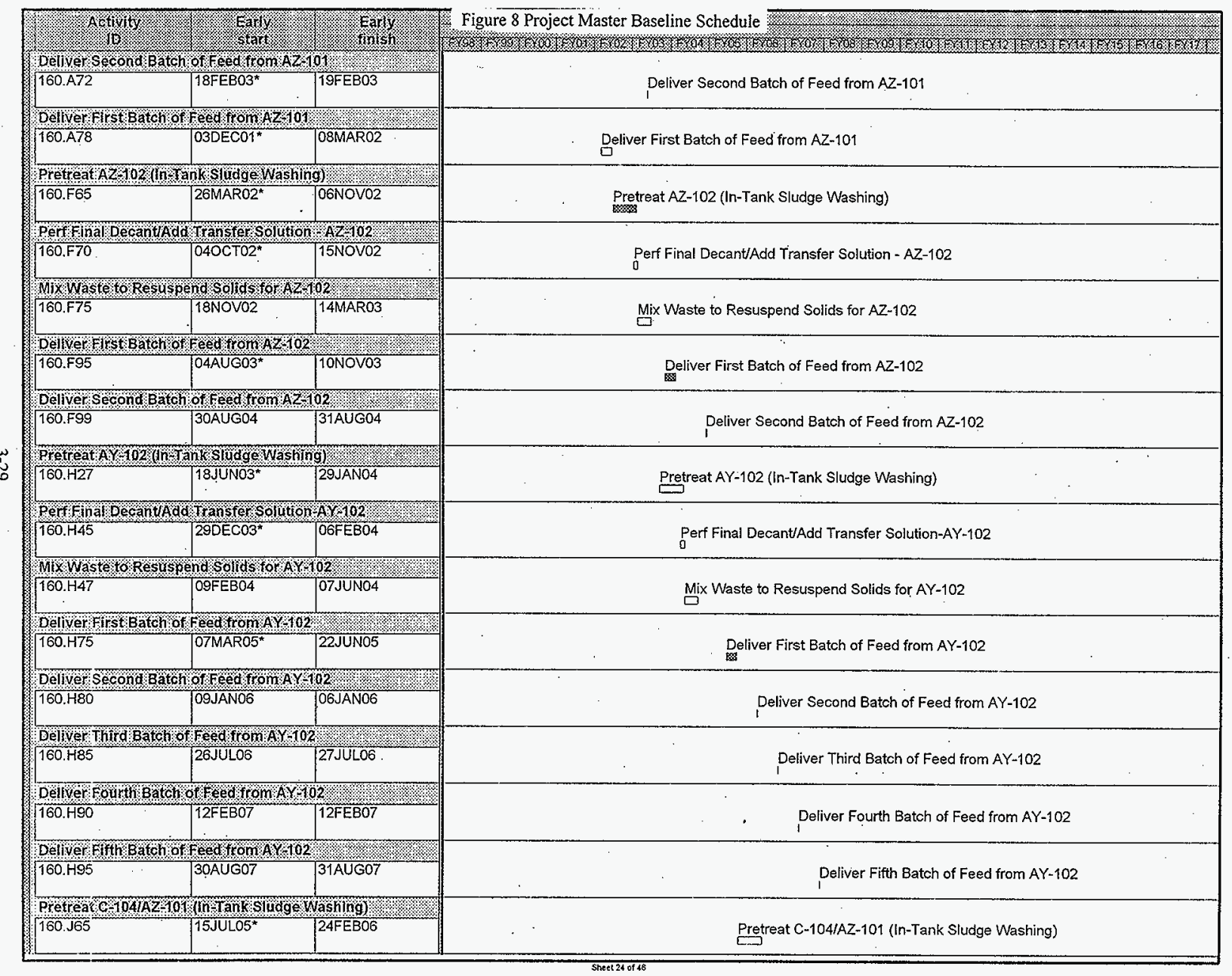




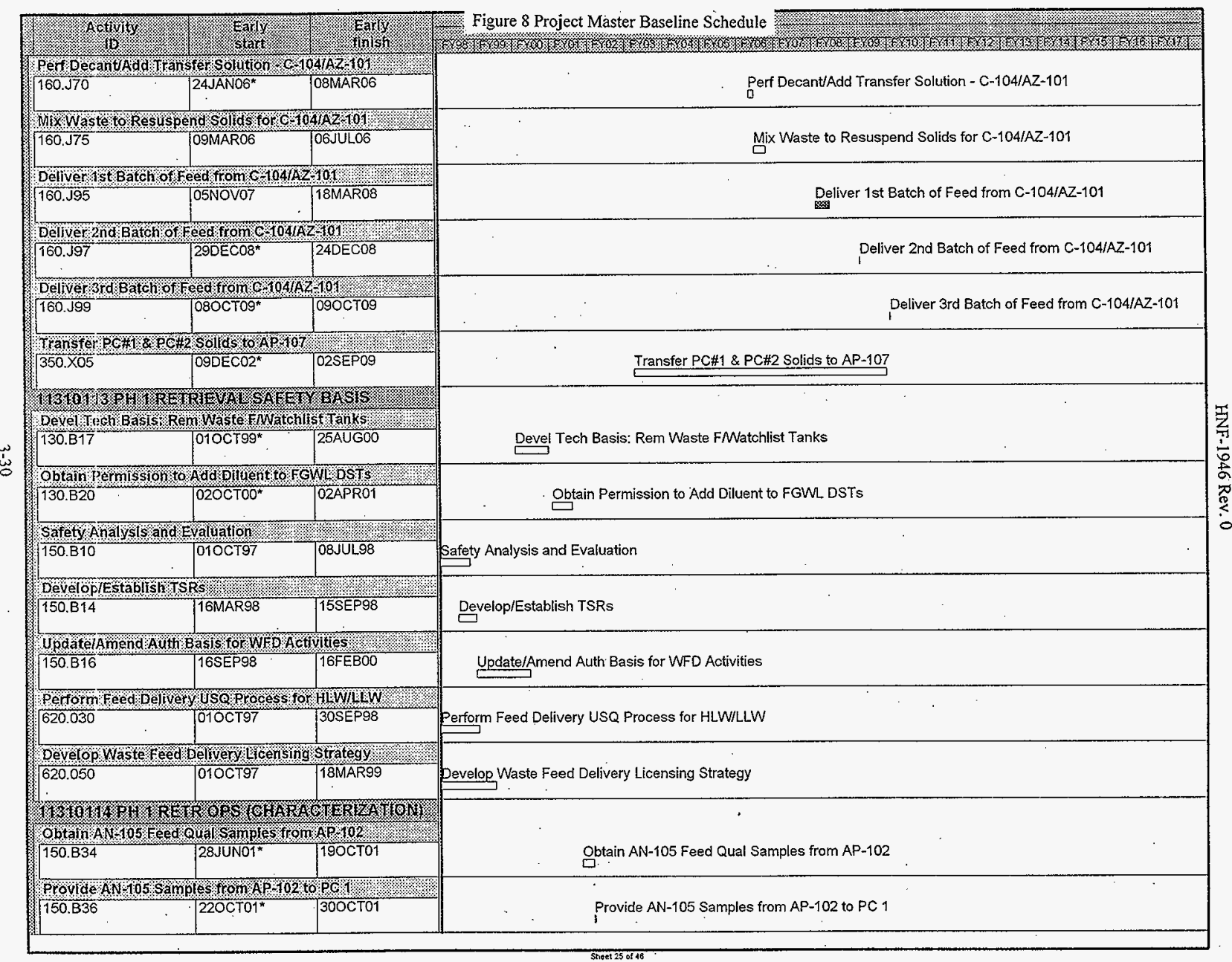




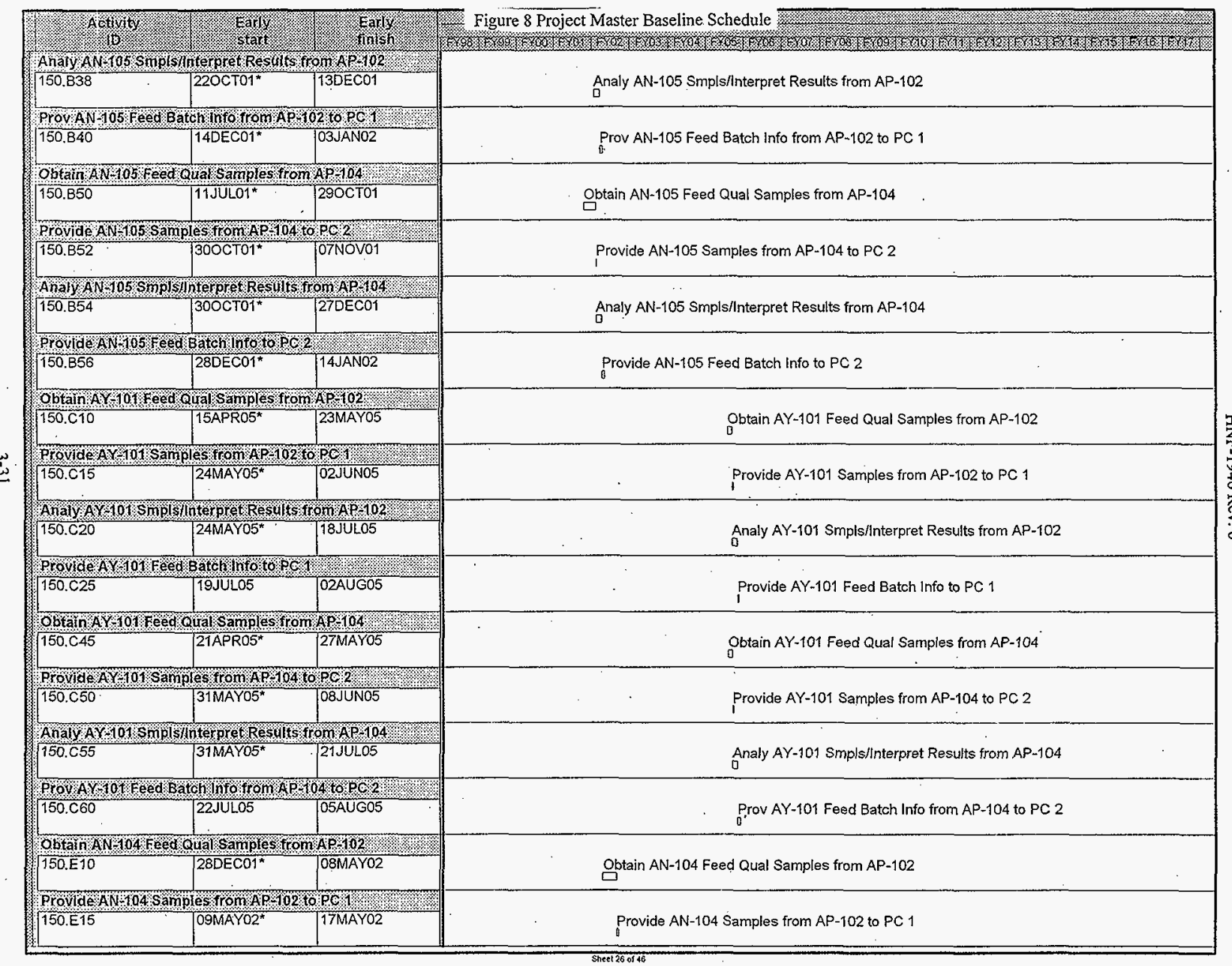




\begin{tabular}{|c|c|c|c|}
\hline (n) & 3. & 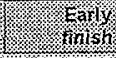 & Figure 8 Project Master Baseline Schedule \\
\hline Analy AN 104 & interpre Res & OM $A P 102$ & \multirow[b]{2}{*}{ Analy AN-104 Smpls/interpret Results from AP-102 } \\
\hline 150.E20 & $09 M A Y O 2^{*}$ & 01JUL02 & \\
\hline \multicolumn{3}{|c|}{ 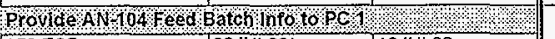 } & \multirow[b]{2}{*}{ Provide AN-104 Feed Batch Info to PC 1} \\
\hline $150 . \mathrm{E} 25$ & $02 \mathrm{JULO} 2^{*}$ & $18 J U L 02$ & \\
\hline \multicolumn{3}{|c|}{ obtain $A N$. 04 feed Qual S amples from AP $104 \%$. } & \multirow[b]{2}{*}{ Obtain AN-104 Feed Qual Samples from AP-104 } \\
\hline $150 . \mathrm{E} 45$ & 24JANO2* & 17MAYO2 & \\
\hline \multicolumn{3}{|c|}{ Provide AN 104 samples fom $A P$ to4 to $\mathrm{PC}_{2}$, } & \multirow[b]{2}{*}{ Provide AN-104 Samples from AP-104 to PC 2} \\
\hline $150 . \mathrm{E} 50$ & $20 M A Y O 2^{*}$ & 29MAYO2 & \\
\hline \multicolumn{3}{|c|}{ 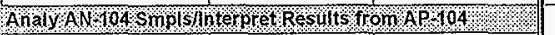 } & \multirow[b]{2}{*}{ Analy AN-104 Smpls/nterpret Results from AP-104 } \\
\hline $150 . E 55$ & 2OMAYO2 & 11JULO2 & \\
\hline \multicolumn{3}{|c|}{ 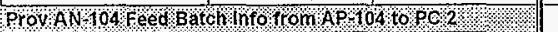 } & \multirow[b]{2}{*}{ Prov AN-104 Feed Batch Info from AP-104 to PC 2} \\
\hline $150 . \mathrm{E} 60$ & $12 \mathrm{JULO}{ }^{*}$ & 26JUL02 & \\
\hline \multicolumn{3}{|c|}{ 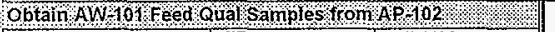 } & \multirow[b]{2}{*}{ Obtain AW-101 Feed Qual Samples from AP-102 } \\
\hline 150.610 & $27 \mathrm{FEBO3}{ }^{*}$ & TOSJUNO3 & \\
\hline \multicolumn{3}{|c|}{ Provide AWHol Samples from AP 102 to PCH 1} & \multirow[b]{2}{*}{ Provide AW-101 Samples from AP-102 to PC 1} \\
\hline 150.615 & 09JUN03* & 17 JUN03 & \\
\hline \multicolumn{3}{|c|}{ AndyAW 101 Smplsinter ret Resuls from AR 102 . } & \multirow[b]{2}{*}{ Analy AW-101 Smpls/nterpret Results from AP-102 } \\
\hline $150.620^{\circ}$ & 09JUNO $3^{*}$ & 31JULO3 & \\
\hline \multicolumn{3}{|c|}{ 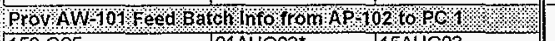 } & \multirow[b]{2}{*}{ Prov AW-101 Feed Batch Info from AP-102 to PC 1} \\
\hline 150.625 & $01 \mathrm{AUGO3^{* }}$ & 15AUG03 & \\
\hline \multicolumn{3}{|c|}{ obtain AW $101 \mathrm{Feed}$ oubl Samplessfom AP 104 . } & \multirow[b]{2}{*}{$\begin{array}{l}\text { Obtain AW-101 Feed Qual Samples from AP-104 } \\
\text { a }\end{array}$} \\
\hline 150.645 & OBMARO3* $^{*}$ & TO1JULO3 & \\
\hline \multicolumn{3}{|c|}{ ProvideAW-tols samples from AP 104 to PC 2, } & \multirow[b]{2}{*}{ Provide AW-101 Samples from AP-104 to PC 2} \\
\hline 150.650 & $02 J U L O 3^{*}$ & $14 J U L 03$ & \\
\hline \multicolumn{3}{|c|}{ Analy AW TO 1 Snplsiln teroresResults from AP-104, } & \multirow[b]{2}{*}{ - Analy AW-101 Smpls/nterpret Results from AP-104 } \\
\hline 150.655 & $02 \mathrm{JUL} 03^{*}$ & $22 \mathrm{AUG} 03$ & \\
\hline \multicolumn{3}{|c|}{ 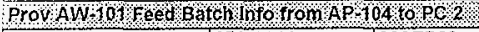 } & \multirow[b]{2}{*}{ Prov AW-10,1 Feed Batch Info from AP-104 to PC 2} \\
\hline $150 . G 60$ & 25AUG03 & OOSEP03 & \\
\hline \multicolumn{3}{|c|}{ 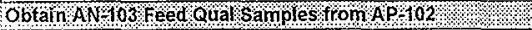 } & \multirow[b]{2}{*}{ Obtain AN-103 Feed Qual Samples from AP-102 } \\
\hline $150 . \sqrt{10}$ & 29SEP03* & O6JAN04 & \\
\hline \multicolumn{3}{|c|}{$\begin{array}{l}\text { Provide A N FO3 Samples fom AP } 102 \text { to PC } \\
\frac{150 \sqrt{15}}{15 J A N O 4}\end{array}$} & \\
\hline $150 . J 15$ & 07 JANO ${ }^{*}$ & 15JANO4 & Provide AN-103 Samples from AP-102 to PC 1 \\
\hline
\end{tabular}




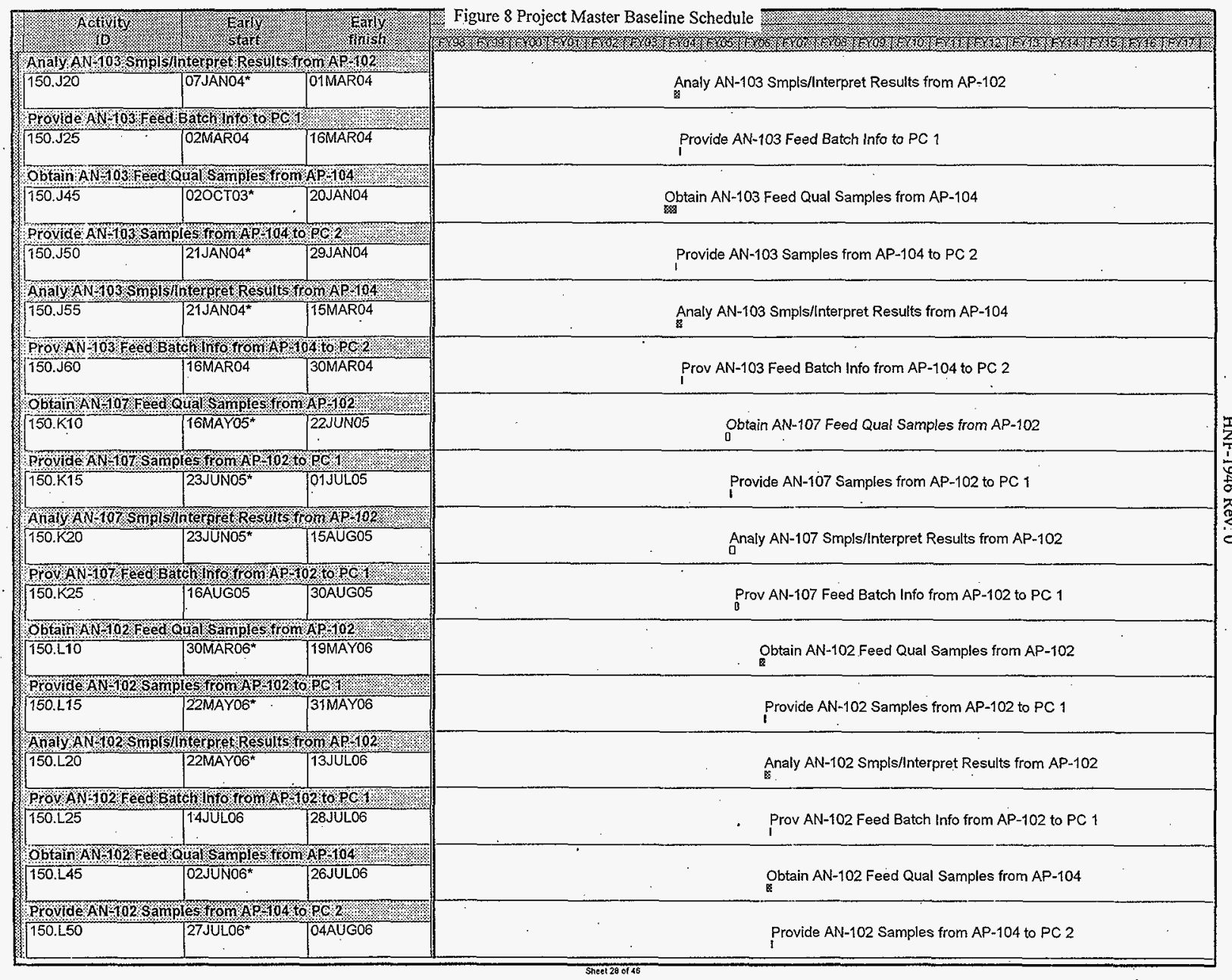




\begin{tabular}{|c|c|c|c|c|}
\hline \multicolumn{3}{|c|}{ 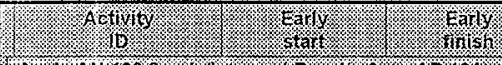 } & \multicolumn{2}{|c|}{ Figure 8 Project Master Baseline Schedule } \\
\hline \multicolumn{3}{|c|}{ Analy AN102 SMp IsInte pret Results tom AP 104} & \multirow{2}{*}{\multicolumn{2}{|c|}{ Analy AN-102 Smpis/interpret Results from AP-104 }} \\
\hline $150 . \mathrm{LS5}$ & 27 JULO6* & $19 S E P O 6$ & & \\
\hline \multicolumn{3}{|c|}{ 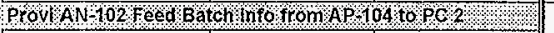 } & \multirow{2}{*}{\multicolumn{2}{|c|}{ Provi AN-102 Feed Batch Info from AP-104 to PC 2}} \\
\hline 150.560 & $20 S E P 06$ & 040CT06 & & \\
\hline \multicolumn{3}{|c|}{ 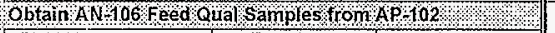 } & \multirow{2}{*}{\multicolumn{2}{|c|}{$\begin{array}{l}\text { Obtain AN-106 Feed Qual Samples from AP-102 } \\
0\end{array}$}} \\
\hline $150 . \mathrm{M} 10$ & 11SEP06* & $170 \mathrm{TTO}$ & & \\
\hline \multicolumn{3}{|c|}{ rovideAN-106samples tromap 102 tod $\alpha$ o } & \multirow{2}{*}{\multicolumn{2}{|c|}{ Provide AN-106 Samples from AP-102 to PC 1}} \\
\hline $150 . M 15$ & $180 C T 06^{*}$ & $260 \mathrm{CTO6}$ & & \\
\hline \multicolumn{3}{|c|}{ 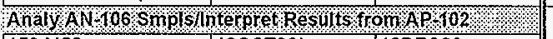 } & \multirow{2}{*}{\multicolumn{2}{|c|}{ Analy AN-106 Smpls/Interpret Results from AP-102 }} \\
\hline $150 . \mathrm{M} 20$ & $180 C \mathrm{TOG}^{*}$ & $12 \mathrm{DECO6}$ & & \\
\hline \multicolumn{3}{|c|}{ ProvAN 106 fed Batch nfo from Ap 102 to Po $1 /$} & \multirow{2}{*}{\multicolumn{2}{|c|}{ Prov AN-106 Feed Batch Info from AP-102 to PC 1}} \\
\hline $150 . \mathrm{M} 25$ & 13DECO6 & $29 D E C 06$ & & \\
\hline \multicolumn{3}{|c|}{ Obtain AN 106 Fed Qualsamples fom AP 104} & \multirow{2}{*}{\multicolumn{2}{|c|}{ Obtain AN-106 Feed Qual Samples from AP-104 }} \\
\hline $150 . \mathrm{M} 45$ & $17 N_{0 V 06}$ & 29DECO6 & & \\
\hline \multicolumn{3}{|c|}{ 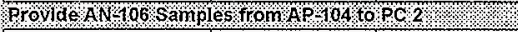 } & \multirow{2}{*}{\multicolumn{2}{|c|}{ Provide AN-106 Samples from AP-104 to PC 2}} \\
\hline $150 . M S 0$ & O2JANO $7^{\star}$ & $10 \mathrm{JANO}$ & & \\
\hline \multicolumn{3}{|c|}{ 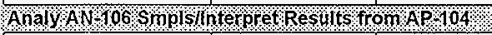 } & \multirow{2}{*}{\multicolumn{2}{|c|}{$\begin{array}{l}\text { Analy AN-106 Smpls/lnterpret Results from AP-104 } \\
0\end{array}$}} \\
\hline $150 . M 55$ & O2JAN07* & 21FEB07 & & \\
\hline \multicolumn{3}{|c|}{ 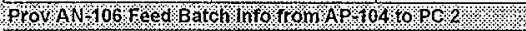 } & \multirow{2}{*}{\multicolumn{2}{|c|}{ Prov AN-106 Feed Batch info from AP-104 to PC 2}} \\
\hline $150 . \mathrm{M} 60$ & $22 F E B 07$ & OBMAR07 & & \\
\hline \multicolumn{3}{|c|}{ 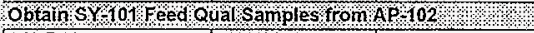 } & \multirow{2}{*}{\multicolumn{2}{|c|}{ Obtain SY-101 Feed Qual Samples from AP-102 }} \\
\hline $150 . R 10$ & 10MAY07* & 18JUN07 & & \\
\hline \multicolumn{3}{|c|}{ ProvidesY 101 Samples rom AR 102 to PC 1 , } & \multirow{2}{*}{\multicolumn{2}{|c|}{ Provide SY-101-Samples from AP-102 to PC 1}} \\
\hline $150 . R 15$ & $19 \mathrm{JUN} 07^{\star}$ & $27 \mathrm{JUN0}$ & & \\
\hline \multicolumn{3}{|c|}{ 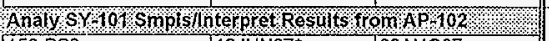 } & \multirow{2}{*}{\multicolumn{2}{|c|}{ Analy SY-101 Smpls/Interpret Results from AP-102 }} \\
\hline $150 . R 20$ & 19JUN07* & O9AUG07 & & \\
\hline \multicolumn{3}{|c|}{ ProvsY 101 red Batch nito from AP 102 to PG 1} & \multirow{2}{*}{\multicolumn{2}{|c|}{ Prov SY-101 Feed Batch info from AP-102 to PC 1}} \\
\hline $150 . R 25$ & 10AUG07 & $24 A \cup G 07$ & & \\
\hline obtain SY 101 & Guals Samples & $\mathrm{AP} / 04$ & & \\
\hline $150 . R 45$ & 13JULO ${ }^{\star}$ & 20AUGD7 & & Obtain SY-101 Feed Qual Samples from AP-104 \\
\hline Provide $S Y$ I01 & esfrom AP & 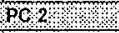 & & \\
\hline $150 . R 50$ & $21 \mathrm{AUGO}^{*}$ & 29AUGO7 & . & Provide SY-101 Samples from AP-104 to PC 2 \\
\hline
\end{tabular}




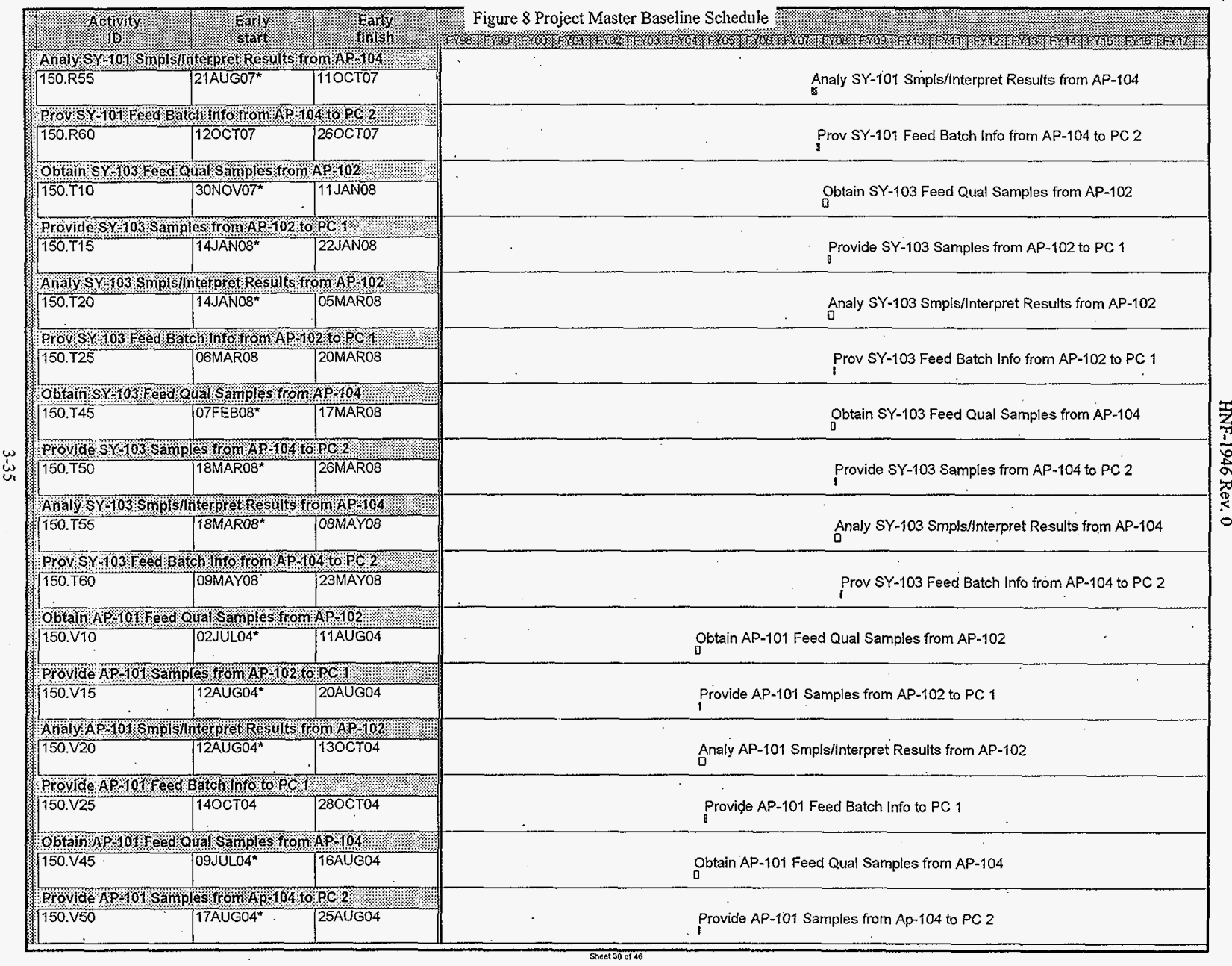




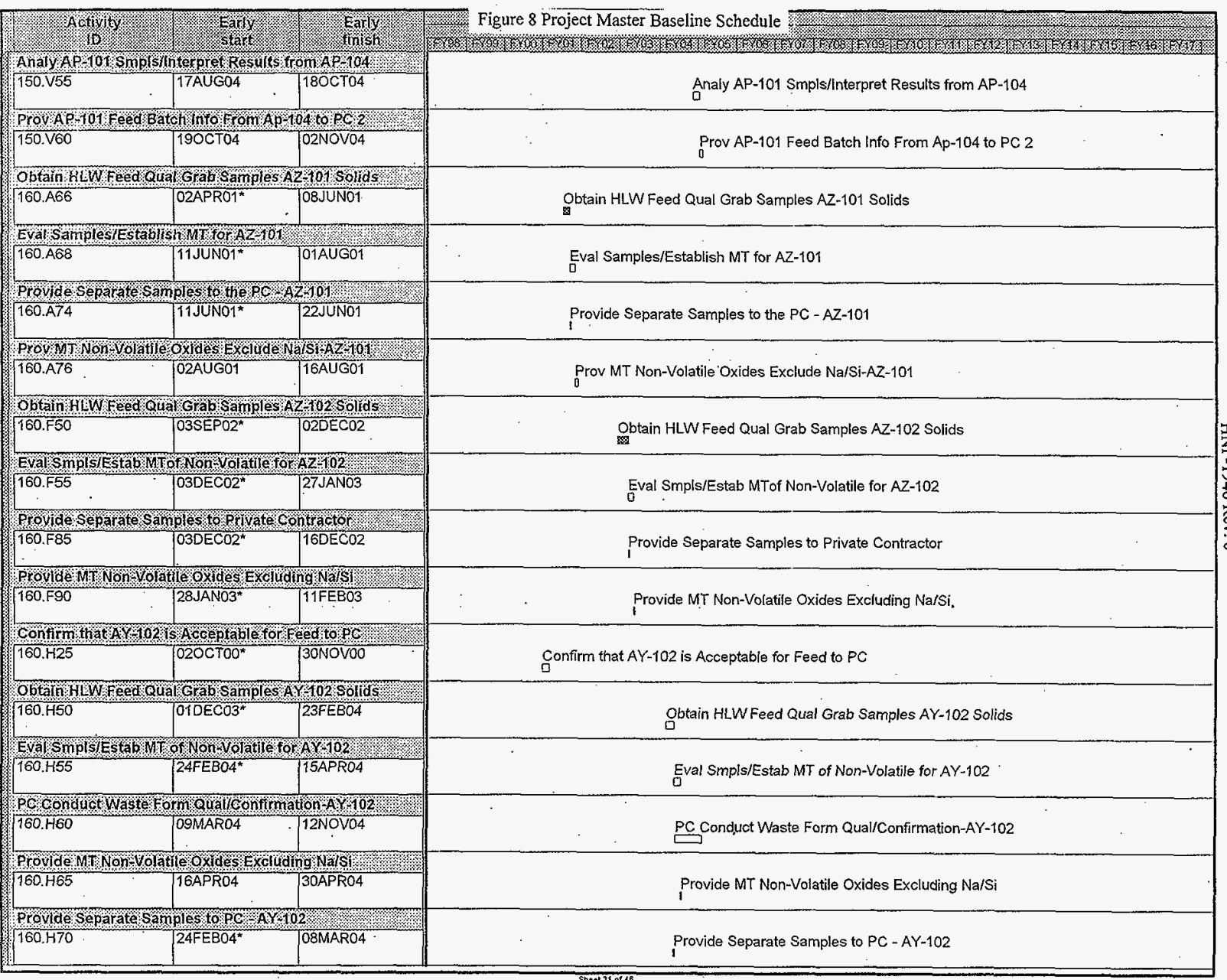




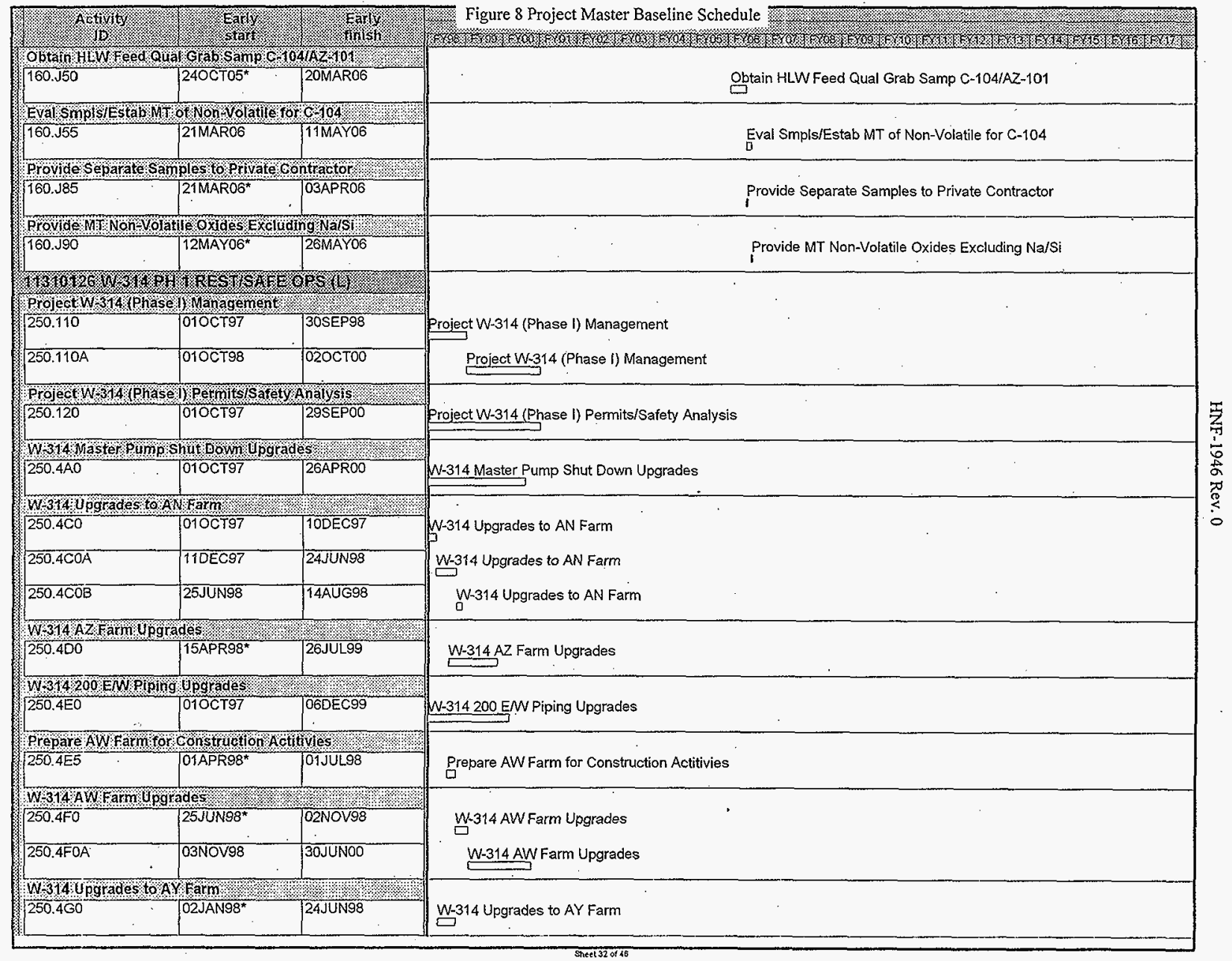




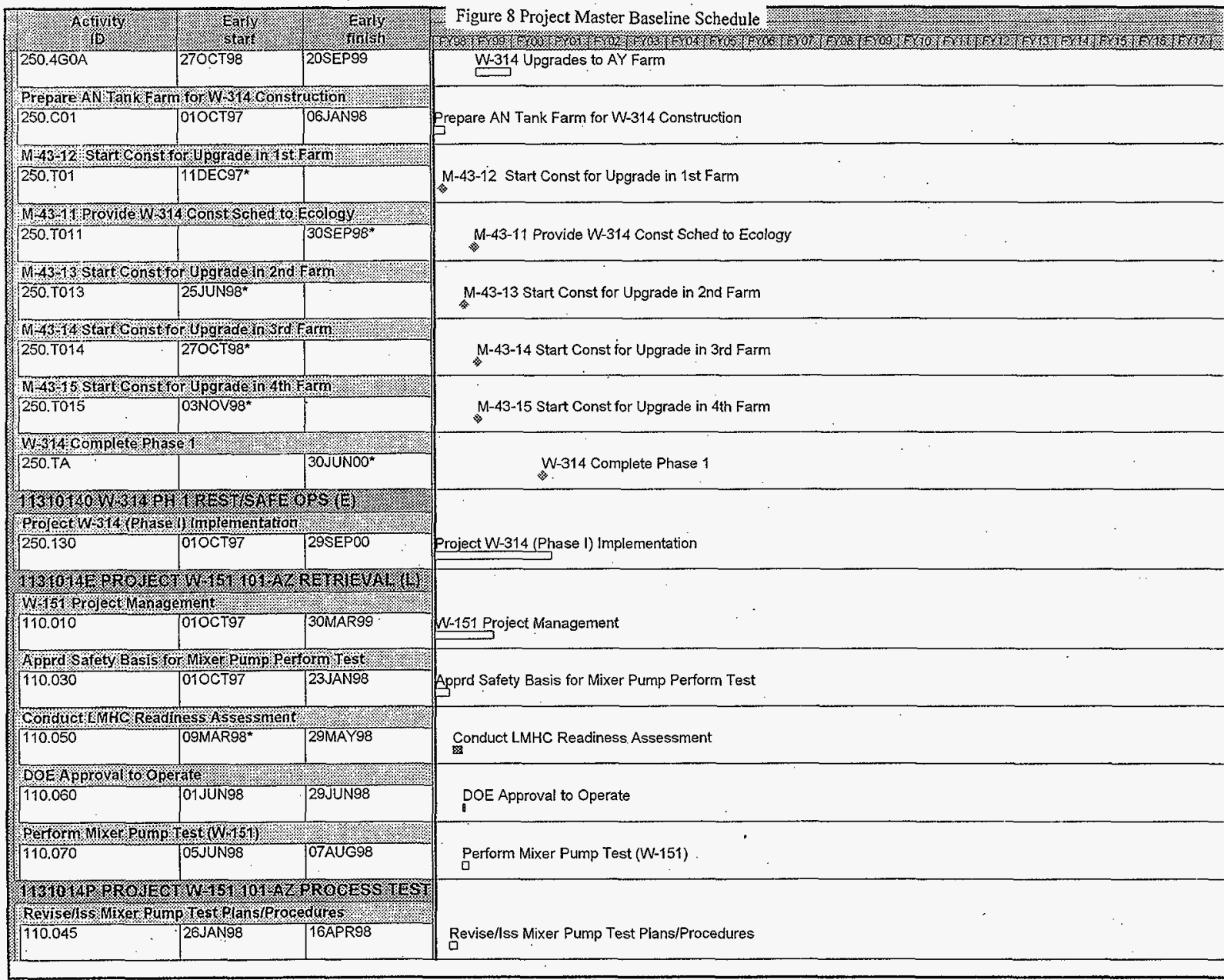




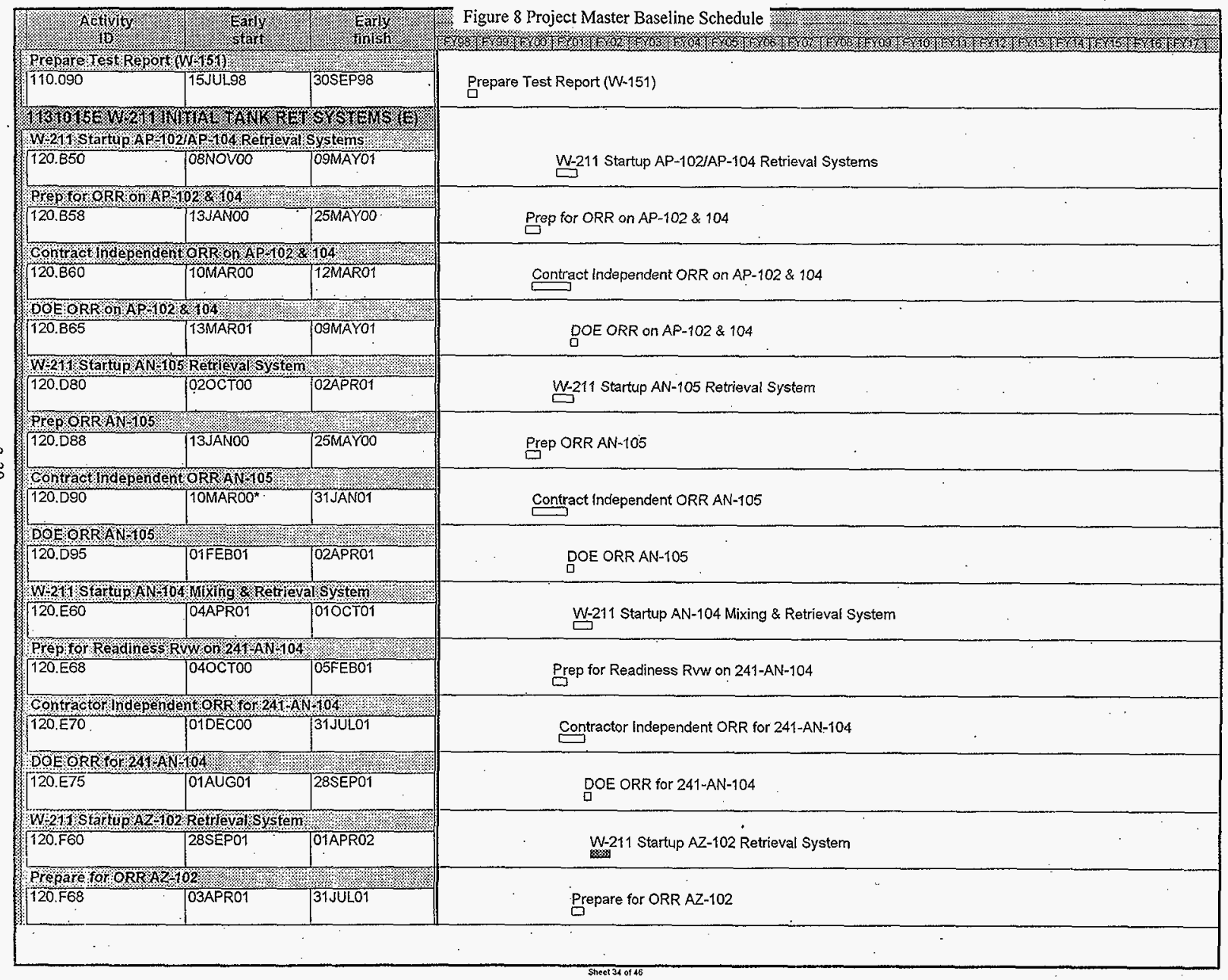




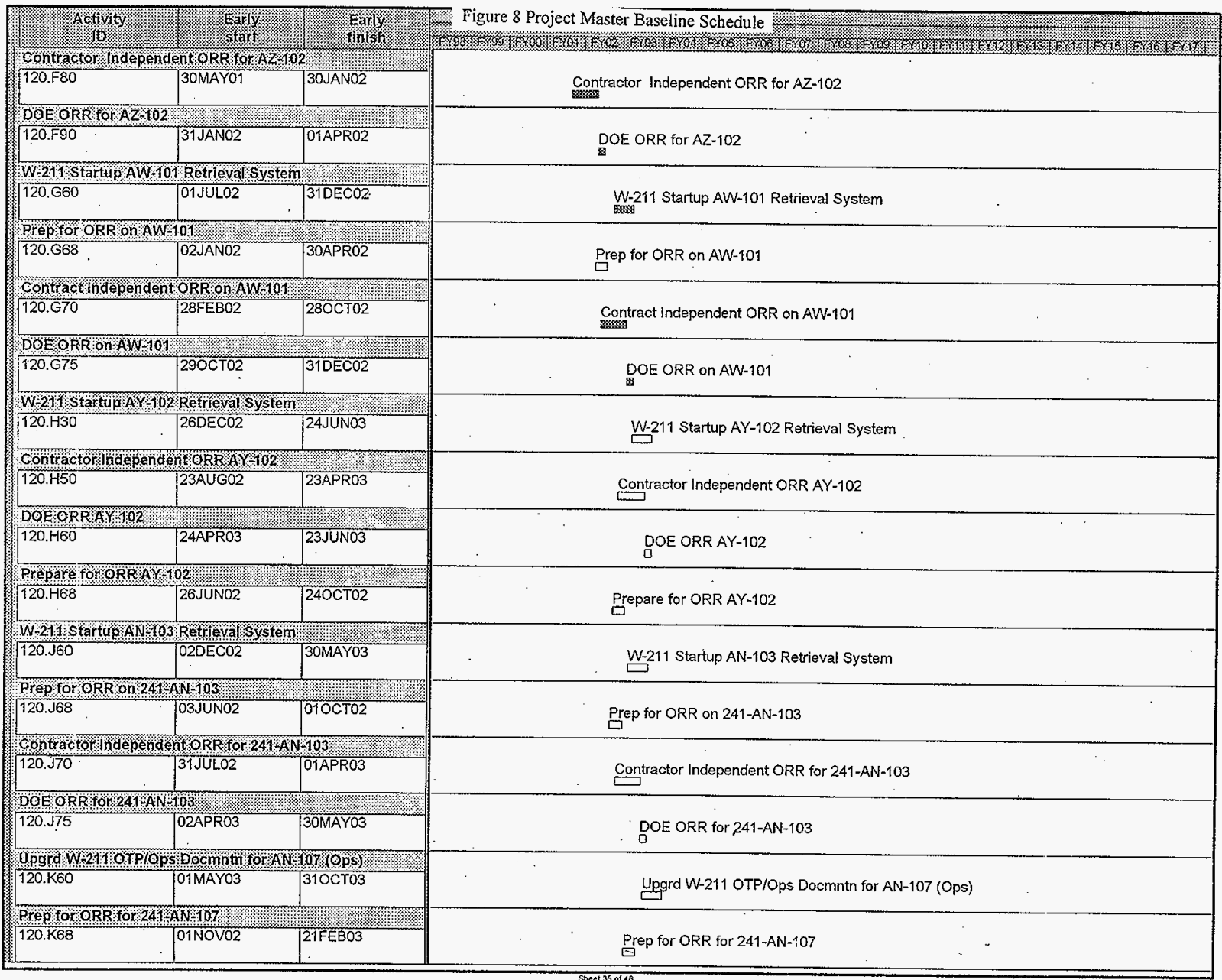




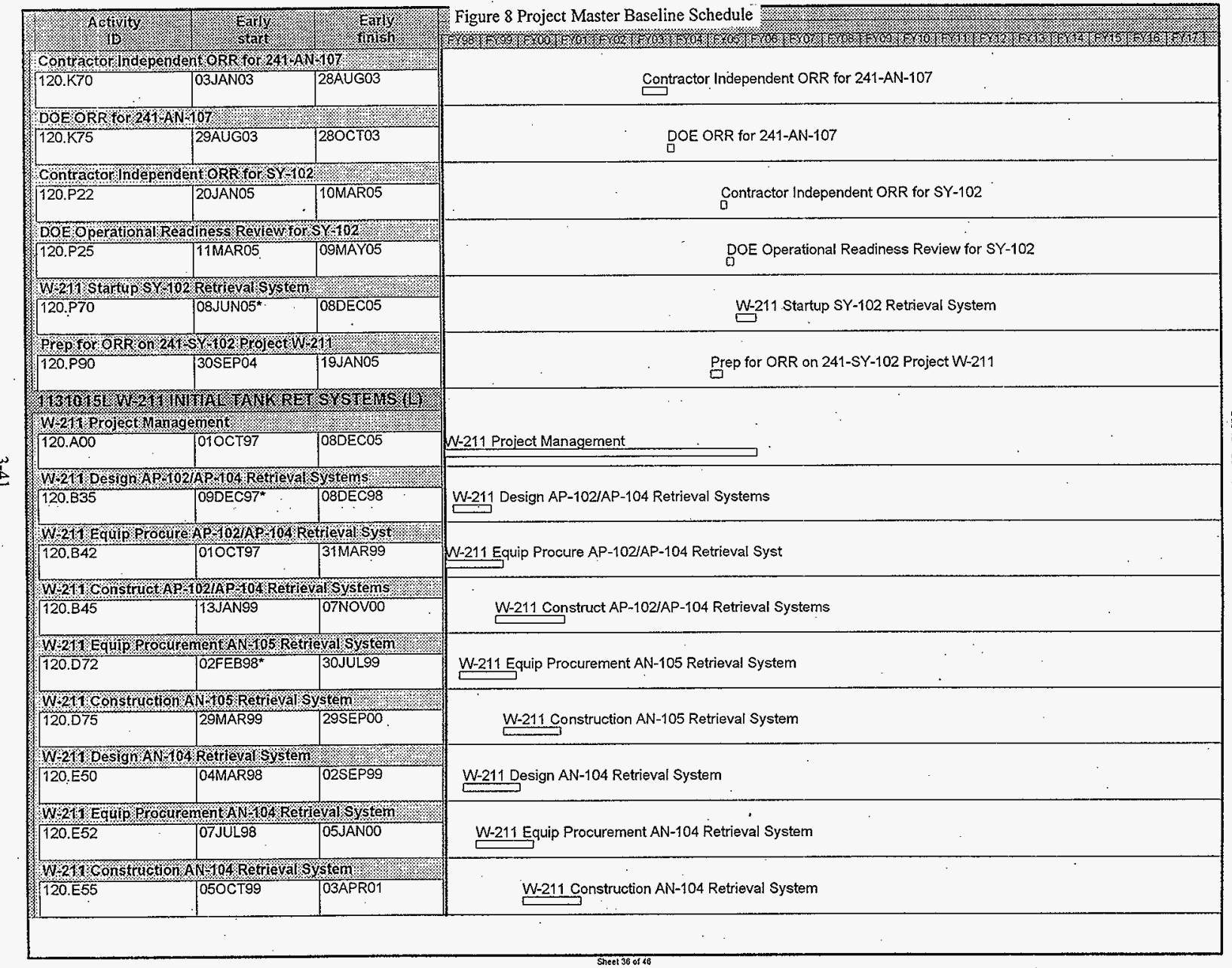




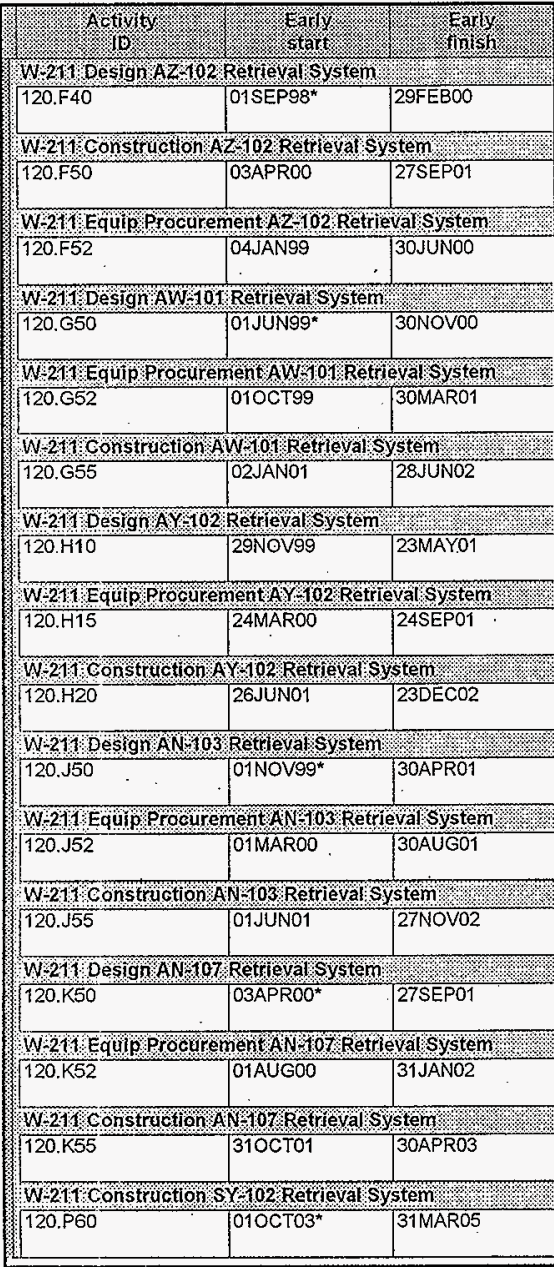

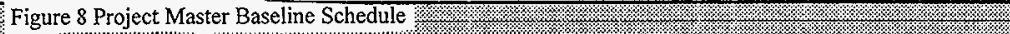
-

\section{W-211 Design AZ-102 Retrieval System}

W-211. Construction AZ-102 Retrieval System

W-211 Equip Procurement AZ-102 Retrieval System

W-211 Design AW-101 Retrieval System

W-211 Equip Procurement AW-101 Retrieval System

W-211 Construction AW-101 Retrieval System

W-211 Design AY-102 Retrieval System

W-211 Equip Procurement AY-102 Retrieval System

W-211 Construction AY-102 Retrieval System

W-211 Design AN-103 Retrieval System

W-211 Equip Procurement AN-103 Retrieval System

W-211 Construction AN-103 Retrieval System

W-211 Design AN-107 Retrieval System

W-211 Equip Procurement AN-107 Retrieval System

W-211 Construction AN-107 Retrieval System

W-211 Construction SY-102 Retrieval System 


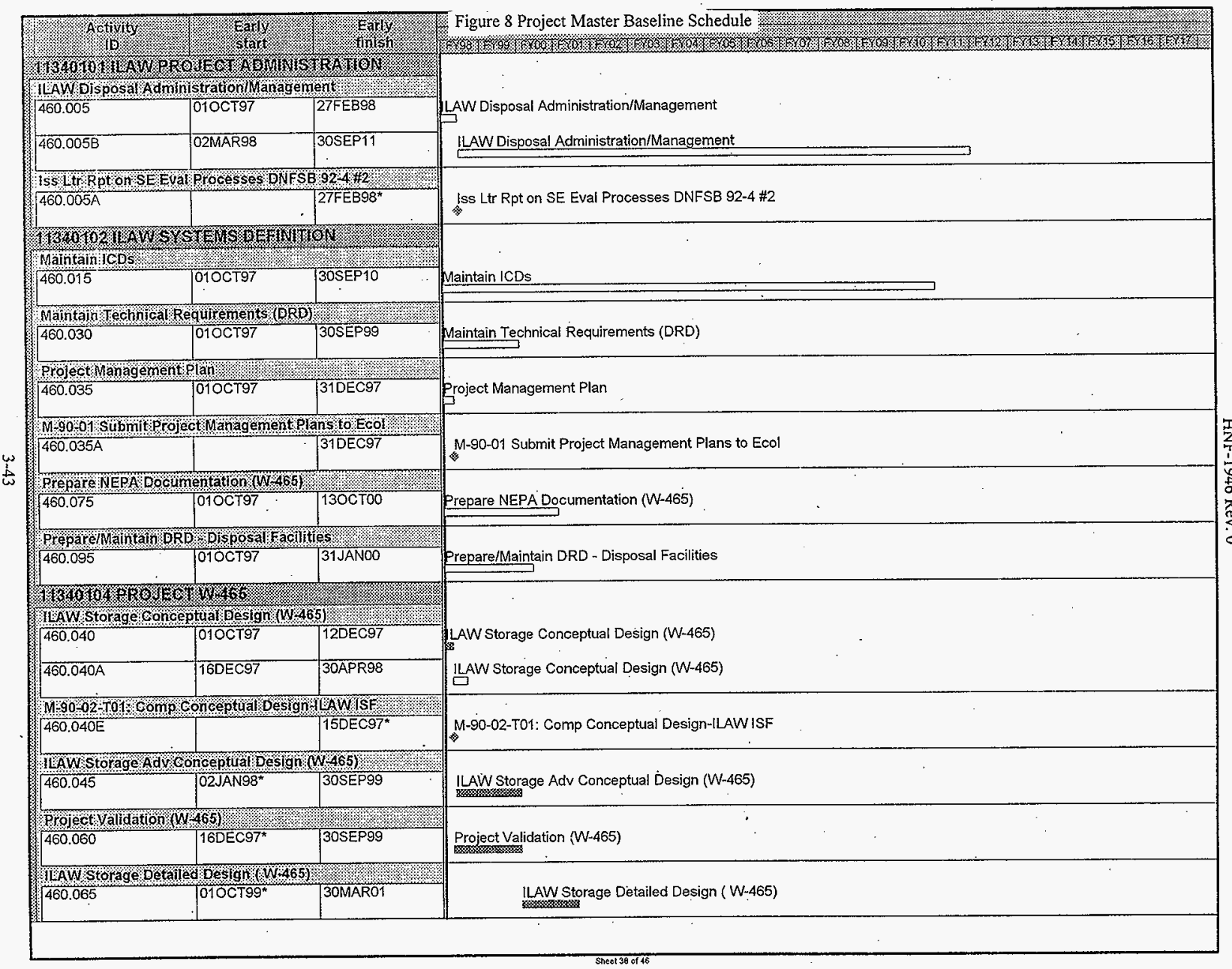




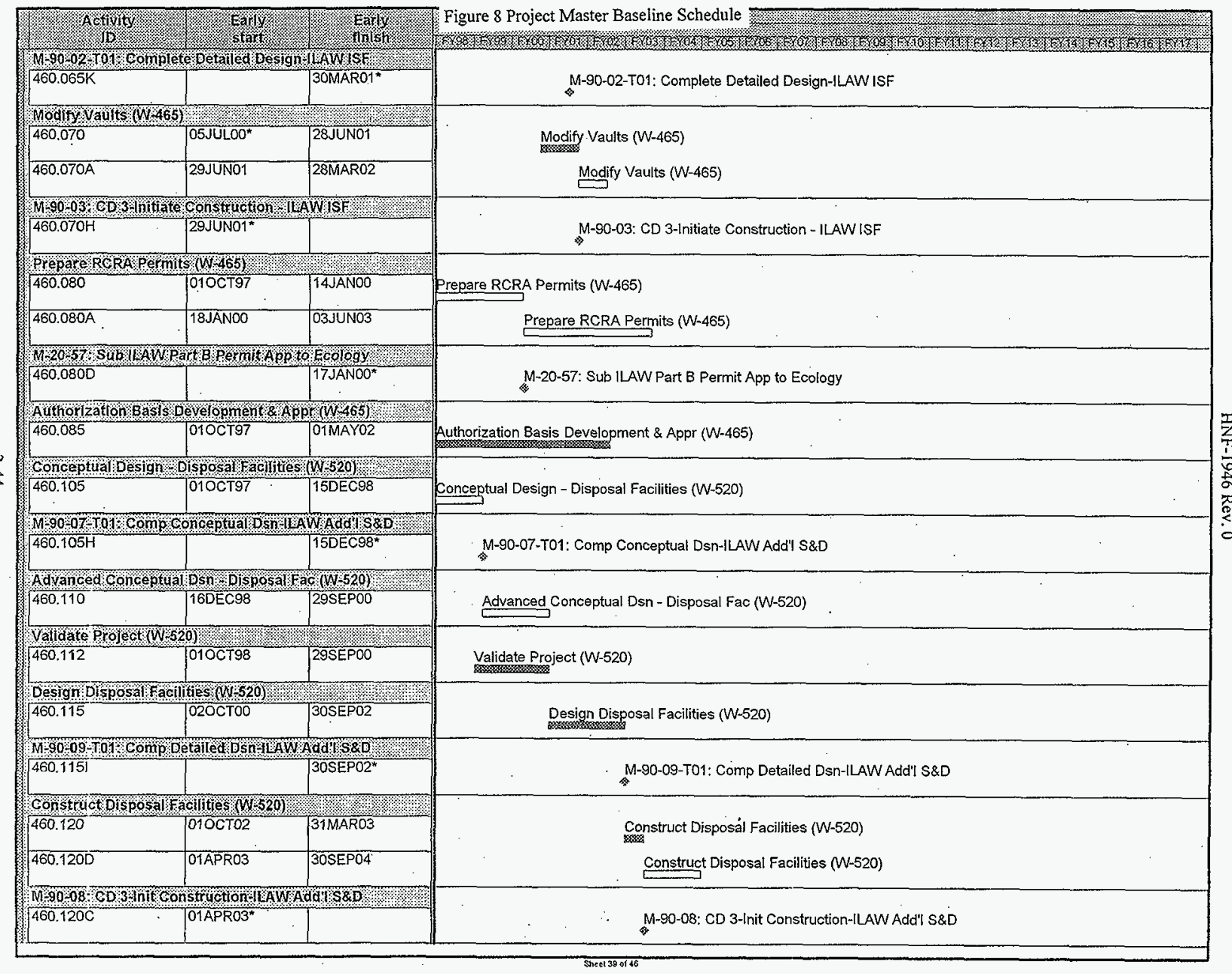




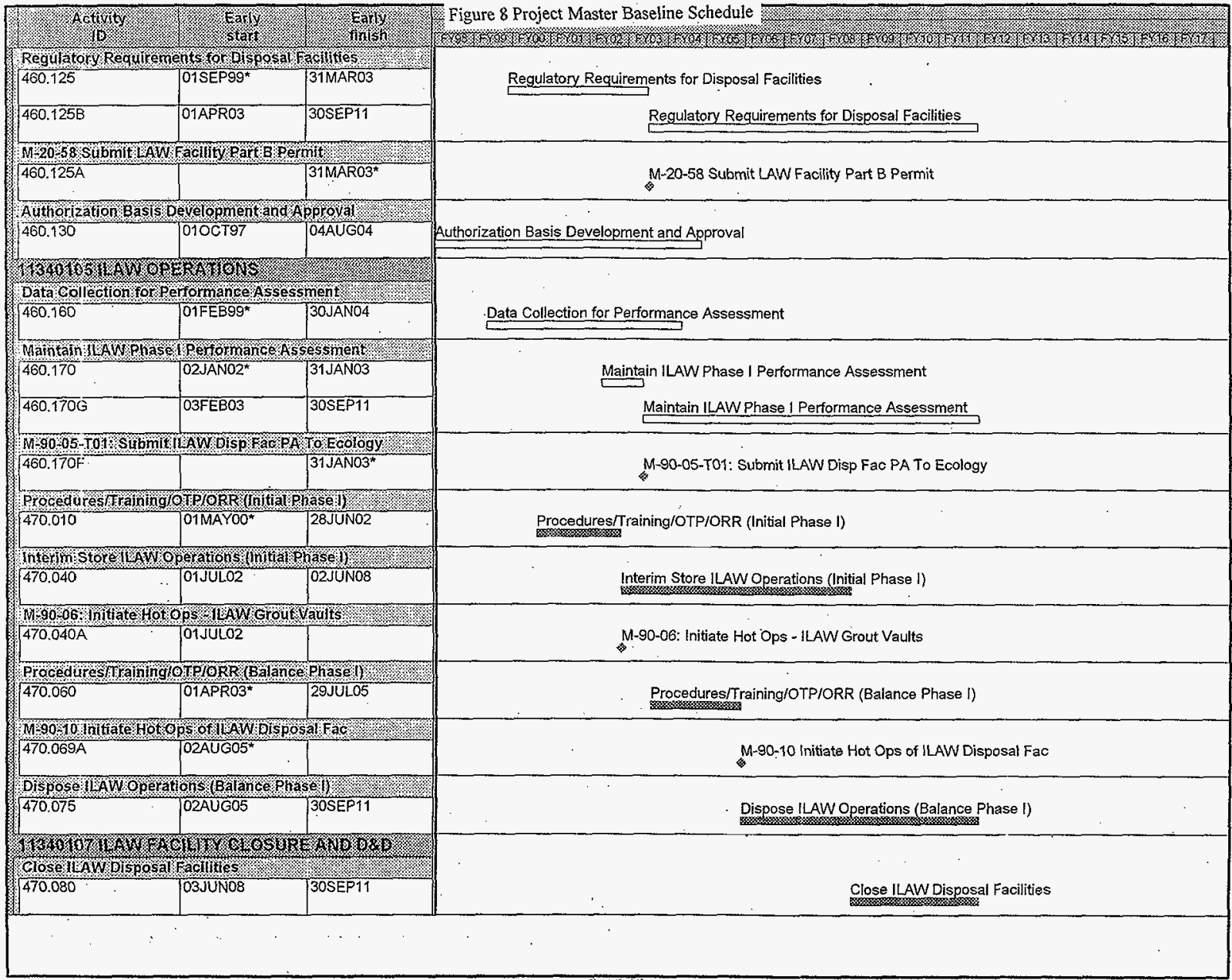




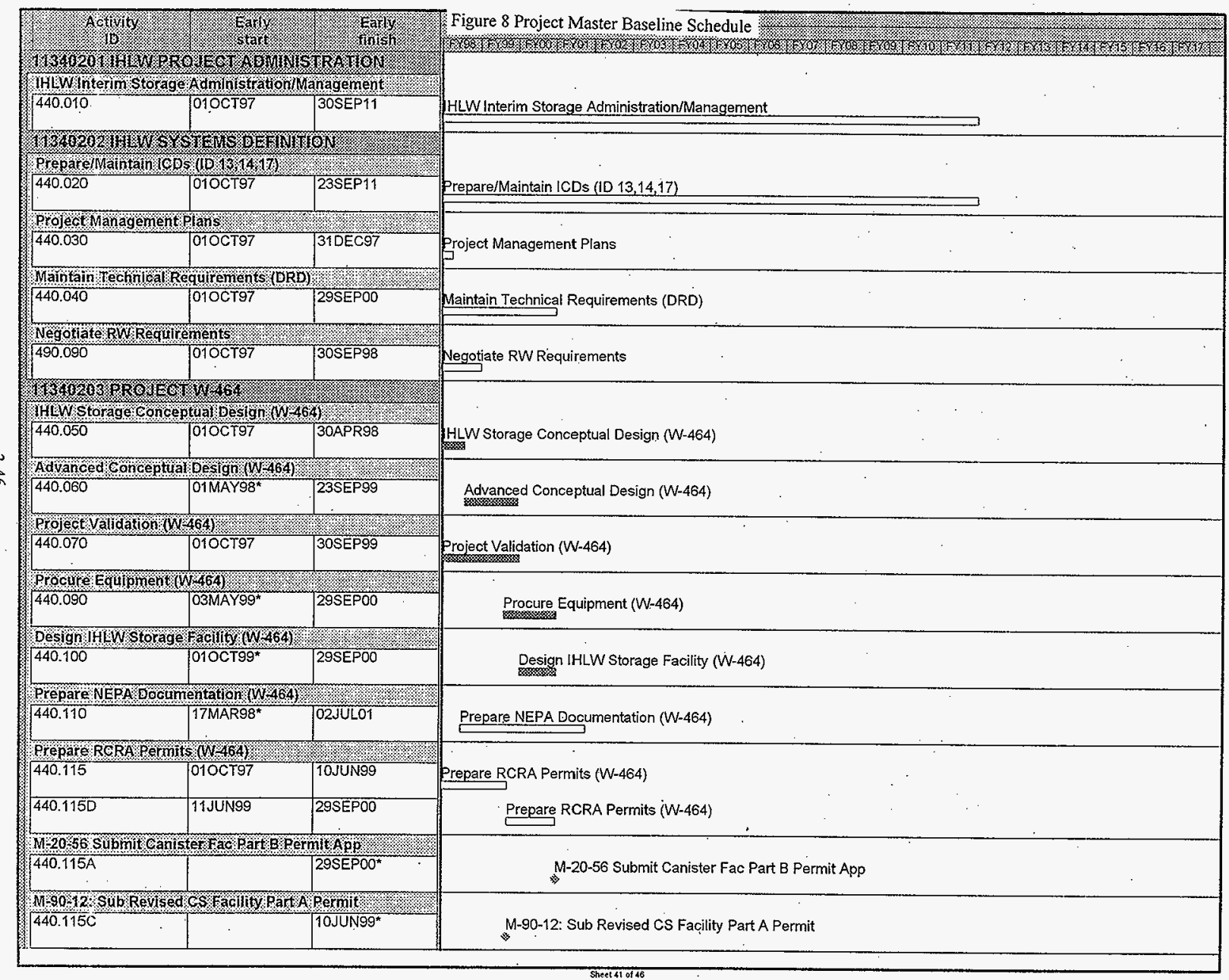




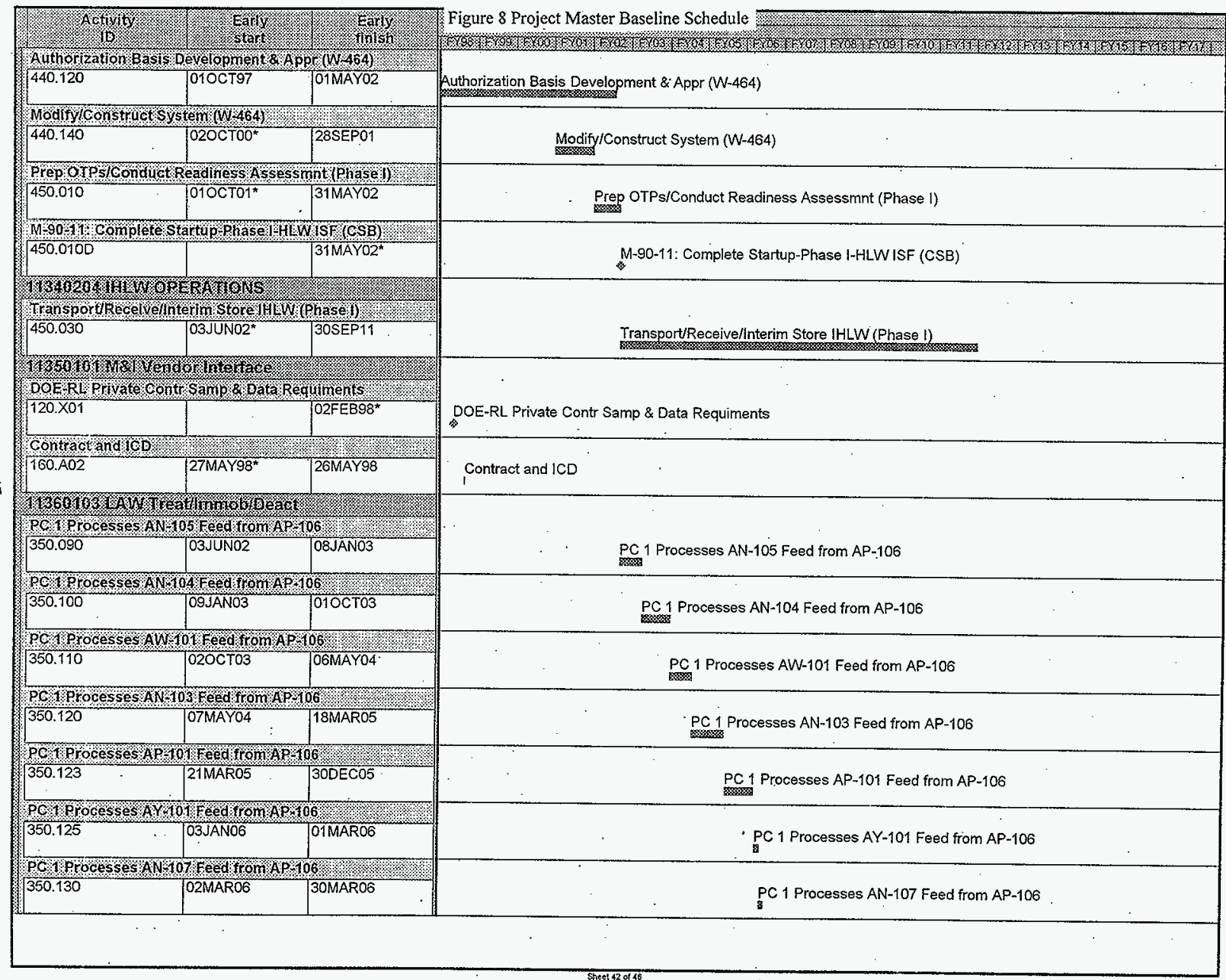




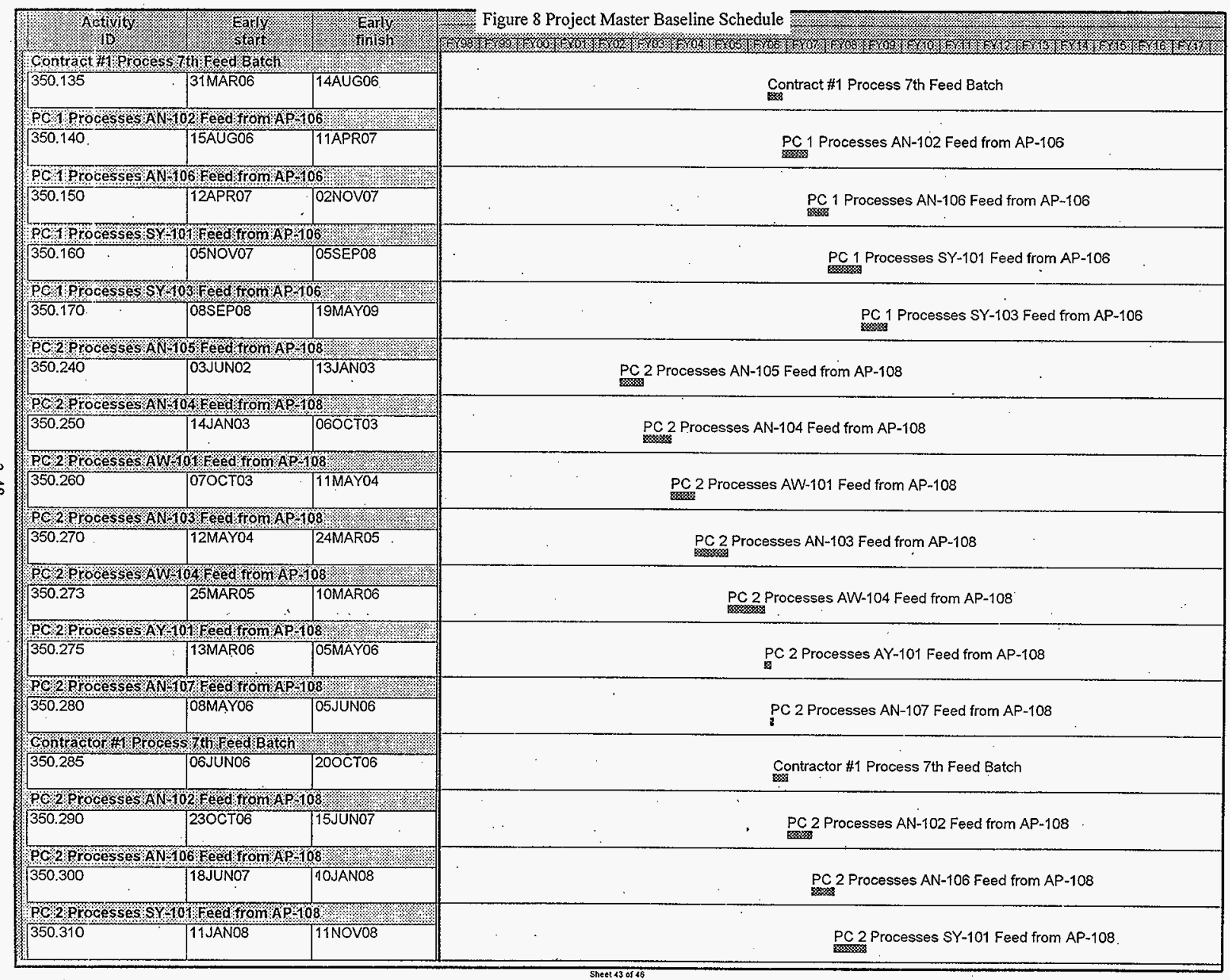




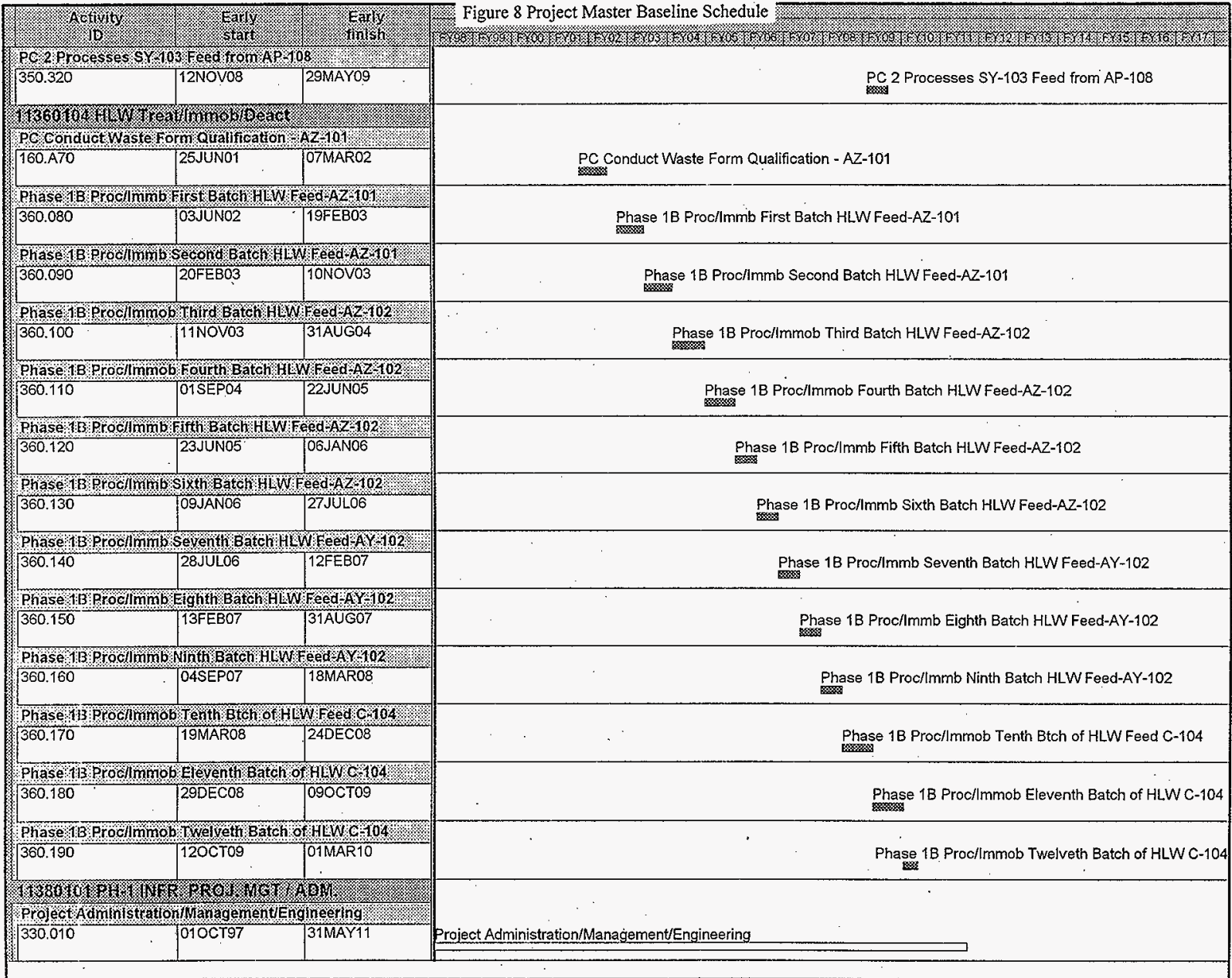




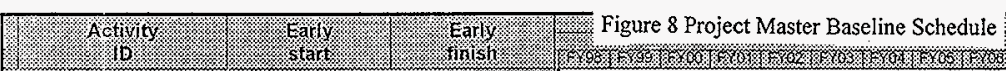

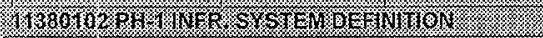

Ready PHMC OpenMánt Doc AP - OB T Tónover

\begin{tabular}{|l|l|l|l|}
\hline 150.866 & Ready PHMC Oper/Maint Doc-AP-108 Turnover \\
\hline
\end{tabular}

Ready PHMC OderiMain Dor DP 106 Tutrover

\begin{tabular}{|l|l|l|l|l|l}
\hline $150 . B 76$ & 27 MAY98* & 13 NOV98
\end{tabular}

Prep AP-106 to Turover to P ivate Contracto

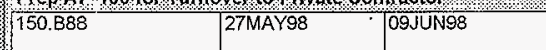

Estabilishinterface Requnits for Tan Wurnover

\begin{tabular}{|l|l|l|}
\hline $150 . \mathrm{B} 94$ & $010 \mathrm{CT} 97$ & 23JAN98 \\
\hline
\end{tabular}

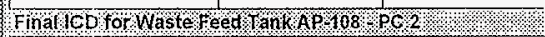

\begin{tabular}{l|l|l}
$150 . \mathrm{Bg6}^{2}$ & $27 \mathrm{MAY98}^{*}$ & 26.MAY98
\end{tabular}

Prep 1 P 108 for Turnover o Private Contractor

\begin{tabular}{|l|l|l}
\hline 150.898 & 27 MAY98 & 11 JUN98
\end{tabular}

Systems Definition .

\begin{tabular}{|l|l|l}
\hline 330.020 & 2910CT97 & 29SEPOO..
\end{tabular}

Ready PHMC Oper/Maint Doc-AP-106 Turnover

1.

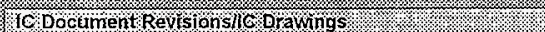

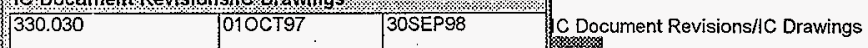

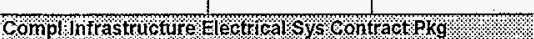

\begin{tabular}{|l|l|}
\hline 330.040 & 17 AUG98
\end{tabular}

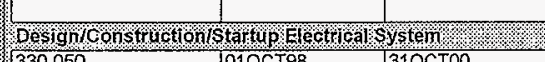

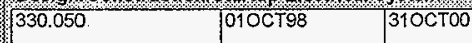

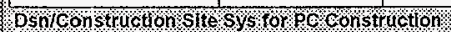

\begin{tabular}{|l|l|l:}
\hline 330.060 & $0100 T 98$ & 310 EC99
\end{tabular}

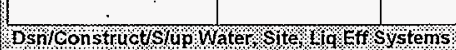

\begin{tabular}{|l|l|l|l|}
\hline 330.070 & 0100 T98 & 30MAR01
\end{tabular}

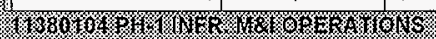

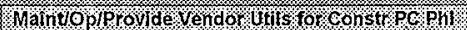

\begin{tabular}{|l|l|l}
\hline 330.080 & $04 J A N O 0$ & $310 E C 01$
\end{tabular}

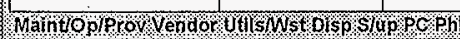

\begin{tabular}{|l|l|l|}
\hline 330.090 & O2JANO2 & 31MAYO2
\end{tabular}

Prep AP-106 for Turnover to Private Contractor

Establish Interface Reqmnts for Tank Turnover

Final ICD for Waste Feed Tank AP-108 - PC 2

Prep AP-108 for Turnover to Private Contractor

Systems Definition

Compl Infrastructure Electrical Sys Contract Pkg

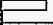

Design/Construction/Startup Electrical System

Dsn/Construction Site Sys for PC Construction

Driscos

Dsn/Construct/S/up Water, Site, Liq Eff Systems

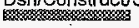

Maint/Op/Provide Vendor Utils for 'Constr PC Phl

Maint/Op/Prov Vendor Utils $/$ /st Disp S/up PC Phl

Main 


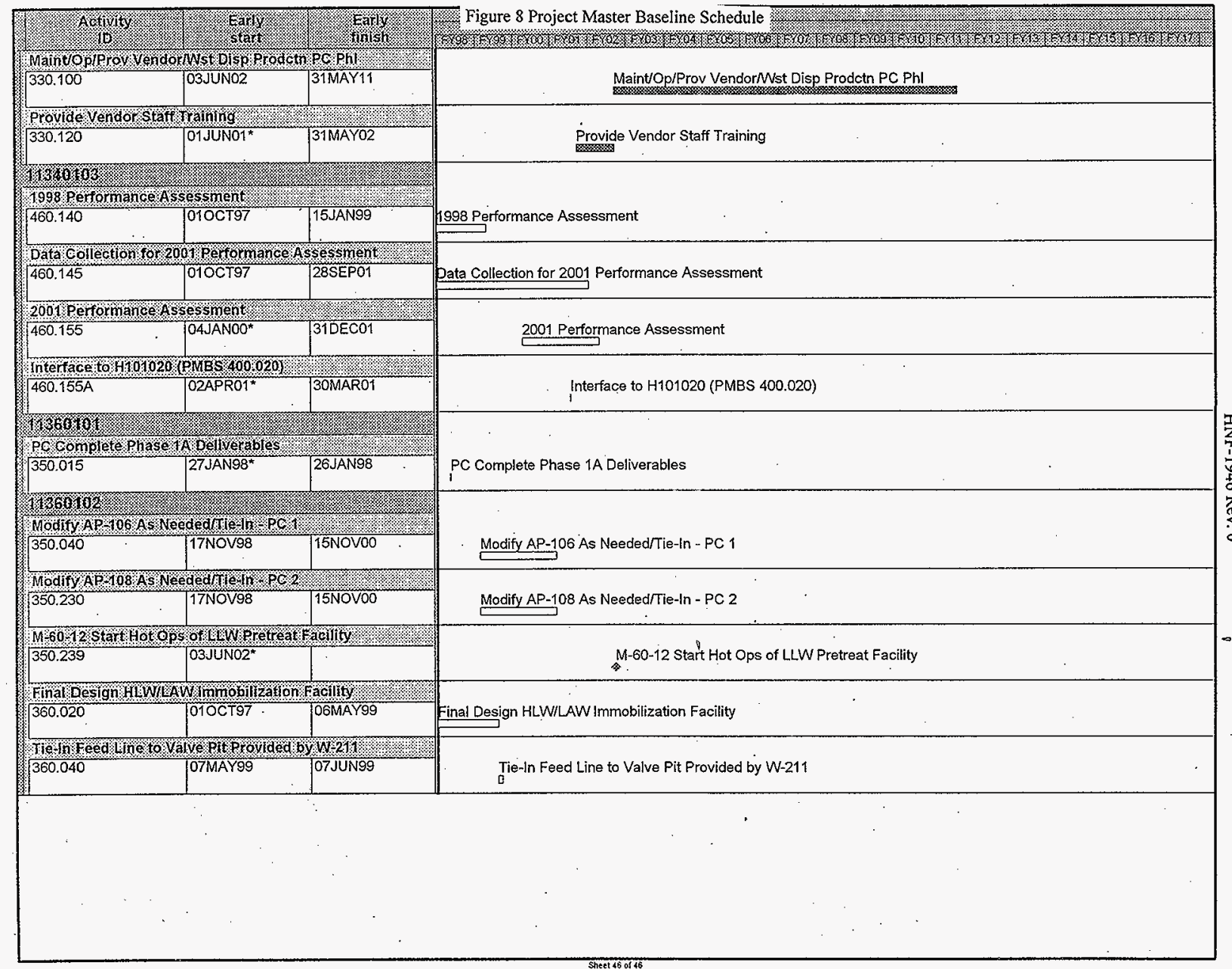


Milestones Level 1-5 : RTP vs TPA vs MYWP Due Dates

\begin{tabular}{|c|c|c|c|c|c|c|c|}
\hline $\begin{array}{l}\text { WBS } \\
\text { Level } 6\end{array}$ & $\begin{array}{l}\text { Activity } \\
\text { ID }\end{array}$ & Description & $\begin{array}{l}\text { Milestone } \\
\text { Number }\end{array}$ & $\begin{array}{l}\text { Milestone } \\
\text { Type }\end{array}$ & $\begin{array}{l}\text { RTP } \\
\text { Dates }\end{array}$ & $\begin{array}{l}\text { TPA } \\
\text { Dates }\end{array}$ & $\begin{array}{c}\text { MYWP } \\
\text { Detail } \\
\text { Dates }\end{array}$ \\
\hline 11310106 & 05079A & M-45-03A Initiate Sluicing Retrieval of C-106 & T04-98-161 & 2 & 04-Sep-98 & $31-$ Oct-97 & $30-$ Sep-98 \\
\hline 11310106 & 05079B & Action for DNFSB 93-5, 5.4.3.6.C & T04-98-161 & 5 & 04-Sep-98 & N/A & 30-Sep-98 \\
\hline 11310106 & $05079 \mathrm{C}$ & Start Retrieval Secretary Sfty Initiative W-320 & T04-98-161 & 4 & 04-Sep-98 & N/A & $30-\operatorname{Sep}-98$ \\
\hline 11310102 & 15B22E1A & M-45-02D Sub Annual SST Ret Seg Doc to Ecology & T04-99-241 & 2 & $30-$ Sep-99 & $30-$ Sep-99 & 30-Sep-99 \\
\hline 11310102 & 15B22EA & M-45-02C Sub Annual SST Ret Seg Doc to Ecology & T04-98-241 & 2 & 30-Sep-98 & $30-$ Sep- 98 & $30-\operatorname{Sep}-98$ \\
\hline 11310126 & $25 \mathrm{~T} 011$ & M-43-11 Provide W-314 Const Sched to Ecology & T03-98-054 & 2 & 30-Sep-98 & 30-Sep-98 & $30-$ Sep-98 \\
\hline 11310126 & $25 \mathrm{~T} 012$ & M-43-12 Start Const for Upgrade in 1st Farm & T03-99-055 & 2 & 11-Dec-97 & $30-5 u n-99$ & 30-Jun-99 \\
\hline 11310126 & $25 \mathrm{~T} 013$ & M-43-13 Start Const for Upgrade in 2nd Farm & T03-00-056 & 2 & 25-Jun-98 & 30-Jun-00 & $30-J u n-00$ \\
\hline 11310126 & $25 \mathrm{T014}$ & M-43-14 Start Const for Upgrade in 3rd Farm & T03-01-057 & 2 & $27-0 c t-98$ & 31-Mar-01 & 31-Mar-01 \\
\hline 11310126 & $25 \mathrm{~T} 015$ & M-43-15 Start Const for Upgrade in 4th Farm & T03-02-058 & 2 & 03-Nov-98 & $31-\mathrm{Mar}-02$ & 29-Mar-02 \\
\hline 11360102 & $35239 \mathrm{~A}$ & M-60-12 Start Hot Ops of LLW Pretreat Facility & T06-02-131 & 2 & 03-Jun-02 & 31-Dec-02 & 03-Jun-02 \\
\hline 11340203 & $44115 \mathrm{Cl}$ & M-90-12: Sub Revised CS Facility Part A Permit & T09-99-100 & 2 & 10-Jun-99 & 30-Jun-99 & 01-Jun-99 \\
\hline 11340203 & $44115 \mathrm{D} 9 \mathrm{M}$ & M-20-56: Sub CSB Part B Application to Ecology & T09-01-100 & 2 & 29-Sep-00 & 31-Dec-00 & 29-Sep-00 \\
\hline 11340203 & $45010 \mathrm{D}$ & M-90-11: Complete Startup-Phase Y-HLW ISF (CSB) & T09-03-003 & 2 & 31-May-02 & 31-Dec-02 & 31-May-02 \\
\hline 11340101 & $46005 \mathrm{~A} 7$ & Iss Ltr Rpt on SE Eval Processes DNFSB 92-4 \#2 & T09-98-101 & 4 & $27-F e b-98$ & N/A & N/A \\
\hline 11340102 & 46035AM & M-90-01: Sub Project Management Plan to Ecology & T09-98-100 & 2 & 31-Dec-97 & 31-Dec-97 & 31-Dec-97 \\
\hline 11340104 & $46040 \mathrm{E}$ & M-90-02-T01: Comp Conceptual Design-ILAW ISF & T09-98-022 & 3 & 15-Dec-97 & 15-Dec-97 & 15-Dec-97 \\
\hline 11340104 & $46065 \mathrm{~K}$ & M-90-04-T01: Complete Detailed Design - ILAW ISF & T09-01-010 & 3 & 30-Mar-01 & 30-Jun-01 & 29-Jun-01 \\
\hline 11340104 & $46070 \mathrm{H}$ & M-90-03: CD 3-Initiate Construction - ILAW ISF & T09-00-003 & 2 & 29-Jun-01 & 30-Jun-01 & 29-Jun-01 \\
\hline 11340104 & 46080D8 & M-20-57: Sub ILAW Part B Permit App to Ecology & T09-01-021 & 2 & 17-Jan-00 & 31-Dec-00 & 17-Jan-00 \\
\hline 11340104 & $46105 \mathrm{H}$ & M-90-07-T01: Comp Conceptual Dsn-ILAW Add'I S\& & T09-99-011 & 3 & 15-Dec-98 & 30-Jun-00 & 15-Dec-98 \\
\hline 11340104 & 461151 & M-90-09-T01: Comp Detailed Dsn - ILAW Add'I S\&D & T09-04-001 & 3 & 30-Sep-02 & 31-Mar-03 & $30-$ Sep- 02 \\
\hline 11340104 & $46120 \mathrm{C}$ & M-90-08: CD 3-Init Construction-ILAW Add'l S\&D & T09-05-005 & 2 & 0I-Apr-03 & 30-Jun-03 & $01-A p r-03$ \\
\hline 11340104 & $46125 \mathrm{H}$ & M-20-58: Sub ILAW Disp Part B Permit App to Ecol & T09-01-020 & 2 & 31-Mar-03 & 31-Dec-03 & 31-Mar-03 \\
\hline 11340105 & $46170 \mathrm{~F}$ & M-90-05-T01: Submit ILAW Disp Fac PA To Ecology & y T09-03-101 & 3 & 31-Jan-03 & 31-Dec-01 & 31-Jan-03 \\
\hline 11340105 & $47040 \mathrm{~A}$ & M-90-06: Initiate Hot Ops - ILAW Grout Vaults & T09-02-002 & 2 & 01-Jul-02 & 31-Dec-02 & 04-Jun-02 \\
\hline 11340105 & $47069 \mathrm{CM}$ & M-90-10: Initiate Hot Operations - ILAW Module I & T09-06-100 & 2 & 02-Aug-05 & 31-Dec-05 & 02-Aug-05 \\
\hline
\end{tabular}

Milestone Type

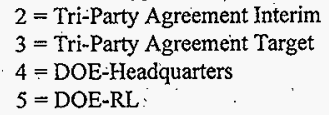


HNF-1946 Rev. 0

Table 2 Milestone Comparison

List of Terms

$\begin{array}{ll}\text { CSB } & \text { Canister Storage Building } \\ \text { DNFSB } & \text { Defense Nuclear Facilities Safety Board } \\ \text { HLW } & \text { high-level waste } \\ \text { ILAW } & \text { immobilized low-activity waste } \\ \text { ISF } & \text { Interim Storage Facility } \\ \text { LLW } & \text { low-level waste } \\ \text { MYWP } & \text { Multi-Year Work Plan } \\ \text { PA } & \text { Performance Assessment } \\ \text { RTP } & \text { Readiness-to-Proceed } \\ \text { S\&D } & \text { Storage and Disposal } \\ \text { SST } & \text { single-shell tank } \\ \text { TPA } & \text { Tri-Party Agreement }\end{array}$




\section{0 $\operatorname{CosT}$}

The TWRS Retrieval and Disposal and other supporting TWRS estimates and costs have been updated to reflect the changes that resulted during the RTP development phase. The cost products are discussed in the following sections and are included as tables in this section.

\subsection{SUMMARY COST SPREADSHEET}

The cost spreadsheet (Table 3) summarizes the preliminary TWRS dollars, by fiscal year (FY 1998 through FY 2011), necessary to perform the following activities:

- TWRS Retrieval and Disposal Program for Phase 1B scope based on the revised RTP planning

- TWRS Phase 1B scope required to support the TWRS Retrieval and Disposal mission

- $\quad$ Scope Transfer Shortfalls identified since submittal of the TWRS FY 1998 MYWP for activities that are transitioned to TWRS Retrieval and Disposal from elsewhere in TWRS.

A complete summary and analysis of TWRS Project costs, including those shown in this document related to the Retrieval and Disposal Phase $1 \mathrm{~B}$ mission, are included in the Financial Analysis (Wells 1998).

\subsection{DESCRIPTION OF THE TYPES OF COSTS}

This section describes the types of costs which are included in the Initial Updated Baseline as shown on Table 3, Summary Cost Spreadsheet.

The TWRS Retrieval and Disposal Program costs for Phase 1B Logics are the estimates for Phase 1 work defined by the Level 1 Logics for Waste Feed Delivery (LAW and HLW), Privatization Infrastructure, and Immobilized Waste. Technical Basis Review packages were prepared to document these costs as part of the RTP process. The values include escalation through FY 2006 and are in constant year dollars (FY 2006) thereafter.

Other TWRS Retrieval and Disposal Program costs for Phase 1B support scope (not in Logics) include estimates for Project Management and Administration, Feed Process System 
HNF-1946 Rev. 0

This page intentionally left blank. 
HNF-1946 Rev. 0

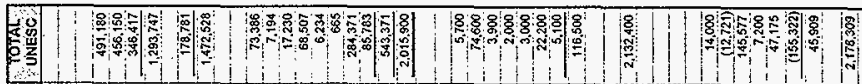

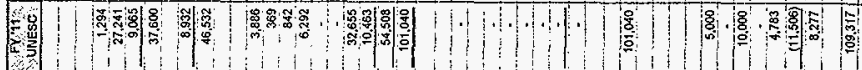

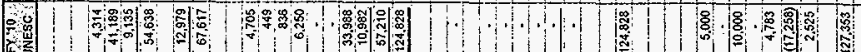
5: 17:

3.

3. Fong

8. (N)

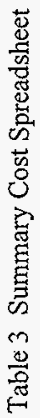

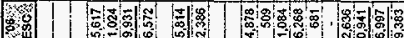

4

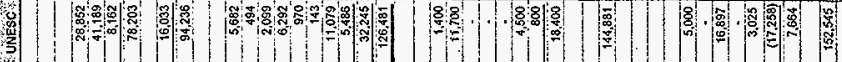

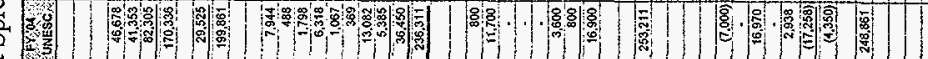

8

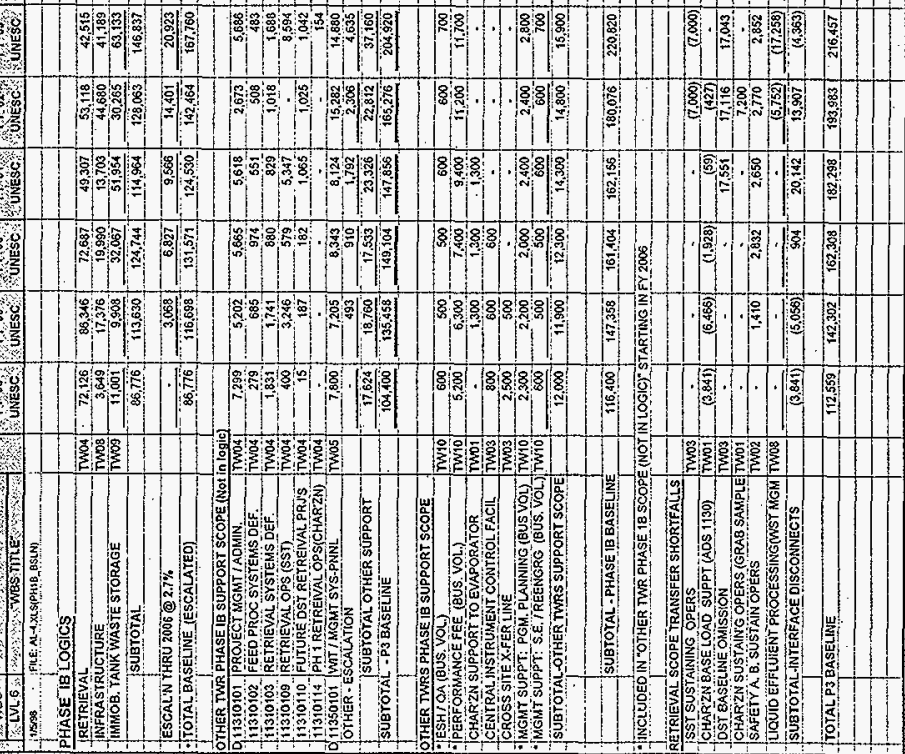


HNF-1946 Rev. 0

This page intentionally left blank. 
Definition, Retrieval System Definition, Retrieval Operations (Single-Shell Tank), Future Double-Shell Tank Retrieval Projects, Phase 1 Retrieval Operations (Characterization), and . Process Waste Support costs associated with Management and Integration Contractor Vendor Interface and Phase 1 Program Management by the RL/Pacific Northwest National Laboratory Waste Integration Team. These activities and costs are essential to the Retrieval and Disposal Program, but were not covered or partially covered by activities in the Level 1 Logics. The costs used are from the TWRS FY 1998 MYWP (Lenseigne 1997) with escalation through FY 2006 and constant year dollars (FY 2006) thereafter.

Other TWRS Phase 1B Support Scope includes the costs for a pro-rata share of the TWRS cross-cutting activities and other miscellaneous activities (funded elsewhere in the TWRS) that are connected to and necessary to support the TWRS Retrieval and Disposal Program as defined by the RTP assessment. Activities include Environmental, Safety and Health/Quality Assurance management and oversight based on a business volume share; Performance Fee based on a business volume share; Characterization support to the evaporator; Central Instrument Control Facility; the Cross-Site Transfer Line; management support for program planning; and Systems Engineering and reengineering, based on a business volume share. The cost numbers used are from the TWRS FY 1998 MYWP (Lenseigne 1997) with escalation through FY 2006 and constant year dollars (FY 2006) thereafter.

Scope Transfer Shortfalls are cost disconnects that have been identified during RTP and since the development of the TWRS FY 1998 MYWP (Lenseigne 1997) for activities that will be transitioned to the TWRS Retrieval and Disposal Program. Although these activities are not specific to the Phase 1 Level 1 Logics, additional resources are required to fund these activities. They include single-shell tank sustaining operation, Characterization base load support, doubleshell tank baseline omission, Characterization sustaining operations, Safety Authorization Basis (AB) sustaining operations, and liquid effluent processing. Liquid effluent processing costs are included in the "Phase 1B Logics" baseline and are deducted here as it has been determined that this work is funded by the Waste Management Program. These cost numbers include escalation through FY 2006 and constant year dollars (FY 2006) thereafter.

A detailed discussion and analysis of these costs are included in the Financial Analysis (Wells 1998).

\subsection{COST-LOADING REPORT}

The cost-loading report (Table 4) summarizes the TWRS Retrieval and Disposal costs by fiscal year (FY 1998 through FY 2011) necessary to perform work defined by the Level 1 Logics and the Project Master Baseline Schedule. The dollars are unescalated. These are the same costs on Table 3 as "Phase 1B Logics."

Table 4 provides a summary by Level 6 WBS element followed by the additional details of Level 1 Logic activities. Pricing of resources to generate these costs was done in the 
HNF-1946 Rev. 0

Primavera Systems, Inc. scheduling software using input from the TBR packages' CEIS's and approved labor rate tables. 
Table 4. Cost Loading Report

REPORT DATE 29DEC97

PRIMAVERA PROJECT PLANNER

$16: 30$

COST LOADING REPORT

RTP 17DEC97

RTP cOST (SK) by WOS Level

TOTAL USAGE FOR YEAR

DATA DATE OTOCTIT

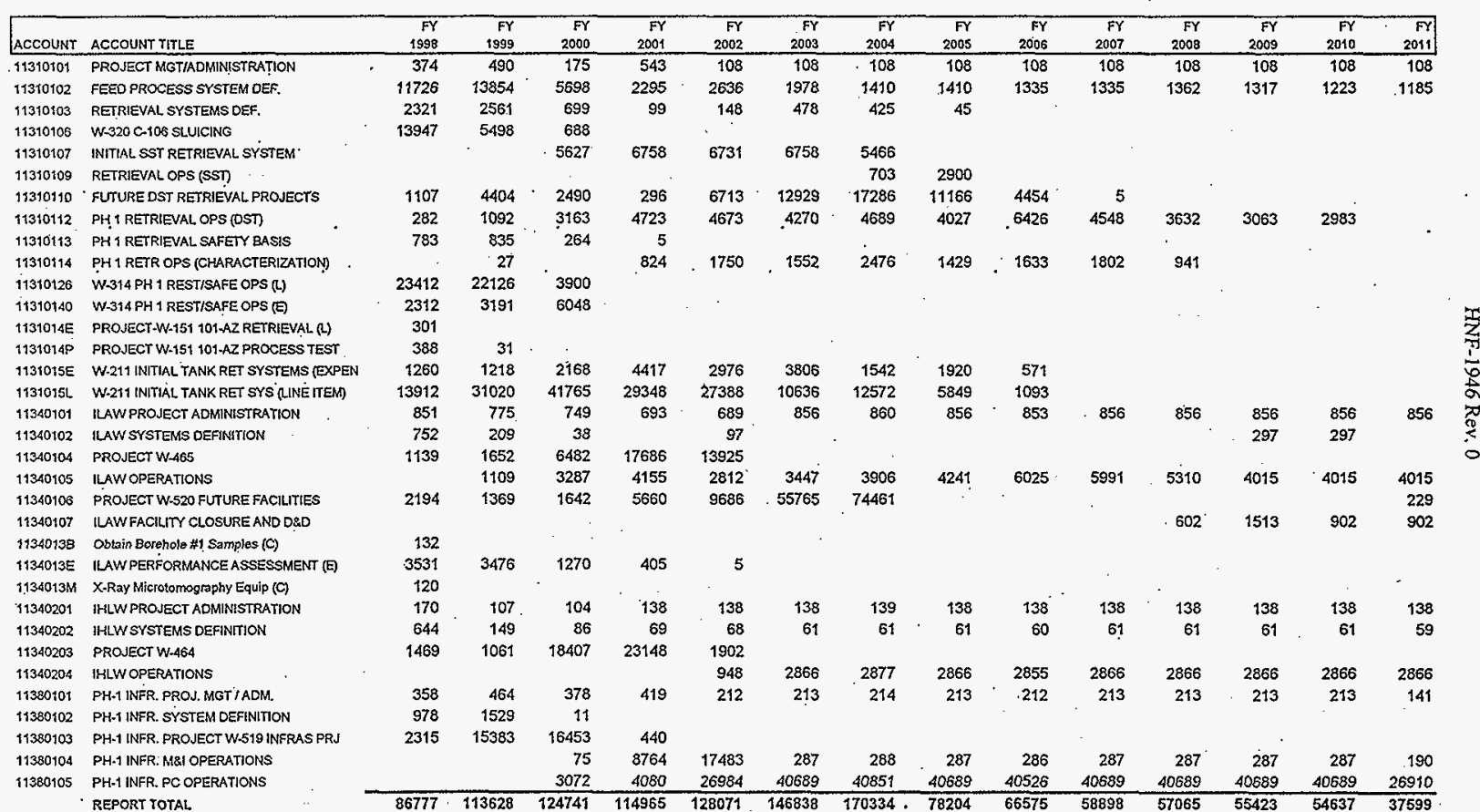


Table 4. Cost Loading Report

\begin{tabular}{|c|c|c|c|}
\hline $\begin{array}{l}\text { REPORT DATE 29DEC97 } \\
\{6: 45\end{array}$ & $\cdot$ & $\begin{array}{l}\text { PRIMAAVERA PROJECT PLANNER } \\
\text { COST LOADING REPORT }\end{array}$ & RTP 17DEC97 \\
\hline RTP COST (\$K) by WBS Level 6 by LeVel 7 & $\therefore$ & TOTAL USAGE FOR YEAR & DATA DATE 010CT97 \\
\hline
\end{tabular}

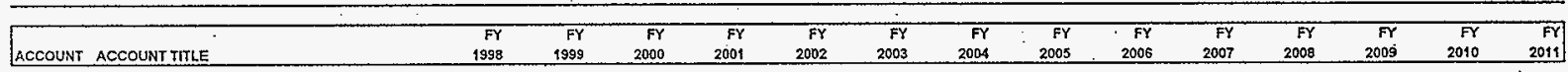

11310101 PROJECT MGT/ADMINISTRATION -

130.865 TWRS Management Assessment (LAW)
130.870 Prepare Plan/Document for Mgmt Assessment (LAW)

160.A13 Piepare Plar/Document for Mgmt Assessment (HLW)

160.A18 TWis Management Assessment (HLW)

620.095 Dov/ss Draft WFD Tech Baselino Summary

620.097 Update WFD Technical Easeline Summary

TOTAL 11310101

11310102 FEED PROCESS SYSTEM DEF.

110.047 Ped Mixer Pump Test Sampling \& Analysis

$120 . c 05$ Obtain AY-101 Samplos

A $120 . c 10$ Characterize AY-101

do $120 . C 12$ ReviewAY-101 Historical Data

120.C14 Establish Dilution Ramts for AY-101 Process Tést

120.015 Confirm Plans and Requirements for AY-101

120.C20 Updato BB| \& TCR For TankAY-101

120.D07 Dovelop LAW Confirm Tank T Meets Batch XD00

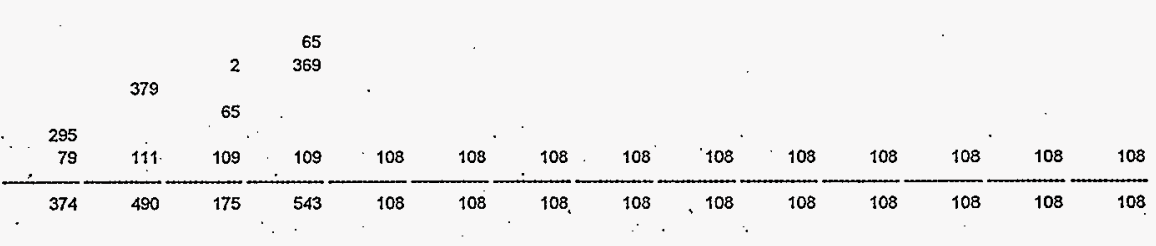

120.D08 Develop Waste Transter to PC 020

120.009 Dovelop in Tank Processing Contrïl DQ0O

120.010 Evaluate AN-105 Samples for Adequato Matls

120.D11 Develop DQO: PH 1 Tank Wasta Transfer Cọntol

120.012 Develop HLWWaste Delivery toPC DCO

120.015 RuwaN-105 Historical Data/Cont if Add'l Info Rq

120.D20 - Estab Dilution Reqmnts/Diss Behav 10: AN-106

120.025 Characterize AN-105

120.030 Update Best Basis inventory and TCR for AN-105

120.035 Perf Max Implied Radionuclide Concentrate Study

120.040 Confirm Plans and Requirements for AN-105

120.E05 Evaluato AN-104 Samples for Adequato Matts

120.E10 Estab Oilution ReqmntefOiss Behay for AN-104

120.E15 ReviewAN-104 Historical Data

120.E20 Characterize AN-104

120.E25 Update Best Basis Inventory and TCR for AN-104

120.E35 Confirm Plans and Requirements for AN-104

253

253

$\begin{array}{ccc}2 & 24 & 402 \\ 15 & \\ 12 & 59 \\ & & 24 \\ & & 26\end{array}$

\begin{tabular}{|c|c|c|}
\hline \multirow{4}{*}{2} & & \\
\hline & 24 & 402 \\
\hline & 15 & \\
\hline & 12 & 59 \\
\hline & & $\begin{array}{l}24 \\
26\end{array}$ \\
\hline
\end{tabular}

120.605. Evaluate AW.101 Samplos Adequato Matts 
Table 4. Cost Loading Report

REPORT DATE 29DEC97

RIMAVERA PROJEGT PLANN

RTP 17DEC97

$$
\text { 16:45 }
$$

COST LOADING REPORT

RTP COST (SK) by WBS Level 6 by Level 7

TOTAL USAGE FOR YEAR

DAIA DATE $010 \mathrm{OGT}$

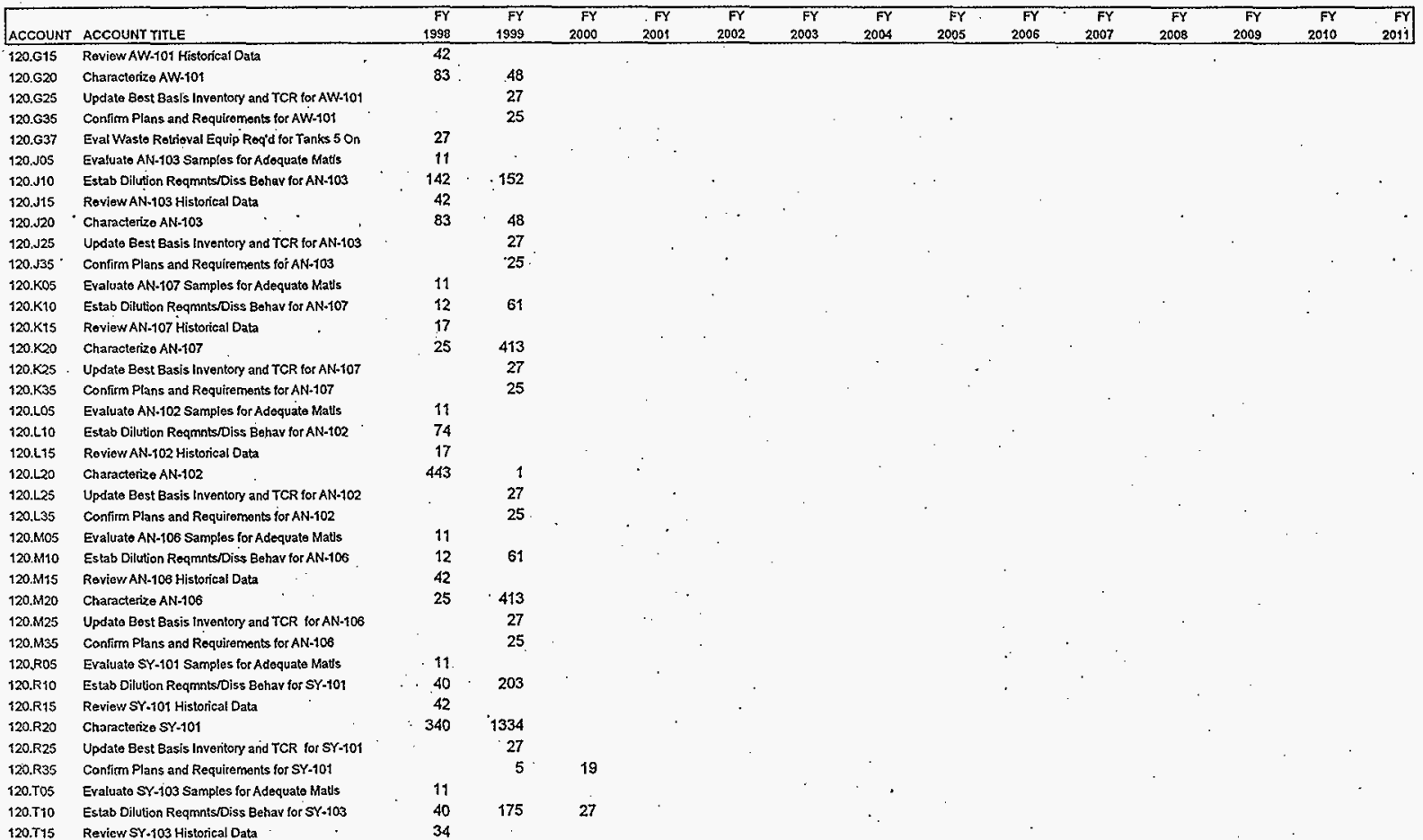


Table 4. Cost Loading Report

PRIMAVERA PROJECT PLANNER COST LOADING REPORT

REPORT DATE $290 E C 97$

TOTAL USAGE FOR YEAR

DATA DATE $010 \mathrm{CT} 97$

RTP COST (SK) by WBS Level 6 by Level 7

\begin{tabular}{|c|c|c|c|c|c|c|c|c|c|c|c|c|c|c|}
\hline ACCOUNT AGCOUNT TITLE & $\begin{array}{r}F Y \\
9998\end{array}$ & $\begin{array}{r}F Y \\
1999\end{array}$ & $\begin{array}{r}F Y \\
2000\end{array}$ & $\begin{array}{r}F Y \\
2001\end{array}$ & $\begin{array}{r}F Y \\
2002\end{array}$ & $\begin{array}{r}F Y \\
2003\end{array}$ & $\begin{array}{r}F Y \\
2004\end{array}$ & $\begin{array}{r}F Y \\
2005\end{array}$ & $\begin{array}{r}F Y \\
200 S\end{array}$ & $\begin{array}{r}F Y \\
2007\end{array}$ & $\begin{array}{r}F Y \\
2008\end{array}$ & $\begin{array}{r}F Y \\
2009\end{array}$ & $\begin{array}{r}F Y \\
2010\end{array}$ & $\begin{array}{r}F Y \\
\end{array}$ \\
\hline
\end{tabular}

$120 . T 25$ Update Best Basis laventory and TCR for SY 103 ,
$120 . T 35$ Confirm Plans and Requirements for SY. 103

120.v05 Obtain AP-101 Samples

120.V10 Characterizo.AP-101

120.V12 ReviewAP-101 Historieal Data

120.V14 Establish Dilution Rqmts for AP-101 Process Test

120.V15 Confirm Plans and Requirements for AP-101 . .

120.V20 Update BBI \& TCR For Tank AP-10t

120. WD5 Obtain AW.104 Samples . ' 11

120.W10 Characterizo AW-104

120.W12 Roview AW:104 Fistorical Data

120.W14 Establish Dilution Rqmts for AW-104 Process Test

120.W15 Confirm Fians and Requirements for AW:104

120,W20 Update BBI \& TCR For Tank AW-104

130.010 Phase I Tank Waste Transfer Control

i 130.E05 10 Regd Spares WFD 1st Balches LAW L LAW

$\frac{1}{5} 130.845$ Prepare for Sampling During LAW Feed Staging

130.875 Compare RAMO\&M Concept $w$ OrP Results

130,B80 Assess Sea. Waste Util \& Samp Anly Agnst Plans

130.Nso Prep for Sampling During HLW Feed Staging AZ-10

150.804 Perform Feed Rate Trado Study

150,806 Establish Feed Rates (RL. Docision).

150.BOS Maintain WFD Char Matrices, Prob Spec DQOS

150.822 Maintain Oporations Utilization Plan

150.824 Maintain ICDs.

150.882 Peform Feed Tank Fill Status Trade Study

150.884 Establish Feed Tank Fill Status (RL Decision)

160.A12 Estab Tech (Process) Reqmnts-Phase I Sidg Pret

160.A15 Assess Sec Waste, Uti Samp Anly Agst Plan (HiLW)

160.A16 Prep D00 for Privatization Char of Pot HLW Feed

$160 . A 17$ Comparo RAMOSA Concopt w OTP Rosuits (HLW)

160.A19 Retr. Reviso Protreat Rqmis (AZ-101/2 \& AY-102)

160.A20 Finalize HLW Pretreat Rqmts (AZ-101/2\& AY*102)

$160 . A 22$ Determino Appropriato Altemative Tanks

160.A43 Optimize PH1 Feed Sludge Wash by Proc Simulatio

160.A44 Deter if Existing Data Meets DOO Neods - AZ-101

160.A46 Take Additional Grab Samples from AZ-101

160.A48 Petrooc Smpl Analysis/Sidg Wash Test-AZ-101

$1 t$

$24^{\circ} 405$

17

12

11

17

$24 \quad 405$

$\begin{array}{ll}12 & 60 \\ 24\end{array}$

24
26
$515 \quad 504$

13

216

$\begin{array}{rr}515 & 504 \\ 19 & \\ 784 & 705 \\ 6 & \end{array}$

504

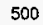

50

501

50

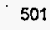

501

501

501

501

507

37

6.

85

. 4

119
1396

683.

157

4

38

40

118
880
500

115
748
482

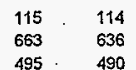

115
616
492

115
540

115
540

115
465

1.15
465

115
492

115
447

115
352

115 
Table 4. Cost Loading Report

\begin{tabular}{|c|c|c|}
\hline $\begin{array}{l}\text { REPORT DATE 29DEC97 } \\
\{6: 45\end{array}$ & $\begin{array}{l}\text { PRIMAVERA PROJECT PLANNER } \\
\text { COST LOADING REPORT }\end{array}$ & RIP $17 D E C 97$ \\
\hline RTP COST (\$K) by WBS Level 6 by LeVel 7 & TOTAL USAGE FOR YEAR & DATA DATE $010 \mathrm{CT} 97$ \\
\hline
\end{tabular}

\begin{tabular}{|c|c|c|c|c|c|c|c|c|c|c|c|c|c|c|c|}
\hline ACCOUNT & ACCOUNT TITLE & $\begin{array}{r}F Y \\
1998 \\
\end{array}$ & $\begin{array}{r}F Y \\
1999 \\
\end{array}$ & $\begin{array}{r}F Y \\
2000\end{array}$ & $\begin{array}{r}F Y \\
2009\end{array}$ & $\begin{array}{r}F Y \\
2002 \\
\end{array}$ & $\begin{array}{r}F Y \\
2003\end{array}$ & $\begin{array}{r}F Y \\
2004 \\
\end{array}$ & $\begin{array}{r}F Y \\
2005 \\
\end{array}$ & $\begin{array}{r}F Y \\
2006 \\
\end{array}$ & $\begin{array}{r}F Y \\
2007\end{array}$ & $\begin{array}{r}F Y \\
2008\end{array}$ & $\begin{array}{r}F Y \\
2009\end{array}$ & $\begin{array}{r}F Y \\
2010\end{array}$ & $\begin{array}{r}F Y \\
2011\end{array}$ \\
\hline $160 . \mathrm{F} 00$ & Deter if Existing Oata Meets DOAO Needs - AZ-102 & 27 & & & & & & & & & & & & & \\
\hline 160.Fos & Obtain Core Smpls/Prep Subsmpis/Comp for AZ-102 & 1889 & & & & & & & & & & & & & \\
\hline $160 . \mathrm{F} 10$ & PerfiDoc Smpl Analysis/SIdgWash Testing - AZ-102 & 211 & 307 & & & . & . & & & & & & & & \\
\hline $160 . \mathrm{F} 12$ & Update $B B 1$ and TCR for $A Z-102$ & & . 27 & & & & & & & 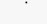 & & & & & \\
\hline $160 . F 15$ & Confirm that $A Z-102$ is Aecoptabto for Foed to PC & $\therefore$ & 25 & & & & & & & & & & & & \\
\hline 160.400 & Deter if Existing Data Meets DQO Neods - AY- 102 & 27 & & & & & & & & & & & & & \\
\hline 160.H05. & Obth Coro Smplss/Prop Subsmpls/Comp for AY-102 & 26 & 774 & 1059 & & . & & & & & & & & & . \\
\hline 160.420 & Pertidoe Smpi Analysis/Sidg Wash Tosting-AY-102 & & & 505 & & & & & & & & & & & \\
\hline $160 . \mathrm{H} 22$ & Update $\mathrm{BBI}$ and TCR for $\mathrm{AY}-102$ & & . & & 26 & . & & & . & & & & & & 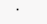 \\
\hline 160.900 & Doter if Existing Data Meets DOO Needs - C-104. & 27 & & & & & & & & & & & & & \\
\hline 160.505 & ' Obtain Core Smpls/Prep Subsmpls/Coinp for C-104 & 288 & 1585 & & & $\therefore$ & & & & & & . & & & - \\
\hline 160.510 & Perficoc Smp/ Analysis/SIdgWash Testing C-104 & 28 & 487 & & & & & & & & & & & & \\
\hline 160.212 & Update Bel and TCR for C-104 & & & 26 & & & & : & & & & & & & \\
\hline $160 . \mathrm{J} 15$ & Confirm that C-104 is Acceptable for Foed to PC & & & 24 & & & & & & & & & & & \\
\hline 620.015 & Develop Wasto Food Delivery Satety Basis DQO & 16 & & & & & & & & & & & & & \\
\hline 620.020 & Maintain Waste Feed Delivery System Summany & 1321 & 873 & 256 & 256 & 254 & 255 & 255 & 255 & 255 & 255 & 255 & 255 & 255 & 255 \\
\hline 620.055 & Develop Waste Feed Delivery Permitting DQO & 16 & & & & & & & & & & & & & \\
\hline 620.050 & Eval Enymmnt Basetine HLWhLLW Feed Dellyory & 75 & & . & & & & & & & & & & & \\
\hline 620.070 & Update Environmental Baseline & 132 & & & & & . & . & & & & . & . & & \\
\hline 620.090 & Maintain Envitonmt Baselino for Phase I Ext Ops & & 134 & 131 & 131 & .130 & & & & & . & & & & \\
\hline
\end{tabular}

TOTAL 11310102

$\begin{array}{lllllllllllll}11726 & 13854 & 5698 & 2295 & 2636 & 1978 & 1410 & 1410 & 1335 & 1335 & 1362 & 1317 & 1223\end{array}$

11310103 RETRIEVAL SYSTEMSOEF,

120.C25 Evatuato Equipment Rqmts in AY-101

120.E40 Reviso W-211 FDC for AN-104

120_G3S Deen Board/Aeqn Strategy PHI Rat After AN-104

120.639 Adjust AcQuistion Strategy WFD System

120.640 Rovise W-211 FDC Ior AW-101

120.440 Rovise W-211 FOC for AN-103

120.K40 Revise W-211 FDC for AN-107

120.L37 Provide Documentation for W.TBO Validation

120.L40 Prep W-TBO Pij Definition/Toch Basis/FDC

120.L41 241-AN-102 Upgrades: Prop Tech Rqmnits \& CDR

120.M40 241-AN-106 Upgrades: Prop Tech Ramnls \& CDR

120.P32 Validate SY.102 Design to DST Specilication

120.R40 Reviso W-TBD Design Specilications for SY-101

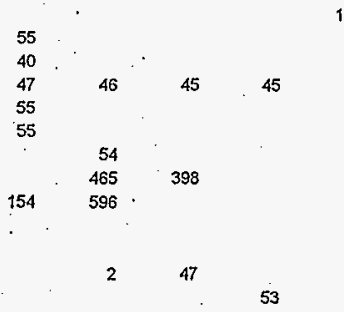

103 
Table 4. Cost Loading Report

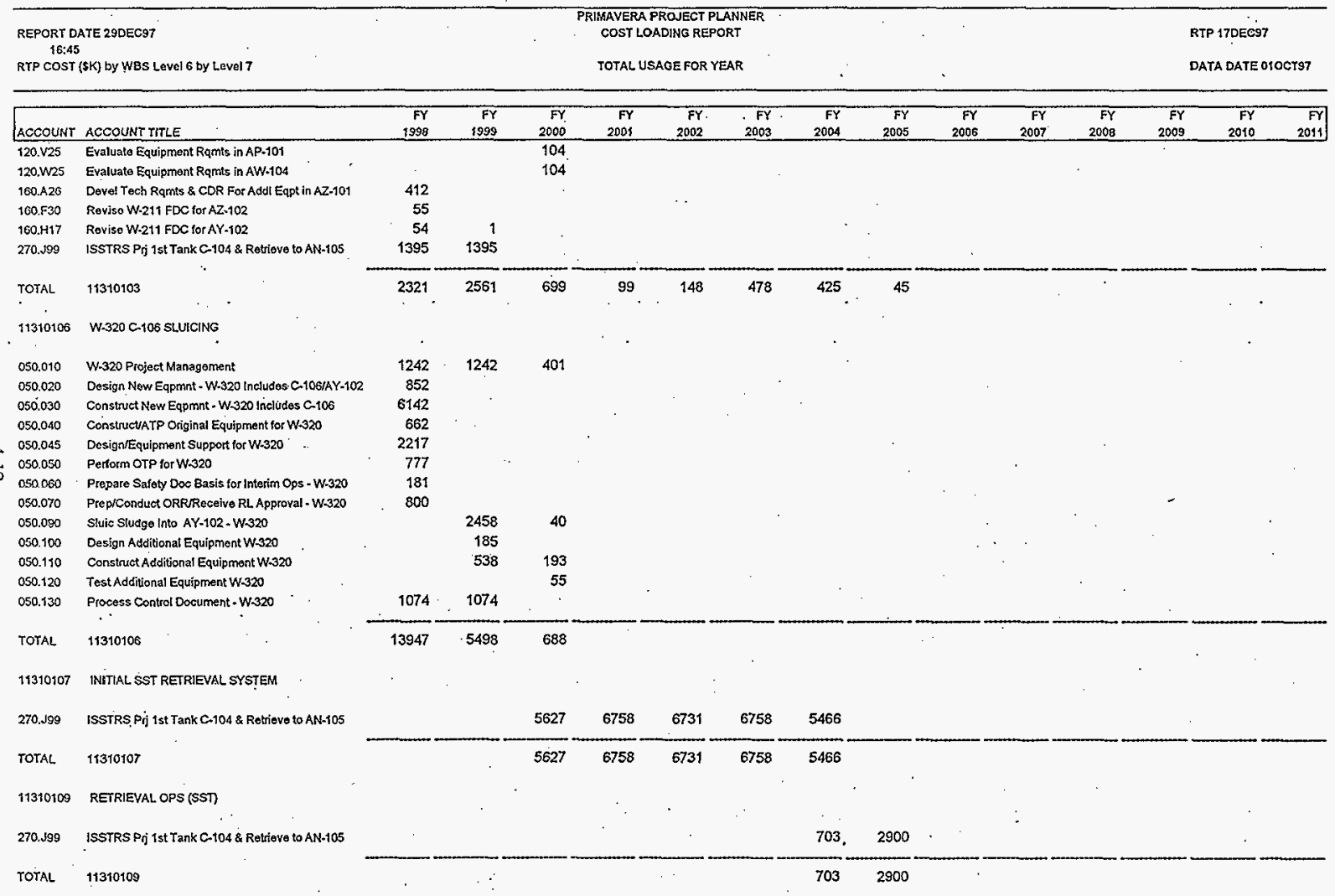


: Table 4. Cost Loading Report

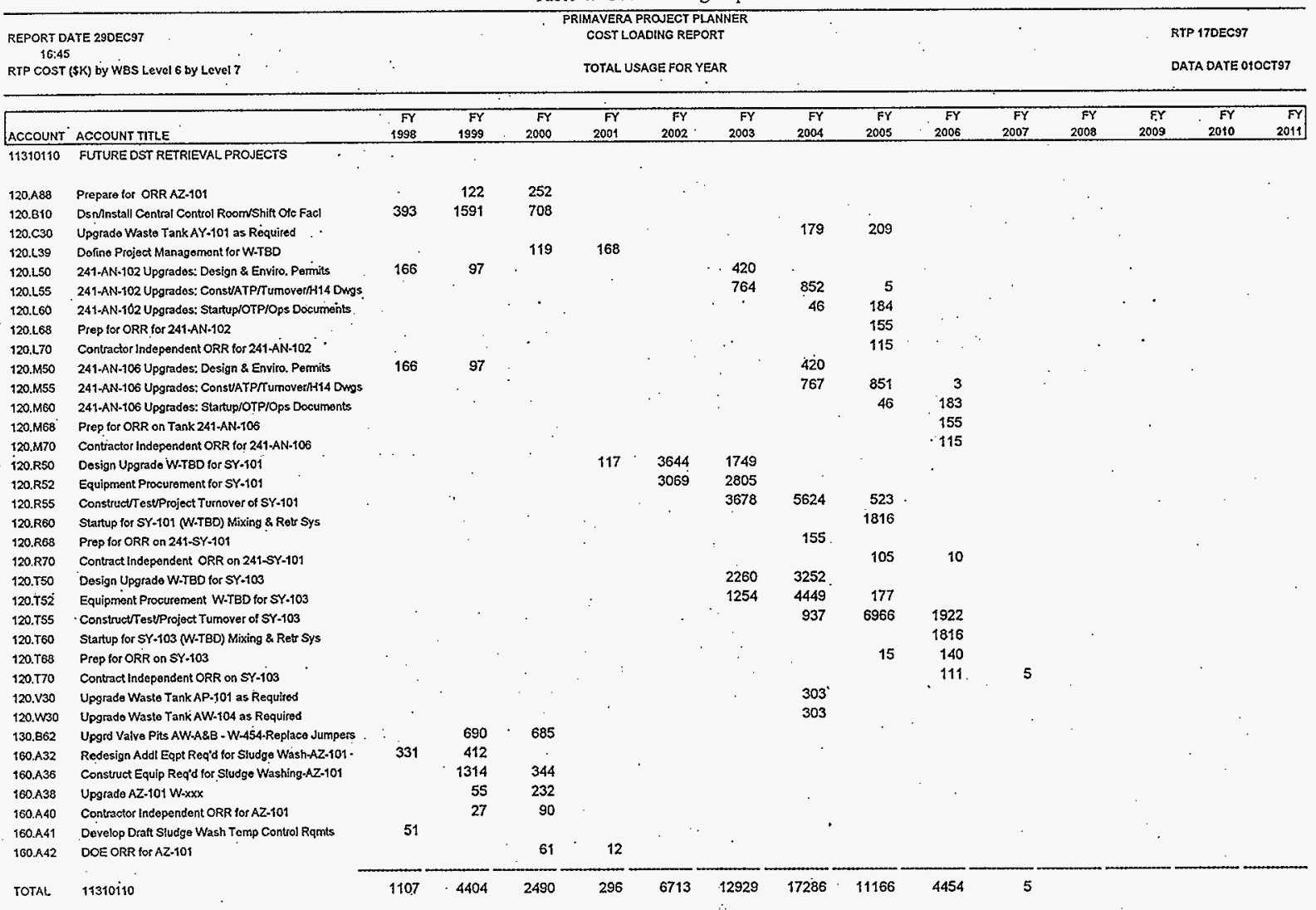


Table 4. Cost Loading Report

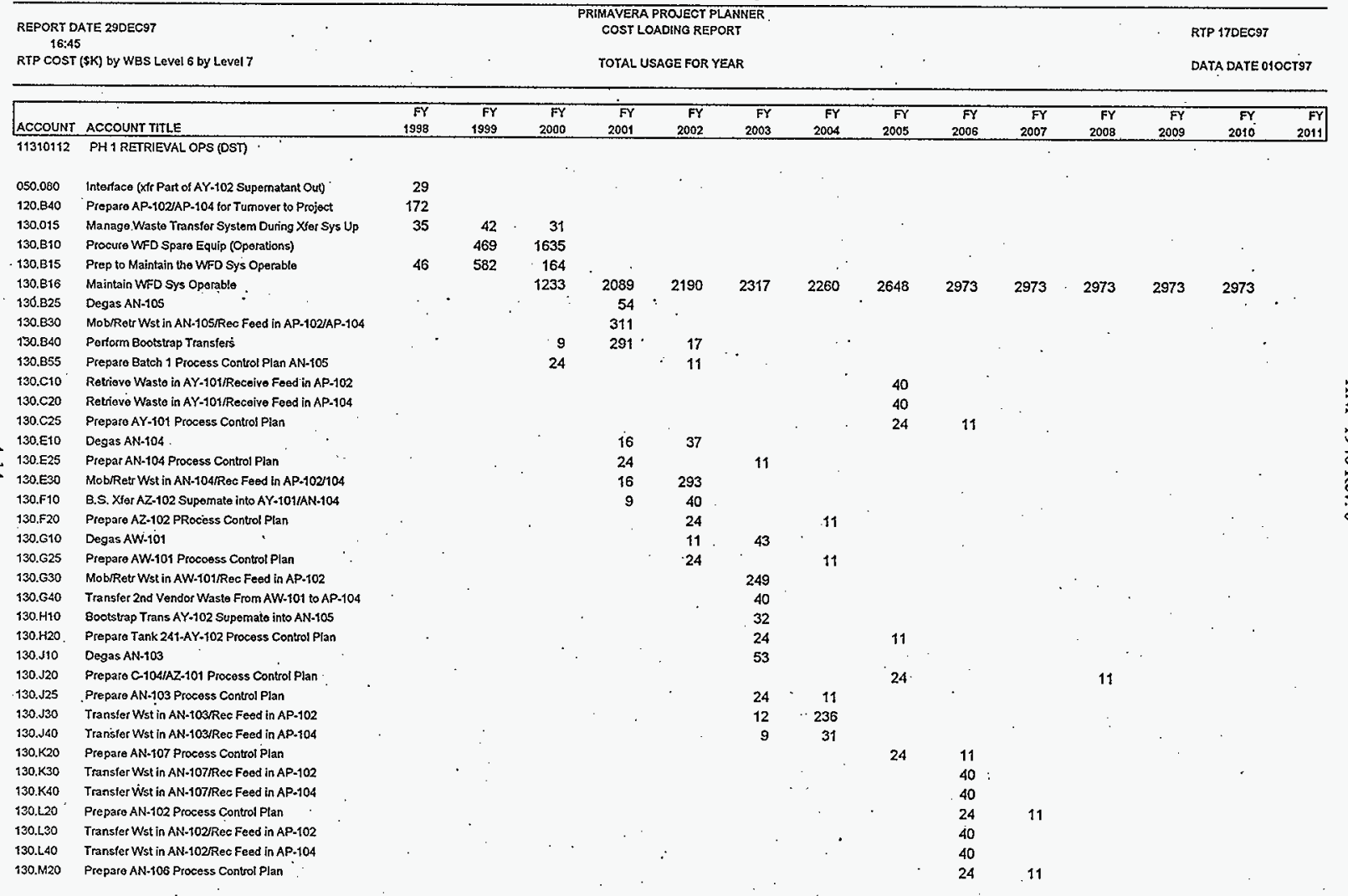


Table 4. Cost Loading Report

PRIMAVERA PROJECT PLANNER

COST LOADING REPORT

RTP 17DEC97

$16: 45$

RTP COST (\$K) by WBS Level 6 by Level 7

TOTAL USAGE FOR YEAR

DATA DATE $010 \mathrm{OCT} 97$

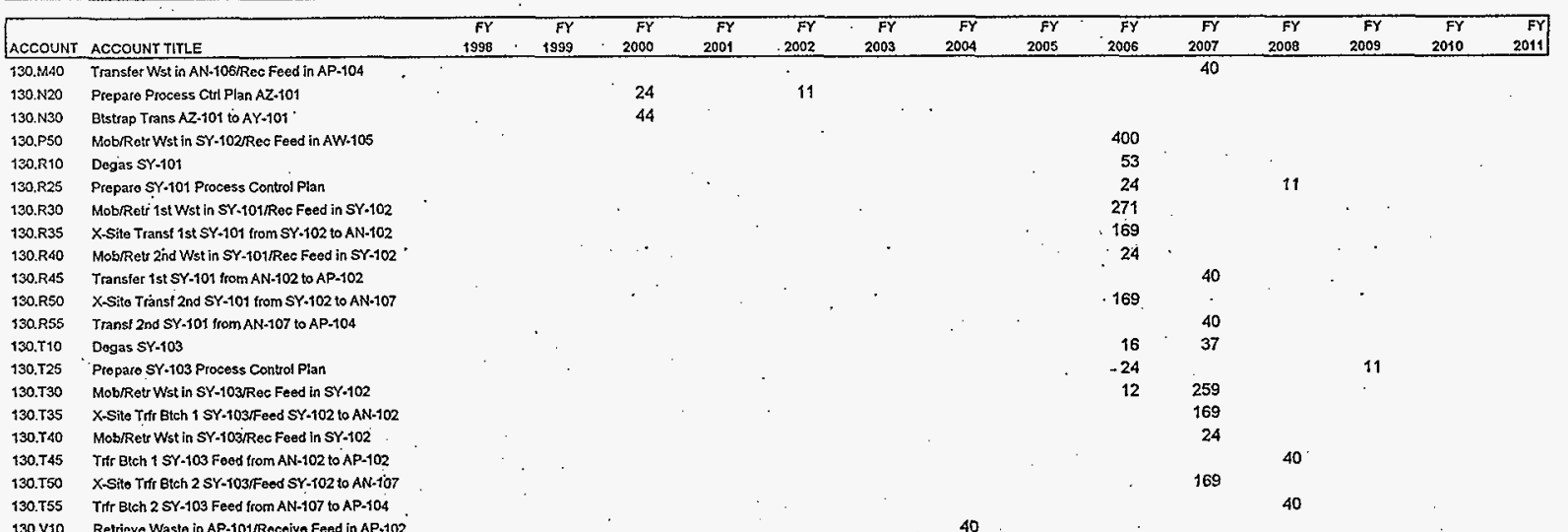

$130 . \mathrm{V10}$ Retro

130.V20. Retrievo Waste in AP-101/Receive Feed in AP. 104

11

130.W10 Retrieve Wasto in AW-104Recolvo Foed in AP-102

24

130.W20 Retreye Waste in AW-104/Receive Feed in AP-104

150.B32 Mix AN-10́5 Feed in AP+102

150.B42 Adjust AN-105 Feed in AP-102

150.B44 Deliver AN-105 Feed to AP-106

150.B48 Mix AN.105 Feed in AP.104

150.B58 Adjust AN-105 Feed in AP-104

150.860 Deliver AN-105 Feed to AP-108

150.CO5 Mix AY-101 Feed in AP.102

150, C 30 Adjust AY-101 Feed in AP-102

150,C35 DeliverAY-101 Feed from AP-102 to AP-106

150.C40 Mix AY-101 Feed in AP.104

150.C65 Adjust AY-101 Feed in AP-104

$\begin{array}{rr}4 & 26 \\ 161 & 9 \\ . & 35 \\ 4 & 26 \\ 110 & 59 \\ & 35\end{array}$

Adjust AY-101 Feed in AP-104 
Table 4. Cost Loading Report

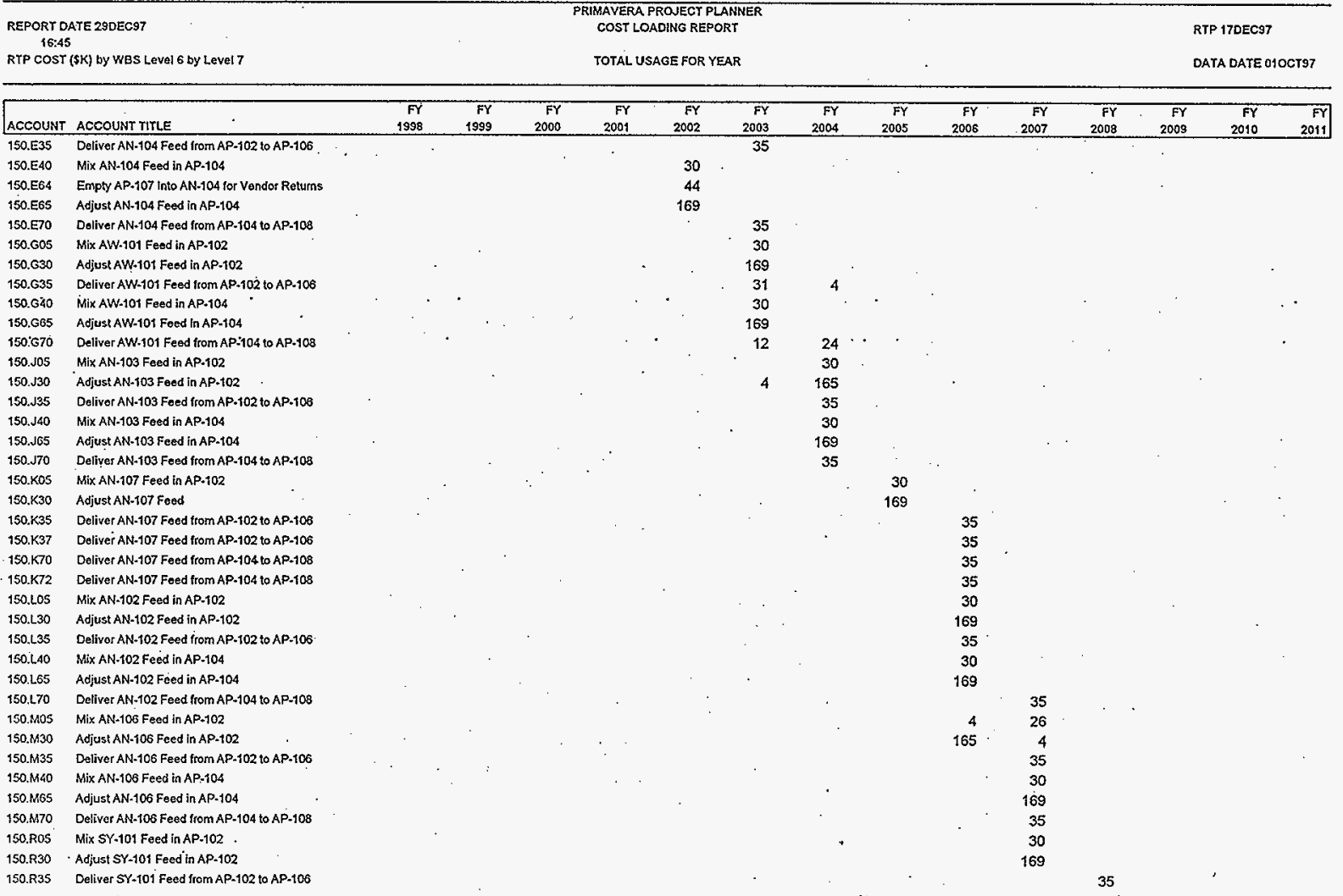


Table 4. Cost Loading Report

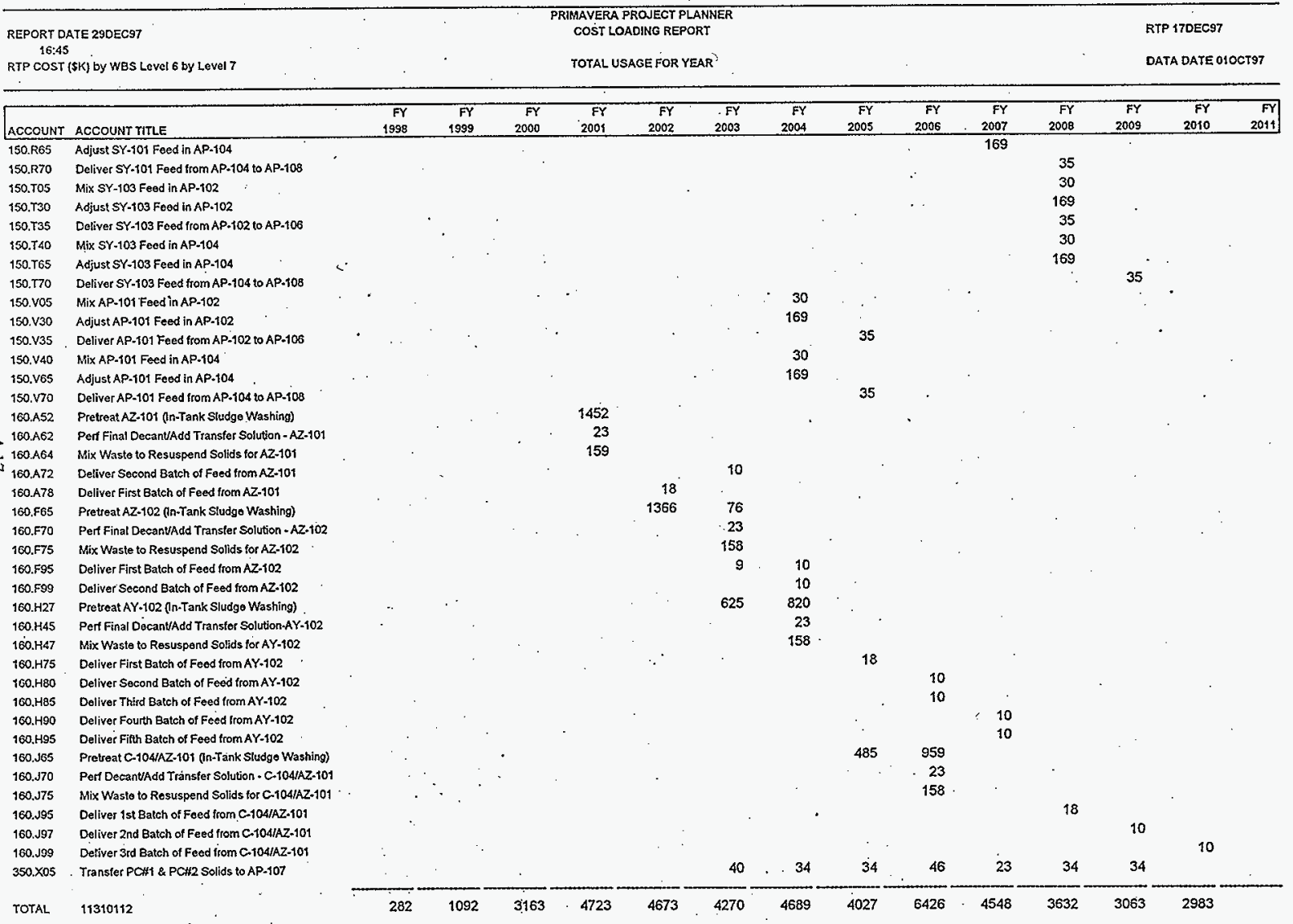


Table 4. Cost Loading Report

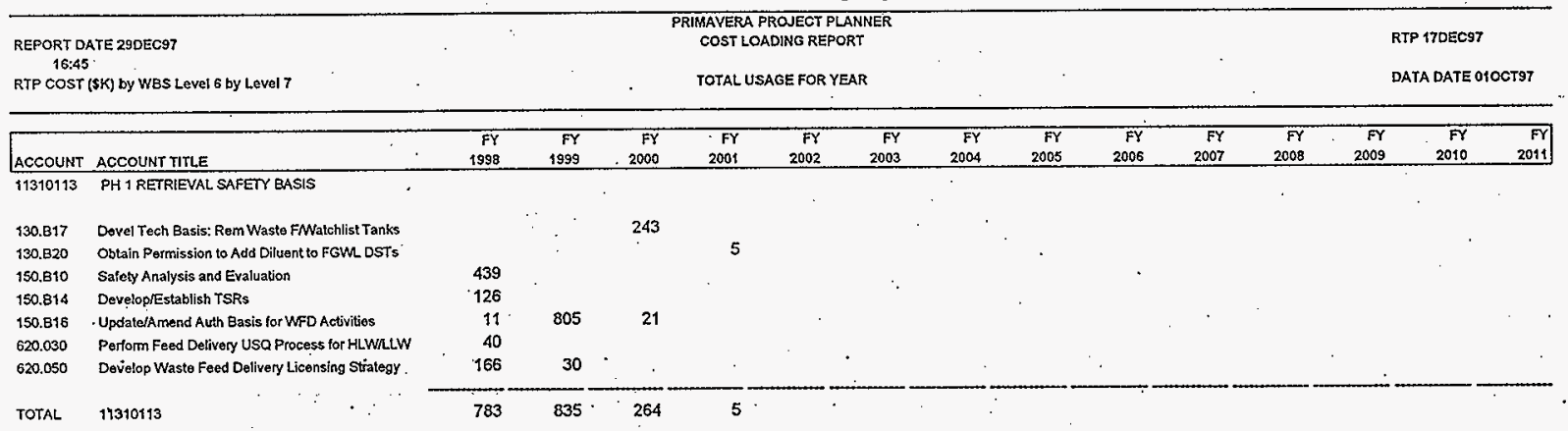

11310114 PH 1 RETR OPS (CHARACTERIZATION)

150.B34 Obtain AN-105 Feed Qual Samples from AP-102

150.B36 Provide AN-105 Samples from AP-102 to PC 1

150.838 Analy AN-105 Smpisinterprot Results from AP-102

150.640 Prov AN-105 Feed Batch Info from AP-102 to PC 1

150.B50 Obtain AN-105 Focd Qual Samples from AP-104

150.852 Provide AN-105 Samples from AP-104 to PC 2

150.854 Analy AN-105 Smpls/nterpret Results from AP-104

150.856 Provide AN-105 Feed Batch Info to PC2

150.c10 Obtain AY-101 Feed Qual Samples from AP-102

150.C15 Provido AY-101 Samples from AP-102 to PC 1

150.C20 Analy AY-101 Smpls/nterpret Resutts from AP.102

150.C25 Provide AY-101 Feed Batch Into to PC 1

150.C45 Obtain AY-101 Food Qual Samples from AP-104

$150 . C 50$ Provide AY.101 Samples from AP-104 to PC2

150.C55 Analy AY+101 Smpls/interpret Results from AP-104

150.C60 Prov AY-10s Feed Batch Into from AP-104 to PC2

150.E10 Obtain AN-104 Feed Qual Samplos from AP-102

150.E15 Provide AN-104 Samples from AP-102 to PC1

150.E20 Analy AN-104 SmplsAnterpret Results from AP-102

150.E25 Provide AN-104 Feed Batch Info to PC 1

150.E45 Obtain AN-104 Feed Qual Samples from AP-104

150.E50 Provido AN-104 Samples from AP. 104 to PC 2

150.E55 Analy AN-104 Smpls/nterpret Results from AP-104

150.E60 Prov AN-104 Feed Batch info from AP-104 to PC2

73

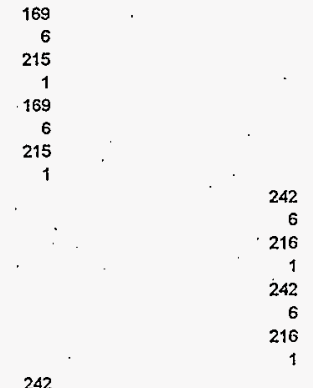


Table 4. Cost Loading Report

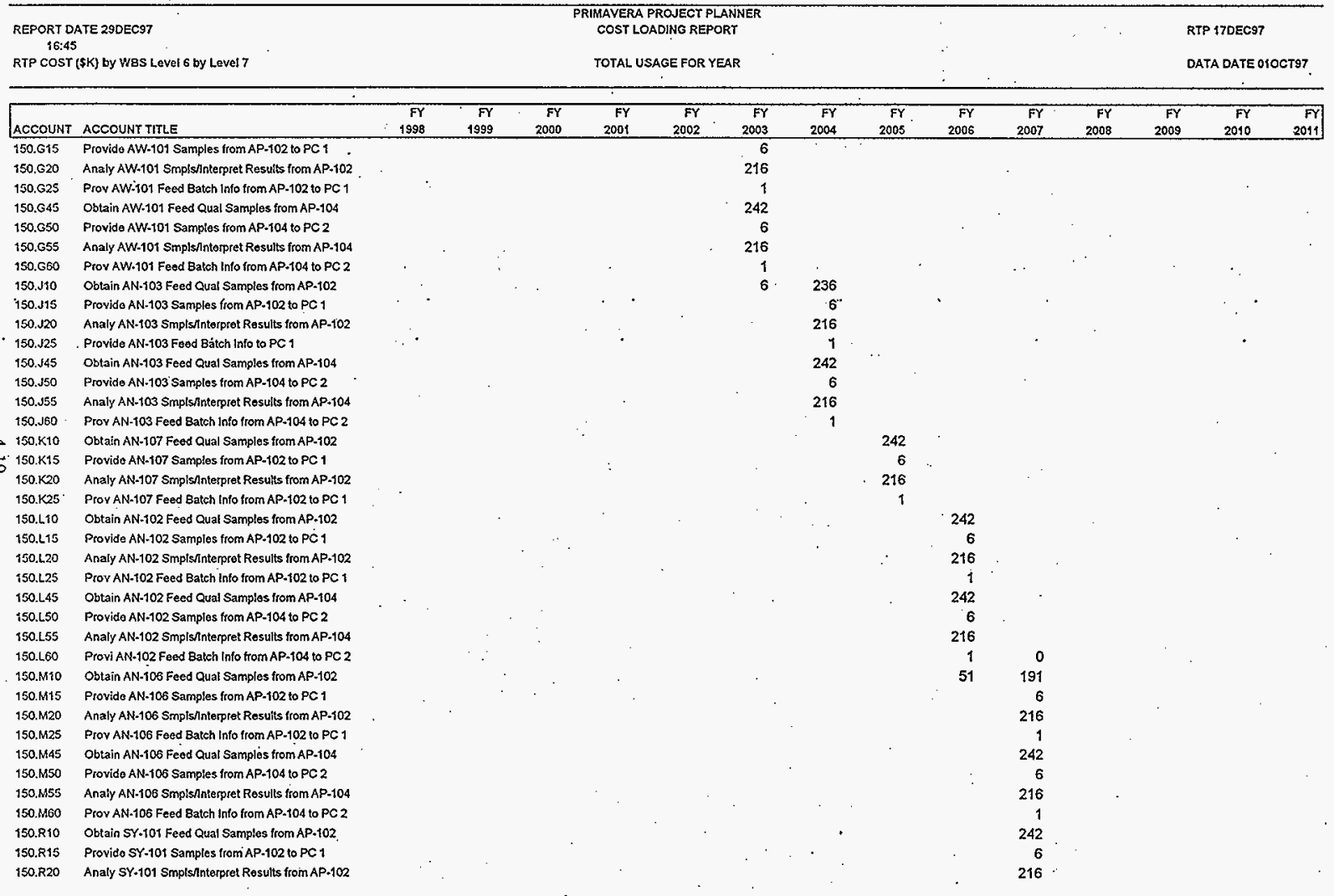


Table 4. Cost Loading Report

PRIMAVERA PROJECTPLANNER

COST LOADING REP,ORT

RTP $170 E C 97$

$16: 45$

TOTAL USAGE FOR YEAR

DATA DATE $010 \mathrm{CTS7}$

RTP COST (\$K) by WBS Level 6 by Level 7

\begin{tabular}{|c|c|c|c|c|c|c|c|c|c|c|c|c|c|c|c|}
\hline ACCOUNT & ACCOUNT TITLE & $\begin{array}{r}F Y \\
1998 \\
\end{array}$ & $\begin{array}{r}F Y \\
1999 \\
\end{array}$ & $\begin{array}{r}F Y \\
2000 \\
\end{array}$ & $\begin{array}{r}F Y \\
2001 \\
\end{array}$ & $\begin{array}{r}F Y \\
2002 \\
\end{array}$ & $\begin{array}{r}F Y \\
2003 \\
\end{array}$ & $\begin{array}{r}F Y \\
2004 \\
\end{array}$ & $\begin{array}{r}F Y \\
2005 \\
\end{array}$ & $\begin{array}{r}F Y \\
2006 \\
\end{array}$ & $\begin{array}{r}F Y \\
2007 \\
\end{array}$ & $\begin{array}{r}F Y \\
2008 \\
\end{array}$ & $\begin{array}{r}F Y \\
2009 \\
\end{array}$ & $\begin{array}{r}F Y \\
2010 \\
\end{array}$ & $\begin{array}{r}F Y \\
2019\end{array}$ \\
\hline 150.R45 & Obtain SY-101 Feed Qual Samples from AR-104 & & & & & & & +3 & & & 242 & & & & \\
\hline 150.R50 & Provido SY-101 Samples from AP-104 to PC 2 & & & & & & & & & & 6 & & & & \\
\hline 950.855 & Analy SY-101 Smpls/ntorprat Results from AP-104 & & & & & & & & & & 207 & 9 & & & \\
\hline $150 . \mathrm{R} 60$ & Prov SY-101 Feod Batch Info from AP-104 to PC 2 & & & - & & & & & & & & 1 & ${ }^{\circ}$ & & \\
\hline 150.T10 & Obtain SY-103 Feod Qual Samplos from AP-102 & & & & & & & & & & • & 242 & & & \\
\hline $150 . T 15$ & Provido SY.103 Samples from AP-102 to.PC 1 & & & . & & & & & & & & 6 & & & \\
\hline 150,720 & Analy SY.103 Smplssinterpret Results from AP. 102 & & & & & & & & & & & 216 & & . & \\
\hline $150 . T 25$ & Prov SY-103 Feed Bateh info from AP-102 to PC 1 & & & & & & & & & & & 1 & & & \\
\hline $150 . T 45$ & Obtain SY-103 Feed Qual Samples from AP-104 & & & $\cdot$ & & & ' & & & & & 242 & $\cdot$ & & \\
\hline 150.150 & Provide SY-103 Samples from AP: 104 to PC 2 & & & & & & & & & & & 6 & & & \\
\hline 150.755 & Analy SY-i03 Smp/sinterpret Results from AP-104 & & & • & $=$ & & $\because$ & & & & & 216 & $\cdot$ & & \\
\hline $150 . \mathrm{T} 60$ & Prov SY-103 Feed Batch Info from AP-104 to PC 2 & & & & & & & & & & & 1 & & & \\
\hline
\end{tabular}

- Prov SY-103 Feed Batch Info from AP-104 to PC 2

150.V15 Provis-101 Feed Qual Samples from AP.102

150.V20 Analy AP.101 Smplsinterpret Results from AP.102

if 150.v25 Provide AP-101 Feed Batch Into to PC1

150.V45 Obtain AP-101 Feed Qual Samples from AP-104

i50.v50 Provide AP-101 Samples from Ap-104 to PC 2

i50.v55 Analy AP-101 Smplsinterpret Results from AP-104

150.V60 Prov AP-101 Foed Batch Into From Ap: 104 to PC2

160-A25 Update B31 \& TCR to Reflect Procoss Test AZ-101

160.A66 Obtain HiLWFeed Qual Grab Samples $A Z$-101 solids

160.A68 Eval Samples/Establish MT for AZ-101

160 A74 Provide Separate Samples to the PC -AZ-101

160.A76 Prov MT Non-Volatile Oxides Exclude NaISi-AZ-101

160.F50 Obtain HLW Feed Qual Grab Sämplos AZ-102 Solids

160.F55 Eval Smpls:Estab MTof Non-Volatile for AZ-102

160.F85 Provide Separate Samples to Private Contractor

160.890 Provide MT Non-Volatilo Oxides Excluding Na/Si

160.H25 Confirm that AY-102 is Acceptable for Feed to PC

160.H50 Obtain HLW Feed Qual Grab Samples AY-102 Solids

160. H55 Eval Smpls/Estab MT of Non-Volatilo for AY-102

160,465 Frovide MT Non-Volatile Oxides Excluding Na/Si

160.H70 Provide Separate Samples to PC.AY-102

160.J50 Obtain HLWFeed Qual Grab Samp C-104/AZ-101

160.J55 Eval Smpls/Estab MT of Non-Volatile for C-104

160.J85 Provido Separate Samples to Privato Contractor

160. 390 Provide MT Non-Volatilo Oxides Excluding Na/Si

TOTAL - 11310114 
Table 4. Cost Loading Report

\begin{tabular}{|c|c|c|}
\hline $\begin{array}{l}\text { REPORT DATE 29DEC97 } \\
\quad \text { 16:45 }\end{array}$ & $\begin{array}{l}\text { PRIMAVERA PROJECT PLANNER } \\
\text { COST LOADINO REPORT } \\
\text {. }\end{array}$ & RTP $17 D E C 97$ \\
\hline RTP COST (SK) by WBS Level 6 by Level 7 & . TOTAL USAGE FOR YEAR & DATA DATE $010 \mathrm{CT} 97$ \\
\hline
\end{tabular}

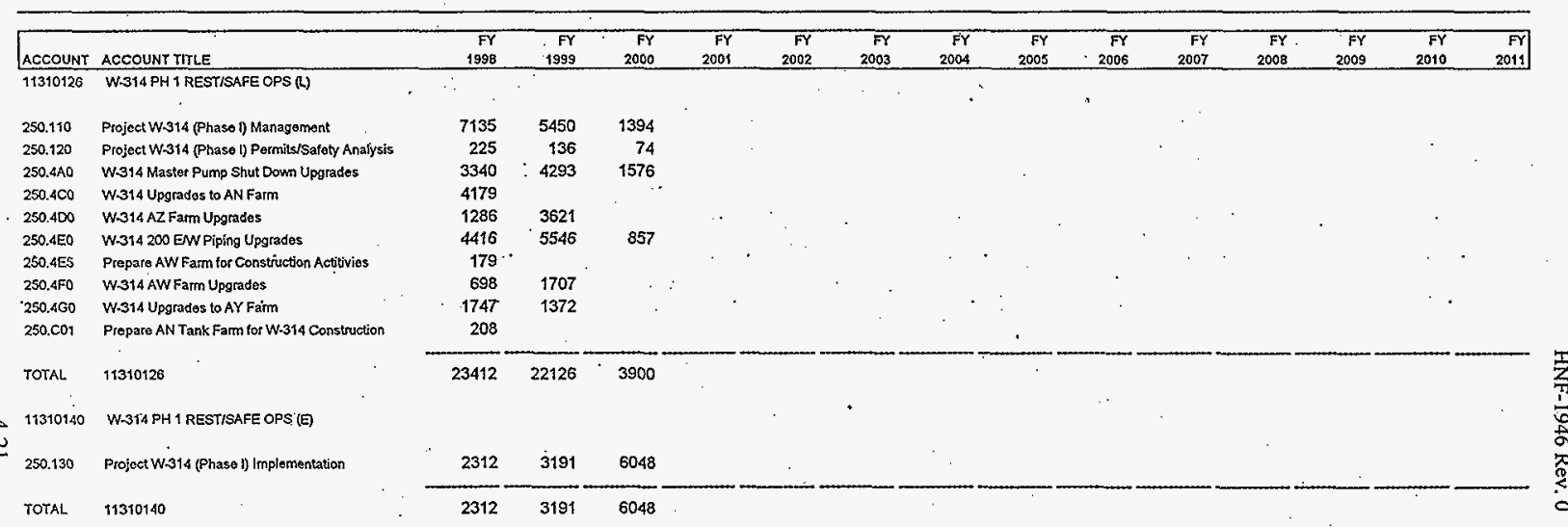

1131014E PROJECT-W.151 101.AZ RETRIEVAL (L)

\subsection{W-151 Project Managernent \\ 110.050 Conduct LMHC Readiness Assessment \\ 110.070 Pertorm Mixer Pump Test $(W-151)$}

TOTAL 1131014E

1131014P PROJECT W-15t 101-AZ PROCESS TEST

110.010 W-151 Projert Management .

110.045 Revise/ss Mixer Pump Test Plans/Proceduses

110.070 Perform Mixer Pump Test (W-151)

110.090 Prepare Test Report (W-i51)

TOTAL $1131014 \mathrm{P}$

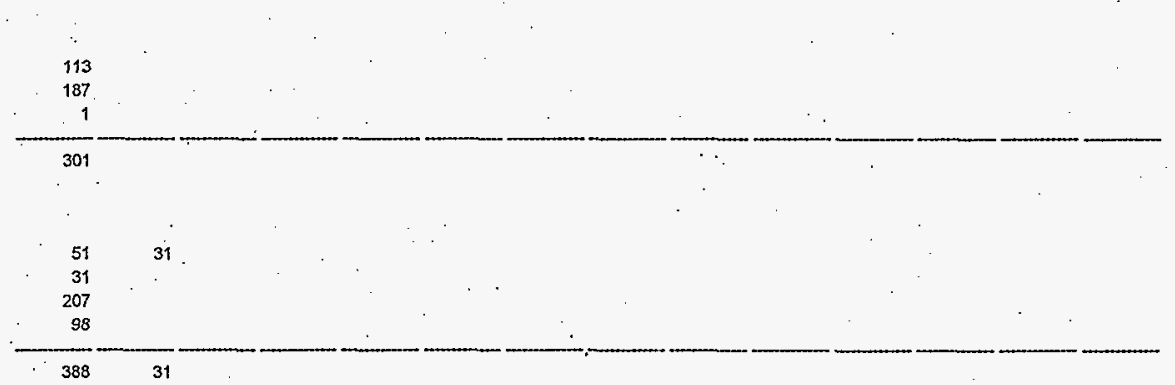


Table 4. Cost Loading Report

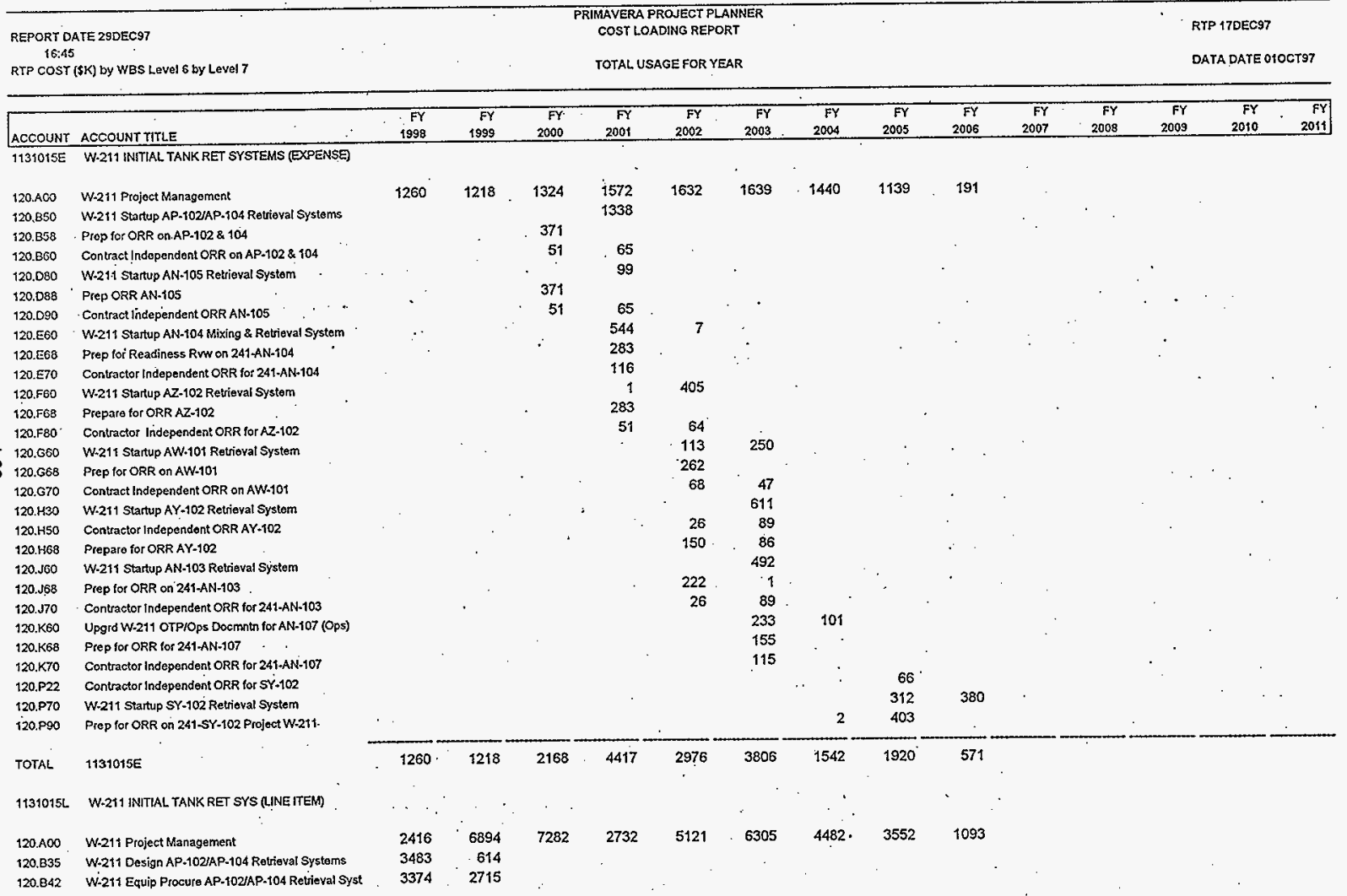


Table 4. Cost Loading Report

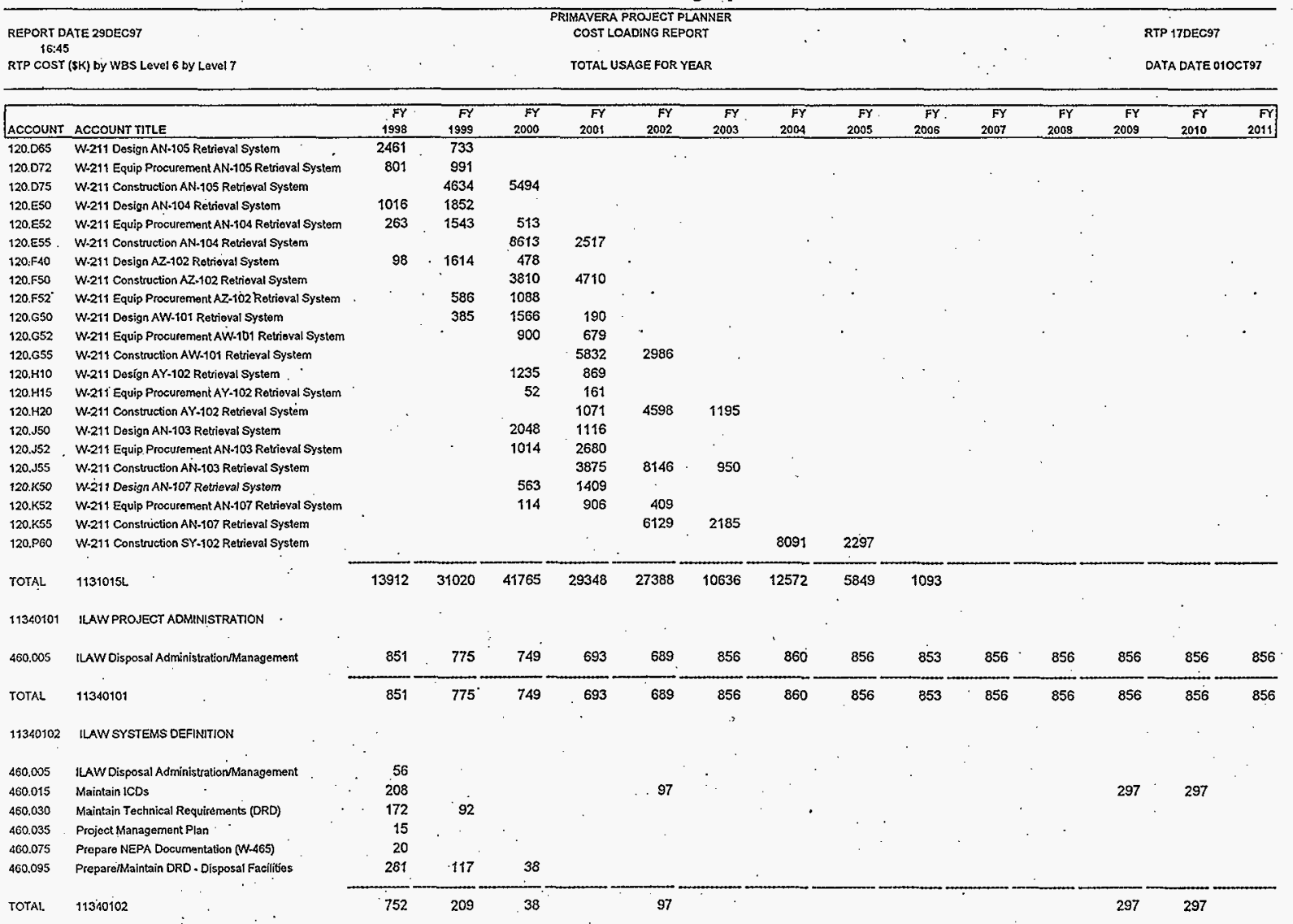


Table 4. Cost Loading Report

PRIMAVERA PROIECT PLANNER

COST LOADING REPORT

RTP 17DEC97

REPORT OATE 29DEC9T

TOTAL USAGE FOR YEAR

DATA DATE $010 C T 97$

RTP COST (\$K) by WBS Level 6 by Level 7

\begin{tabular}{|c|c|c|c|c|c|c|c|c|c|c|c|c|c|c|}
\hline ACCOUNT ACCOUNT TIILE் & $\begin{array}{r}F Y \\
1998\end{array}$ & $\begin{array}{r}F Y \\
1999\end{array}$ & $\begin{array}{r}F Y \\
2000\end{array}$ & $\begin{array}{r}F Y \\
2001\end{array}$ & $\begin{array}{r}\mathbf{F Y} \\
2002\end{array}$ & $\begin{array}{r}F Y \\
2003\end{array}$ & $\begin{array}{r}F Y \\
2004\end{array}$ & $\begin{array}{r}F Y \\
2005\end{array}$ & $\begin{array}{r}F Y \\
2006\end{array}$ & $\begin{array}{r}F Y \\
2007\end{array}$ & $\begin{array}{r}F Y \\
2008\end{array}$ & $\begin{array}{r}F Y \\
2009\end{array}$ & $\begin{array}{r}F Y \\
2010\end{array}$ & $\begin{array}{r}F Y \\
2011\end{array}$ \\
\hline
\end{tabular}

11340104 PRONECT W-465

440.110 Prepare NEPA Documentation (W-464)

440.120 Authorization 8asis Development \& ApDr (W-464)

460.015 Maintain ICDs

460.040 (LAW Storage Conceptual Dosign (W-465)

460.045 ILAW Storage Adv Conceptual Design (W-465)

460.060 Project Validation (W-465)

460.065 LLAW Storage Detailed Dosign (W-465)

460.070 Madify Vaults (W-465)

460.075 Prepare NEPA Dociumentation (W-465)

460.080 Preparo RCRA Pormits (W-465)

460.085 Authorization Basis Development \& A App (W.4\$5)

470.010 Procedures $/$ raining/OTPJORR (nitial Phase l)

点

TOTAL 11340104

$\$ 1340105$ ILAWOPERATIONS

460.160 Data Collection for Performance Assessment

460.170 Maintaia ILAW Phase I Performance Assessmont

$470.010^{\circ}$ Procedurestraining/OTPIORR (nnitial Phase I)

470.040 interim Store ILAW Operations (Initial Phase l)

470.060 Procedutes/TrainingiOTPJORR (Balanco Phasa l)

470.075 Dispose lLAW Operations (Batance Phase 1)

TOTAL 11340105

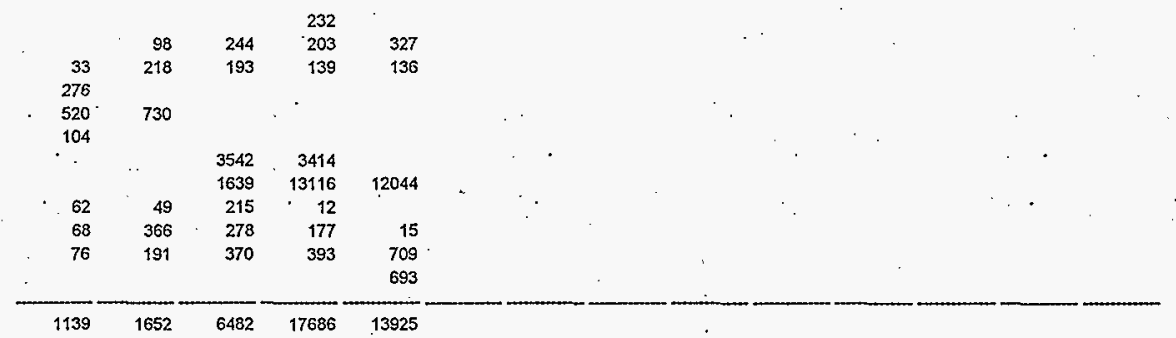

11340106 PROJECT W.520 FUTURE FACILITIES

460.105 Conceptual Deslan - Disposal Facilities (W-520)

460.150 Advanced Conceptual Dsn - Disposal Fac (W-520)

460.112 Validate Project $(W-520)$

460.115 Design Disposal Facilities (W-520)'

460.120 Construct Disposal Facilities (W.520)

460.125 Regulatory Requirements for Disposal Facilities

460.130 Authorization Basis Development and Approval

TOTAL 11340106

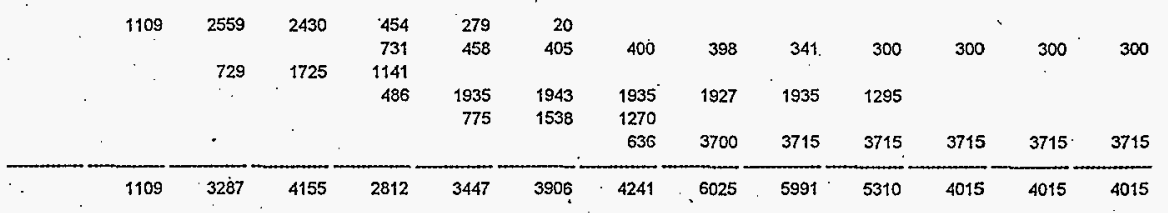

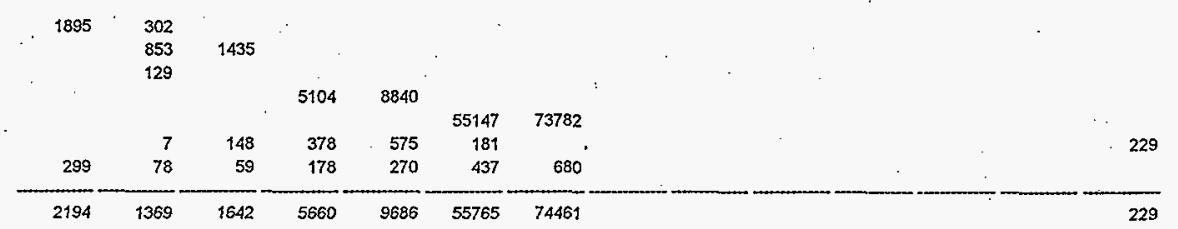


Table 4. Cost Loading Report

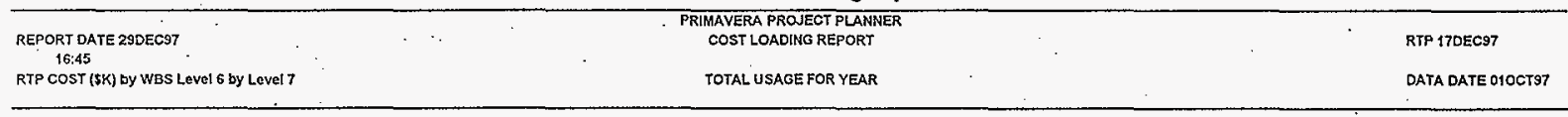

\begin{tabular}{|c|c|c|c|c|c|c|c|c|c|c|c|c|c|c|c|}
\hline ACCOUNT & ACCOUNT TITLE & $\begin{array}{r}F Y \\
1998 \\
\end{array}$ & $\begin{array}{r}F Y \\
1999 \\
\end{array}$ & $\begin{array}{r}F Y \\
2000 \\
\end{array}$ & $\begin{array}{r}F Y \\
2001 \\
\end{array}$ & $\begin{array}{r}F Y \\
.2002 \\
\end{array}$ & $\begin{array}{r}F Y \\
2003 \\
\end{array}$ & $\begin{array}{r}F Y \\
2004 \\
\end{array}$ & $\begin{array}{r}F Y \\
2005 \\
\end{array}$ & $\begin{array}{r}F Y \\
2006 \\
\end{array}$ & $\begin{array}{r}F Y \\
2007 \\
\end{array}$ & $\begin{array}{r}\overline{F Y} \\
2008 \\
\end{array}$ & $\begin{array}{r}F Y \\
2009 \\
\end{array}$ & $\begin{array}{r}F \gamma \\
2010\end{array}$ & $\begin{array}{r}F Y \\
2011 \\
\end{array}$ \\
\hline 11340107 & ILAWFACILTYY CLOSURE AND D\&D & & & & & & & & & & . & & & & \\
\hline 470.080 & Close ILAW Disposal Facilities & & & & & & & & & & & 602 & 1513 & 902 & 902 \\
\hline TOTAL & 11340107 & & & & & & . & & & & & 602 & 1513 & 902 & 902 \\
\hline $1134013 B$ & Obtain Borehole \#1 Samples (C) & & : & & & & & & $\cdot$ & & & & & & . \\
\hline 460.145 & Data Coilection for 2001 Performance Asséssment. & 132 & & & & & & . & & & & & & & . \\
\hline TOTAL & $1134013 B^{\circ}$ & 132 & . & & & & & & & & & & & & \\
\hline $1134013 E$ & ILAW PERFORMANCE ASSESSMENT (日) & & & & & & & & & & & & & & \\
\hline 460.140 & 1998 Performance Assesssment & 443 & 11 & & & . & & & & & & & & & \\
\hline 460.145 & Data Collection for 2001 Performance Assossment & 3087 & 3465 & 944 & 291 & & & & & & & & & & \\
\hline 460.155 & 2001 Performance Assessment & & & 326 & 114 & 5 & & & & & . & & & & \\
\hline TOTAL & $1134013 E$ & 3531 & 3476 & 1270 & 405 & 5 & & & & & & & & & \\
\hline
\end{tabular}

1134013M X.Ray Microtomography Equip (C)

460.145 Data Collection for 2001 Performance Assessment

TOTAL 1134013M

\begin{tabular}{|c|c|c|c|c|c|c|c|c|c|c|c|c|c|}
\hline 120 & & & & & & & & & & & & & \\
\hline & . & & & & & & & . & & & & & \\
\hline 170 & 107 & 104 & 138 & 138 & 138 & 139 & 138 & 138 & 138 & 138 & 138 & 138 & 138 \\
\hline 170 & 107 & 104 & 138 & 138 & 138 & 139 & 138 & 138 & 138 & 138 & 138 & 138 & 138 \\
\hline
\end{tabular}

TOTAL 11340201

11340202 - HHLW SYSTEMS DEFINITION

440.010 IHLW Interim Storege Administration/Managemont
440.020 Prepare/Maintain ICDS (DD 13,14,17)
440.030 Project Management Plans
440.040 Maintain Technical Reçuirements (DRD)
490.090 Negetiate RW Requiremsnts

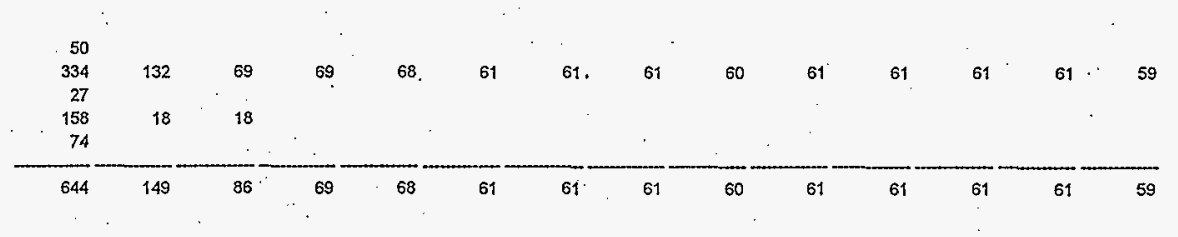


Table 4. Cost Loading Report

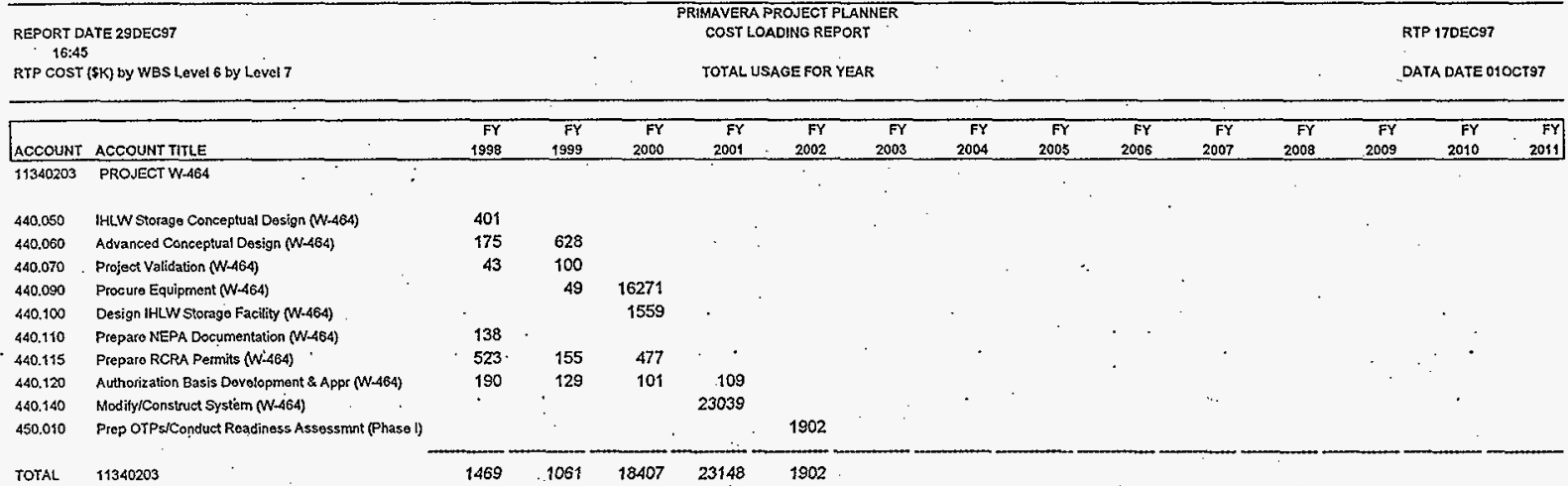

f 11340204 IHLW OPERATIONS

is

450,030 TransportRoceive/nterim Storo LHLW (Phase 1)

TOTAL 11340204

11380101 PH-1 INFR. PROJ. MGT/ADM.

330.010. Project Administration/Management/Engineating.

TOTAL $\quad 11380101$

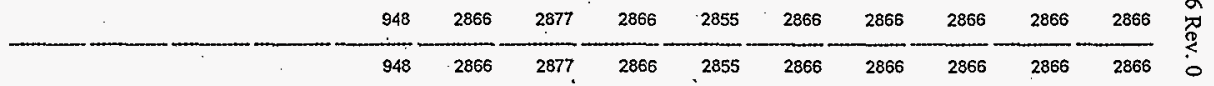

11390102 PH-1 INFR. SYSTEM DEFINITION

150.866 Ready PHMC Operthaint Doc-AP-108 Tumover 150.B76 Ready PHMC OperMaint Doc-AP-106 Turnover

150,888 Prep AP-106 for Tumover to Private Contractor

150.894 Estabilish Interface Regmnts tor Tank Tumover

150.B98 Prep AP-108 for Turnover to Privato Contractor

330.020 Systems Definition

TOTAL 11380102

\begin{tabular}{|c|c|c|c|c|c|c|c|c|c|c|c|c|c|}
\hline 358 & 464 & 378 & 419 & 212 & 213 & 214 & 213 & 212 & 213 & .213 & 213 & 213 & 141 \\
\hline 358 & 464 & 378 & 419 & 212 & 213 & 214 & 213 & 212 & 213 & 213 & 213 & 213 & 141 \\
\hline
\end{tabular}

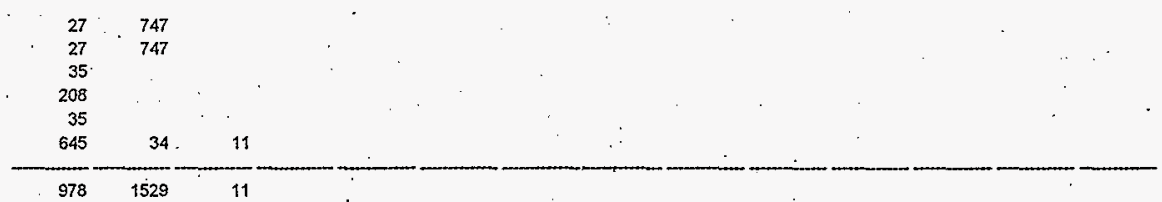


Table 4. Cost Loading Report

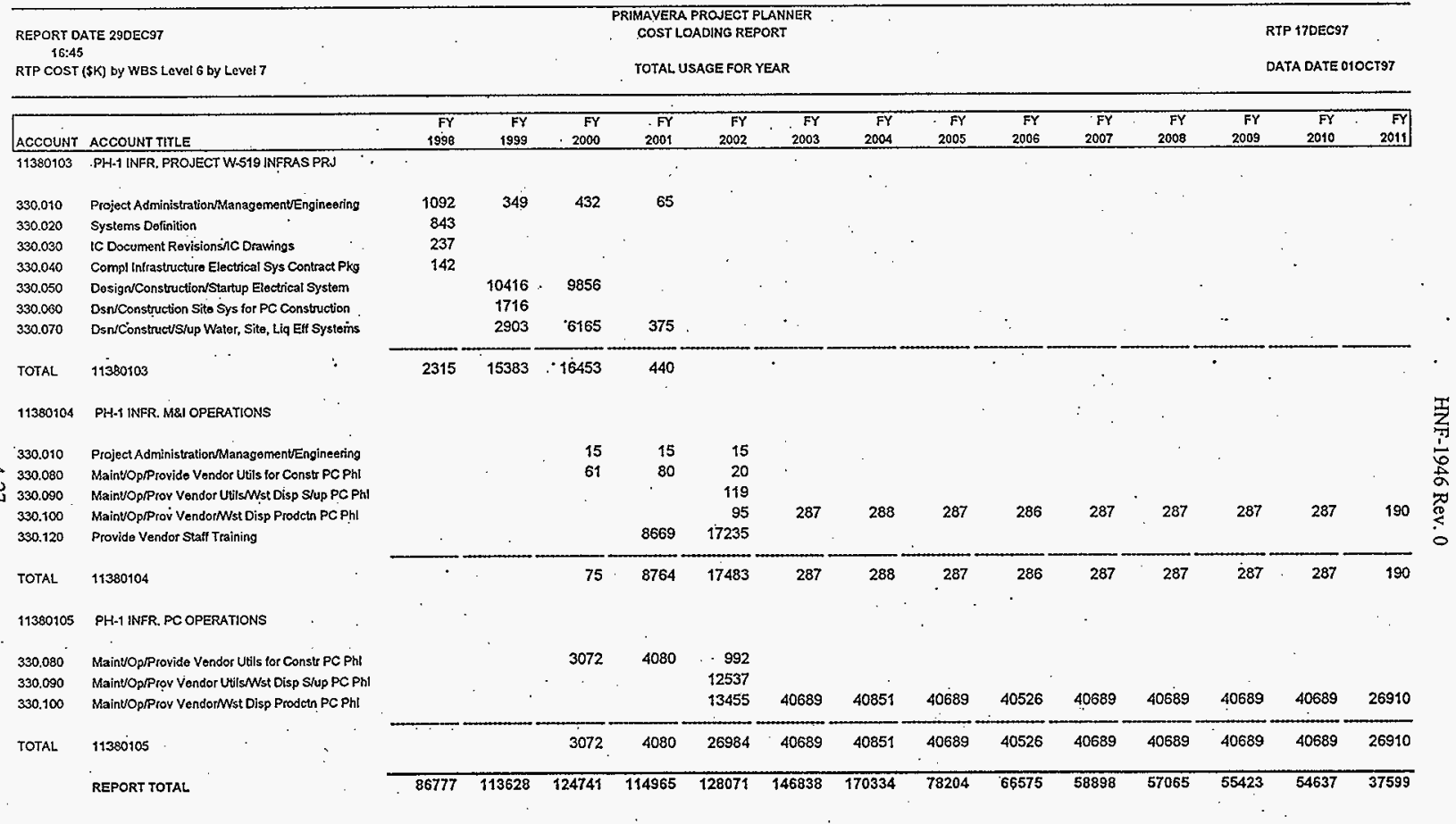




\subsection{SIGNIFICANT PROGRAM-LEVEL CHANGES (SCOPE, SCHEDULE, COST)}

This section is a program summary-level discussion of the scope, schedule, and cost changes between the TWRS FY 1998 MYWP (Lenseigne 1997) and the revised RTP planning included in the Initial Updated Baseline. This discussion addresses the RTP scope and costs shown in Table 3, Summary Cost Spreadsheet, for "Phase 1B Logics." Additional TWRS Project costs related to Phase 1 (for scope not in the Logics) have been included in this Initial Updated Baseline. For a detailed discussion of these other costs, refer to the Financial Analysis (Wells 1998).

- The Initial Updated Baseline represents a more thorough analysis of the scope, schedule and cost than is contained in the FY 1998 MYWP. The RTP planning has benefitted from the systematic and controlled RTP planning process previously described in Section 1.0. This process has ensured that the higher level mission direction and requirements defined for the TWRS Project have been properly allocated through the technical logics and WBS to the detailed planning level (executable tasks) for each of the program elements. The RTP planning reflects development of mature Level 1 Logics where previously only preliminary Mid-Level Logics existed for the MYWP. The RTP planning also reflects improved scope and interface definition resulting from the use of the multi-functional, integrated planning teams and preparation of TBR packages. The basis of estimates are well documented in the TBR packages.

This section is provided to highlight the major changes related to scope, schedule and costs. Detailed comparisons to the MYWP are of limited value due to the significant RTP advances in the logic and detailed planning basis. The RTP. Initial Updated Baseline will serve as the basis for a change request to revise the MYWP Baseline.

\subsection{RETRIEVAL PROJECT}

The Retrieval Project has been significantly replanned during RTP for Waste Feed Delivery activities shown on the Level 1 Logics and documented in the TBR packages. Activities for Feed Process System Definition and Retrieval Operations have received enhanced scope definition and planning detail from that shown in the TWRS FY 1998 MYWP (Lenseigne 1997). Extensive changes in these areas resulted in the revised Project Master Baseline Schedule shown on Figure 8 in Section 3.3. Major changes are as follows:

- Addition of two (2) feed batches to provide adequate LAW Envelope " $\mathrm{A}$ " and HLW Envelope " $D$ " feed to the private contractors. The Envelope " $A$ " feed batch will be provided from tanks AP-101 and AW-104 and the Envelope "D" batch will be provided from single-shell tank (SST) C-104 (first tank from the Initial Single-Shell Tank Retrieval System)

- Planning definition and detail related to sampling and characterization for feed definition, process control, and feed qualification 
- $\quad$ Planning definition and detail related to Double-Shell Tank (DST) Operations to deliver Phase $1 \mathrm{LAW}$ and HLW feed batches and receive a solid slurry return waste stream back from the private contractors

- Rescoping of Project W-TBD to add two (2) mixer pump retrieval systems in tanks SY-101 and SY-103. The AN tanks previously planned will be retrieved with transfer pumps only

- Validation of the planning for the feed delivery Safety Authorization Basis amendment and addition of resources for RL Tier II and III reviews

- Completion of Project W-454 upgrades

- Numerous activities related to systems definition, design development, and testing for SSTs, DSTs, mixer pumps and sludge washing.

Schedule changes reflect significant replanning to address the added and deleted work scope as well as the revised logic to support the private contractors' feed delivery schedule. In general, schedule changes reflect:

- The addition of the new feed batches within the Phase 1 time constraints

- Acceleration of critical activities, as required, to reduce schedule risk and provide slack

- $\quad$ Data Quality Objectives work

- $\quad$ Project W-211 tank specific Functional Design Criteria

- . Project definition and upgrades work for DST retrieval systems beyond Project W-211.

- Design concept work for the Initial SST Retrieval System.

The RTP estimates for Phase 1 reflect an $\$ 84$ Million increase from the MYWP. This increase is attributable to many factors in the revised planning (additions, deletions, and revised schedule). However, the following are two primary contributors:

- Increase in the Phase 1 DST Retrieval Operations of $\$ 40.0$ Million. A majority of these replanned operations ( $\$ 47.0$ Million) were not costed in the MYWP.

- Approximately $\$ 46.0$ Million for the additional LAW and HLW feed batches including tank upgrades and operations. 
HNF-1946 Rev. 0

\subsection{IMMOBILIZED WASTE}

Based on preparation of Level 1 Logics and associated baseline review and updates the following changes are noted:

- $\quad$ Replanned or added activities to address:

- $\quad$ Safety Authorization Bases and Environmental Impact Statement Supplemental Analysis (C-2 Analysis)

- Project Management Plans .

- Internal interface control documents

- $\quad$ Compliance with proposed DOE Order 435.1

- $\quad$ Preparation of Level 1 specifications for projects

- Updated planning (engineering studies) for Advanced Conceptual Design

- Revisions to correct cost entry errors in the MYWP

- Revision to reflect the Project W-520, Module 1 Facility Decontamination and Decommissioning/Closure as a Phase 2 cost.

The schedule changes reflect work added, deleted, or moved consistent with the Level 1 Logics and TBR packages. Significant changes are:

- Acceleration of existing grout vaults closure from FY.2011 to FY 2009

- Waste delisting petition and rulemaking petiton to U.S. Environmental Protection Agency shifted out 1 year (previous change requests)

- $\quad$ Additional activities to reflect ILAW project critical path strategy through completion of Operational Readiness Review and Critical Decision 4 by RL.

Overall, the RTP costs for Immobilized Waste is $\$ 14.7$ Million lower than the MYWP. However, $\$ 11.9$ Million is due to the movement of the W-520 Module 1 Decontamination and Decommissioning /Closure activities to Phase 2. The balance of the cost delta is due to correction of MYWP entry errors and Safety Authorization Basis replanning. 
HNF-1946 Rev. 0

\subsection{PRIVATIZATION INFRASTRUCTURE (Phase 1)}

Based on preparation of Level 1 Logics and associated baseline review and updates, the following changes are noted.

- $\quad$ Extended Program/Project Management and Administration from FY 2001 through FY 2011 (not in MYWP).

- $\quad$ Added and deleted numerous Systems Definition activities (net cost decrease).

- Moved Phase 1 Management and Integration Operations support to the deactivation of the private contractors' facilities from Phase 1 to Phase 2 (beyond June 2011).

- Added costs for training of the private contractors' operators (FY 2001 and. FY 2002) and utilities to support operation of the private contractors' facilities (FY 2003 and FY 2004); these were not funded in the FY 1998 MYWP (below the line in the MYWP).

Schedule changes reflect work added, deleted, or moved consistent with the Level 1 Logics and TBR packages. Significant changes are:

- Extension of Program/Project Management and Administration from FY 2001 through FY 2011

- Acceleration of waste management definition and feed tank turnover supporting activities to FY 1998

- Completed operations support (utilities) to the private contractors in June 2011 consistent with their plant operations. Utilities to support private contractors' deactivation are a Phase 2 cost.

Overall, the $\mathrm{RTP}$ costs for Privatization Infrastructure are $\$ 105.7$ Million higher than the FY 1998 MYWP. This delta is attributable to scope not funded (below the line) in the MYWP for the training of private contractors' operators in FY 2001 and FY.2002 and utility costs to support operation of the private contractors' facilities in FY 2003 and FY 2004.

Please note that the RTP estimate for Phase 1 private contractors' operations includes $\$ 155.3$ Million for liquid effluent processing. Based on a determination that this scope of work is fully funded as part of the Waste Management Program, these costs are not required. A line item adjustment in Table 3 under "Retrieval Scope Transfer Shortfalls" is shown to delete the costs. 


\subsection{REFERENCES}

\section{Department of Energy Orders}

DOE Order 435.1 (proposed), Radioactive Waste Management, U.S. Department of Energy, Washington, D.C.

\section{Documents}

Acree, C. D., Jr., 1998, Tank Waste Remediation System Mission Analysis Report, HNF-SD-WM-MAR-008, Rev. 2, DRAFT, prepared by Lockheed Martin Hanford Corporation for Fluor Daniel Hanford, Inc., Richland, Washington.

Boston, H. L., and K. N. Jordan, 1998, Tank Waste Remediation System Retrieval and Disposal Mission Readiness-to-Proceed Memorandum, HNF-2019, Rev. 0, DRAFT, prepared by Lockheed Martin Hanford Corporation for Fluor Daniel Hanford, Inc., Richland, Washington.

DNFSB, 1993, DNFSB Recommendation 93-5 to the Secretary of Energy Regarding Hanford Tank Sampling and Characterization, DNFSB 93-5, Defense Nuclear Facility Safety Board., Washington, D.C.

Ecology, EPA, and DOE, 1996, Hanford Federal Facility Agreement and Consent Order, 2 vols., Washington State Department of Ecology, U.S. Environmental Protection Agency, and U.S. Department of Energy, Olympia, Washington.

Freeman, D. V., 1998, Tank Waste Remediation System Program Plan, HNF-1883, Rev. 0, DRAFT, prepared by Lockheed Martin Hanford Corporation for Fluor Daniel Hanford, Inc., Richland, Washington.

Kirkbride, R. A., G. K. Allen, P. J. Certa, A. F. Manuel, R. M. Orme, L. W. Shelton, E. J. Slaathaug, R. S. Wittman, and G. T. MacLean, and D. L. Penwell (SESC), 1997, Tank Waste Remediation System Operation and Utilization Plan, HNF-SD-WM-SP-012, Rev. 0, Volumes I and II, prepared by Numatec Hanford Corporation for Fluor Daniel Hanford, Inc., Richland, Washington.

Lenseigne, D. L., 1997, Tank Waste Remediation System Fiscal Year 1998 Multi-Year Work Plan WBS 1.1, HNF-SP-1230, Rev. 0, prepared by Lockheed Martin Hanford Corporation for Fluor Daniel Hanford, Inc., Richland, Washington. 
Payne, M. A., P. J. Certa, J. O. Honeyman, J. G, Kristofzski, and W. T. Thompson, 1998, Management Assessment of Tank Waste Remediation System Contractor Readiness to Proceed With Phase 1B Privatization, HNF-2021, Rev. 0, DRAFT, prepared by Lockheed Martin Hanford Corporation for Fluor Daniel Hanford, Inc., Richland, Washington.

Peck, L. G., 1998, Tank Waste Remediation System Systems Engineering Management Plan, HNF-SD-WM-SEMP-002, Rev. 1, DRAFT, prepared by Lockheed Martin Hanford Corporation for Fluor Daniel Hanford, Inc.; Richland, Washington.

Treat, R., P. Bartley, T. J. McLaughlin, R. D. Potter, R. E. Raymond, and W. L. Willis; 1998, Tank Waste Remediation System Retrieval and Disposal Mission Technical Baseline Summary Description, HNF-1901, Rev. 0, DRAFT, prepared by Lockheed Martin . Hanford Corporation for Fluor Daniel Hanford, Inc., Richland, Washington.

Wells, M. W., 1998, Tank Waste Remediation System Retrieval and Disposal Mission Phase 1 Financial Analysis, HNF-2017, Rev. 0, DRAFT, prepared by Lockheed Martin Hanford Corporation for Fluor Daniel Hanford, Inc., Richland, Washington. 
HNF-1946 Rev. 0

APPENDIX A

LEVEL 1 LOGICS 
HNF-1946 Rev. 0

This page intentionally left blank.

A-ii 


\section{CONTENTS}

\section{LOW-ACTIVITY WASTE FEED LEVEL 1 LOGICS}

Figure A-1 LAW 1st Feed Batches, Tank 241-AN-105

Figure A-2 LAW 2nd Feed Batches, Tank 241-AN-104

Figure A-3 LAW 3rd Feed Batches, Tank 241-AW-101 2

Figure A-4 LAW 4th Feed Batches, Tank 241-AN-103 ' 2

Figure A-5 LAW 5th Feed Batches, Tanks 241-AP-101 \& 241-AW-104 : 2

Figure A-6 LAW 6th Feed Batches, Tank 241-AY-101 2

Figure A-7 LAW 7th \& 8th Feed Batches, Tank 241-AN-107 2

Figure A-8 LAW 9th Feed Batches, Tank 241-AN-102 2

Figure A-9 LAW 10th Feed Batches, Tank 241-AN-106 ? 2

Figure A-10 LAW 11th Feed Batches, Tank 241-SY-101 2

Figure A-11 LAW 12th Feed Batches; Tank 241-SY-103

\section{HIGH-LEVEL WASTE FEED LEVEL 1 LOGICS}

Figure A-12 HLW 1st \& 2nd Feed Batches, First Tank 241-AZ-101 3

Figure A-13 HLW 3rd \& 4th Feed Batches, First Tank 241-AZ-102 3

Figure A-14 HLW 5th - 9th Feed Batches, 241-AY-102 3

Figure A-15 HLW 10th - 12th Feed Batches, Tank 241-C-104 3

\section{IMOBILIZED LOW-ACTIVITY WASTE LEVEL 1 LOGICS}

Figure A-16 Immobilized Waste ILAW

IMMOBILIZED HIGH-LEVEL WASTE LEVEL 1 LOGICS

Figure A-17 Immobilized Waste IHLW

\section{INFRASTRUCTURE PHASE 1 LEVEL 1 LOGICS}

Figure A-18 Phase 1 Privatization Support 
HNF-1946 Rev. 0

This page intentionally left blank. 
INF-1946 Rev. 0

APPENDIX B

\section{GUIDANCE AND REQUIREMENTS TO DELIVERABLES CROSSWALK}

Tank Waste Remediation System Retrieval and Disposal Initial Updated Baseline Summary 
HNF-1946 Rev. 0

This page intentionally left blank.

B-ii 
Table B-1. Tank Waste Remediation System Retrieval and Disposal Mission Initial Updated Baseline Summary.

\begin{tabular}{|c|c|c|}
\hline Guidance or Requirement & Status & Implementing Location \\
\hline $\begin{array}{l}\text { A } 1 \text { DOE Letter to H Hatch, FOH from W J } \\
\text { Taylo, DOE, dated August } 8,1997,49757162 \mathrm{~A}(36 \\
\text { [TEM CHECKLIST) }\end{array}$ & \% & (2) \\
\hline 3. Requirements are allocated to functions. & I & HNF 1946 , Sections 2.1 and 2.4 \\
\hline $\begin{array}{l}\text { 4. Each activity is tied to and necessary to support a } \\
\text { contractual requirement. }\end{array}$ & I & Complete Document \\
\hline $\begin{array}{l}\text { 7. Technical logic are complete at all levels; the } \\
\text { program makes sense logically; and lower level-logic } \\
\text { rolls up properly to higher-level logic. }\end{array}$ & I & $\begin{array}{l}\text { Sections } 1.2,2.1,2.2,2.4,2.5 \text {, } \\
\text { and } 3.3\end{array}$ \\
\hline $\begin{array}{l}\text { 10. Interface control documents have been prepared, } \\
\text { DOE comments have been resolved, issues have } \\
\text { been addressed, and approved interfaces are } \\
\text { managed. }\end{array}$ & I & Sections $1.2,2.4,2.5$, and 3.3 \\
\hline $\begin{array}{l}\text { 11. Waste feed plans and implementing actions and } \\
\text { procedures are on track. }\end{array}$ & I & Sections $1.2,2.4,2.5$, and 3.3 \\
\hline $\begin{array}{l}\text { 12. Infrastructure support plan and implementing } \\
\text { actions and procedures are on track. }\end{array}$ & I & Sections $1.2,2.4,2.5$, and 3.3 \\
\hline $\begin{array}{l}\text { 13. Immobilized waste product project plans and } \\
\text { implementing actions and procedures are on track. }\end{array}$ & I & Sections $1.2,2.4,2.5$, and 3.3 \\
\hline $\begin{array}{l}\text { 18. System reliability, availability, and } \\
\text { maintainability have been assessed and are sufficient } \\
\text { to support processing rate requirements. }\end{array}$ & I & Sections $1.2,2.4,2.5,3.3$ \\
\hline $\begin{array}{l}\text { 19. The physical integrity of existing systems, } \\
\text { structures, and components has been verified. }\end{array}$ & I & Sections $1.2,2.4,2.5,3.3$ \\
\hline $\begin{array}{l}\text { 20. Waste has been characterized as necessary to } \\
\text { satisfy all data quality objectives. }\end{array}$ & 1 & Sections $1.2,2.4,2.5,3.3$ \\
\hline $\begin{array}{l}\text { 22. Resource-loaded estimates have been completed } \\
\text { for all activities. }\end{array}$ & I & Sections 1.2, 3.3, 4.1, 4.2, 4.3 \\
\hline $\begin{array}{l}\text { 23. The critical path is identified and float has been } \\
\text { calculated. }\end{array}$ & I & Section $1.2,3.2$ and 3.3 \\
\hline 24. Activities have been properly funded. & I & Section 4.1 \\
\hline $\begin{array}{l}\text { 26. There is agreement among scope, budget, and } \\
\text { schedule. The scope of work can be performed } \\
\text { within budget and on schedule. }\end{array}$ & I & Sections $1.1,1.2,2.4,3.3$ and 4.3 \\
\hline $\begin{array}{l}\text { 32. Environmental permits and safety authorization } \\
\text { bases are in place as required for authorization-to- } \\
\text { proceed decision. }\end{array}$ & I & Sections $1.2,2.4,2.5,3.3$ \\
\hline
\end{tabular}


Table B-1. Tank Waste Remediation System Retrieval and Disposal Mission Initial Updated Baseline Summary.

\begin{tabular}{|c|c|c|}
\hline Guidance or Requirement & Status & Implementing Location \\
\hline $\begin{array}{l}\text { A. } 2 \text { DOE Letter to H J Hatch, FDH, from W/J } \\
\text { Taylor, DOE, dated August 8, 1997, \#97571621 } \\
\text { (BODY OF TEXT) }\end{array}$ & का & \\
\hline \multicolumn{3}{|l|}{$\begin{array}{l}\text { General PHMC Responsibilities from RL's 8/8/97 } \\
\text { letter, Section } 2.1\end{array}$} \\
\hline $\begin{array}{l}\text { 2. Develop a resource-loaded, logic driven schedule, } \\
\text { including critical path }\end{array}$ & I & Sections $1.2,2.4$, and 3.3 \\
\hline $\begin{array}{l}\text { 5. Costs and schedules in the programmatic baseline } \\
\text { can be met }\end{array}$ & I & Sections 1.1, 1.2, 2.4, 3.3, 4.3 \\
\hline $\begin{array}{l}\text { 6. Critical path analysis is done to determine the } \\
\text { impacts of budget shortfalls on the programmatic } \\
\text { baseline }\end{array}$ & I & Sections 3.2 and 3.3 \\
\hline $\begin{array}{l}\text { A.3 DOE Letter H.J Hatch, FDH, from William } \\
\text { J. Taylor, o oE, dated December } 2,1997 \text {, } \\
\text { \#9761291 }\end{array}$ & 1.\%. & \\
\hline $\begin{array}{l}\text { 6. RTP deliverables and their support must address } \\
\text { the readiness of supporting facilities outside of } \\
\text { TWRS, and understand FDH's readiness to receive } \\
\text { secondary wastes. }\end{array}$ & I & Section 2.6 \\
\hline $\begin{array}{l}\text { 7. Establishment of sound technical baseline and } \\
\text { update M\&I TWRS Retrieval Baseline. Submit } \\
\text { Level } 1 \text { logic diagrams and documentation, an } \\
\text { identified critical path, and a listing of documents } \\
\text { that form the basis for the technical baseline for } \\
\text { January RTP. }\end{array}$ & I & Sections $1.1,1.2,2.4$ and 3.3 \\
\hline \multicolumn{3}{|l|}{$\begin{array}{l}\text { 11. Make a QA plan available for DOE review in } \\
\text { January. }\end{array}$} \\
\hline $\begin{array}{l}\text { b. Address corresponding resources (for the QA } \\
\text { implementing procedures) }\end{array}$ & I & Sections 1.2 and 3.0 \\
\hline \multicolumn{3}{|l|}{$\begin{array}{l}\text { B.1 poE Order } 430.1 \text {, Good Practice Guider } \\
\text { GPG-NM-002 }\end{array}$} \\
\hline \multicolumn{3}{|l|}{ 2.2.4 Baseline: Cost Criteria } \\
\hline 1. Total Estimated Cost (TEC) & 1 & Sections $1.2,4.1,4.2$, and 4.3 \\
\hline 2. Life Cycle Cost (LCC) & I & Sections 1.2 and 4.0 \\
\hline \multicolumn{3}{|l|}{ 2.2.5 Baseline: Schedule Criteria } \\
\hline 1. Milestones & $\mathrm{I}$ & Sections $3.2,3.3$ and 3.4 \\
\hline 2.3.2 Funding Profile & I & Section 4.0 \\
\hline
\end{tabular}


Table B-1. Tank Waste Remediation System Retrieval and Disposal Mission Initial Updated Baseline Summary.

\begin{tabular}{|c|c|c|}
\hline Guidance or Requirement & Status & Implementing Location \\
\hline 2.3.4 Baseline: Cost Criteria & $\therefore$ & \\
\hline 1. Total Estimated Cost (TEC) & $\mathrm{I}$ & Section $1.2,4.1,4.2,4.3$ \\
\hline 2. Total Project Cost (TPC) & 1 & Sections $1.2,4.1,4.2$, and 4.3 \\
\hline 3. Life Cycle Cost (LCC) & $I$ & Sections 1.2 and 4.0 \\
\hline 2.3.5 Baseline: Schedule Criteria & . & 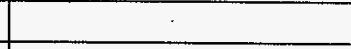 \\
\hline 1. Milestones & I & Sections $3.2,3.3$, and 3.4 \\
\hline 2.4.2 Funding Profile & $\mathrm{I}$ & Sections 4.0 \\
\hline 2.4.4 Baseline: Cost Criteria & . & \\
\hline 1. Total Estimated Cost (TEC) & $\mathrm{I}$ & Sections $1.2,4.1,4.2$ and 4.3 \\
\hline 2. Total Project Cost (TPC) & 1 & Sections $1.2,4.1,4.2$, and 4.3 \\
\hline 3. Life Cycle Cost (LCC) & $\mathrm{I}$ & Sections 1.2 and 4.0 \\
\hline 2.4.5 Baseline: Schedule Criteria & . & \\
\hline 1. Milestones & I & Sections $3.2,3.3$, and 3.4 \\
\hline 2. Master Schedule & $\mathrm{I}$ & Section 1.2 and 3.3 \\
\hline 3. Thresholds & I & $\begin{array}{l}\text { Refer to HNF-1900 (CM) and } \\
\text { WHC-IP-0842, Vol. VIII, } \\
\text { Section 1.1, Rev. } 0 \text { (Change } \\
\text { Control). }\end{array}$ \\
\hline 2.5.4 Baseline: Cost Criteria & $\therefore$ & \\
\hline 1. Life Cycle Cost (LCC) & 1 & Sections 1.2 and 4.0 \\
\hline $\begin{array}{l}\text { D. Detaled Instructions for Assessuent of RTP } \\
\text { - Appendix C, November } 14,1997\end{array}$ & (3. & 1) \\
\hline $\begin{array}{l}\text { 2. Provide correct level-1 and mid-level logics and } \\
\text { schedules that support the waste feed delivery. }\end{array}$ & $\mathrm{I}$ & Sections $2.4,3.2$ and 3.3 \\
\hline 3. Provide validatable task duration estimates & $\mathrm{I}$ & Sections $1.2,4.1,4.2$, and 4.3 \\
\hline $\begin{array}{l}\text { 4. Provide confirmable critical path and float } \\
\text { activities not on the path }\end{array}$ & $\mathrm{I}$ & Sections 3.2 and 3.3 \\
\hline 5. Show the ability to meet the June, 2002 hot start & $\mathrm{I}$ & Sections $1.1,3.2,3.3$, and 4.1 \\
\hline $\begin{array}{l}\text { 6. Identify those activities most at risk for meeting } \\
\text { the June, } 2002 \text { hot start }\end{array}$ & 1 & Sections 3.2 and 3.3 \\
\hline $\begin{array}{l}\text { 10. Show the ability of the PHMC to meet all dates } \\
\text { required by the private contractors for the } \\
\text { infrastructure. }\end{array}$ & I & Sections 2.4 and 3.3 \\
\hline $\begin{array}{l}\text { 11. Identify activities most at risk in schedules and } \\
\text { logic diagrams (for infrastructure). }\end{array}$ & I & Sections 3.2 and 3.3 \\
\hline
\end{tabular}


Table B-1. Tank Waste Remediation System Retrieval and Disposal Mission Initial Updated Baseline Summary.

\begin{tabular}{|c|c|c|}
\hline Guidance or Requirement & Status & Implementing Location \\
\hline $\begin{array}{l}\text { 12. Identify projects that directly support } \\
\text { infrastructure, based on project plans. }\end{array}$ & $\mathrm{I}$ & Sections 2.4 and 3.3 \\
\hline $\begin{array}{l}\text { 15. Show the PHMC Team's ability to receive, store } \\
\text { and dispose of immobilized LAW and HLW and } \\
\text { separated cesium from private contractor operations } \\
\text { and to take the responsibilities described in the } \\
\text { following ICDs: }\end{array}$ & & . \\
\hline a. ICD-3 Radioactive Solid Waste & I & Sections 2.4 and 3.3 \\
\hline b. ICD-13 Non-Routine High Level Solid Waste & $\mathrm{I}$ & Sections 2.4 and 3.3 \\
\hline c. ICD-14 IHLW Product & $\mathrm{I}$ & Sections 2.4 and 3.3 \\
\hline d. ICD-15 ILAW Product & $\mathrm{I}$ & Sections 2.4 and 3.3 \\
\hline e. ICD-17 Cs-137 Intermediate Product & $I$ & Sections 2.4 and 3.3 \\
\hline $\begin{array}{l}\text { 16. Show an ability to meet all schedules needed by } \\
\text { the private contractors for receiving immobilized } \\
\text { LAW and HLW and separated cesium. }\end{array}$ & $\mathrm{I}$ & Sections 2.4 and 3.3 \\
\hline $\begin{array}{l}\text { 17. Identify activities most at risk in schedules and } \\
\text { logic diagrams (for ILAW, IHLW, and Cesium). }\end{array}$ & I & Sections 3.2 and 3.3 \\
\hline 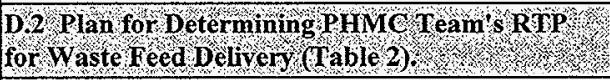 & $8 \%$ & \\
\hline $\begin{array}{l}\text { PHMC provide deliverables necessary to support } \\
\text { RTP, as follows: }\end{array}$ & & $\cdot$ \\
\hline 7. Schedule Baseline & I & Sections $1.2,2.4$ and 3.3 \\
\hline 8. Cost Baseline & I & Section 4.0 \\
\hline 33. PHMC Team Work Packages & I & Section 1.2 .3 .3 and 4.3 \\
\hline $\begin{array}{l}\text { D. } 3 \text { plan for Determining PHM G reams RTP } \\
\text { for Waste Feed Delivery Document Checklist } \\
\text { (Table 3) }\end{array}$ & Sher & 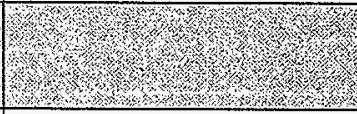 \\
\hline $\begin{array}{l}\text { 16. Plans support post-closure monitoring from } \\
2034 \text { to } 2064 \text {. }\end{array}$ & I & Not applicable to Phase 1 RTP. \\
\hline $\begin{array}{l}\text { 23. Plans are to store separated Tc, entrained HLW } \\
\text { solids, and Sr/TRU products and collect costs of this } \\
\text { transport and storage. }\end{array}$ & I & Section $2.4,3.3,4.1$ and 4.3 \\
\hline $\begin{array}{l}\text { 31. Plans are for upgrades to the evaporator needed } \\
\text { to extend ops. to } 2011 \text { to occur by } 2005 \text {. }\end{array}$ & I & $\begin{array}{l}\text { Sections } 4.1 \text { and } 4.2 .6 \text { (NOTE: } \\
\text { Evaporator Funded by Waste } \\
\text { Management }\end{array}$ \\
\hline
\end{tabular}


Table B-1. Tank Waste Remediation System Retrieval and Disposal Mission Initial Updated Baseline Summary.

\begin{tabular}{|c|c|c|}
\hline Guidance or Requirement & Status & Implementing Location \\
\hline $\begin{array}{l}\text { 58. Plans describe reassessing the timing and needs } \\
\text { for procurement of additional mixer pumps for } \\
\text { DSTs, considering the need dates and availability of } \\
\text { advanced mixer-pump designs. }\end{array}$ & I & Section 3.3 \\
\hline $\begin{array}{l}\text { D. } 5 \text { Draft Plan for Determining RTP for } \\
\text { Infrastructure \& Byproducts Delivery }\end{array}$ & 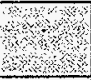 & W. \\
\hline 3. Provide validatable task duration estimates. & I & Sections $1.2,4.1,4.2$, and 4.3 \\
\hline $\begin{array}{l}\text { 4. Provide confirmable critical path and float } \\
\text { activities not on the path. }\end{array}$ & I & Sections 3.2 and 3.3 \\
\hline 5. Show the ability to meet the June, 2002 hot start. & I & Sections $1.1,3.2,3.3$, and 4.1 \\
\hline $\begin{array}{l}\text { D Oraft Plan for Determining RP for } \\
\text { Infrastructure \& Byproducts Delivery, Appendix } \\
\text { B, Programmatic Baseline Checklist. }\end{array}$ & & 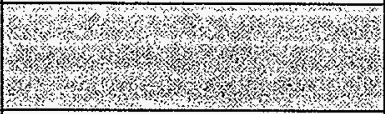 \\
\hline $\begin{array}{l}\text { 30. Indicate that the Infrastructure Level-1 Logic is } \\
\text { consistent with the CDR schedules, the feed delivery } \\
\text { schedules, the Privatization Contractor's schedules, } \\
\text { and other appropriate interface schedules. } \\
(2.1 .1-2.1 .3)\end{array}$ & I & Section $1.2,2.4,3.2$, and 3.3 \\
\hline $\begin{array}{l}\text { 32. Indicate that the WBS is accurate and consistent } \\
\text { with the MYWP. (2.3.1-2.3.2) }\end{array}$ & $\mathrm{I}$ & Sections 2.2 and 2.5 \\
\hline $\begin{array}{l}\text { 34. Show that the critical path contains schedule } \\
\text { contingency. (2:4.3) }\end{array}$ & I & $\begin{array}{l}\text { Section } 2.4,3.2 \text {, and } 3.3 \text { (For } \\
\text { Projects) }\end{array}$ \\
\hline $\begin{array}{l}\text { 38. Provide the Critical Path Diagram Schedule } \\
\text { (CPDS). (4.1.4) }\end{array}$ & I & Section $2.4,3.2$, and 3.3 \\
\hline $\begin{array}{l}\text { D } 8 \text { Draft Plan for Determining RTP for } \\
\text { Infrastructure \& Byproducts Delivery a ppendix } \\
\text { C Infrastructure (Management Baseline) } \\
\text { Chechlist }\end{array}$ & & \\
\hline $\begin{array}{l}\text { 40. Identify the manager assigned to each cost } \\
\text { account (1.1.9) }\end{array}$ & I & $\begin{array}{l}\text { Sections } 1.2 \text { and } 2.4 \text { (Reference } \\
\text { to TBRs) }\end{array}$ \\
\hline $\begin{array}{l}\text { 42. Confirm the Project and SEMP execution plans } \\
\text { are to be developed }(1.2 .4 ; 1.2 .7)\end{array}$ & I & $\begin{array}{l}\text { The execution plans will be } \\
\text { developed as needed }\end{array}$ \\
\hline $\begin{array}{l}\text { 47. Indicate that support is planned/budgeted for RL } \\
\text { preparation of NEPA docs. (1.3.5) }\end{array}$ & I & Sections $1.2,3.3$, and 4.3 \\
\hline 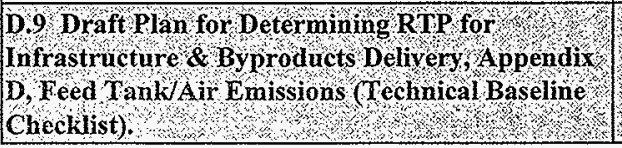 & & \\
\hline
\end{tabular}


Table B-1. Tank Waste Remediation System Retrieval and Disposal Mission Initial Updated Baseline Summary.

\begin{tabular}{|c|c|c|}
\hline Guidance or Requirement & Status & Implementing Location \\
\hline $\begin{array}{l}\text { 59. Show requirements are allocated to functions in } \\
\text { the HSTB and Multi Year Work Plan (MYWP). }\end{array}$ & $\mathrm{I}$ & Sections 2.1 and 2.4 \\
\hline $\begin{array}{l}\text { D } 10 \text { Draft Plan for Determining RTP for } \\
\text { Infrastructure \& Byproducts Delivery, Appendix } \\
\text { E, Infrastructure Feed Tank Transfer } \\
\text { (Programmatic Baseline) Checklist }\end{array}$ & 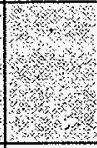 & 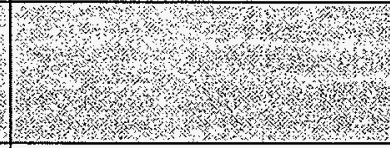 \\
\hline $\begin{array}{l}\text { 74. Indicate that the Infrastructure Level-1 Logic is } \\
\text { consistent with the CDR schedules, the feed delivery } \\
\text { schedules, the Privatization Contractor's schedules, } \\
\text { and other appropriate interface schedules. } \\
(2.1 .1-2.1 .3)\end{array}$ & I & Sections $1.2,2.4,3.2$, and 3.3 \\
\hline $\begin{array}{l}\text { 76. Indicate that the WBS is accurate and consistent } \\
\text { with the MYYP. (2.3.1-2.3.2) }\end{array}$ & $\mathrm{I}$ & Sections 2.2 and 2.5 \\
\hline $\begin{array}{l}\text { 78. Show that the critical path contains schedule } \\
\text { contingency. (2.4.3) }\end{array}$ & I & $\begin{array}{l}\text { Sections 2.4, 3.2, and } 3.3 \text { (For } \\
\text { Projects) }\end{array}$ \\
\hline $\begin{array}{l}\text { 82. Provide the Critical Path Diagram Schedule } \\
\text { (CPDS). (4.1.4) }\end{array}$ & I & Sections $2.4,3.2$ and 3.3 \\
\hline 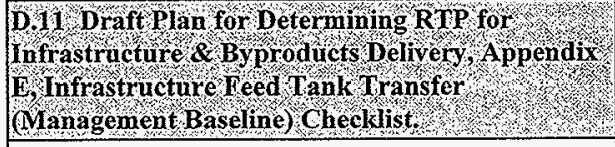 & & 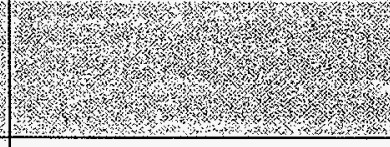 \\
\hline $\begin{array}{l}\text { 84. Identify the manager assigned to each cost } \\
\text { account. (1.1.9) }\end{array}$ & $\mathrm{I}$ & $\begin{array}{l}\text { Sections } 1.2 \text { and } 2.4 \text { (Reference } \\
\text { to TBRs) }\end{array}$ \\
\hline $\begin{array}{l}\text { 86. Confirm the Project and SEMP execution plans } \\
\text { are to be developed. }(1.2 .4 ; 1.2 .7)\end{array}$ & I & $\begin{array}{l}\text { The execution plans will be } \\
\text { developed as needed }\end{array}$ \\
\hline $\begin{array}{l}\text { 91. Indicate that support is planned/budgeted for RL } \\
\text { preparation of NEPA docs. (1.3.5) }\end{array}$ & I & $\begin{array}{l}\text { Sections 1.2,3.3, and 4.3; also } \\
\text { see HNF-1733, Appendix A }\end{array}$ \\
\hline 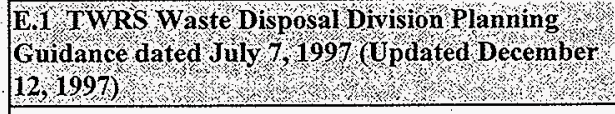 & & \\
\hline Develop a logic-based, resource loaded schedule. & I & Sections $1.2,3.2$, and 3.3 \\
\hline Mandatory completion of approved TPA milestones. & $I$ & Sections $3.2,3.3$, and 3.4 \\
\hline $\begin{array}{l}\text { Baseline schedule for Retrieval will be a subset of } \\
\text { the TWRS Project Master Baseline Schedule. }\end{array}$ & I & Sections 1.2 and 3.3 \\
\hline $\begin{array}{l}\text { Interfaces between elements and external interfaces } \\
\text { must be represented in the PBMS (of which WDD } \\
\text { baseline schedule is a subset). }\end{array}$ & I & Sections 1.2 and 3.3 \\
\hline
\end{tabular}


Table B-1. Tank Waste Remediation System Retrieval and Disposal Mission Initial Updated Baseline Summary.

\begin{tabular}{|c|c|c|}
\hline Guidance or Requirement & Status & Implementing Location \\
\hline $\begin{array}{l}\text { Use the Phase } 1 \text { and } 2 \text { schedules to establish the } \\
\text { WDD schedule. }\end{array}$ & $I$ & $\begin{array}{l}\text { Sections } 3.2 \text { for Phase } 1 \text {; Phase } 2 \\
\text { not covered in RTP schedules }\end{array}$ \\
\hline \begin{tabular}{|l} 
Establish all detailed schedule. consistent with \\
TWRS Level 1 logic.
\end{tabular} & $\mathrm{I}$ & Sections $1.2,2.4$ and 3.3 \\
\hline $\begin{array}{l}\text { Use a code field to facilitate roll-up of detailed } \\
\text { schedules to TWRS Level } 1 \text { and to Level } 0 .\end{array}$ & $I$ & Sections $1.2,3.2$, and 3.3 \\
\hline $\begin{array}{l}\text { Changes to Levels } 1 \text { and } 0 \text { resulting from roll-up } \\
\text { must be put into MYWP. }\end{array}$ & I & $\begin{array}{l}\text { Section } 1.1 \text { (A CR to the } \\
\text { MYWP-Baseline must be } \\
\text { processed) }\end{array}$ \\
\hline $\begin{array}{l}\text { Plan WDD at greatest detail in CY, less in next two } \\
\text { (but sufficient. for logic-driven cost), less in next } 7 \text {, } \\
\text { and sufficient detail beyond ten to define baseline for } \\
\text { entire project. }\end{array}$ & 1 & Section 3.3 \\
\hline $\begin{array}{l}\text { Add sufficient detail in schedules to define critical } \\
\text { path and logical sequences. }\end{array}$ & I & Section 3.2 and 3.3 \\
\hline $\begin{array}{l}\text { Prepared detailed planning describing work scope, } \\
\text { schedule, milestones and budget to complete work } \\
\text { scope. }\end{array}$ & I & Sections $1.0,2.0,3.0$, and 4.0 \\
\hline $\begin{array}{l}\text { Financial staff must define task package control } \\
\text { numbers which allow for proper collection of costs } \\
\text { by schedule activity. }\end{array}$ & I & Section $3.0^{\circ}$ \\
\hline $\begin{array}{l}\text { Waste Retrieval Project will plan for the } 10 \text { Phase } 1 \\
\text { items in paragraph 3.2.1. }\end{array}$ & I & Sections $1.2,2.0,3.0$, and 4.0 \\
\hline $\begin{array}{l}\text { Waste Retrieval Project will assume the } 11 \text { items in } \\
\text { paragraph } 3.2 .2 \text {. }\end{array}$ & $\mathrm{I}$ & $\begin{array}{l}\text { Sections } 1.2,2.0,3.0 \text {, and } 4.0 \\
\text { However, not all items are met, } \\
\text { nor are they Phase } 1\end{array}$ \\
\hline $\begin{array}{l}\text { Waste Feed Delivery will define feed process and } \\
\text { retrieval systems, integrate the waste feed delivery } \\
\text { technical baseline and define Retrieval Project } \\
\text { requirements to interface with HTI and other EM-50 } \\
\text { funded projects. }\end{array}$ & I & $\begin{array}{l}\text { Sections } 2.2,2.4,3.3,4.2 \text {, and } \\
4.3 ; \text { also see HNF } 1901 \text { and } \\
\text { HNF-1881 }\end{array}$ \\
\hline $\begin{array}{l}\text { Complete LAW feed definition, staging plans, } \\
\text { process flowsheets. }\end{array}$ & I & $\begin{array}{l}\text { Sections } 2.2,2.4,3.3,4.2 \text {, and } \\
4.3 ; \text { also see HNF } 1901 \text { and } \\
\text { HNF-1881 }\end{array}$ \\
\hline
\end{tabular}




\section{HNF-1946 Rev. 0}

Table B-1. Tank Waste Remediation System Retrieval and Disposal Mission Initial Updated Baseline Summary.

\begin{tabular}{|l|c|l|}
\hline \multicolumn{1}{|c|}{ Guidance or Requirement } & Status & \multicolumn{1}{|c|}{ Implementing Location } \\
\hline $\begin{array}{l}\text { Include M\&I and Vendor Interface funding and work } \\
\text { scope for Phases } 1 \text { and } 2 \text { in Process. Waste Support } \\
\text { PBS. }\end{array}$ & I & Section 2.2 \\
\hline $\begin{array}{l}\text { Show scope and schedule for WIT and PHMC in the } \\
\text { WDD plans. }\end{array}$ & I & $\begin{array}{l}\text { Sections } 2.1,3.2, \text { and 3.3 (Full } \\
\text { WIT Schedule is not shown in } \\
\text { PHMC Team Schedule) }\end{array}$ \\
\hline
\end{tabular}


HNF-1946 Rev. 0

\title{
APPENDIX C
}

\section{GUIDANCE AND REQUIREMENTS TO}

\section{DELIVERABLES CROSSWALK}

\author{
Level 0 and Level 1 Logics
}

C-i 
HNF-1946 Rev. 0

This page intentionally left blank.

C-ii 


\begin{tabular}{|c|c|c|}
\hline $6+1628-\tau-H$ pur $(M \forall T I) 8+I 6 Z 8-\tau-H)$ & I & 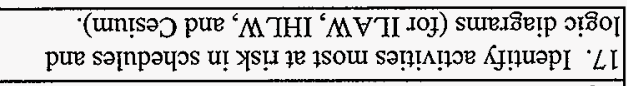 \\
\hline 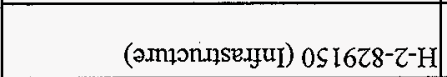 & I & 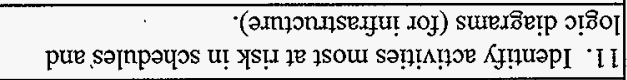 \\
\hline 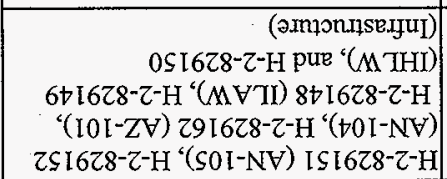 & I & 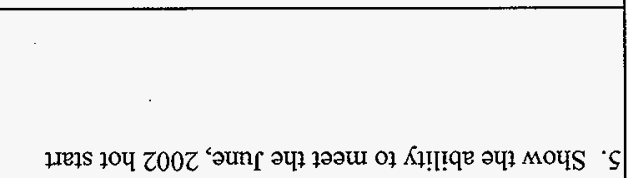 \\
\hline 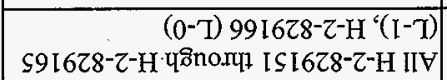 & I & 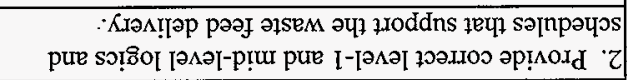 \\
\hline 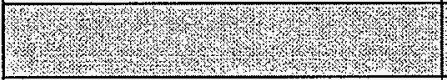 & (x) & 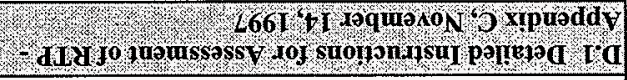 \\
\hline $\operatorname{IIV}$ & I & 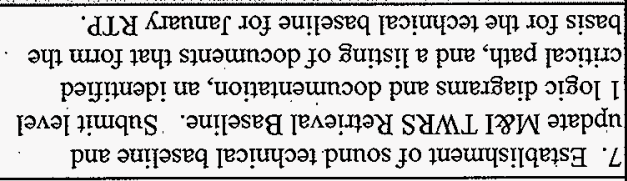 \\
\hline IIV & I & 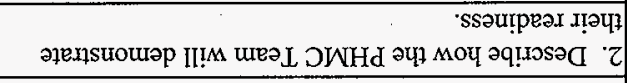 \\
\hline IIV & 1 & 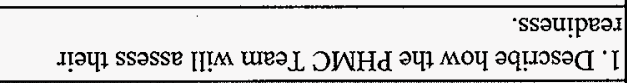 \\
\hline 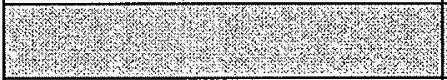 & & 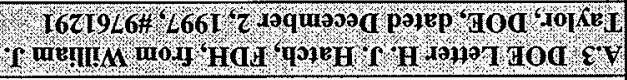 \\
\hline 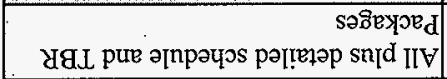 & I & 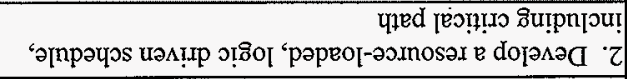 \\
\hline & & 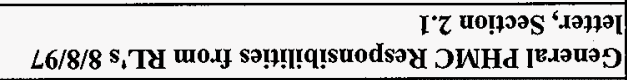 \\
\hline & & 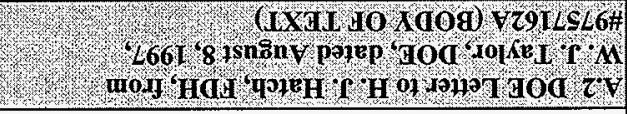 \\
\hline 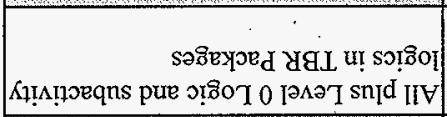 & $\mathrm{I}$ & 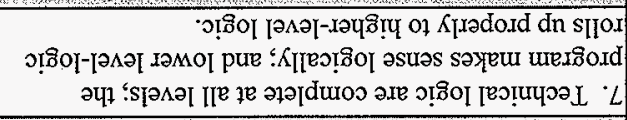 \\
\hline 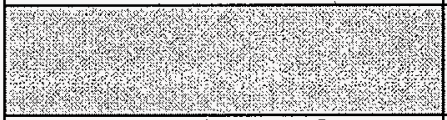 & & 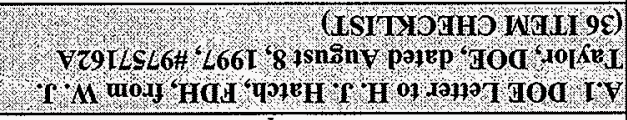 \\
\hline & SRiels & tuəurax!nbəy do วงurp!ng \\
\hline
\end{tabular}

'so! 
Table C-1. Guidance and Requirements to Deliverables Crosswalk - Level 0 and Level 1 Logics.

\begin{tabular}{|c|c|c|}
\hline \multirow{2}{*}{$\begin{array}{l}\text { Guidance or Requirement } \\
\text { D.2 plan for Determining PHMC-Team's RTP for } \\
\text { Waste Feed Delivery (Table } 2 \text { ). }\end{array}$} & \multirow{2}{*}{ Status } & \multirow{2}{*}{ 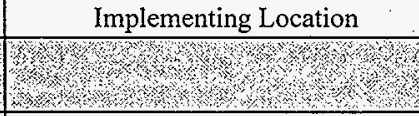 } \\
\hline & & \\
\hline $\begin{array}{l}\text { PHMC provide deliverables necessary to support RTP, } \\
\text { as follows: }\end{array}$ & & \\
\hline 4. Level 0/1 Logic & I & All \\
\hline 13. Multi-level Logic for RTP Package & $\mathrm{I}$ & All \\
\hline $\begin{array}{l}\text { D } 5 \text { Draft Plan for Determining RTP for } \\
\text { Infrastructure \& Byproduct Delivery }\end{array}$ & (2) & W \\
\hline $\begin{array}{l}\text { 2. Provide correct level- } 1 \text { and Level } 0 \text { logics and } \\
\text { schedules that support the infrastructure support. }\end{array}$ & $I$ & $\begin{array}{l}\mathrm{H}-2-829150(\mathrm{~L}-1) \text { and } \mathrm{H}-2-829166 \\
(\mathrm{~L}-0)\end{array}$ \\
\hline $\begin{array}{l}\text { D.7 Draft Plan for Defermining RTP for } \\
\text { Infrastructure \& Byproducts Deliyery, Appendix B, } \\
\text { Programmatic Baseline Checklist. }\end{array}$ & & \\
\hline $\begin{array}{l}\text { 28. Indicate that all activities on logic diagrams are } \\
\text { tiered from contractual or MAR requirements. (1.1.1) }\end{array}$ & I & $\mathrm{H}-2-829150$ \\
\hline $\begin{array}{l}\text { 29. Indicate that activities needed to support } \\
\text { privatization are defined in Level-1 Logic. (1.1.2) }\end{array}$ & $\mathrm{I}$ & All \\
\hline $\begin{array}{l}\text { 30. Indicate that the Infrastructure Level-1 Logic is } \\
\text { consistent with the CDR schedules, the feed delivery } \\
\text { schedules, the Privatization Contractor's schedules, and } \\
\text { other appropriate interface schedules. }(2.1 .1-2.1 .3)\end{array}$ & I & $\mathrm{H}-2-829150$ \\
\hline 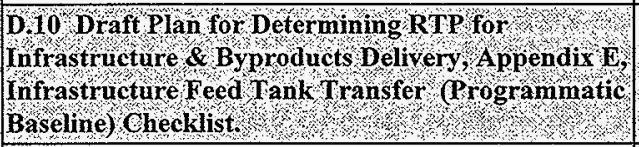 & 60 & \\
\hline $\begin{array}{l}\text { 72. Indicate that all activities on logic diagrams are } \\
\text { tiered from contractual or MAR requirements. (1.1.1) }\end{array}$ & I & $\mathrm{H}-2-829151$ \\
\hline $\begin{array}{l}\text { 73. Indicate that activities needed to support } \\
\text { privatization are defined in Level-1 Logic. (1.1.2) }\end{array}$ & I & H-2-829151 \\
\hline $\begin{array}{l}\text { 74. Indicate that the Infrastructure Level-1 Logic is } \\
\text { onsistent with the CDR schedules, the feed delivery } \\
\text { schedules, the Privatization Contractor's schedules, and } \\
\text { other appropriate interface schedules. }(2.1 .1-2.1 .3)\end{array}$ & I & $\mathrm{H}-2-829151$ \\
\hline
\end{tabular}


HNF-1946 Rev. 0

Table C-1. Guidance and Requirements to Deliverables Crosswalk - Level 0 and Level 1 Logics.

\begin{tabular}{|c|c|c|}
\hline Guidance or Requirement & Status & Implementing Location \\
\hline $\begin{array}{l}\text { E11 TWRSWaste Disposal Division Planning } \\
\text { Guidancedated July } 1997 \text { (Updated December } \\
12,1997)\end{array}$ & & (2) \\
\hline $\begin{array}{l}\text { Establish logic between retrieval, waste proc, } \\
\text { infrastructure, and immobilized tank waste storage and } \\
\text { disposal projects. }\end{array}$ & $\mathrm{I}$. & H-2-829166 (Level-0 Logic). \\
\hline $\begin{array}{l}\text { Ensure logic between privatized and non-priv. } \\
\text { activities through added detail. }\end{array}$ & I & All \\
\hline $\begin{array}{l}\text { Prepared detailed planning describing work scope, } \\
\text { schedule, milestones and budget to complete work } \\
\text { scope. }\end{array}$ & 1 & All \\
\hline
\end{tabular}


HNF-1946 Rev. 0

This page intentionally left blank.

C-4 


\section{Tank Waste Remediation System Retrieval and Disposal Mission Initial Updated Baseline Summary} Level 1 Logics

W. R. Swita

M. R. Lewis

Lockheed Martin Hanford Corporation

M. J. O'Neill

MACTEC

Date Published

January 1998

Prepared by Lockheed Martin Hanford Corporation

Richland, Washington.

Prepared for the U.S. Department of Energy

Fluor Daniel Hanford, Inc.

P.O. Box 1000

Richland, Washington

Hanford Management and Integration Contractor for the

U.S. Department of Energy under Contract DE-AC-0696-RL13200 
HNF-1946 Rev. 0

Volume 2.

LEGAL DISCLAMMER

This report was prepared as an account of work sponsored by an agency of the United States Government. Neither the United States Government nor any agency thereof, nor any of their employees, nor any of their contractors, subcontractors or their employees, makes any warranty, express or implied, or assumes any legal liability or responsibility for the accuracy, completeness, or any third party's use or the results of such use of any information, apparatus, product, or process disclosed, or represents that its use would not infringe privately owned rights. Reference herein to any specific commercial product, process, or service by trade name, trademark, manufacturer, or otherwise, does not necessarily constitute or imply its endorsement, recommendation, or - favoring by the United States Government or any agency thereof or its contractors or subcontractors. The views and opinions of authors expressed herein do not necessarily state or reflect those of the United States Government or any agency thereof.

This report has been reproduced from the best available copy. Avallable in paper copy and microfiche.

Available to the U.S. Department of Energy and its contractors from

U.S. Department of Energy

Office of Scientific and Technical Information (OSTI)

P.O. Box 62

Oak Ridge, TN 37831

(615) 576-8401

Available to the public from the U.S. Department of Commerce National Technical Information Service (NTIS)

5285 Port Royal Road

Springfield, VA 22161

(703) 487-4650

Printed in the United Statos of Americe

DISCLM-1.CHP (8-95) 


\section{HNF-1946 Rev. 0 \\ VOLUME 2 LEVEL 1 LOGICS}

\section{LOW-ACTIVITY WASTE FEED LEVEL 1 LOGICS}

Figure A-1 LAW 1st Feed Batches, Tank 241-AN-105

Figure A-2 LAW 2nd Feed Batches, Tank 241-AN-104

Figure A-3 LAW 3rd Feed Batches, Tank 241-AW-101

Figure A-4 LAW 4th Feed Batches, Tank 241-AN-103

Figure A-5 LAW 5th Feed Batches, Tanks 241-AP-101 \& 241-AW-104

Figure A-6 LAW 6th Feed Batches, Tank 241-AY-101

Figure A-7 LAW 7th \& 8th Feed Batches, Tank 241-AN-107

Figure A-8 LAW 9th Feed Batches, Tank 241-AN-102

Figure A-9 LAW 10th Feed Batches, Tank 241-AN-106 


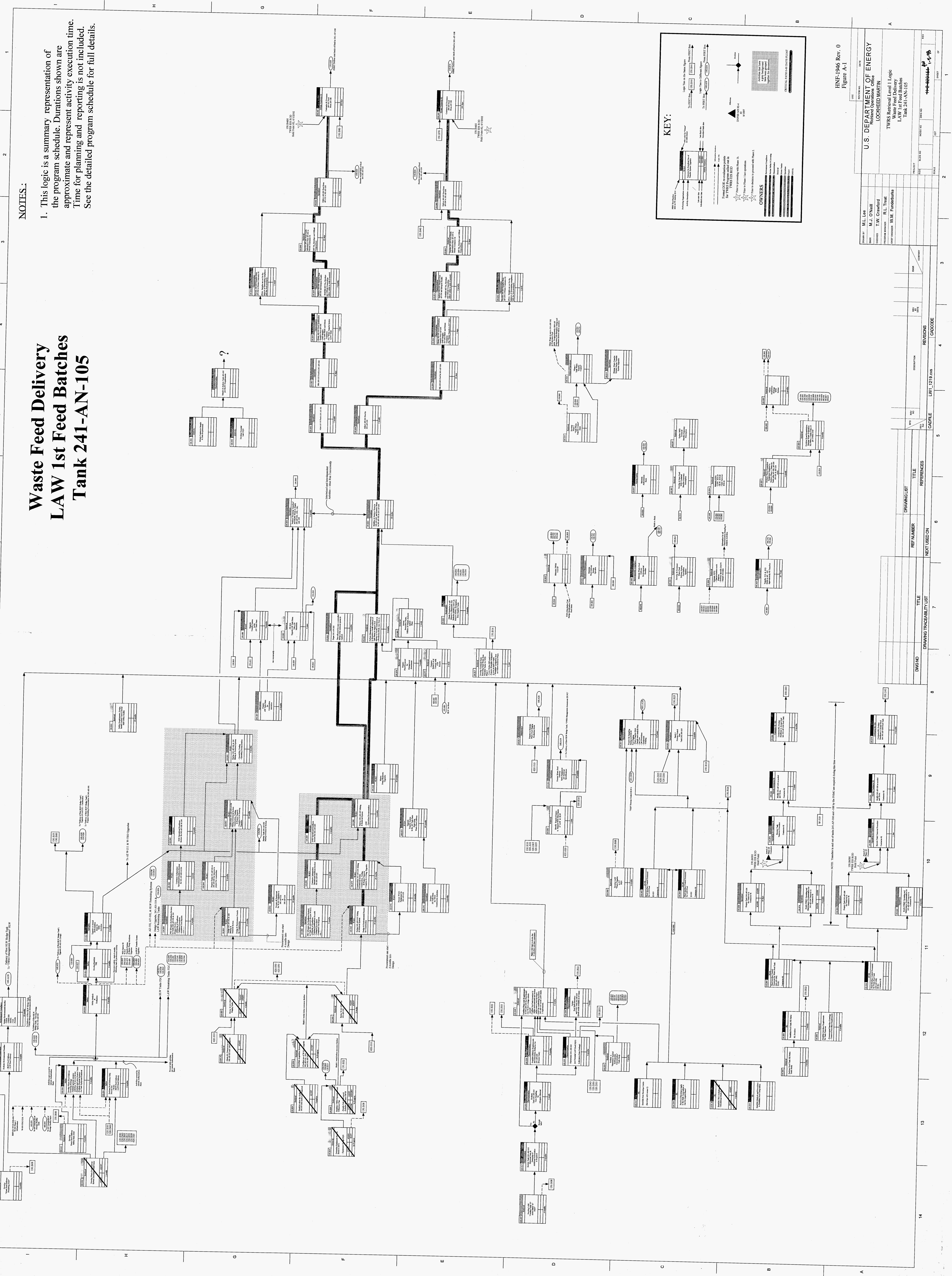



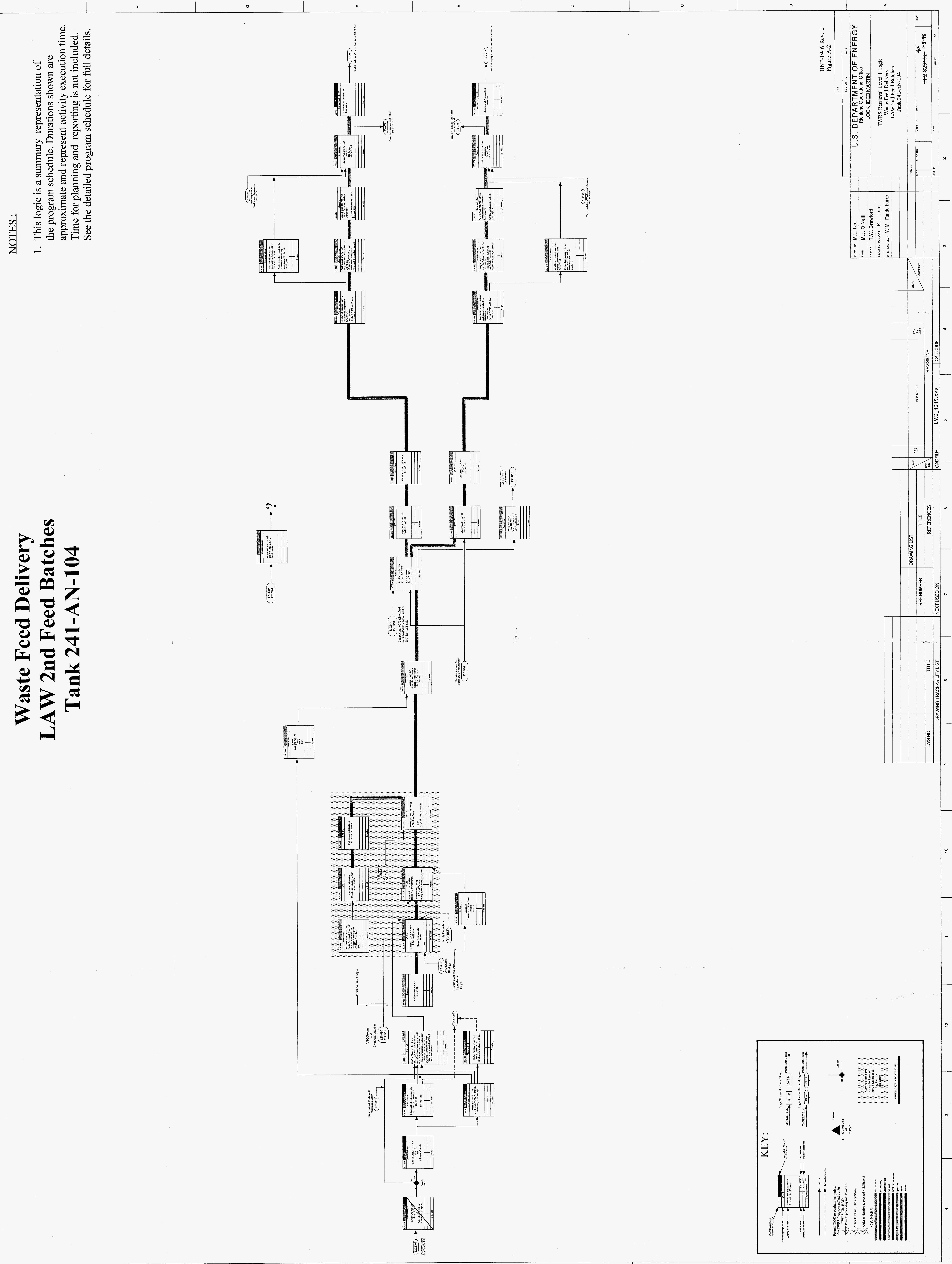


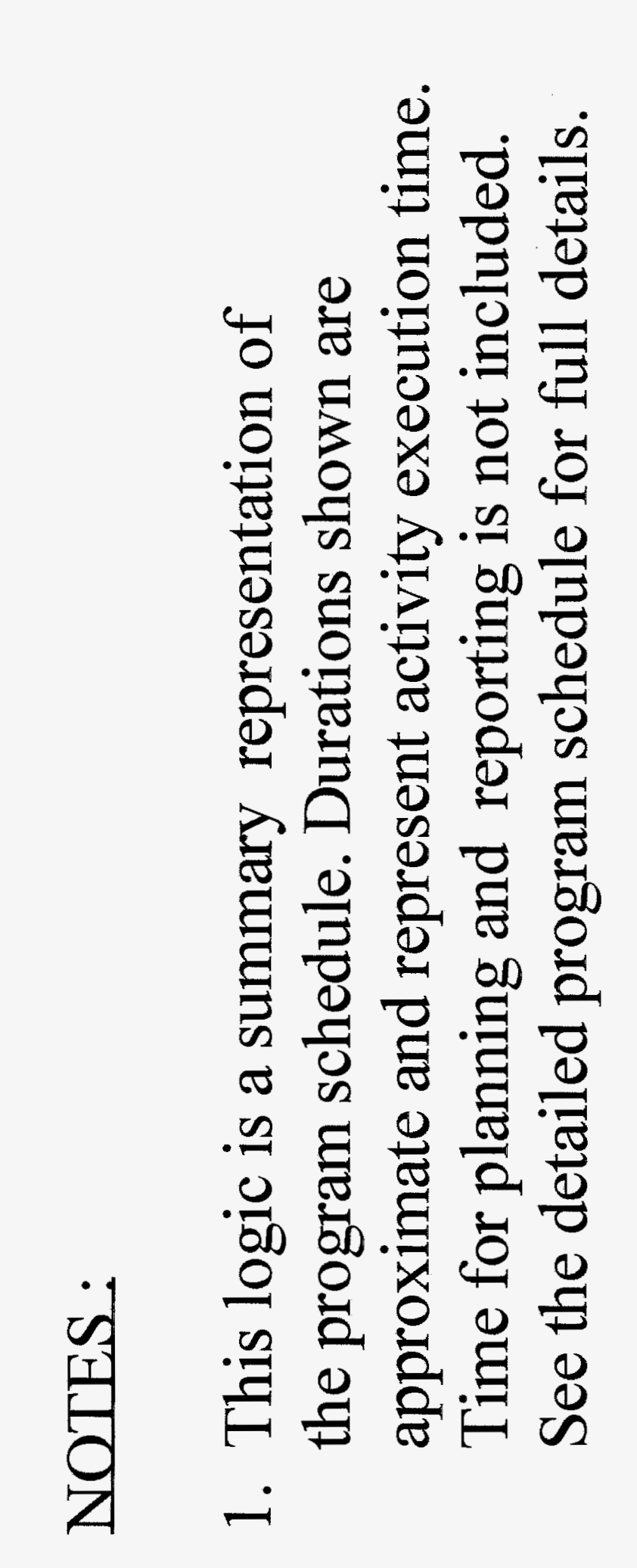

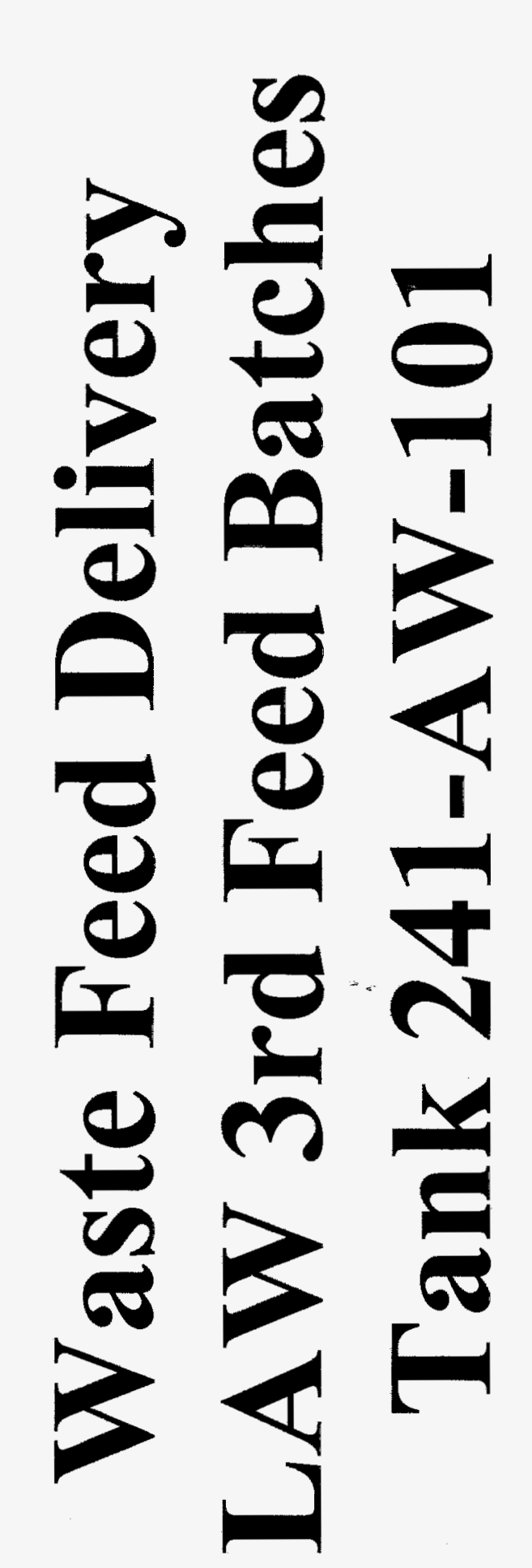

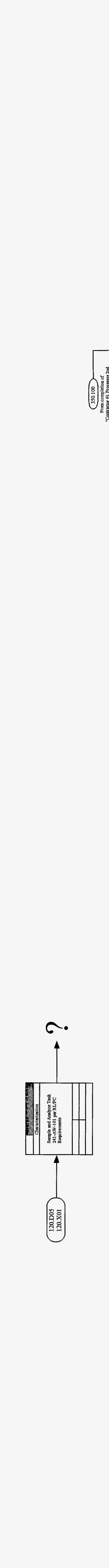

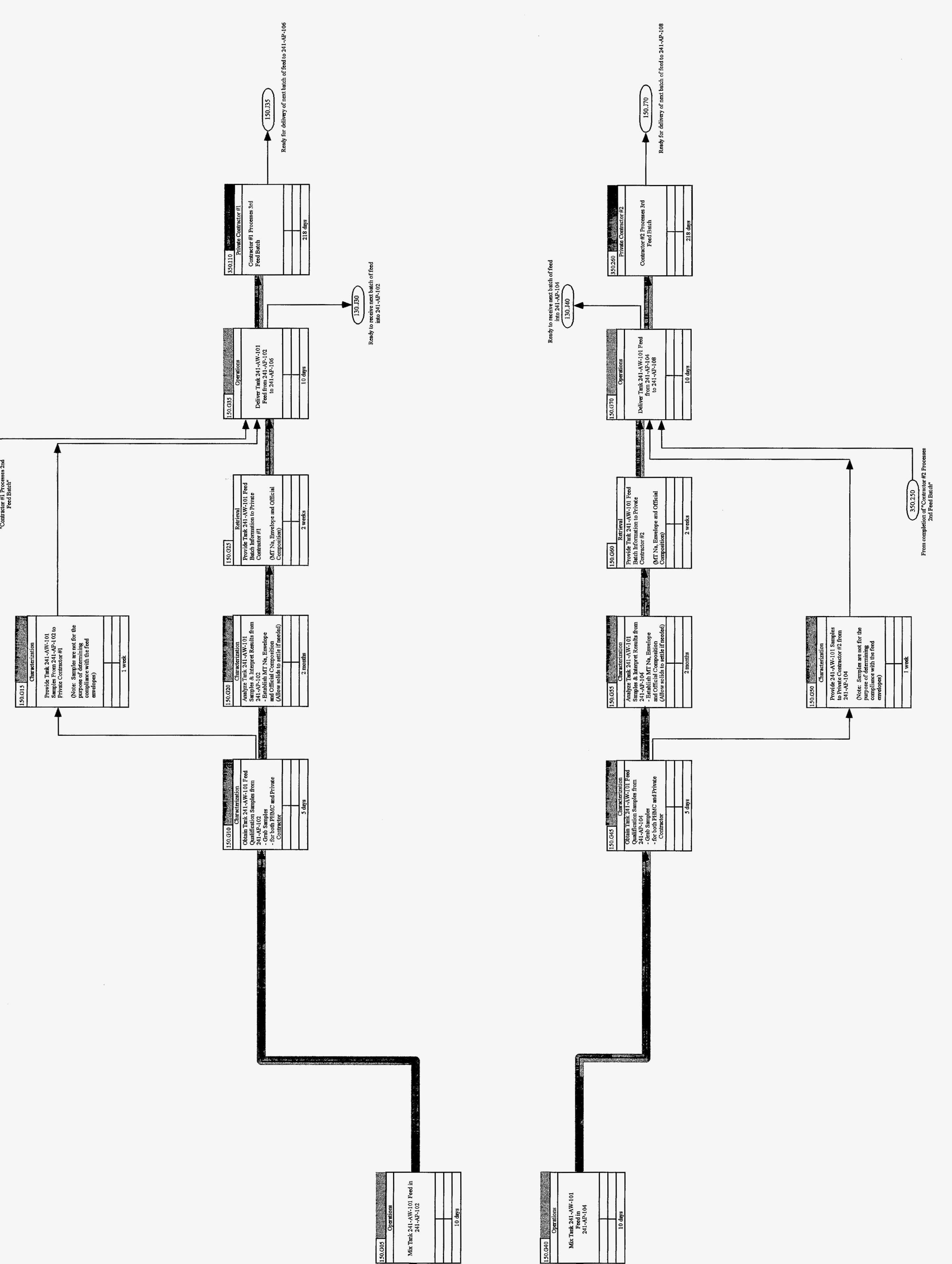

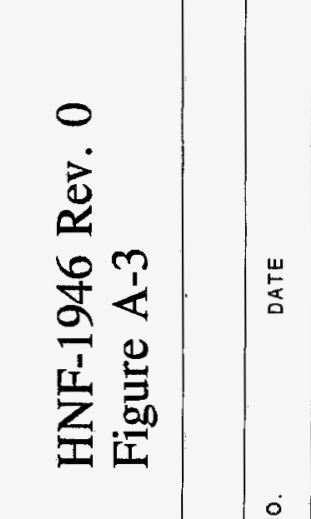

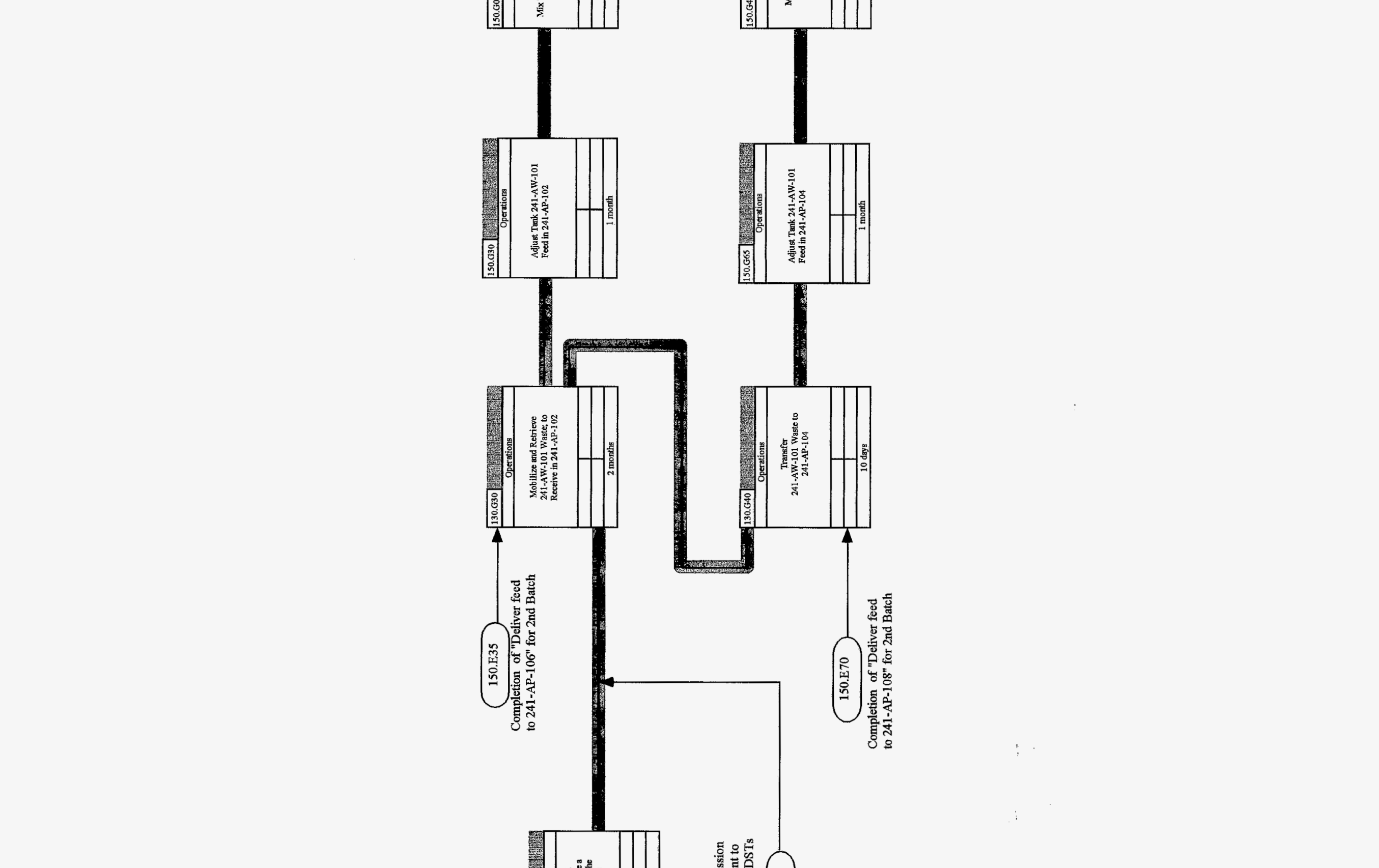

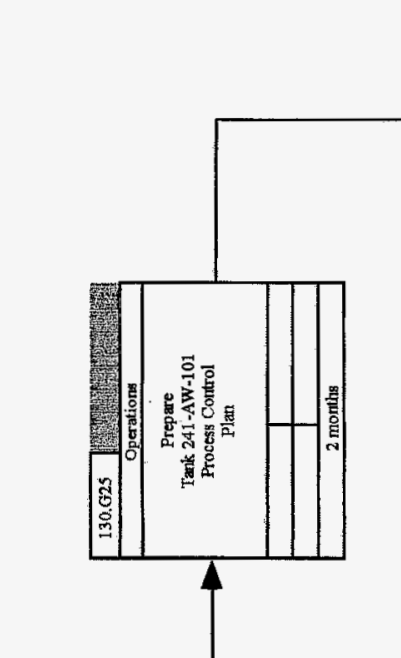

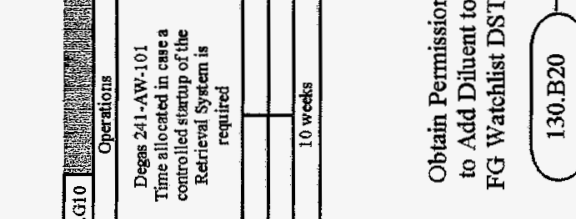

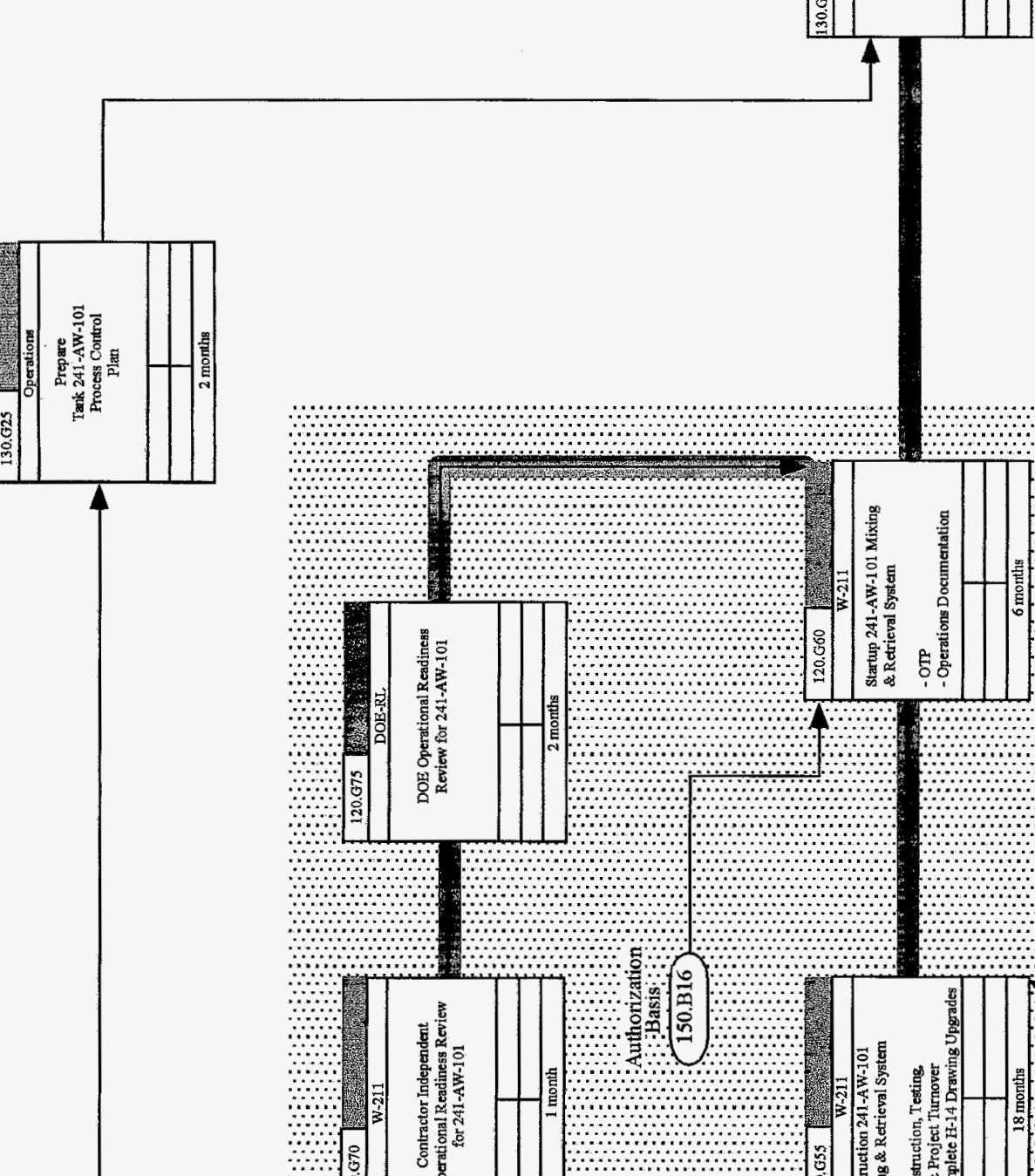

$\frac{1}{1}$

III)

阅!

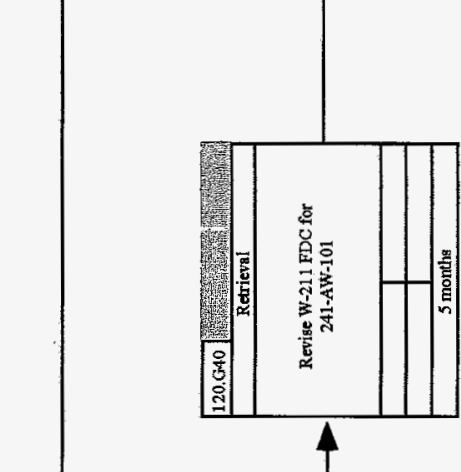
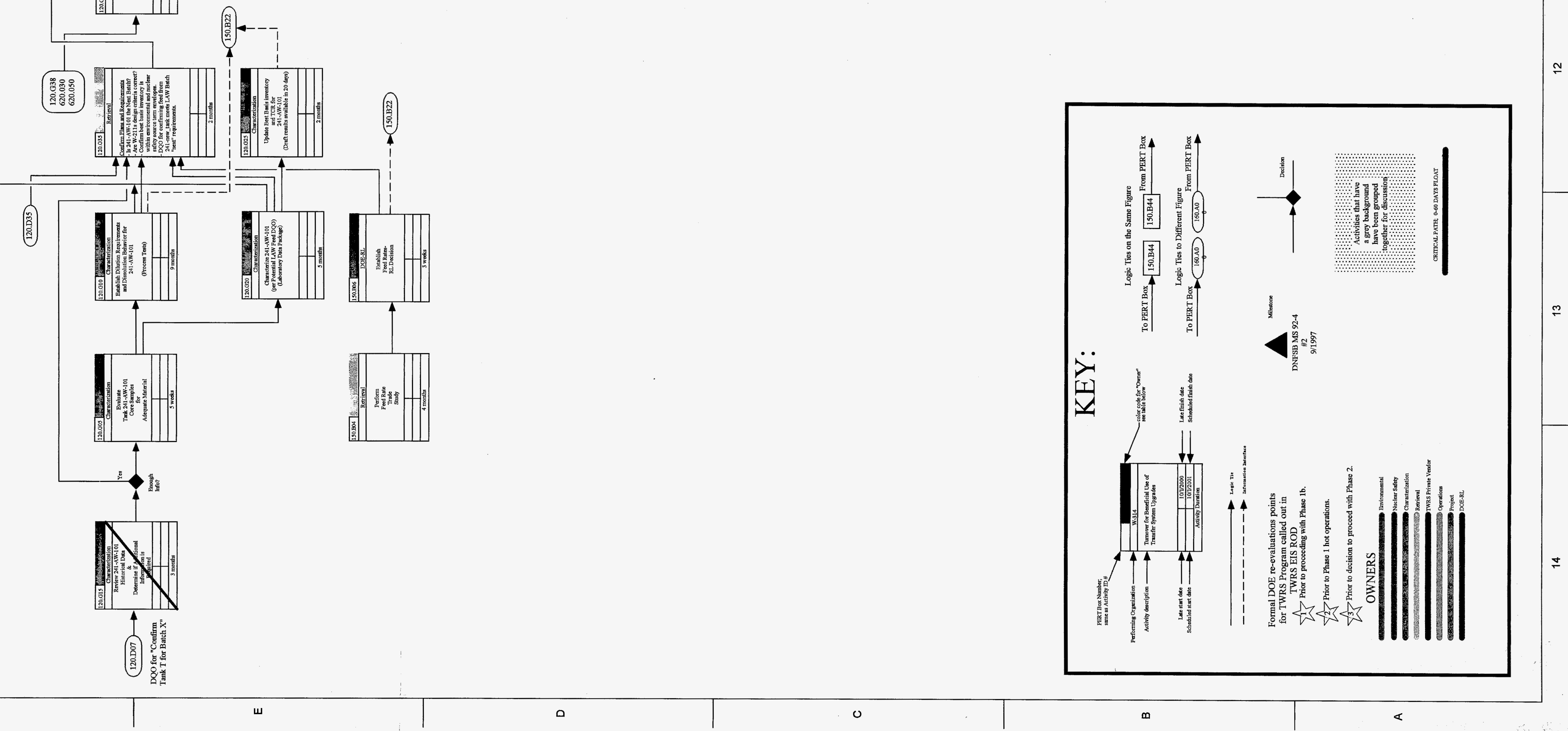


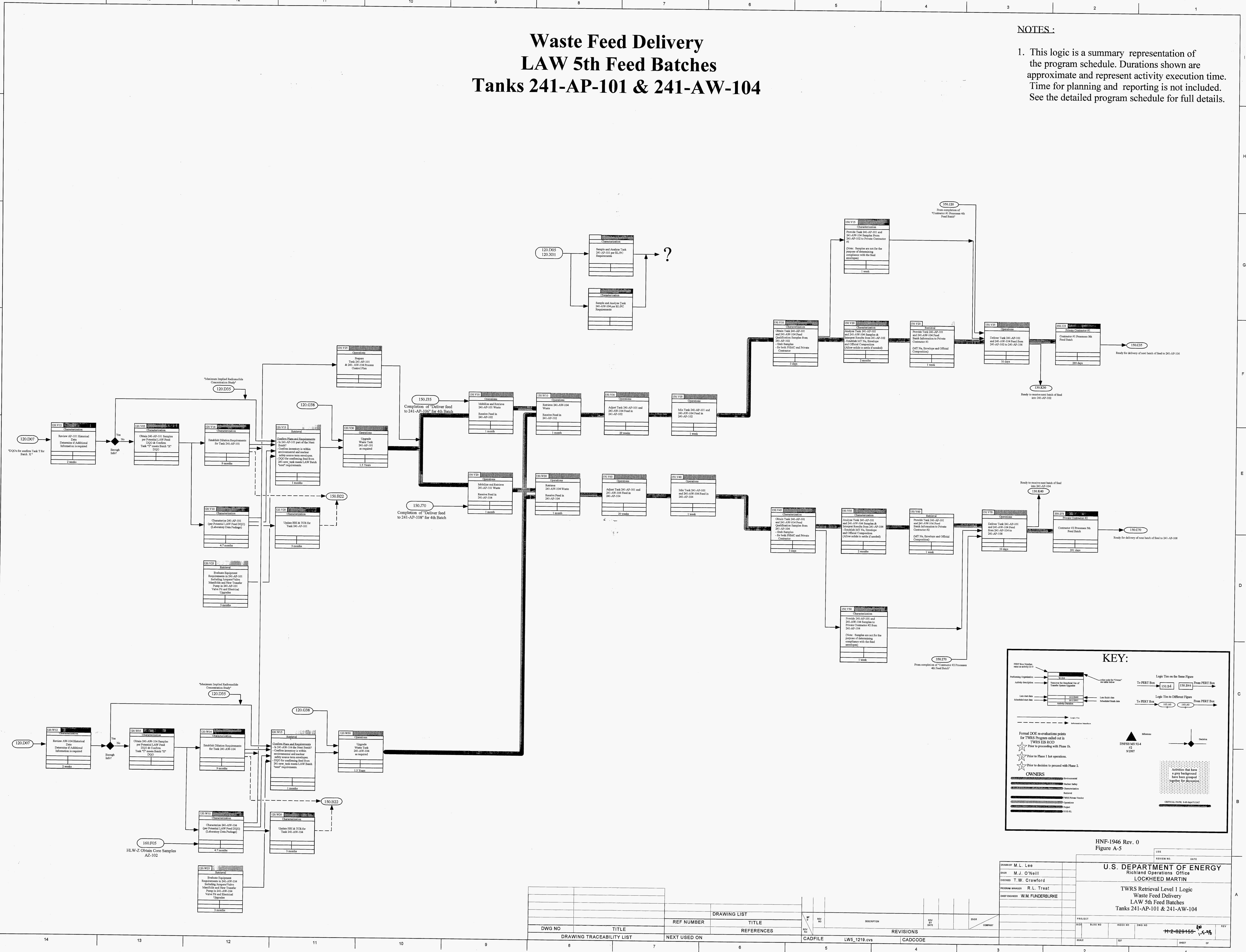




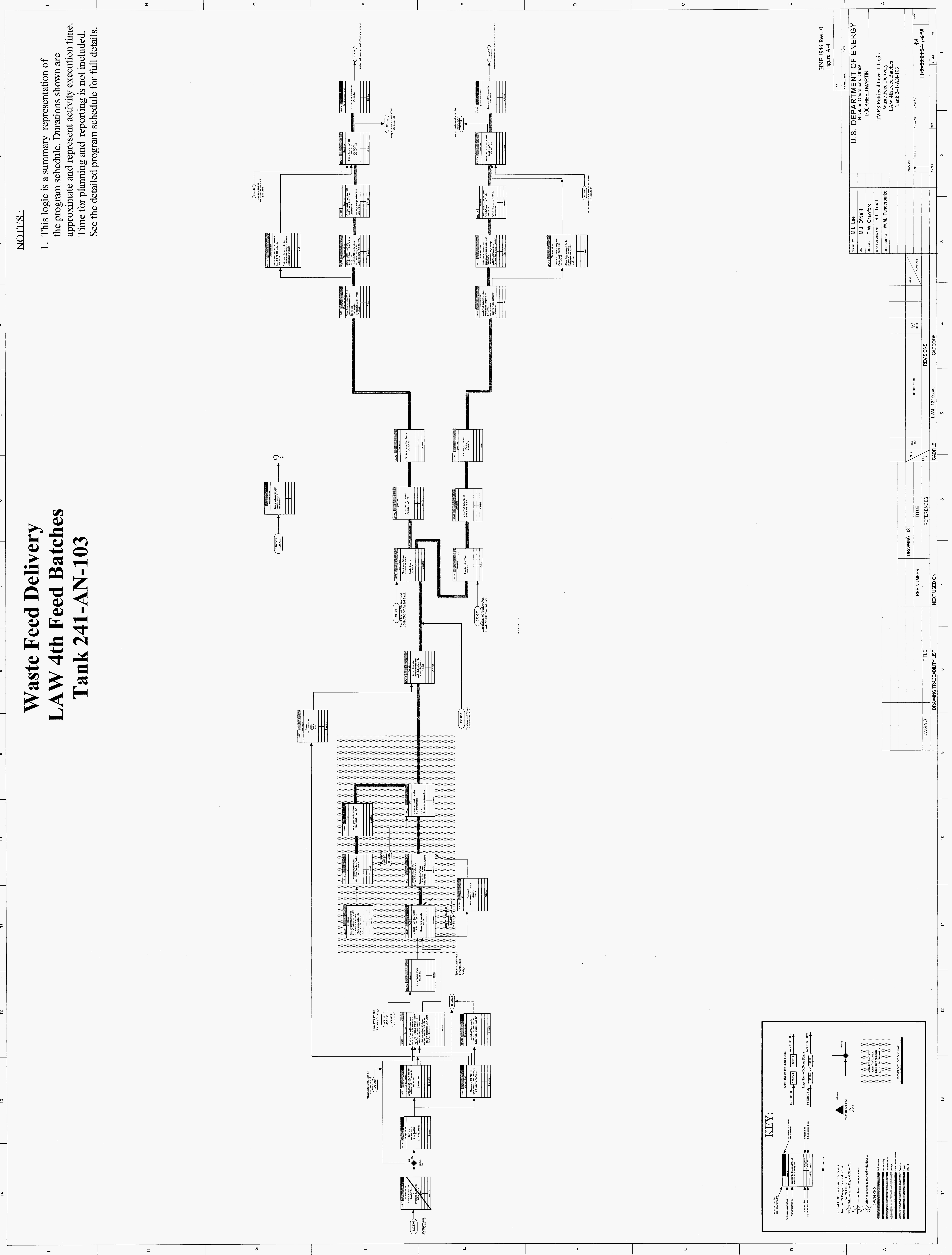



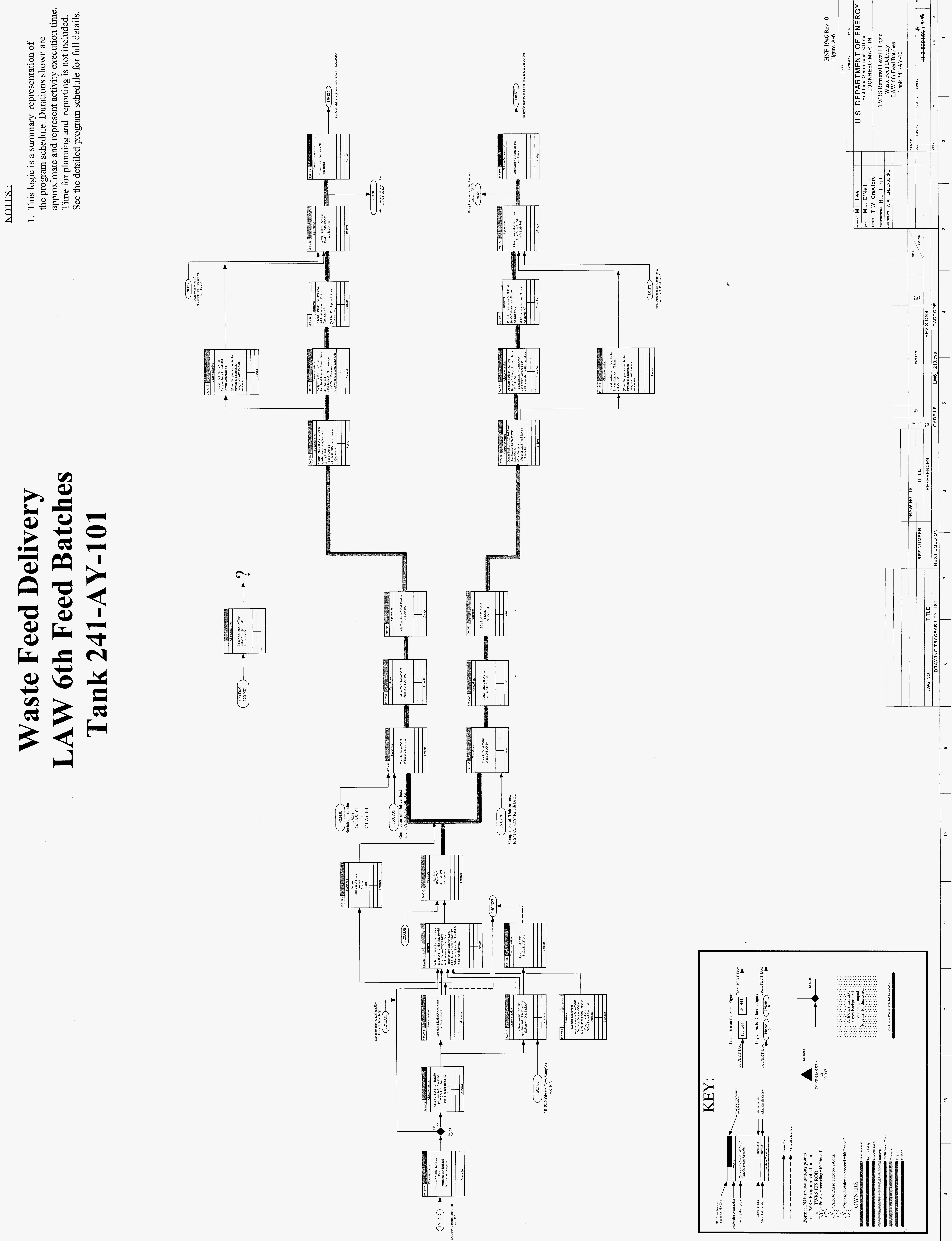

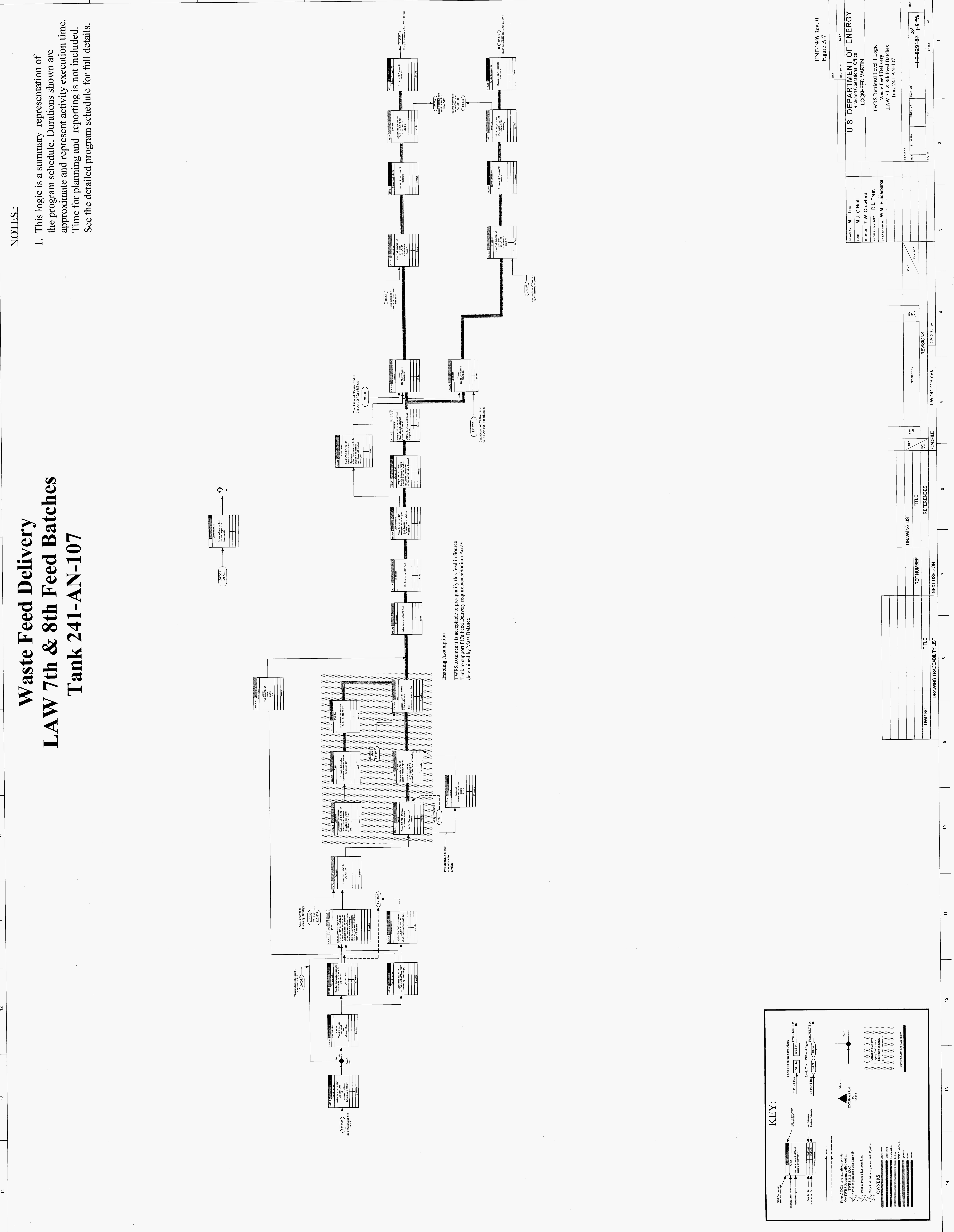

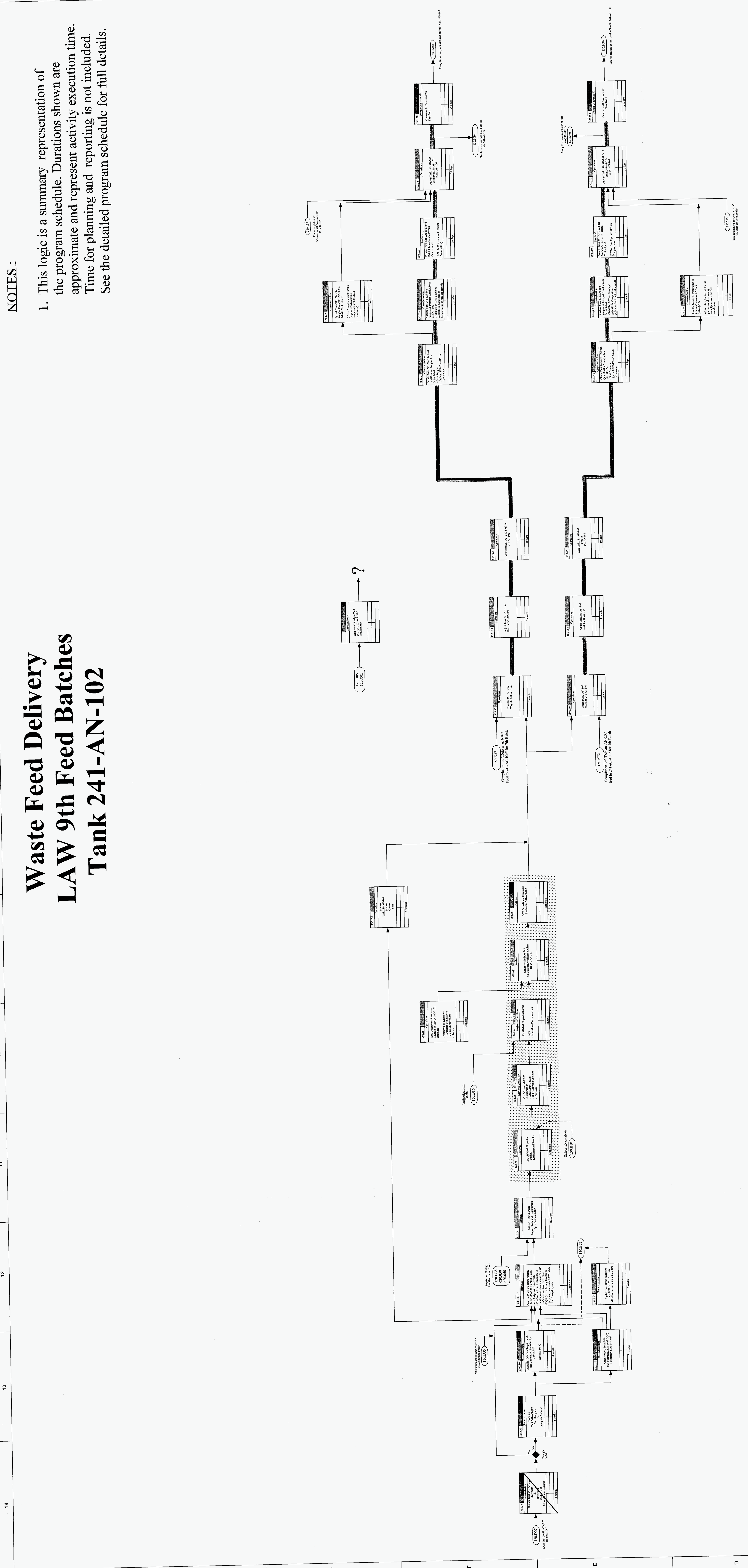

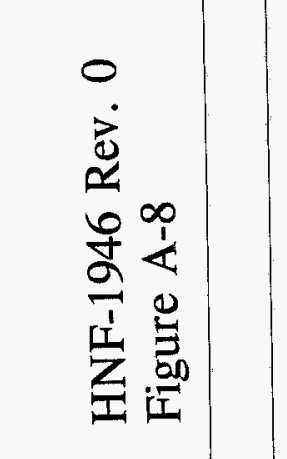

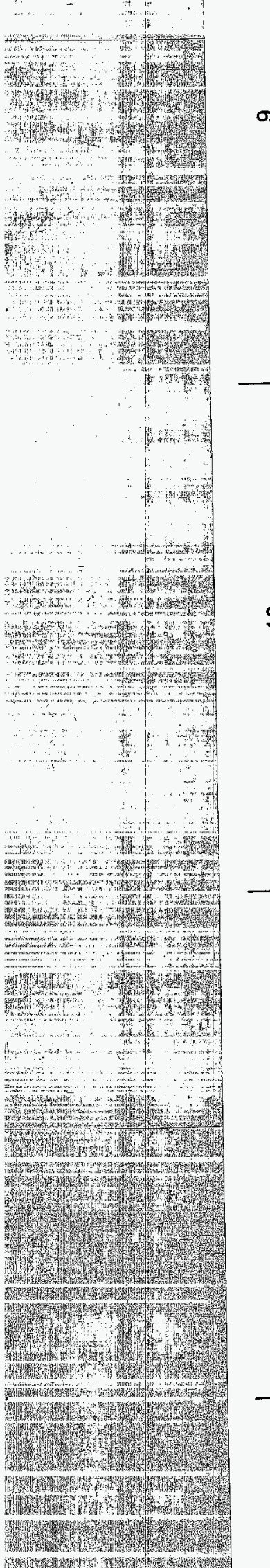




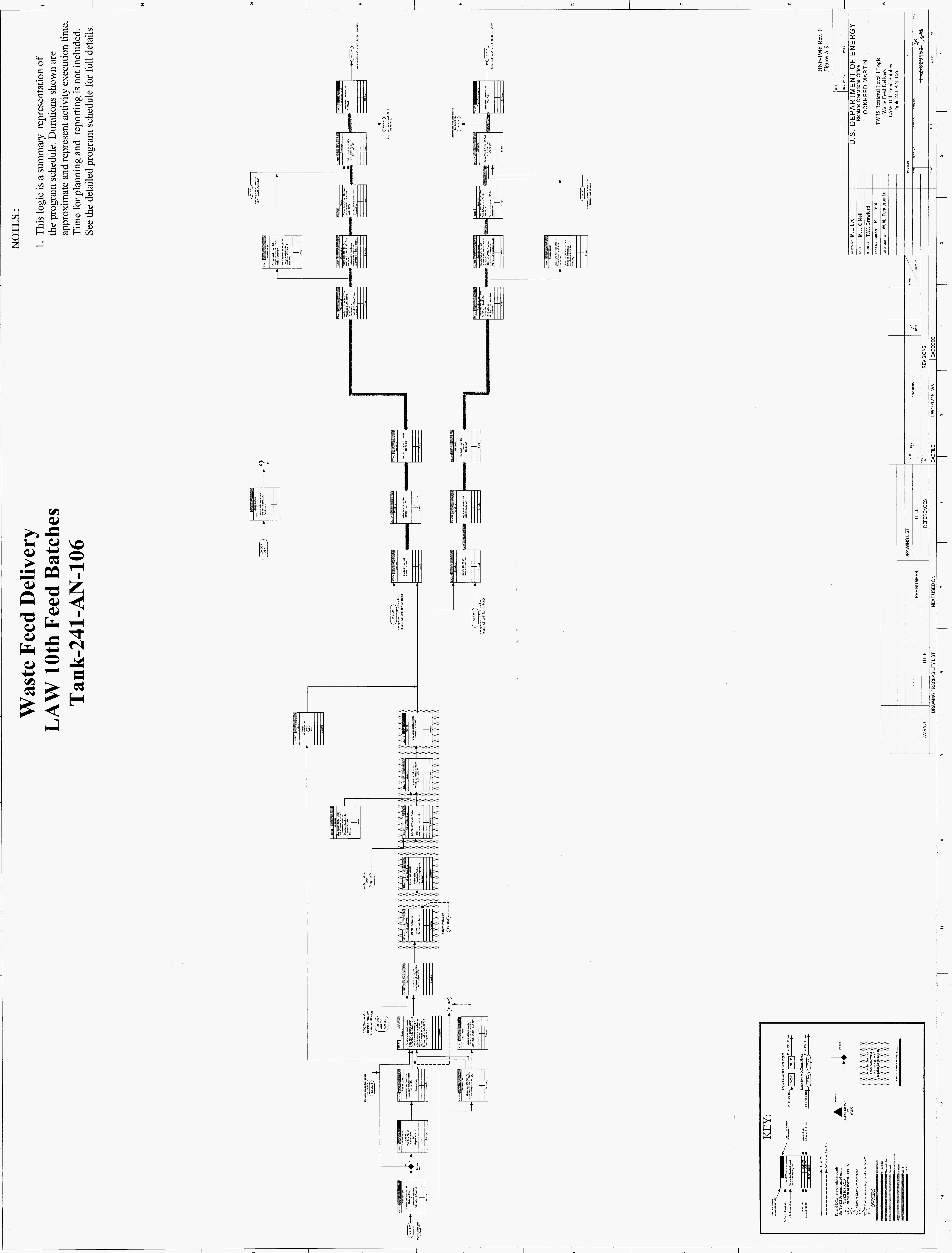


HNF-1946

Volume 3

Rev. 0

UC-510

\section{Tank Waste Remediation System Retrieval and Disposal Mission Initial Updated Baseline Summary Level 1 Logics}

W. R. Swita

M. R. Lewis

Lockheed Martin Hanford Corporation

M. J. O'Neill

MACTEC

Date Published.

January 1998

Prepared by Lockheed Martin Hanford Corporation

Richland, Washington

Prepared for the U.S. Department of Energy

Fluor Daniel Hanford, Inc.

P.O. Box 1000

Richland, Washington 
HNF-1946 Rev. 0

Volume 3

LEGAI. DISCLAMMER

This report was prepared as an account of work sponsored by an agency of the United States Government. Neither the United States Government nor any agency thereof, nor any of their employees, nor any of their contractors, subcontractors or their employees, makes any warranty, express or implied, or assumes any legal liability or responsibility for the accuracy, completeness, or eny third party's use or the results of such use of any information, apparatus, product, or process disclosed, or represents that its use would not infringe privately owned rights. Reference herein to any specific commercial product, process, or service by trade name, trademark, manufacturer, or otherwise, does not necessarily constitute or imply its endorsement, recommendation, or favoring by the United States Government or any agency thereof or its contractors or subcontractors. The views and opinions of authors expressed herein do not necessarily state or reflect those of the United States Government or any agency thereof.

This report has been reproduced from the best available copy. Available in paper copy and microfiche.

Available to the U.S. Department of Energy and its contractors from

U.S. Department of Energy

Office of Scientific and Technical Information (OSTI)

P.O. Box 62

Oak Ridge, TN 37831

(615) 576-8401

Avalable to the public from the U.S. Department of Commerce National Technical information Service (NTIS)

5285 Port Royal Road

Springfield, VA 22161

(703) $487-4650$

Printod in the United Stotes of Americs

DISCLM-1.CHP (8-95) 
HNF-19.46 Rev. 0

VOLUME 3 LEVEL 1 LOGICS

LOW-ACTIVITY WASTE FEED LEVEL-1 LOGICS

Figure A-10 LAW 11th Feed Batches, Tank 241-SY-101

Figure A-11 LAW 12th Feed Batches, Tank 241-SY-103

HIGH-LEVEL WASTE FEED LEVEL 1 LOGICS

Figure A-12 HLW 1st \& 2nd Feed Batches, First Tank 241-AZ-101

Figure A-13 HLW 3rd \& 4th Feed Batches, First Tank 241-AZ-102

Figure A-14 HLW 5th - 9 th Feed Batches, 241-AY-102

Figure A-15 HLW 10th - 12th Feed Batches, Tank 241-C-104

IMMOBILIZED LOW-ACTIVITY WASTE LEVEL 1 LOGICS

Figure A-16 Immobilized Waste ILAW

IMMOBILIZED HIGH-LEVEL WASTE LEVEL 1 LOGICS

Figure A-17 Immobilized Waste IHLW

INFRASTRUCTURE PHASE 1 LEVEL 1 LOGICS

Figure A-18 Phase 1 Privatization Support 


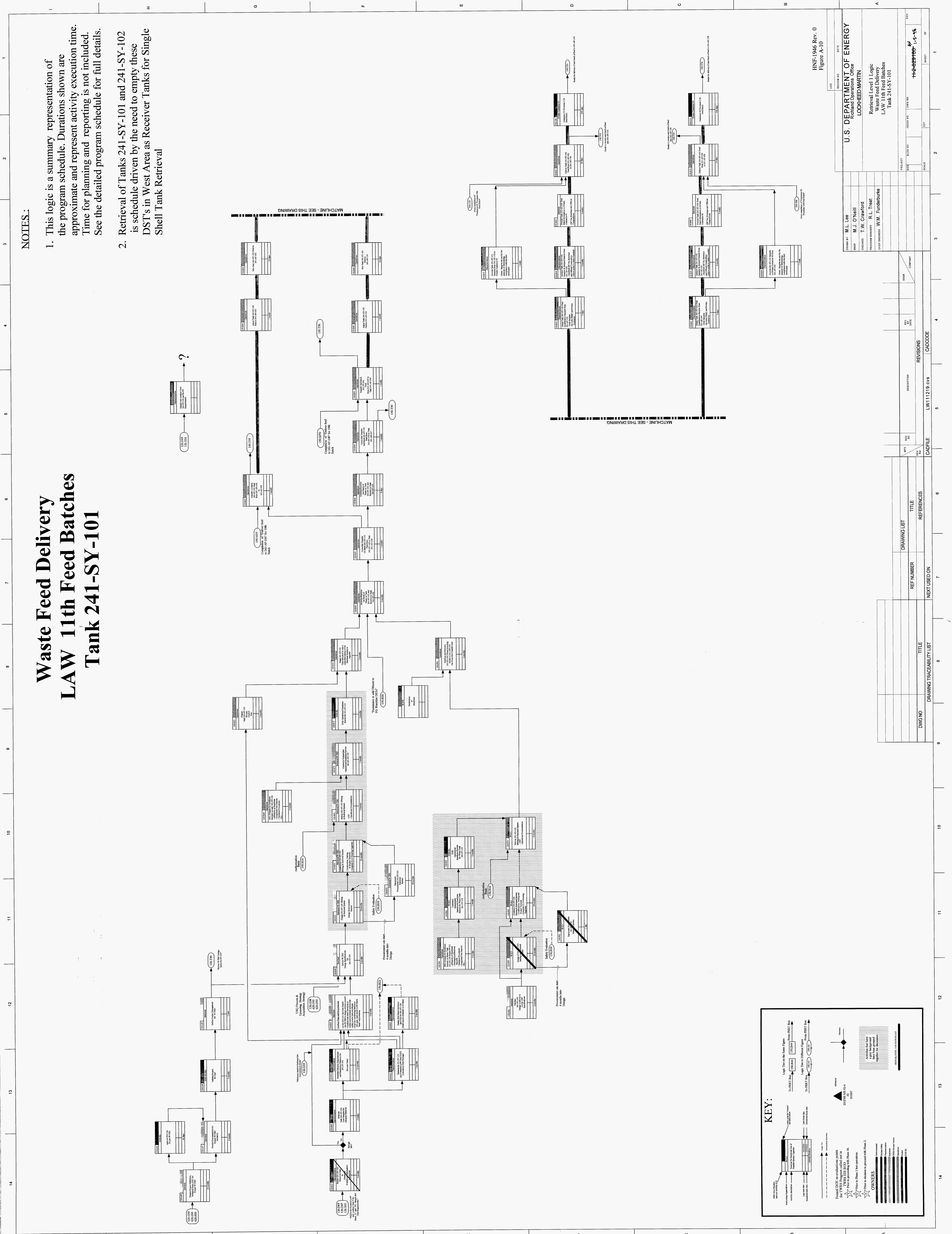




\section{Waste Feed Delivery}

Tank 241-SY-103
LAW 12th Feed Batches

NOTES:

This logic is a summary representation of the program schedule. Durations shown are approximate and represent activity execution time. See the detailed program schedule for full details.

2. Retrieval of Tank 241-SY-103 is schedule driven by the need to empty this DST in West Area Retrieval.
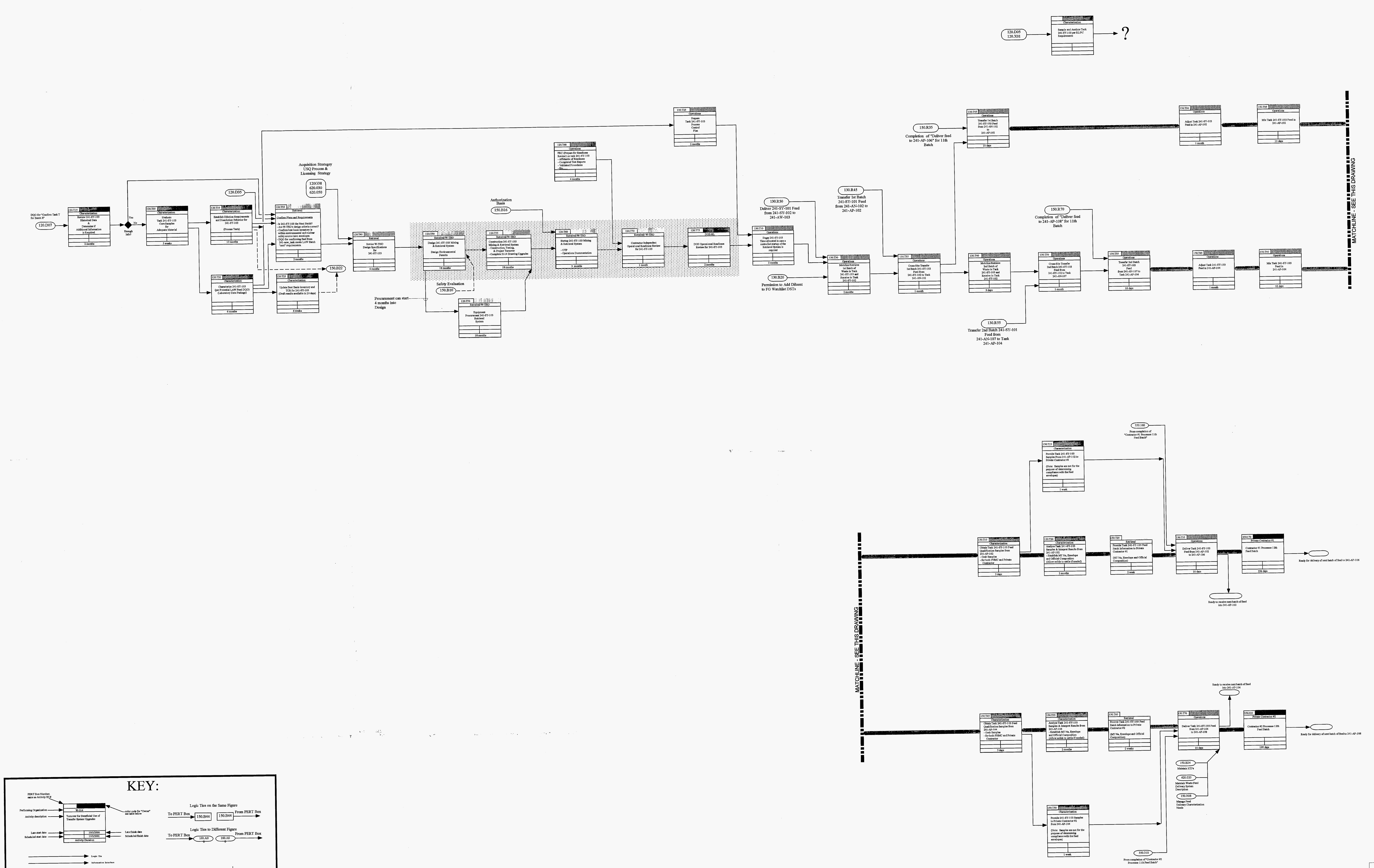

HNE-1946 Rev.
Figure $A-11$ 


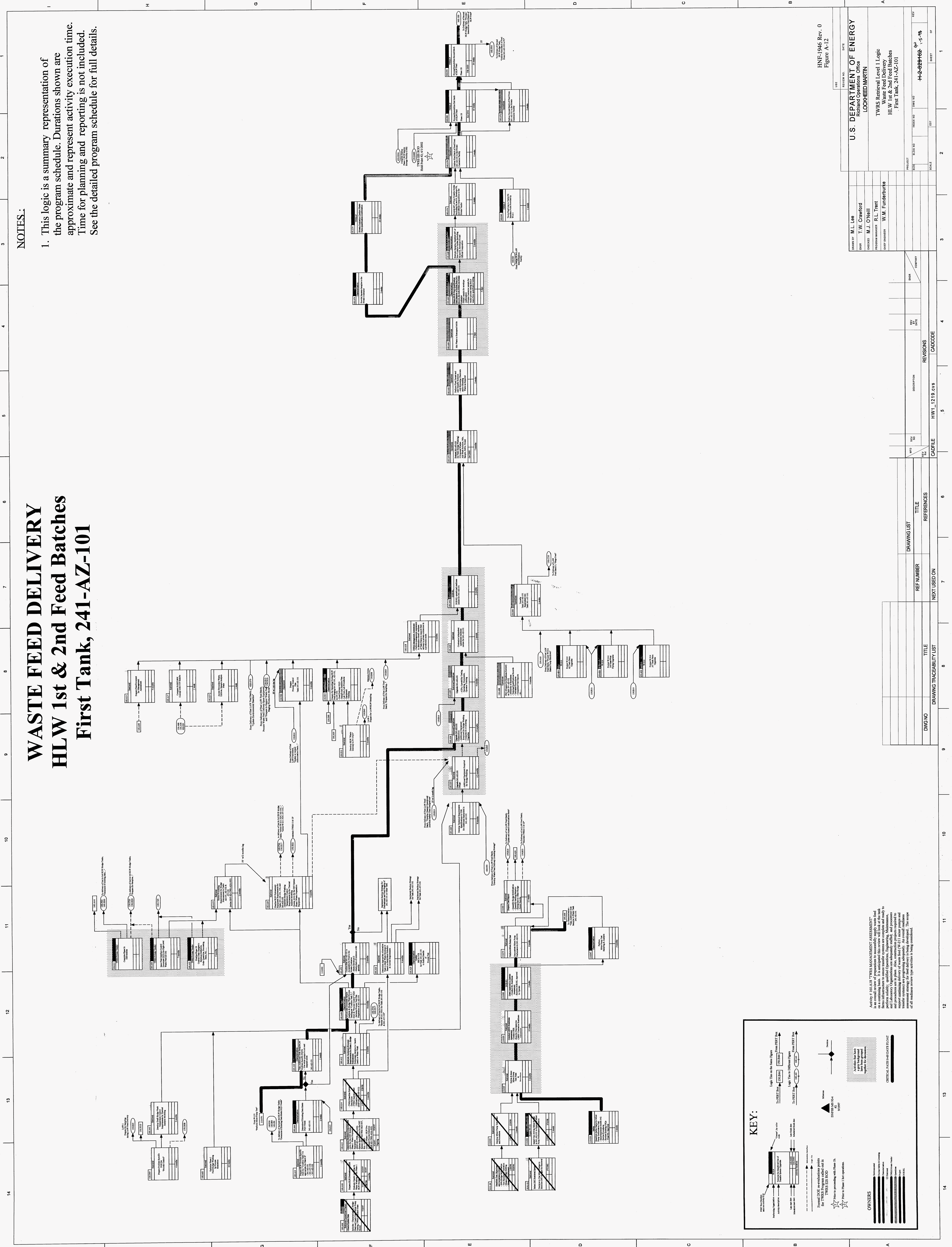




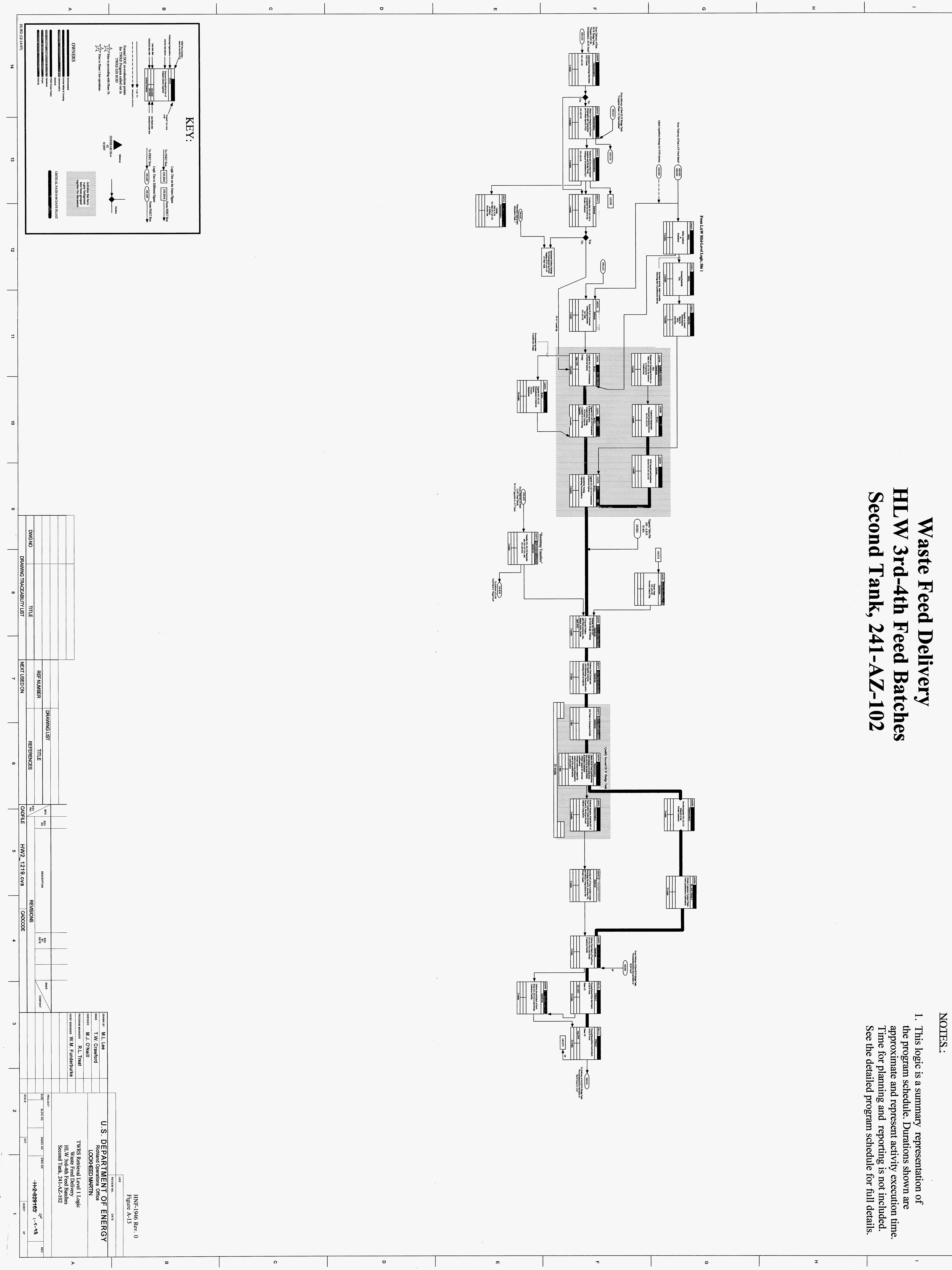




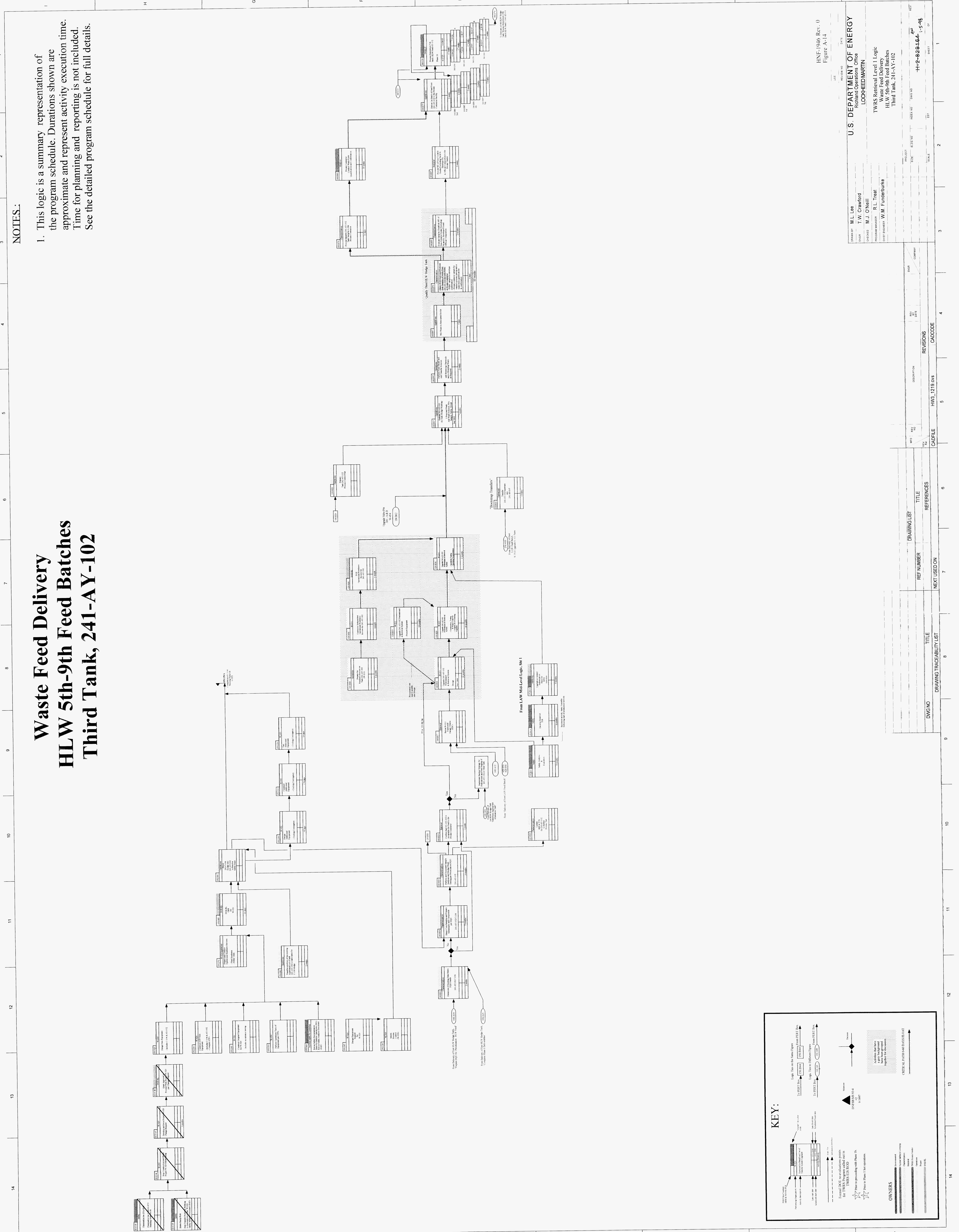




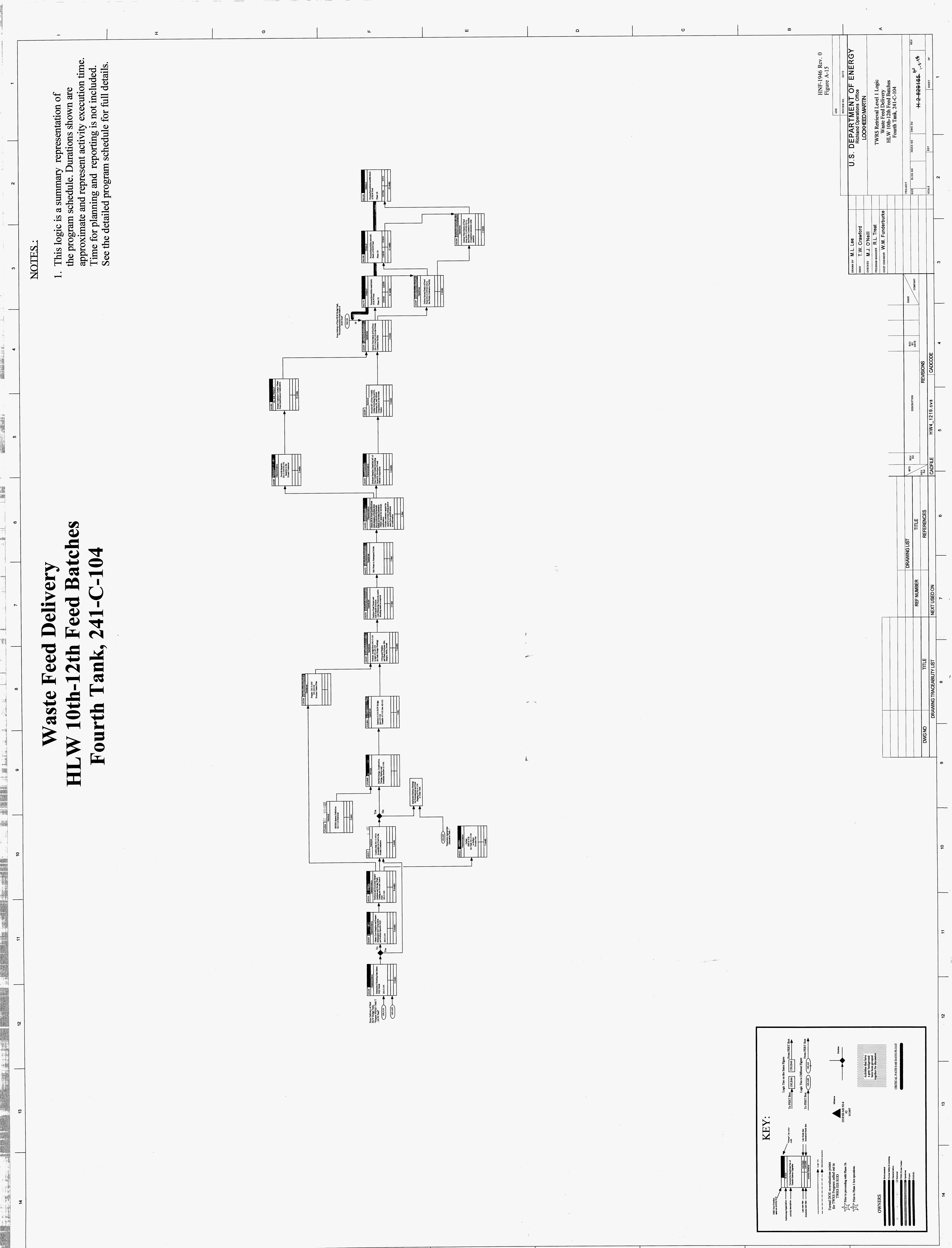




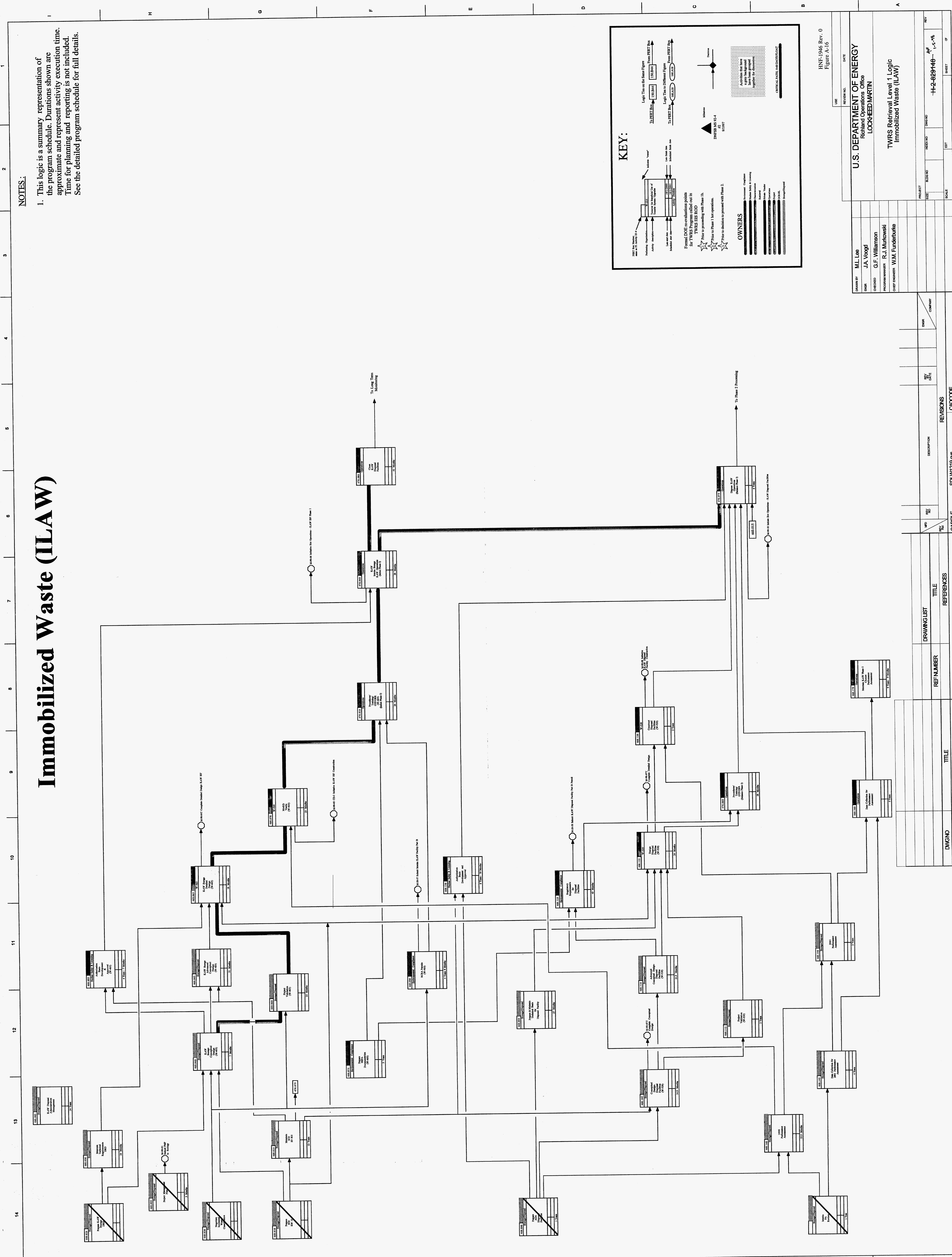




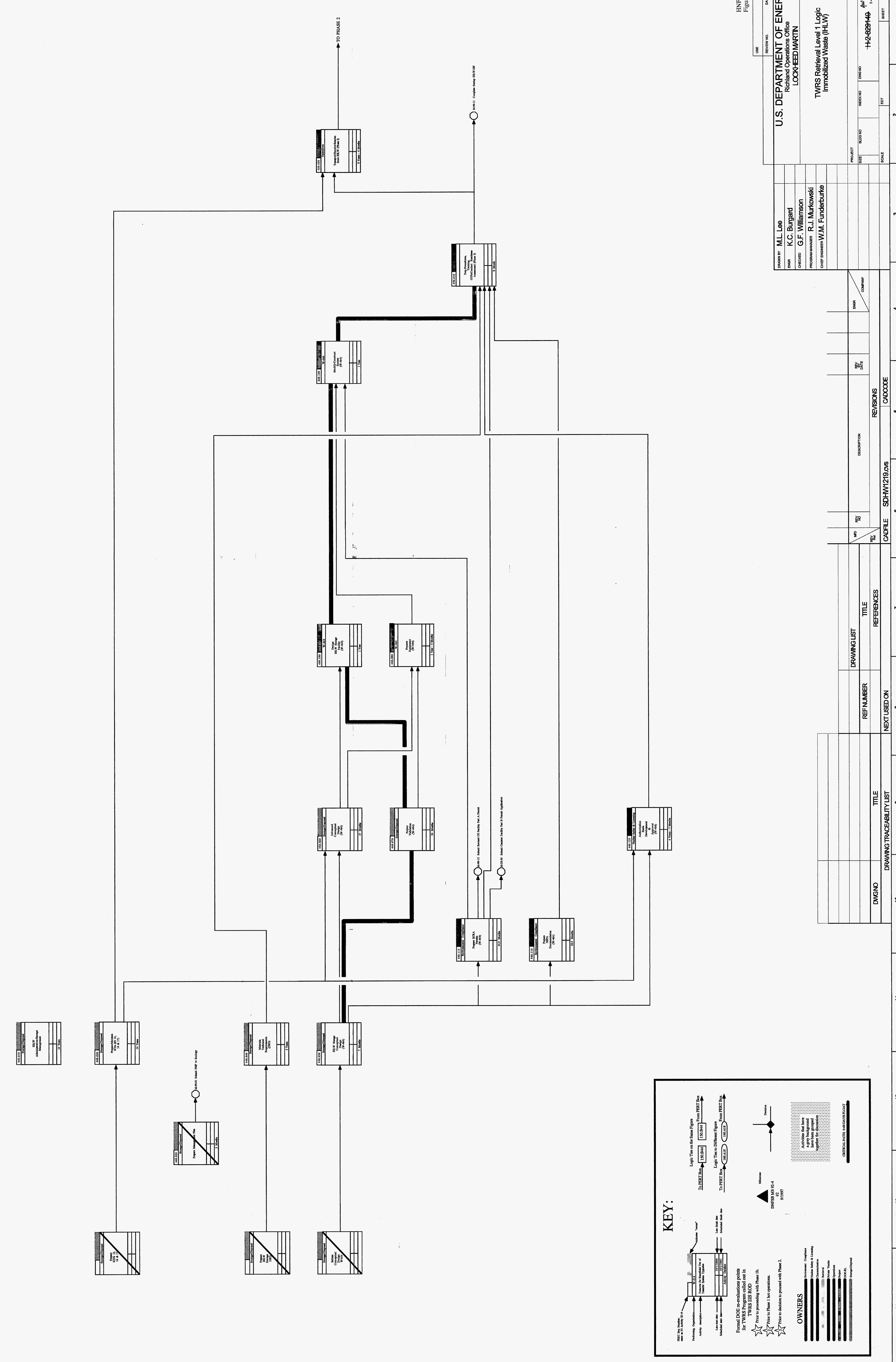




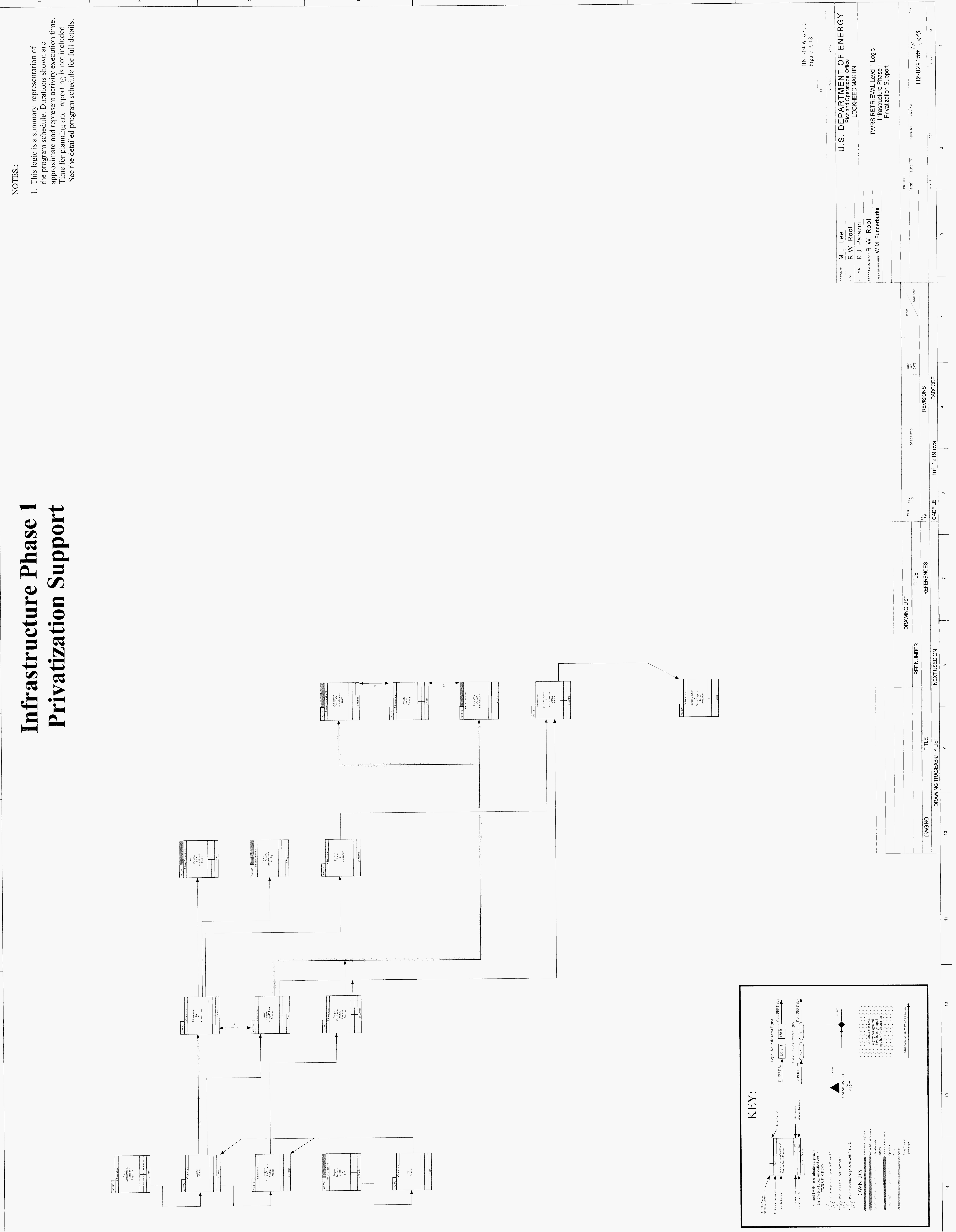




\section{DISTRIBUTION SHEET}

\begin{tabular}{|c|c|c|c|c|c|}
\hline \multirow{2}{*}{$\begin{array}{l}\text { To } \\
\text { Document Control Services }\end{array}$} & \multirow{2}{*}{$\begin{array}{l}\text { From } \\
\text { TWRS Confige }\end{array}$} & & & \multicolumn{2}{|c|}{ Page 1 of 1} \\
\hline & & tion Mana & ient & \multicolumn{2}{|c|}{ Date January 3, 1998} \\
\hline \multirow{2}{*}{\multicolumn{4}{|c|}{$\begin{array}{l}\text { Project Title/Work Order } \\
\text { Tank Waste Remediation Systems (HNF-1946) }\end{array}$}} & \multirow{2}{*}{\multicolumn{2}{|c|}{$\begin{array}{l}\text { EDT No. } 622746 \\
\text { ECN No. }\end{array}$}} \\
\hline & & & & & \\
\hline Name & MSIN & $\begin{array}{c}\text { Text } \\
\text { With Al? } \\
\text { Attach. }\end{array}$ & $\begin{array}{l}\text { Text } \\
\text { Only }\end{array}$ & $\begin{array}{l}\text { Attach./. } \\
\text { Appendix } \\
\text { Only }\end{array}$ & $\begin{array}{l}\text { EDT/ECN } \\
\text { Only }\end{array}$ \\
\hline
\end{tabular}

\section{J.N. Alibert}

M.P. Delozier

0. A. Halvorson

E.R. Hamm

J.O. Honeyman

K.N. Jordan

D.M. McDaniel

R.J. Murkowski

M.A. Payne

L.G. Peck

R.W. Powell

R.E. Raymond

S.H: Rifaey

B. Root

P.S. Schaus

S.E. Seeman

R.L. Treat

J.M. Vann

J.H. Wicks Jr

R.D. Wojtasek

$\begin{array}{ll}\text { S2-48 } & X \\ R 2-58 & X \\ G 3-21 & X \\ R 1-31 & X \\ \text { G3-21 } & X \\ \text { G3-21 } & X \\ H 7-06 & X \\ H 6-37 & 3 \text { copies } \\ \text { R2-58 } & X \\ H 6-35 & X \\ H 5-03 & X \\ \text { R2-38 } & X \\ \text { R1-56 } & X \\ \text { G3-21 } & 2 \text { copies } \\ \text { H5-03 } & X \\ \text { H6-35 } & X \\ \text { H5-03 } & 3 \text { copies } \\ \text { H6-37 } & X \\ \text { H7-07 } & X \\ \text { G3-21 } & 3 \text { copies }\end{array}$

B7-07. $x$

$x$

Central Files 